
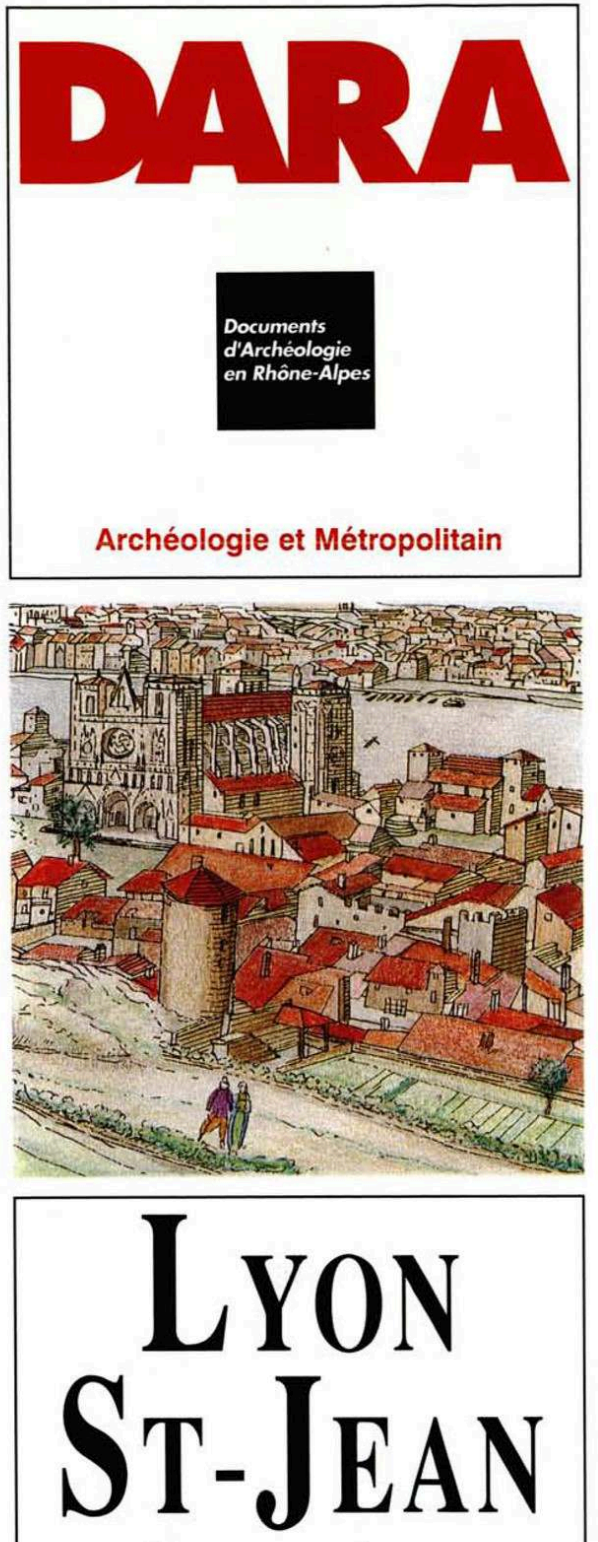

LES FOUILLES DE

L'ÎLOT TRAMASSAC

C. ARLAUD, J. BURNOUF, J.-P. BRAVARD, J.-M. LUROL, A. VÉROT-BOURRÉLY 


\section{Lyon Saint-Jean, les fouilles de l'îlot Tramassac}

Catherine Arlaud, Joëlle Burnouf, Jean-Paul Bravard, Jean-Marc Luriol et Agnès Vérot-Bourrély

DOI : $10.4000 /$ books.alpara. 2877

Éditeur : Alpara

Année d'édition : 1994

Date de mise en ligne : 3 juillet 2017

Collection : DARA

ISBN électronique : 9782916125299

\section{QboOks}

http://books.openedition.org

\section{Édition imprimée}

ISBN : 9782906190146

Nombre de pages : 150

Référence électronique

ARLAUD, Catherine ; et al. Lyon Saint-Jean, les fouilles de l'îlot Tramassac. Nouvelle édition [en ligne]. Lyon : Alpara, 1994 (généré le 12 janvier 2021). Disponible sur Internet : <http://books.openedition.org/ alpara/2877>. ISBN : 9782916125299. DOI : https://doi.org/10.4000/books.alpara.2877.

Ce document a été généré automatiquement le 12 janvier 2021.

(C) Alpara, 1994

Conditions d'utilisation:

http://www.openedition.org/6540 
La ville médiévale, héritière d'une longue histoire, a une mémoire topographique qui se signale dans le paysage par des repères monumentaux. Ainsi en est-il à Lyon de la cathédrale Saint-Jean. En revanche du quartier canonial constitué autour, il ne demeure que quelques fragments méconnus du mur d'enceinte. Ce volume contribue après les fouilles de l'avenue A. Max, et celles jouxtant la cathédrale, à faire découvrir l'évolution de ce secteur depuis le 1er siècle avant J.-C., quand la Saône coulait au pied de la colline de Fourvière, jusqu'à la mise en place, en un monde clos, d'un quartier réservé aux clercs au service de la première église du diocèse. Cette enquête, oeuvre d'archéologues, de géographes et d'historiens éclaire très concrètement, les modes de bâtir et de vivre des Lyonnais et contribue à résoudre certains points débattus de l'histoire de Lyon antique et médiévale. 


\section{SOMMAIRE}

\section{Propos liminaire \\ Élise Faure-Boucharlat}

\section{Introduction}

\section{Première partie. Fragments d'un paysage urbain}

PROBLÉMATIQUE ET HISTORIOGRAPHIE

QUAND LA SAÔNE COULAIT AU PIED DE LA COLLINE DE FOURVIÈRE

LE DÉVELOPPEMENT D'UN QUARTIER URBAIN

DEUX MONDES : DANS LES MURS ET HORS LES MURS

LE DÉMANTĖLEMENT DU QUARTIER CANONIAL

Deuxième partie. Étapes de l'urbanisation

TOPOGRAPHIE ET PREMIERS AMÉNAGEMENTS

DU Ier AU IIIe SIÈCLE

DU IVe AU VIe SIÈCLE

DU VIIe AU XIe SIÈCLE

DU XIIe AU XVIIIe SIÈCLE

L'ÉPOQUE CONTEMPORAINE

Troisième partie. Éléments de culture matérielle Jean-Michel Poisson et Bruno Claret

LA CÉRAMIQUE GALLO-ROMAINE

CÉRAMIQUE MÉDIÉVALE ET MODERNE

LA FAUNE

MONNAIES ET OBJETS MONÉTAIRES

Bibliographie 


\title{
Propos liminaire
}

\author{
Élise Faure-Boucharlat
}

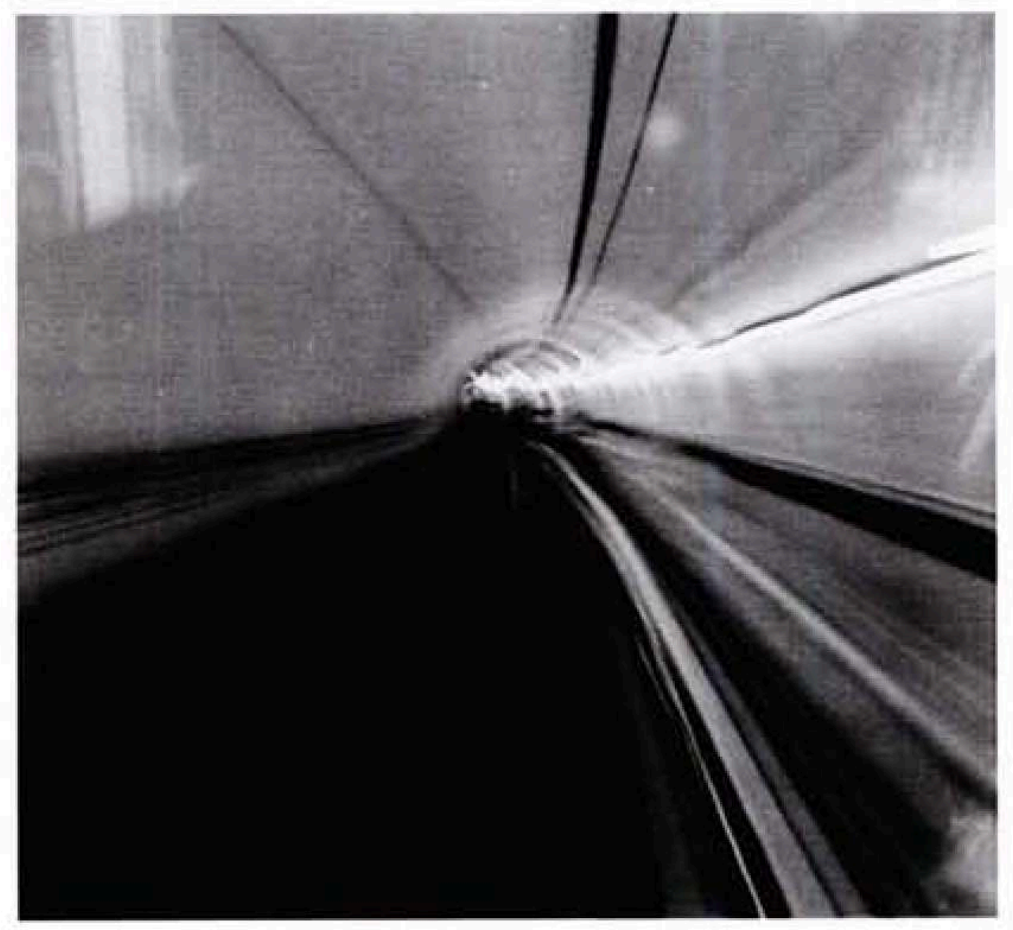

1 Avec ce volume des Documents d'Archéologie en Rhône-Alpes, le troisième de la série "archéologie et métropolitain", se déroule un nouvel épisode d'un long feuilleton que l'on pourrait appeler "de pièces et de morceaux", tant les fouilles menées à Lyon depuis plus de quinze ans livrent d'éléments de l'immense puzzle du paysage urbain.

2 Après les résultats des fouilles de l'avenue Adolphe Max en 1990 (D.A.R.A. n 3), ce n'est pas sans une certaine impatience que nous attendions la sortie de cet ouvrage consacré au site tout proche de TramassacCarriès. Eclairage complémentaire apporté à la genèse du quartier Saint-Jean, voici donc la chronique de deux années de travail durant lesquelles archéologues, historiens et géographes auscultèrent la trame d'un tissu 
urbain particulièrement chargé d'histoire, parce que stratégiquement placé : entre le relief de Fourvière et le cours de la Saône, à la limite de la ville et de ses faubourgs, à la frontière entre le quartier canonial et celui des habitats laïcs et, enfin, à la jonction entre l'Antiquité et le Moyen Age.

3 A qui s'étonnerait du délai qui sépare la fin de l'intervention de terrain, en 1986, de la publication des résultats, on opposera la surcharge qu'impose le rythme des transformations de la ville d'aujourd'hui aux archéologues lyonnais. En répondant toujours présents pour réaliser les fouilles de sauvetage qui permettront de lever les contraintes archéologiques pesant sur tel ou tel secteur, ces archéologues engrangent une documentation considérable. La digestion de cette documentation -son interprétation, la mise en forme des résultats pour leur restitution au public- est une tâche tout aussi considérable. Cependant, malgré leurs engagements dans d'autres opérations, les membres de l'équipe dirigée par Joëlle Burnouf et Catherine Arlaud ont mis leur point d'honneur à aller jusqu'au bout de la démarche. Mais, au fond, de ces difficultés, ne naît-il pas une certaine maturité de l'archéologie préventive qui, comme on en jugera ici, acquiert le recul nécessaire à une conception synthétique de l'évolution de la topographie urbaine. Maturité encore qui conduit à admettre, voire solliciter la remise en question comme une saine stimulation. Depuis les fouilles du quartier Saint-Jean, combien de sites explorés sur cette rive de la Saône ou dans la Presqu'île qui n'aient apporté leur lot d'informations nouvelles sur la topographie de Lyon, sur la chronologie du bâti, sur la vie quotidienne des anciens Lyonnais? Autant d'occasions d'apporter quelques retouches au manuscrit d'origine.

4 La sortie de l'ouvrage consacré à Tramassac intervient un peu plus de dix ans après la signature de la première convention entre le Ministère de la Culture et la SEMALY qui réglementait désormais les fouilles préalables aux travaux du métro. Compte tenu de son caractère alors exemplaire, l'événement connut un certain retentissement. $\mathrm{Ne}$ saurait-on mieux en marquer l'anniversaire qu'en éditant ces pages d'histoire lyonnaise que l'archéologie nous restitue fragment après fragment !

\section{AUTEUR}

\section{ÉLISE FAURE-BOUCHARLAT}

Conservateur du Patrimoine 


\section{Introduction}

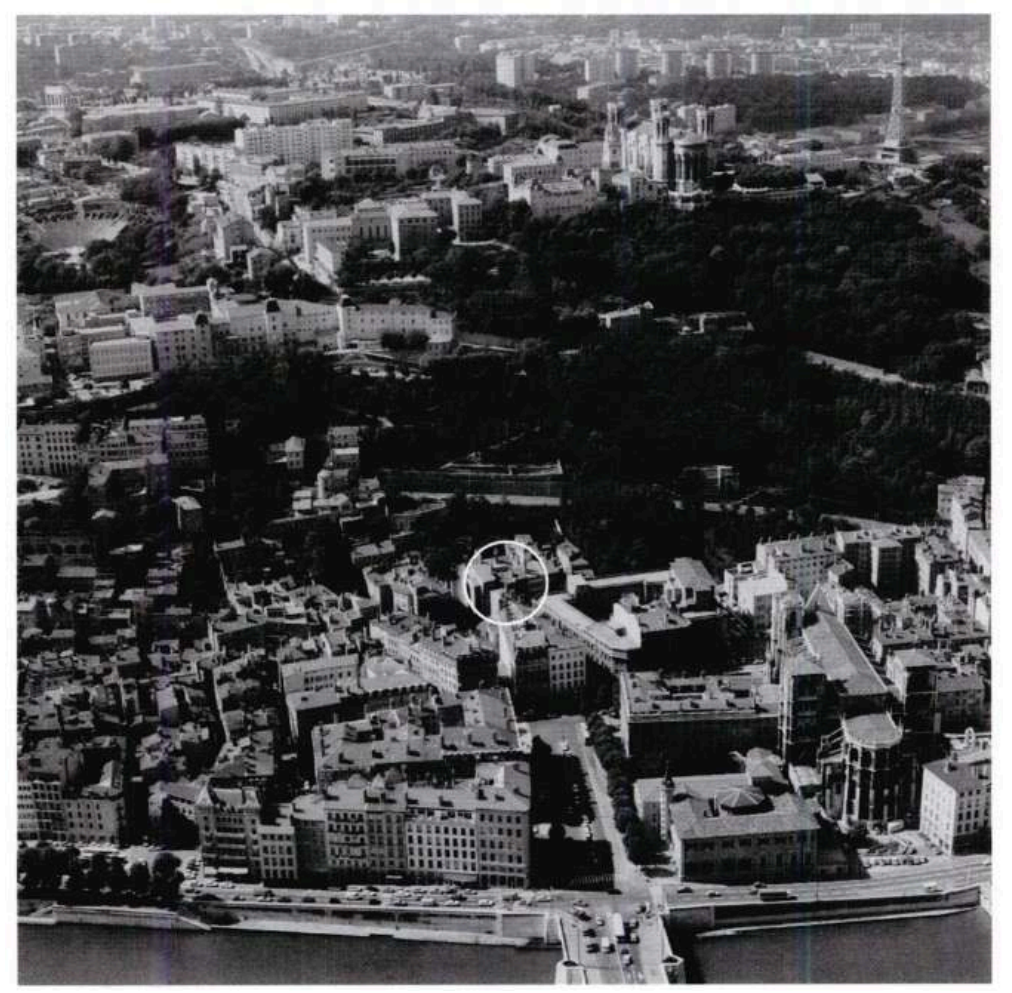

1 - VUE dU QUARTIER SAINT-JEAN AVEC L'îlot TRAMASSAC ET LA RUE JEAN CARRIÈs

(Cliché Semaly, J. Rutter)

1 Le chantier de l'îlot Tramassac-Carriès représente la troisième grande fouille archéologique de sauvetage ouverte dans le quartier Saint-Jean à Lyon, après celles de l'ensemble cathédral (Reynaud 1977) et de l'avenue Adolphe Max (Villedieu 1990).

2 La fouille de l'avenue Adolphe Max, liée au même programme de construction du métro que celle de l'îlot Tramassac, a déjà permis de préciser considérablement cette histoire, reconstituable jusqu'alors seulement par les textes et par des observations archéologiques demeurées ponctuelles, à l'exception de la fouille, encore inédite, de l'ensemble cathédral. 
3 L'îlot Tramassac se situe sur la rive droite de la Saône au pied de la colline de Fourvière (fig. 1, 2). Il est limité par la rue Mourguet au sud, la rue Tramassac à l'ouest, la rue Carriès au nord et la rue du Doyenné à Test. La quasi totalité des bâtiments de cet îlot a été reconstruite depuis la fin de l'Ancien Régime. L'installation des gares des funiculaires en particulier, a conduit au "curage" d'une bonne partie de sa surface. Bien que l'on se situe au coeur du Lyon médiéval, le problème de l'étude archéologique des bâtiments en élévations ne s'est pas posé, sinon pour l'immeuble situé à l'angle de la rue Jean Carriès, rescapé du réalignement de façade amorcé au XVIIe s., et qui est en cours de réhabilitation aujourd'hui.

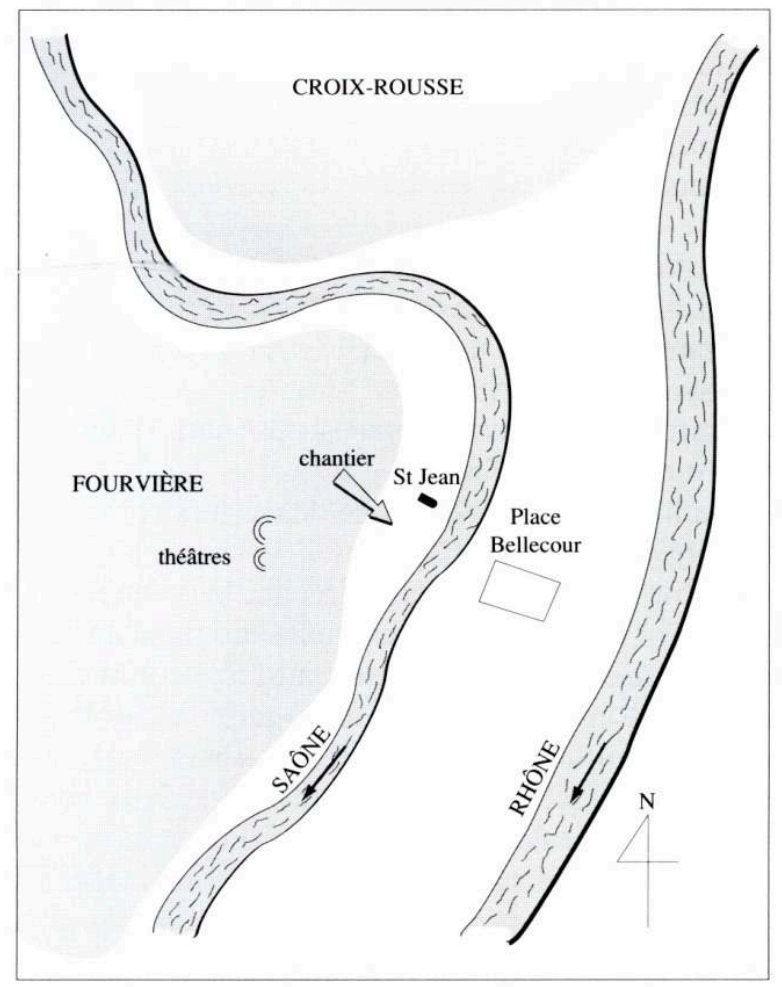

\section{2 - SITUATION DU CHANTIER DANS LYON}

Ce chantier pouvait-il apporter des données originales à la connaissance de l'évolution de la physionomie du quartier Saint-Jean et plus généralement, à l'histoire de Lyon ? La question devait être posée, la position de l'îlot étant en effet "stratégique" à plusieurs égards.

Il est d'abord situé à un emplacement où l'historiographie lyonnaise localise traditionnellement un ancien bras de la Saône, mis en évidence par A. Audin. La fouille devait donc permettre de préciser le tracé et la chronologie du comblement de la rivière et, partant de là, celle de la première urbanisation de l'Antiquité tardive. C'était aussi l'occasion d'étudier un secteur de la ville occupé pendant le Haut Moyen Age, période encore très peu documentée par l'archéologie.

6 Enfin, le fait que son emprise fut à cheval sur l'enceinte canoniale devait permettre d'étudier parallèlement l'évolution de l'habitat "noble" du chapitre, intra-muros, et celui d'un quartier laïc, plus modeste, adjacent, extra-muros.

7 A la suite des sondages exécutés en 1982 par le Service archéologique municipal de la ville de Lyon (Becker 1982), une convention signée par le Service régional de l'archéologie et le maître d'ouvrage, la SEMALY1 prévoyait des fouilles archéologiques à 
l'intérieur de l'îlot, emplacement de l'ancienne gare des funiculaires desservant les quartiers de Saint-Just et Fourvière. A cet endroit étaient en effet prévus la "descenderie" et l'échangeur de la ligne D du Métro. Les fouilles archéologiques ont été fractionnées à l'intérieur de cet îlot pour satisfaire aux exigences du chantier de génie civil et pour répondre aux contraintes de circulation du funiculaire. Le chantier de l'îlot Tramassac a été ouvert le 2 mai 1984 et s'est terminé en juin 1986 ; il a duré en tout 12 mois répartis sur deux ans. Le travail a été rendu difficile par son échelonnement, il y a eu pas moins de huit interventions, six sur l'emplacement du puits de la descenderie Saint-Jean, une sur une large tranchée occupant l'ensemble de la rue Jean Carriès et une sur un immeuble déclaré "en péril" à cause des travaux, toujours rue Jean Carriès (fig. 3). Ce morcellement de la fouille a compliqué à l'extrême, non seulement le travail de terrain, mais aussi le raccordement des données. La fouille de certaines zones a dû être répartie sur plusieurs interventions successives, de plus en plus profondes, comme Tramassac I et IV ou Tramassac II, IV et VI. Par ailleurs, un certain nombre de travaux connexes ont perturbé le terrain et l'ont amputé d'une surface importante.

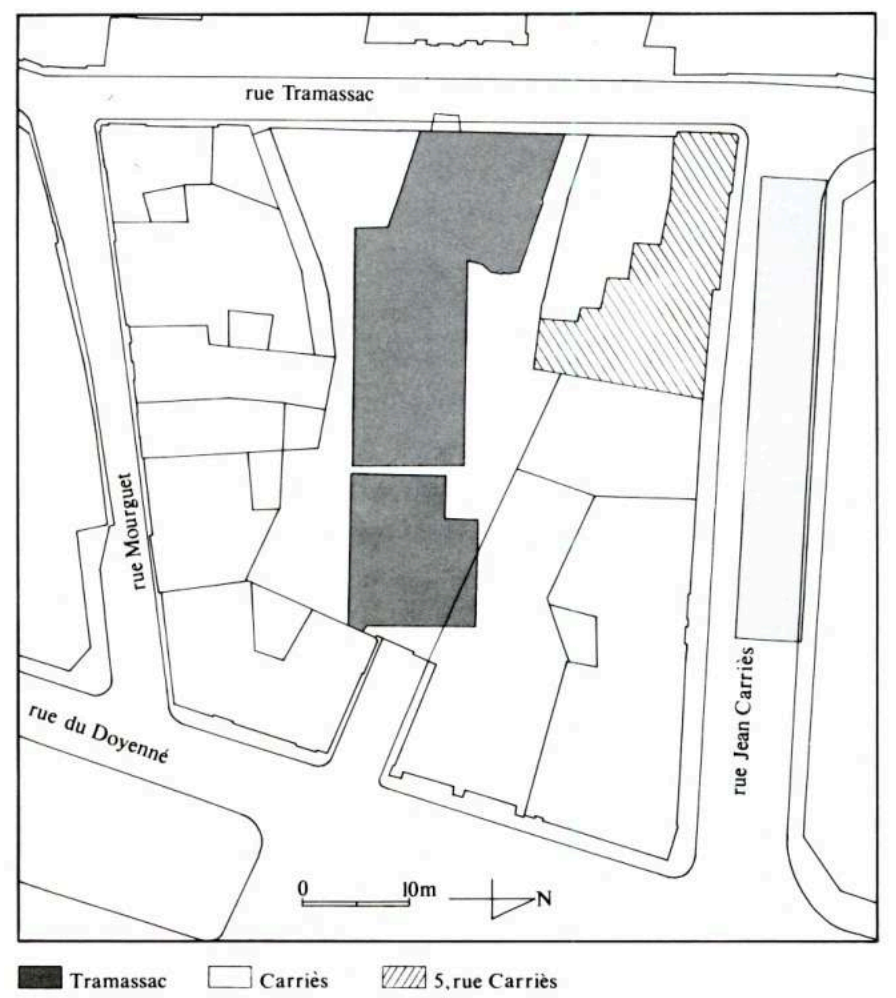

3 - EMPRISE DES DIFFÉRENTES FOUILLES DANS L'îLOT TRAMASSAC

Le calendrier des différentes interventions a été le suivant (fig. 4) :

- Tramassac I, de mai à septembre 1984, sur une surface de $100 \mathrm{~m}^{2}$;

- Rue Carriès, sauvetage urgent du 15 février au 4 avril 1985, sur une surface de $250 \mathrm{~m}^{2}$;

- Tramassac II, surveillance de travaux en juillet 1985 ;

- Tramassac III, du 15 juillet au 15 septembre 1985, sur une surface de $150 \mathrm{~m}^{2}$;

- Tramassac IV, janvier et février 1986, sur une surface de $150 \mathrm{~m}^{2}$;

- Tramassac V, du 15 avril au 20 juin 1986, sur une surface de $200 \mathrm{~m}^{2}$;

- Tramassac VI, surveillance de travaux entre septembre et novembre 1986 ;

$\cdot 5$, Rue Carriès, sauvetage urgent, une semaine. 


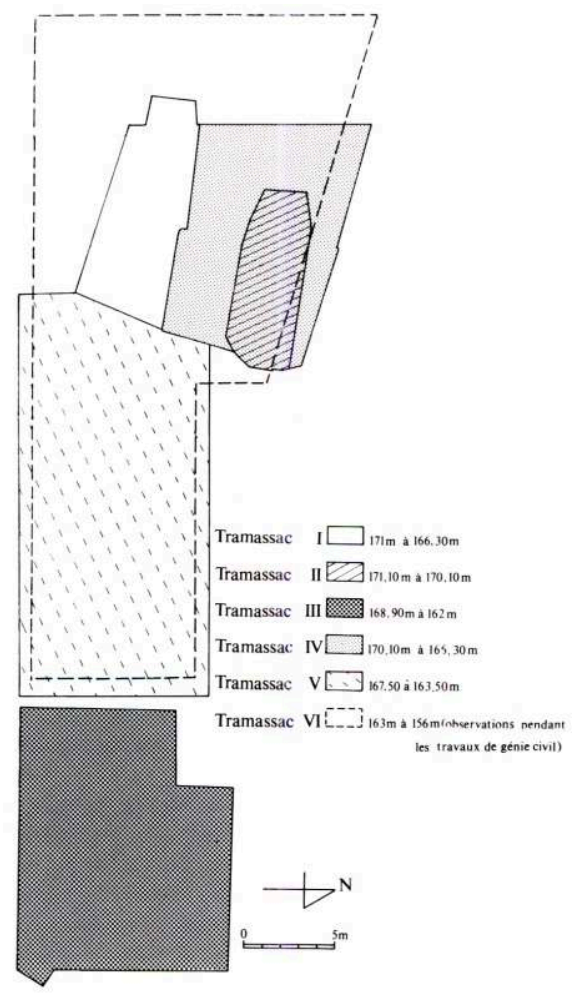

4 - LES INTERVENTIONS SUCCESSIVES SUR LE CHANTIER TRAMASSAC comprenait sept personnes, deux archéologues (C. Arlaud, J.-M. Lurol), une dessinatrice (C. Plantevin), une géomorphologue (A. Vérot-Bourrély), deux céramologues (G. Ayala et B. Macari-Poisson) et une archiviste (M.O. Mandy). On s'est efforcé d'impliquer, dans l'étude, des spécialistes des sciences environnementales, en particulier, la paléoostéologie, la dendrochronologie et la géomorphologie, afin de conduire une analyse des rapports de l'homme et de son milieu. Cette collaboration a donné lieu à diverses participations à des colloques, communications, tables rondes et séminaires

Le chantier de Tramassac correspond à une fouille urbaine sur un site à stratification complexe et continue, depuis l'état naturel initial antérieur à la première occupation (fin du Ier s. av. J.-C.) jusqu'à la dernière transformation à la fin du XIXe s. en gare du funiculaire, ce qui représente par endroits presque $10 \mathrm{~m}$ de puissance d'accumulation de sédiments archéologiques.

11 Les résultats sont présentés en trois parties :

- La première rassemble de manière synthétique les acquis de la fouille, interprétés et replacés dans le cadre général, géographique et historique, de Lyon, avec une attention particulière portée à la reconstitution de la topographie de la ville médiévale.

- La deuxième expose en détail la chronologie de l'occupation du sol sur l'emprise fouillée. Les résultats sont présentés par période (fig. 5). La "période" correspond à une étape homogène de la vie du site sur le plan culturel. Ce découpage en périodes, fondé sur des observations archéologiques, est donc indépendant des schémas historiques traditionnels.

- Enfin, la troisième partie rassemble des études spécialisées dont l'originalité et la richesse de leurs conclusions méritaient une présentation particulière. La sélection de ces contributions a été faite en fonction de deux critères, d'une part l'apport original de l'étude dans son ensemble - même si les résultats ont été utilisés et intégrés dans le corps du texte - et d'autre 
part le caractère inédit des résultats. Les études ou parties d'études ayant déjà fait l'objet de publications ont été écartées afin de ne pas alourdir l'ouvrage par des redites. Les études strictement techniques ou les simples identifications ont été directement intégrées dans l'exposé des résultats.

Il est important de préciser un trait original de ce gisement: eu égard au volume fouillé, la quantité du mobilier archéologique est peu importante. En particulier, on observe l'absence totale de mobilier métallique (toutes catégories confondues) et la faible représentation du mobilier céramique complet pour certaines périodes. Les seuls endroits où des lots ont pu être mis au jour sont les milieux clos (dépotoirs). Ils ont été traités comme le requiert ce mode de gisement. Le mobilier céramique a permis d'établir une chronologie fine, en particulier pour les périodes encore mal connues à Lyon de l'Antiquité tardive et du Haut Moyen Age. Le feuilletage des couches archéologiques, leur fouille fine et l'enregistrement rigoureux ont permis de faire l'étude détaillée de ces lots et de repérer des traceurs encore inconnus jusque-là.

\begin{tabular}{|c|c|c|}
\hline Périodes & Dates & Evénements \\
\hline 1 & Ier s. av. J.-C. - lère moitié du Ier s. ap. J.-C & La topographie avant l'occupation humaine \\
\hline 2 & 2ème moitié du Ier s. ap. J.-C. - fin IIIe s. & Le remplissage du bras de Saône \\
\hline 3 & fin IIIe s. - début IVe s. & Création d'une voie nord-sud \\
\hline 4 & fin IVe s. - début Ve s. & Construction d'un ensemble monumental \\
\hline 5 & VIe s. & Un habitat \\
\hline 6 & fin VIe s. & Abandon partiel du site \\
\hline 7 & fin VIe $\mathrm{s}$. & Vestiges d'un bâtiment \\
\hline 8 et 9 & fin VIe - début VIIe s. & Un habitat mérovingien \\
\hline 10 & fin VIIe - début VIIIle s. & $\begin{array}{l}\text { Un habitat dans la zone ouest et un abandon } \\
\text { de la zone est }\end{array}$ \\
\hline 11 & IXe - Xe s. & Un habitat carolingien \\
\hline 12 & IXe - Xe s. & Réaménagement de cet habitat \\
\hline 13 & XI es. & Une nouvelle structure d'habitat \\
\hline 14 & XIIe s. & $\begin{array}{l}\text { Edification du mur d'enceinte et d'une maison } \\
\text { canoniale ; organisation du parcellaire } \\
\text { rue Tramassac extra-muros }\end{array}$ \\
\hline 15 & XIIIle - XVes. & $\begin{array}{l}\text { Exhaussement des niveaux de sol et } \\
\text { construction rue Tramassac; } \\
\text { la maison du doyen intra-muros }\end{array}$ \\
\hline 16 & XVIe s. & $\begin{array}{l}\text { Quelques modifications dans le bâti de } \\
\text { la rue Tramassac ; l'hôtel du Doyenné }\end{array}$ \\
\hline 17 & XVIIIe s. & id..... \\
\hline 18 & XVIIIle s. & id..... \\
\hline 19 & XIXe s. & $\begin{array}{l}\text { L'hôtel du Doyenné vendu comme Bien Nationa } \\
\text { restructuration du quartier }\end{array}$ \\
\hline 20 & fin XIXe s. & $\begin{array}{l}\text { Démolition de l'intérieur de l'îlot } \\
\text { et construction du "funiculaire" }\end{array}$ \\
\hline
\end{tabular}

5 - LES PÉRIOdES MISES EN ÉVIDENCE PAR L'ARCHÉologie

\section{NOTES}

1. SEMALY : Société d'Economie Mixte du Métropolitain de l'Agglomération Lyonnaise. 


\section{Première partie. Fragments d'un paysage urbain}

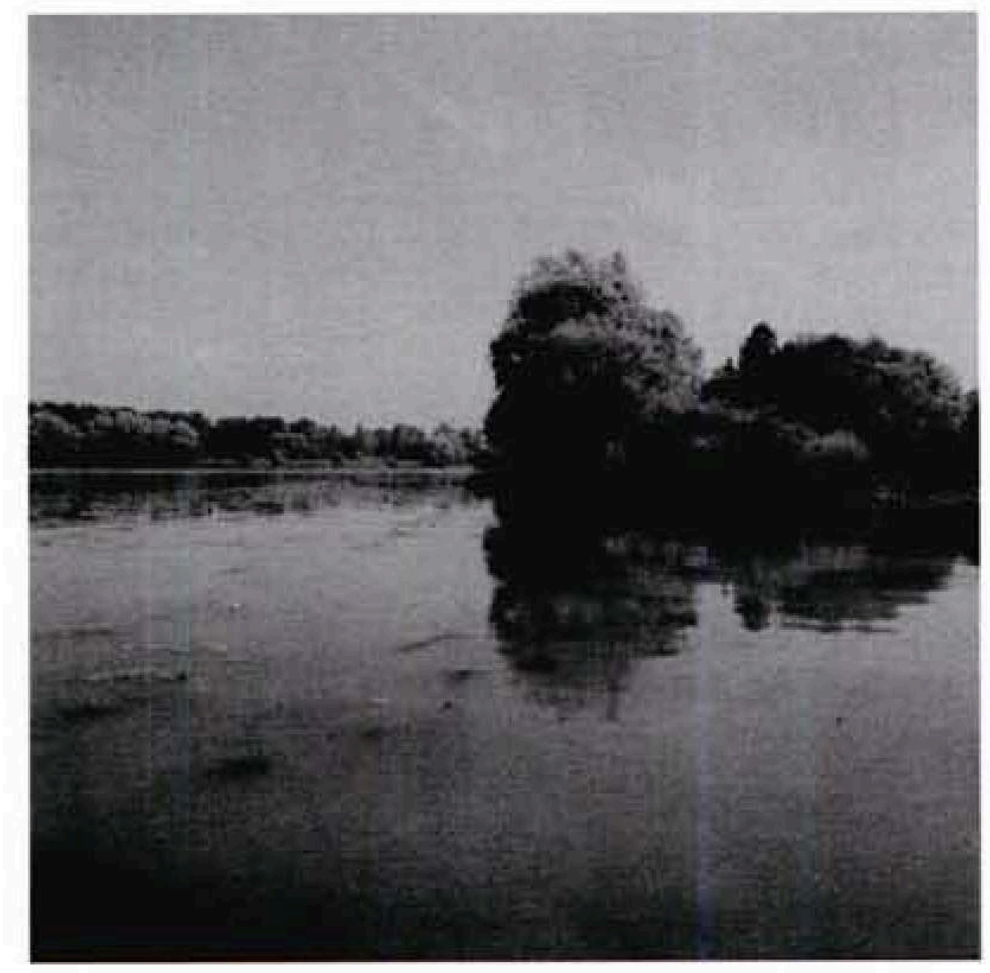

1 Le géographe moderne Vidal de la Blache écrivait: "en déposant latéralement alluvions, en formant une série d'îles leurs destinées à se souder, les deux fleuves, le Rhône et la Saône, ont circonscrit un berceau de ville". Il voulait montrer que le site de Lyon bénéficiait autant de ce double fossé dessiné par les deux fleuves que de l'acropole de Fourvière vantée par Strabon, son antique prédécesseur. Ces deux visions reflètent une tendance générale de l'évolution de la topographie urbaine de Lyon.

2 Nous intéresse ici le centre médiéval, coincé entre les pentes escarpées de Fourvière et les berges de la Saône, et dominé par la silhouette imposante de la cathédrale SaintJean. L'archéologie montre les vicissitudes de son développement, dans une zone 
encore inhospitalière au début de notre ère, le long d'une rivière au cours encore très fluctuant, qui n'est rejetée que progressivement à son emplacement actuel. D'abord lieu d'installations dispersées sur les rives du bras de la rivière, le quartier n'est réellement urbanisé qu'au IIIe s. Il devient le centre de la ville dès le IVe s., alors que la cité du Haut Empire, au sommet de Fourvière, périclite et que ses édifices publics sont transformés en carrières de pierre. Une des raisons de cette expansion est peut-être la volonté des autorités ecclésiastiques d'élire un nouveau lieu pour l'établissement des édifices du culte chrétien. L'originalité de l'urbanisation de la rive droite de la Saône entre l'Antiquité tardive et le XIIIe s. vient en effet de ce que, contrairement à d'autres villes comme Metz ou Strasbourg, le groupe cathédral du IVe s. n'a pas choisi de s'installer sur le site de l'ancienne civitas, dans le palatium ou le castrum antique, mais sur un site, non pas vierge, mais peu ou pas urbanisé, au moment où l'espace disponible devient plus vaste par le rattachement de l'ancienne île Saint-Jean à la terre ferme.

3 La prééminence du quartier Saint-Jean se prolonge et se fortifie tout au long du Moyen Age, alors que les archevêques, qui consolident aussi leur pouvoir temporel, s'entourent d'une véritable cité ecclésiastique. La puissance du pouvoir archiépiscopal s'affiche par la reconstruction de la cathédrale à partir du début du XIIe s. et, au cours du même siècle, par l'édification du mur de l'enceinte clôturant le quartier canonial, qui devient une véritable ville dans la ville.

4 A partir de la fin du Moyen Age, le pouvoir ecclésiastique n'est plus, loin s'en faut, le principal moteur du développement de la ville qui, comme beaucoup d'autres dans l'Occident médiéval, connaît une croissance polycentrique. L'évolution de la physionomie du quartier est alors largement soumise à la multiplication des initiatives individuelles. L'enceinte est disloquée au gré des mutations de propriétés et des reconstructions, les spécificités de la cité ecclésiastique s'estompent progressivement. Les aménagements urbains affectent de nouveaux quartiers, surtout dans la Presqu'île. Au XVIIIe s., l'habitat de Saint-Jean est déjà fortement délabré et sans doute dans un état assez proche de celui que l'on pouvait encore observer au milieu du XXe s., malgré quelques reconstructions ponctuelles.

\section{PROBLÉMATIQUE ET HISTORIOGRAPHIE}

Ce quartier de Lyon a déjà fait l'objet de nombreuses observations archéologiques comme le montre l'inventaire dressé par F. Villedieu (1990, p. 139-144). On retiendra en particulier quelques opérations importantes: celle du groupe cathédral (Reynaud 1977), plus au sud celle de la rue de la Quarantaine (Jacquin 1981), et de Saint-Laurentde-Choulans (Reynaud 1985) et enfin, celle de l'avenue Adolphe Max (Villedieu 1990). Il convient d'ajouter la fouille récente (1992) de la place Benoît Crépu, menée par V. Monnoyeur, qui a apporté de nouvelles données sur l'occupation de l'Antiquité tardive, ainsi que l'étude de la Maison de la Chamarerie (Mounier 1986, Arlaud-Rolland 1990).

6 Pour ce qui est de la topographie ancienne de ce secteur et du tracé suivi par la Saône durant l'époque antique, A. Audin se risque à une hypothèse au vu d'observations archéologiques: "D'importants travaux entrepris en 1957 pour la construction de l'immeuble $\mathrm{n}^{\circ} 3$ rue Tramassac ont révélé l'existence d'un bras d'eau qui longeait à l'orient le tracé des actuelles rues du Boeuf et Tramassac. Ce bras, c'est l'évidence, se détachait de la Saône au fond du golfe du Change pour la rejoindre à mi-chemin entre Saint-Jean et Saint-Georges, là où le frère Benoît a vu, à l'occasion des travaux, une 
énorme masse de remblais..." (Audin 1959). Ainsi, ces sondages ont conduit A. Audin à situer le passage d'un bras de la Saône directement au pied de la colline, délimitant à l'est une île que nous appelons l'île Saint-Jean (fig. 6).

7 Les fouilles ont donné l'occasion de multiplier les observations sur la structure d'une partie de l'île et sur le remplissage alluvial de cet ancien chenal, dont l'existence est confirmée, au moins pour la fin de l'Antiquité. Il est en effet possible de préciser la géométrie du chenal originel de la Saône elle-même et les étapes de son comblement progressif par les eaux et par les hommes à l'époque antique.

L'étymologie du mot "Tramassac" a suscité beaucoup d'interprétations, parfois franchement fantaisistes. Les noms anciens sont : Retro-Marsal, Tres Marsas, Trans Marsas, Tres Marsal. Certains ont voulu lire Retro marsam, soit "derrière la masse du temple dédié à Mars", d'autres avec Tres Marsas, ont lu "Trois Massacre", etc. Dans un dictionnaire de vieux français (Niemeyer s. d.) deux mots ont retenu notre attention, mara (masra, marage) qui signifie marais, marécages et mansus (massa, massage) qui signifie soit l'ensemble des terres équivalant à un manse, soit une demeure, soit enfin un domaine.

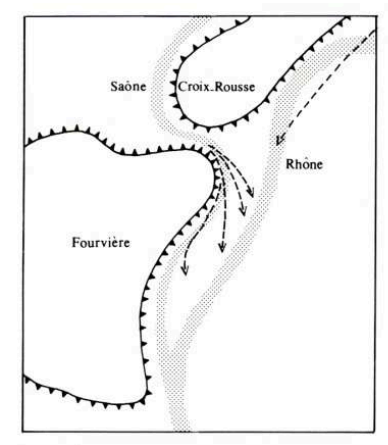

1. Holocène indetermine

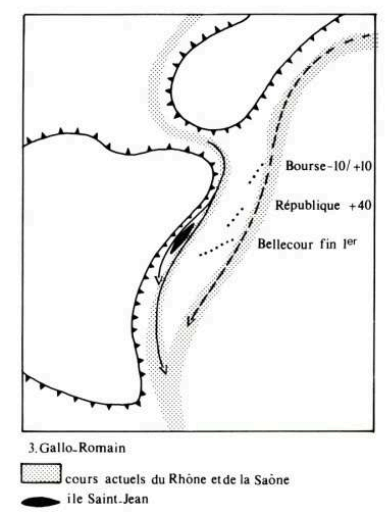

6 - schémA de LA Mise en PLACE dU SITE
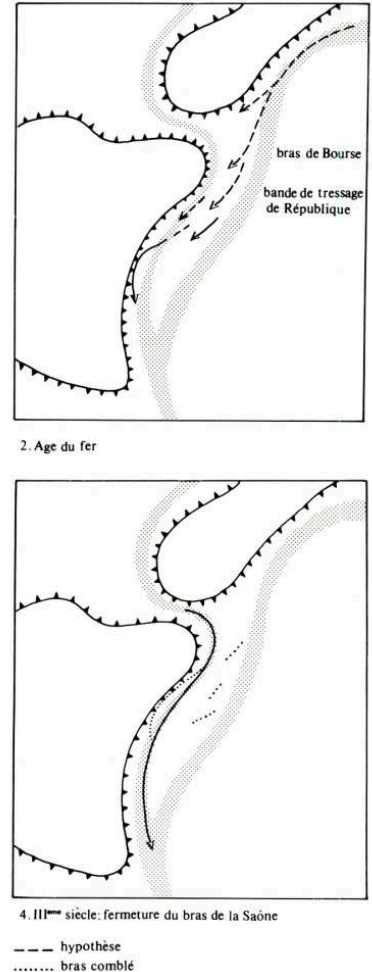

9 L'installation, au Haut Moyen Age, du groupe épiscopal (Reynaud 1977) sur la rive droite de la Saône actuelle, est une preuve du dynamisme du quartier à cette époque. Dans la région lyonnaise, ce sont les fouilles des édifices religieux et des nécropoles (Reynaud 1981 et 1986), croisées avec une relecture des textes anciens, qui ont fait progresser les connaissances. Lyon a eu des évêques d'envergure, personnalités autant politiques que religieuses, qui ont donné à la ville un rayonnement dépassant largement la région. Ce rayonnement se traduit dans le paysage urbain par une architecture religieuse de prestige. Comme ils sont mis en œuvre par des 
ecclésiastiques, les textes ne fournissent en revanche aucun détail sur l'habitat civil associé, que seule l'archéologie permet d'appréhender.

10 La ville connait, au cours des VIIe et Ville s., une instabilité politique et religieuse (Février 1986). Toutefois, l'Eglise de Lyon reste le principal pouvoir. La source écrite la plus précise concernant cette époque est le Bref de Leidrade (813). Evêque de Lyon à la fin du Ville s. et au début du IXe s., Leidrade est mandaté par Charlemagne afin de restructurer le diocèse. De fait, il fut fort entreprenant, puisque ce texte énumère une longue liste de constructions et reconstructions d'églises.

11 A partir du XIIe s., la documentation écrite plus abondante permet de se faire une meilleure idée de l'occupation de la rive droite de la Saône. Elle reste à dominante ecclésiastique ; les chanoines, comtes de Lyon, détiennent tous les pouvoirs. Cet état de fait continuera après l'obtention d'une charte d'affranchissement de la ville en 1320 . L'enceinte canoniale ${ }^{2}$ de Saint-Jean, construite au XIIe s., est le reflet de cette puissance; elle enferme le groupe cathédral et tous les bâtiments liés à la vie d'une communauté d'ecclésiastiques. Le tracé de la muraille est connu par les textes, les représentations graphiques, quelques vestiges encore en élévation, et les fouilles archéologiques (Reynaud 1977 et 1981). La partie sud-ouest (c'est-à-dire l'emprise des zones de fouilles de Tramassac-Carriès) n'avait pu être observée, aussi le tracé donné par certains auteurs (Sachet 1914) restait-il hypothétique.

12 C'est dans le cadre de cette problématique très diversifiée qu'est intervenue la fouille. Son emprise permettait à l'évidence de saisir l'évolution des berges de la Saône et de leur occupation, de vérifier le tracé de la muraille, ainsi que l'effet de cette "frontière" sur l'évolution de l'habitat extra et intra-muros.

\section{QUAND LA SAÔNE COULAIT AU PIED DE LA COLLINE DE FOURVIÈRE}

13 C'est de manière récente que les contraintes de l'environnement sont prises en compte dans les interprétations archéologiques. En milieu fluvial, les interactions hommenature sont particulièrement complexes à analyser du fait de la mobilité spatiale des cours d'eau et de leur activité fluctuante. Les réponses humaines à cette instabilité, comme les réactions des organismes fluviaux aux interventions anthropiques, sont aléatoires et subissent un décalage temporel fréquent. Cette approche doit conduire les historiens à interpréter les lieux d'occupation d'une manière moins mécaniste que cela a pu être fait dans le passé (Bravard et alii 1989).

14 A Lyon, le développement de la ville présente des caractères différents selon les quartiers, et l'appropriation par l'homme de ce milieu ingrat est l'objet d'une histoire complexe, depuis la protohistoire ancienne (Vaise) jusqu'à la "chenalisation" du Rhône au XIXe s.

15 L'étude géomorphologique du quartier Saint-Jean, menée lors des fouilles archéologiques de l'avenue A. Max, de la rue Carriès et de l'îlot Tramassac, a permis de reconstituer le paléoenvironnement du site et de confirmer l'hypothèse avancée par Ph. Russo et A. Audin (Russo et alii 1961). Il s'avère en effet que la Saône coulait au pied de la colline de Fourvière au Ier s. av. J.-C. (fig. 6).

16 Le témoignage, encore partiel, de l'archéologie ne fait pas remonter l'installation humaine dans la plaine alluviale du Rhône à une époque antérieure au Ier s. avant J.-C.. 
L'expansion de l'habitat gallo-romain dans la plaine pourrait au demeurant s'inscrire dans la logique simple d'une croissance urbaine à la recherche d'espaces plans faciles à urbaniser.

17 Le sous-sol de la plaine lyonnaise est loin d'avoir livré tous ses secrets, mais l'absence de vestiges plus anciens, remontant au Néolithique ou à l'Age du Bronze, ne laisse pas de surprendre tant leur présence est généralisée dans la plaine de la Saône. L'idée vient alors que cette plaine, aire de confluence mythifiée par l'Histoire, est à peine plus ancienne que l'installation antique : il est très probable qu'au premier Age du Per un Rhône puissant, chargé d'alluvions caillouteuses, et soumis à des crues brutales, a occupé l'étendue de la plaine alluviale; il a exhaussé son lit, alluvionné les sites actuels de la presqu'île et de Saint-Jean et repoussé la Saône contre le coteau de Fourvière. Jamais, sans doute, le paysage de "brotteaux" n'a dû connaitre pareille extension, mêlant les îles caillouteuses brièvement exhaussées par les limons de débordement aux bras courants et aux lônes dans un paysage de vastes saulaies couchées par les crues. Les troncs d'arbres extraits des alluvions datent cette phase de paroxysme qui a oblitéré toute trace d'ancienne occupation.

Sur plusieurs grands cours d'eau d'Europe, une phase de rémission est ensuite attestée et correspond chronologiquement aux premiers siècles de l'expansion romaine : les crues diminuent de fréquence et d'intensité, les rivières creusent leur lit et simplifient leur tracé. C'est dans un environnement plus amène que l'homme a pu conquérir la plaine et bâtir les cités fluviales de la vallée du Rhône (Macé et alii 1991).

Le confluent du Rhône et de la Saône, "berceau" de la ville, serait ainsi un état transitoire de la vie des deux fleuves, saisi par les hommes aux premiers siècles de notre ère et maintenu face aux vicissitudes hydrologiques du Moyen Age et de l'époque moderne.

20 Sur la rive droite de la Saône, qui coulait au début de l'ère au pied de la colline de Fourvière, un appontement fut découvert lors de la fouille. Il permettait l'usage de l'étroite bande de terre localisée au contact de la berge et du versant (Archeolabs a fourni les dates dendrochronologiques de 27 av. J.-C., 8 av. J.-C., 18 ap. J.-C.). Parallèlement, un second bras de la Saône se met en place à $150 \mathrm{~m}$ plus à l'est. Peu après le milieu du Ile s. ap. J-C., un habitat gallo-romain s'installe dans la partie ouest de l'île que délimitent ces deux bras (Villedieu 1990). Dans le courant de la première moitié du IIIe s., le bras de Saône entre l'île Saint-Jean et la colline est comblé progressivement et l'espace ainsi dégagé est colonisé par l'homme (rue Carriès), en même temps que l'est de l'île Saint-Jean (Villedieu 1990). Les derniers micro-chenaux actifs en temps de crue fonctionnèrent jusqu'à la fin du IIIe s. A partir de l'Antiquité tardive (extrême fin du IIIe s.-début du IVe s.), on peut raisonnablement considérer que la surface utilisable pour l'habitat sur la rive droite de la Saône est en place; ce secteur devient donc urbanisable. L'intervention humaine a désormais suffisamment stabilisé le cours de la rivière pour qu'il se maintienne au même emplacement jusqu'à nos jours.

\section{LE DÉVELOPPEMENT D'UN QUARTIER URBAIN}

21 Contrairement à l'habitude, le chantier de Tramassac a livré une épaisse sédimentation archéologique pour cette période charnière du premier Moyen Age. Elle permet de mieux appréhender la mise en place de l'habitat à la périphérie du groupe cathédral, au pied de l'escarpement de Fourvière. L'étude de la succession des aménagements et du 
mobilier associé permet de proposer une périodisation culturelle fondée sur la qualité du bâti qui fait apparaître deux phases, la première s'achève au VIe s., la seconde au XIe s.

- De la fin du IIIe s. au début du VIe s., est mis en place au pied de la colline un grand axe de circulation, dont le tracé est repris par la rue Tramassac (nous emploierons les noms actuels des rues afin d'éviter toute confusion). De vastes bâtiments lui sont associés, qu'il faut vraisemblablement rattacher au groupe épiscopal, la domus ecclesiae, mentionnée par Grégoire de Tours, en raison de la ressemblance des modes de construction. Leur utilisation s'arrête au XIe s.; l'ensemble du terrain continue à être occupé, mais sans "grande architecture". La documentation écrite nous apprend que l'évêque Leidrade (Coville 1928) entreprend la reconstruction des bâtiments de la domus ecclesiae, sans indication d'emplacement. Ce n'est vraiment qu'avec la construction du palais épiscopal par l'archevêque Humbert (1070-1076) que la localisation de la résidence de l'évêque sur le bord de Saône est certaine. Il en reste d'ailleurs des vestiges dans le palais épiscopal actuel (Guigue 1867).

Il est très tentant de voir dans les constructions découvertes à Tramassac des bâtiments du premier complexe épiscopal. On observe que l'espace fouillé, occupé pendant tout le Haut Moyen Age, reste une grande parcelle jusqu'au début du XIIe s., lorsqu'est construite l'enceinte canoniale. C'est à l'est de ces terrains qu'est bâti l'hôtel du doyen qui succède à cet emplacement à la maison de l'évêque Aulam veteram reedificavit (Guigue 1867). Ce qui nous incite à penser que le premier palais épiscopal était plutôt au pied de la falaise de Fourvière que sur les bords de la Saône. L'espace resté libre au coeur de l'îlot dépendait du doyen jusqu'à la fin du Moyen Age

Nous proposons donc le schéma d'évolution suivant:

- au Ve s. est construit un premier grand bâtiment situé le long de la voie ; à la fin du VIe s., lui succède un autre grand bâtiment de même nature, mais situé plus à l'est, qui est utilisé jusqu'au IXe s. La qualité du bâti permet de proposer, en relation avec la morphologie du parcellaire postérieur et sa dévolution juridique, que ces constructions successives pourraient appartenir aux différents états des palais épiscopaux du premier Moyen Age (première résidence de l'évêque).

-à l'ouest de l'espace, du VIIe à la fin du XIe s., de nouvelles constructions, utilisant la pierre et la terre, se succèdent au même emplacement. Six états du bâti ont été étudiés pour une épaisseur de sédimentation d'un mètre. La découverte de ces niveaux du Haut Moyen Age prouve que l'occupation du site ne connaît pas de hiatus.

L'étude des niveaux d'occupation de Tramassac est donc fondamentale pour la connaissance de l'habitat urbain de cette époque qui reste encore limitée, comme le souligne M. Fixot: "des formes des maisons urbaines nous ignorons à peu près tout, sinon qu'il en existait à étage, ce que signale Grégoire de Tours à Angers où l'une d'elles, qui était une demeure aristocratique, comptait au moins trois niveaux. Dans les zones suburbaines, les formes des habitats ont pu être encore plus diverses qu'elles ne l'étaient à l'intérieur des remparts, depuis la simple cabane de type rural jusqu'à la domus suburbaine" (Fixot 1980, p. 523-524). La fouille a montré que des structures antiques continuent à être utilisées, tandis que des bâtiments nouveaux sont construits selon d'autres techniques propres au Haut Moyen Age. Surtout, elle a permis d'individualiser des unités d'habitation. Celles-ci ressemblent à la "maison élémentaire" dont J. Chapelot et R. Fossier donnent la définition (Chapelot 1980, p. 222), bien que leur étude porte sur la maison rurale. Cette maison comporte une seule pièce 
contenant un emplacement pour le feu et semble être une forme classique entre le Ve et le XIe s., bien qu'on puisse en retrouver des survivances à des dates plus récentes. Toutefois, les structures mises au jour dans l'îlot Tramassac ne reproduisent pas le schéma désormais bien connu en milieu rural pour ces périodes, celui de maisons excavées. Ici les fondations et les matériaux des couches de démolition permettent d'imaginer des élévations en pierre ou en terre. Quant à la toiture, aucun indice ne permet d'émettre d'hypothèse sur sa nature. D'autres fouilles, réalisées à Lyon depuis, ont permis de corroborer les observations faites sur le site de Tramassac ${ }^{3}$.

Les structures d'habitat du Haut Moyen Age conservées diffèrent totalement des constructions gallo-romaines et ne répondent, semble-t-il, à aucun "schéma d'urbanisme" (tel qu'on peut le concevoir aux périodes antérieures ou postérieures). Si des bâtiments antiques sont conservés, les nouvelles implantations s'effectuent sans volonté de respecter les anciens alignements. Hasard? Exploration trop limitée? Certes, d'autres fouilles archéologiques sont nécessaires pour infirmer ou vérifier si les caractéristiques de ce site peuvent être étendues à son environnement, mais ici la négation des traditions romaines est évidente à partir du VIe s.; la réutilisation de structures gallo-romaines est le seul prolongement qui existe, sans réelle volonté non plus de les sauvegarder. Cette réutilisation est aussi notée par F. Villedieu pour les fouilles de l'avenue A. Max (située à proximité immédiate de l'îlot Tramassac) à partir du VIIe s. Ce constat est fait sur nombre d'autres sites, mais quelquefois moins tardifs, comme à Poitiers, où les fouilles de l'ancien évêché ont montré "un recul des traditions romaines dès le IIIe s." (Le Masne de Clermont 1987, p. 162). A Fréjus, P.-A. Février note "que les continuités urbaines ne s'observent que en ce qui concerne le cadre monumental ou ses dépendances" (Février 1986). M. Fixot souligne avec un "contreexemple" cet état de fait constant lors des fouilles du palais épiscopal d'Aix-enProvence. “...c'est l'indice archéologique, rare pour les régions méridionales, d'une conservation du tissu urbain antique, ou au moins de certaines parties de celui-ci" (Fixot 1988).

\section{DEUX MONDES : DANS LES MURS ET HORS LES MURS}

Deux modes d'occupation du sol, intra et extra muros, vont coexister : celui du quartier canonial à l'est du mur d'enceinte et celui du quartier laïc à l'ouest.

\section{DONNÉES HISTORIQUES}

28 Au XIIe s., le paysage politique se complique. Le pouvoir impérial est très théorique. La noblesse locale est peu encline à une alliance germanique. L'empereur s'efforce de pallier ces problèmes en essayant d'entretenir un équilibre entre les puissances locales et en jouant sur le caractère provisoire de la puissance de l'archevêque. Cette politique passive aboutit à un échec entériné par la Bulle d'Or de 1157. Dans ce texte, Frédéric Barberousse fait concession à l'archevêque "du corps entier de la cité de Lyon et de tous les droits régaliens établis dans l'archidiocèse à l'est de la Saône, à l'intérieur et à l'extérieur de cette ville". 
et ils entrent en conflit avec le pouvoir de l'archevêque dont la principauté s'étend aussi sur cette région. D'autre part, les comtes de Forez briguent le comté de Lyon et n'hésitent pas à recourir à l'engagement armé contre les archevêques. La bataille d'Yzeron en 1158, gagnée sur le terrain par les comtes de Forez, est déjà perdue politiquement pour eux en raison de l'appui apporté à l'archevêque par l'empereur Frédéric Barberousse. La complexité de la situation politique se traduit par le flou juridique délibéré du texte de la Bulle d'Or.

30 L'accord n'est définitif qu'avec le traité de Montbrison en 1173, où l'Eglise de Lyon renonce à ses biens dans le bassin de la Loire, tandis que le comte de Forez lui cède le titre de "comte de Lyon" et tous les droits sur le Lyonnais. Prince d'Empire, l'archevêque possède tous les droits régaliens ; il est alors quasi indépendant.

31 Jusqu'en 1312, la prospérité provoque le développement d'une bourgeoisie marchande à Lyon. Les conflits entre les pouvoirs économiques et politiques conduisent cette nouvelle classe sociale à se rebeller avec violence contre le carcan épiscopal pour conquérir son indépendance.

Les premiers accords entre la communauté des bourgeois et les archevêques interviennent entre 1193 et 1206. Malgré cela, plusieurs insurrections éclatent au XIIIe s. contre le pouvoir de l'archevêque. En 1269, la révolte est tellement violente qu'elle oblige les chanoines à quitter le quartier canonial et à se replier sur la colline de SaintJust.

33 Le XIVe s. marque un tournant décisif dans les rapports entre la communauté urbaine et son suzerain par l'intervention et la montée de pouvoirs nouveaux. Le roi de France, profitant d'une nouvelle insurrection en 1310, prend possession de la ville en 1312 par le traité de Vienne. La ville de Lyon acquiert enfin son autonomie administrative en 1320.

34 En définitive, au terme de cette période de stabilisation, le pouvoir effectif de l'Eglise de Lyon se traduit par une assise foncière importante et territorialement très regroupée (une cinquantaine de châtellenies, ainsi que d'importantes possessions à Lyon même), un pouvoir amputé des droits régaliens et une autorité seigneuriale sur Lyon.

Mais cette puissance est quantitativement et qualitativement restreinte par rapport à ce qu'elle était au XIe s. A partir du XIVe s., l'Eglise de Lyon se confrontera à deux pouvoirs rivaux, l'administration royale (sénéchaussée) et l'administration communale.

\section{LE QUARTIER CANONIAL DE SAINT-JEAN}

36 Dans ce contexte, l'enceinte canoniale est le reflet de la puissance réelle de l'Eglise de Lyon. Elle en est à la fois le symbole et le centre vital, puisqu'elle regroupe toutes les institutions spirituelles, juridiques et administratives (résidence du chapitre, sanctuaires, cours de justice, prisons, etc.).

\section{LE CHAPITRE DE L'EGLISE DE LYON}

Constitué depuis l'époque carolingienne (selon un témoignage de l'évêque Leidrade), il est réorganisé au début du XIIIe s. par Renaud de Forez, à l'origine sans séparation des pouvoirs entre le chapitre et l'Archevêque, afin que la souveraineté comtale reste une. Trente-deux chanoines se partagent les revenus du patrimoine foncier, dans le cadre des obéances et des mansions (système qui élimine le risque des ambitions personnelles 
ou familiales sur un territoire précis). A la mort de Renaud de Forez, le chapitre se désolidarise complètement de l'archevêché, lui laissant l'exercice de la justice et l'autorité sur la ville de Lyon et se réservant l'administration des biens extérieurs à la ville, ainsi que la totale propriété du quartier canonial, protégée par des lettres d'immunité du roi de France. Chaque chanoine est théoriquement astreint à partager son temps entre son obéance et sa résidence (selon un régime de vie communautaire souple en logements individuels) à l'intérieur de l'enceinte.

Le chapitre s'est donc constitué comme un monde clos qui s'affirme par la construction d'un rempart. Symbole de cette séparation, de l'isolement du monde ecclésiastique et de son pouvoir, cette muraille est érigée durant l'épiscopat de Guichard de Pontigny (1165-1180): “...Eodem presidente, ambitus murorum claustri ceptus et consommatus est...” (Guigue 1867, p. 121-122). Ce mur d'enceinte, au rôle défensif, enferme tous les bâtiments appartenant au chapitre et à l'archevêché. Cette apparence de château-fort apparaît dans un texte de 1418 : “...de tout temps, le chapitre a eu un cloître en forme de château, auquel était le portail de Porte-Froc construit pour sa défense et celui de la ville..." (Sachet 1914). Cette forteresse est maintenue coûte que coûte du XIIe au XVIe s.; des mentions dans les textes traduisent cet état de fait durant toute la période: limitation du nombre des ouvertures dans la muraille, entretien assidu des murs et des huisseries, enfin fermeture des portes la nuit.

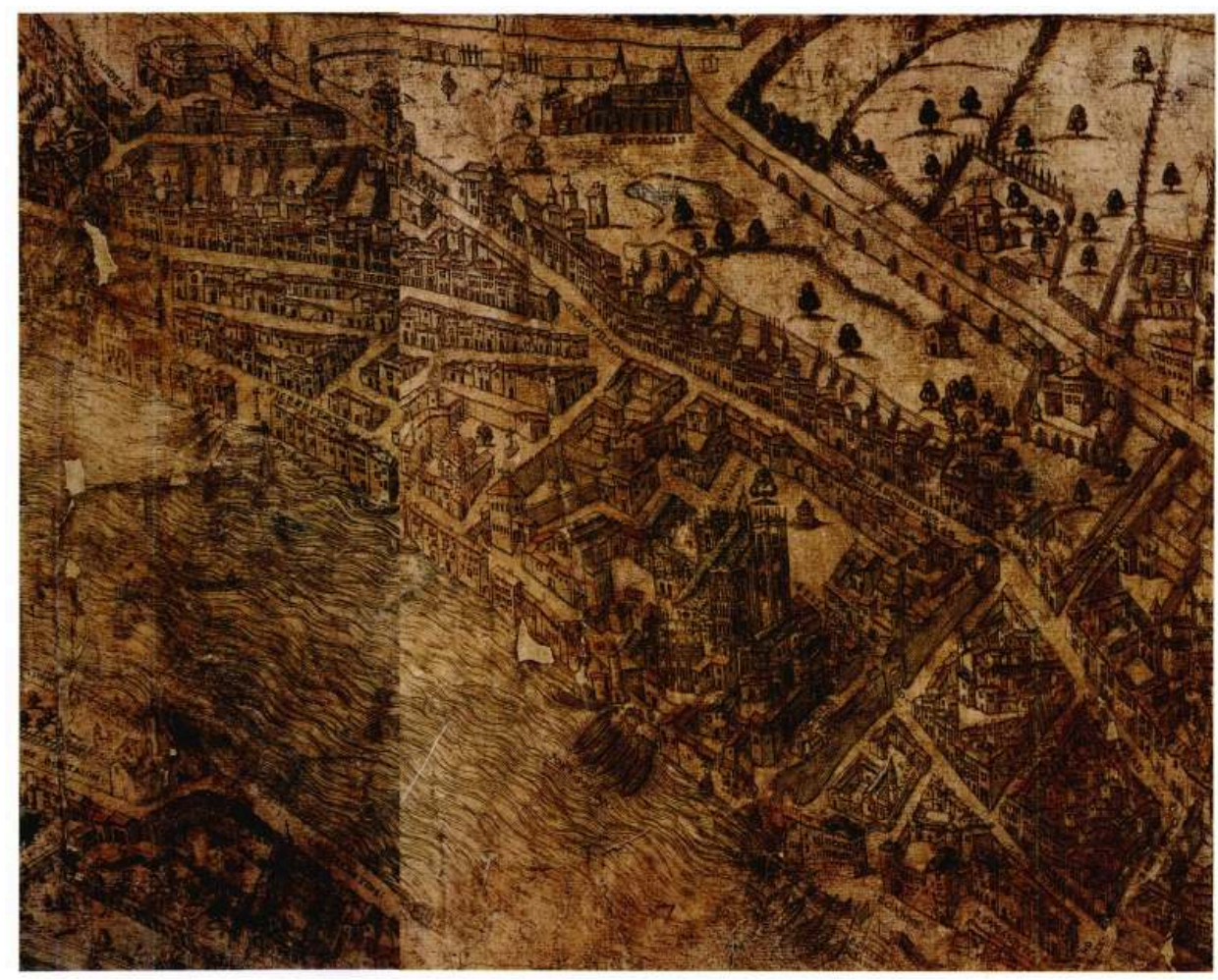

7 - dÉtAIL du PLAN SCÉNOgRAPHIQUE DE LA VILLE DE LYON.

Ce CÉlèbre PLAN EST une RePRÉsentation MINUTIEUSE de LYON AU MILIEU dU XVIE S. IL S'AgIT d'UN PLAN QUI CONCILIE LA gÉométrie et LA PERSPECTIVE ; VUe CAVALIÈRe OU "PORTAIT" de LA VILLE, CE DOCUMENT ICONOgRAPHIQUE, FOURNIT UNE SOMME EXCEPTIONNELLE D'INFORMATIONS. LE COMMANDITAIRE DE L'ÉDITION AINSI QUE LA DATE EXACTE DE SA FABRICATION RESTENT INCONNUS. IL EST CONSERVÉ AUX ARCHIVES MUNICIPALES DE LYON (A.M.L.1.S.165). DANS LE DÉTAIL REPRODUIT CICONTRE, L'ENCEINTE CANONIALE EST BIEN VISIBLE.

39 A partir du XIVe s., l'enceinte prend une valeur de symbole dans la lutte entre la commune des bourgeois et l'Eglise de Lyon, et même dans le rapport de force entre le 
chapitre et l'archevêque. Refusant l'existence de ce nouveau pouvoir politique, les chanoines s'enferment dans leur microcosme.

Ce monde clos se veut uniquement ecclésiastique : des ordonnances répétées au cours des XIVe et XVe s. interdisent le port de l'habit séculier dans le quartier canonial, de même que l'accès des laïcs et à plus forte raison l'installation de marchands (qui en 1550 obtiennent uniquement le droit d'appuyer leurs boutiques au mur extérieur du cloitre).

41 D'un point de vue juridique, depuis les accords avec Philippe le Bel (1307), le quartier canonial est le support territorial des droits du chapitre : sous l'autorité du chamarier, il dispose de sa propre police (assurée par le corps des coponiers) et du droit, entre autres, d'assurer la garde et le guet en cas de danger. Surtout il possède la justice haute, moyenne et basse, dans une juridiction centrée sur le quartier canonial, mais dont les limites en débordent largement (particulièrement au sud : près de $200 \mathrm{~m}$ ).

\section{LE TRACÉ DU MUR D'ENCEINTE DU QUARTIER CANONIAL}

La vision la plus éloquente du tracé de la muraille est donnée par le Plan scénographique de la ville de Lyon, exécuté entre 1558 et 1561 (fig. 7). Comme l'écrit B. Chevallier : “...la vue cavalière... exprime bien le paysage urbain dans toutes ses dimensions, réelles et imaginaires..." (Chevallier 1981).

43 Le tracé de l'enceinte capitulaire, disparue depuis la fin du XIXe s., est encore très repérable dans le parcellaire actuel (fig. 8). Il se présente sous la forme d'un quadrilatère de 185 x 175 m environ accolé à la Saône.

La clôture est représentée encore complète sur le Plan scénographique et mentionnée à maintes reprises dans les textes d'archives. Elle est bornée côté ville par une muraille continue d'imposantes dimensions, crénelée à son sommet. A. Steyert propose un mur de $1,65 \mathrm{~m}$ de largeur sur $6 \mathrm{~m}$ de hauteur (Steyert 1899, p. 361). Un texte de 1628, décrivant la même muraille à l'ouest de la Porte Froc, donne des dimensions sensiblement différentes: “...icelle vieille muraille ayant d'épaisseur dans oeuvre 5 pieds et 4 pouces $(1,80 \mathrm{~m})$ et d'hauteur à l'endroit de ladite maison 24 pieds $(8,16 \mathrm{~m}) . . . "$ (A.D.R. 10.G.595). La muraille proprement dite suivrait le tracé de la rue de la Bombarde (nord), puis de la rue Tramassac (ouest), jusqu'à la rue Carriès, d'où elle traverserait "lîlot Tramassac", rejoignant ensuite l'ancienne rue Mourguet (sud) qui descend jusqu'à la Saône (Sachet 1914) (fig. 9). Elle est apparemment absente le long de la Saône où elle est remplacée par un front de maisons canoniales dans lequel s'enclave le port Saint-Jean. 


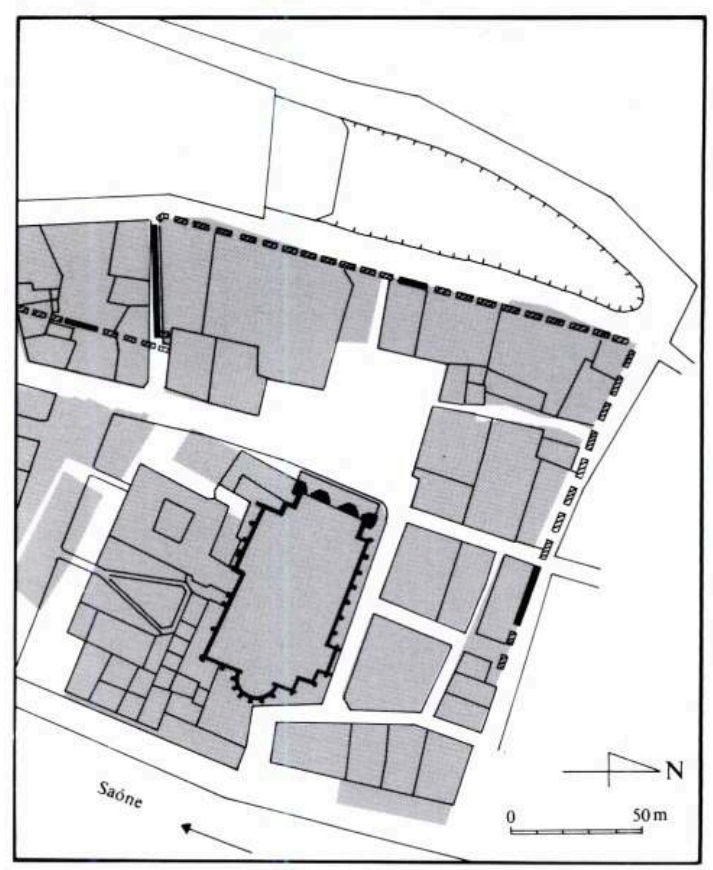

- vestiges du mur d enceinte.

m.mo hypothese

$\square$ cadastre 1830

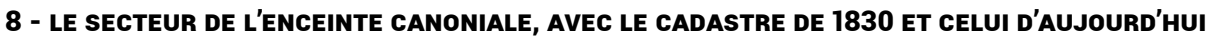

Le rempart est percé de 6 portes assez régulièrement réparties sur son pourtour et dont une seule est fortifiée ; elle est flanquée de deux tours quadrangulaires dissemblables.

En 1562, le mur d'enceinte est partiellement détruit par les Protestants, et, même si le chapitre s'acharne à relever et à conserver l'apparence de cette muraille, de nombreux textes attestent sa dégradation. Les laïcs commencent à s'implanter à l'intérieur du quartier canonial, monde qui leur était jusque-là refusé. Ce mouvement ne cesse de s'amplifier, comme le montrent les nombreux baux de location à des laïcs au XVIIe s., et l'installation d'échoppes dans les rues, puis dans les maisons canoniales au XVIIIe s. De nombreuses portes à usage privé sont percées dans la muraille, surtout après 1650 , alors que les grandes portes ne sont plus réparées depuis 1562. La construction d'un pont sur la Saône en 1634 établit également un contact permanent avec l'extérieur. 


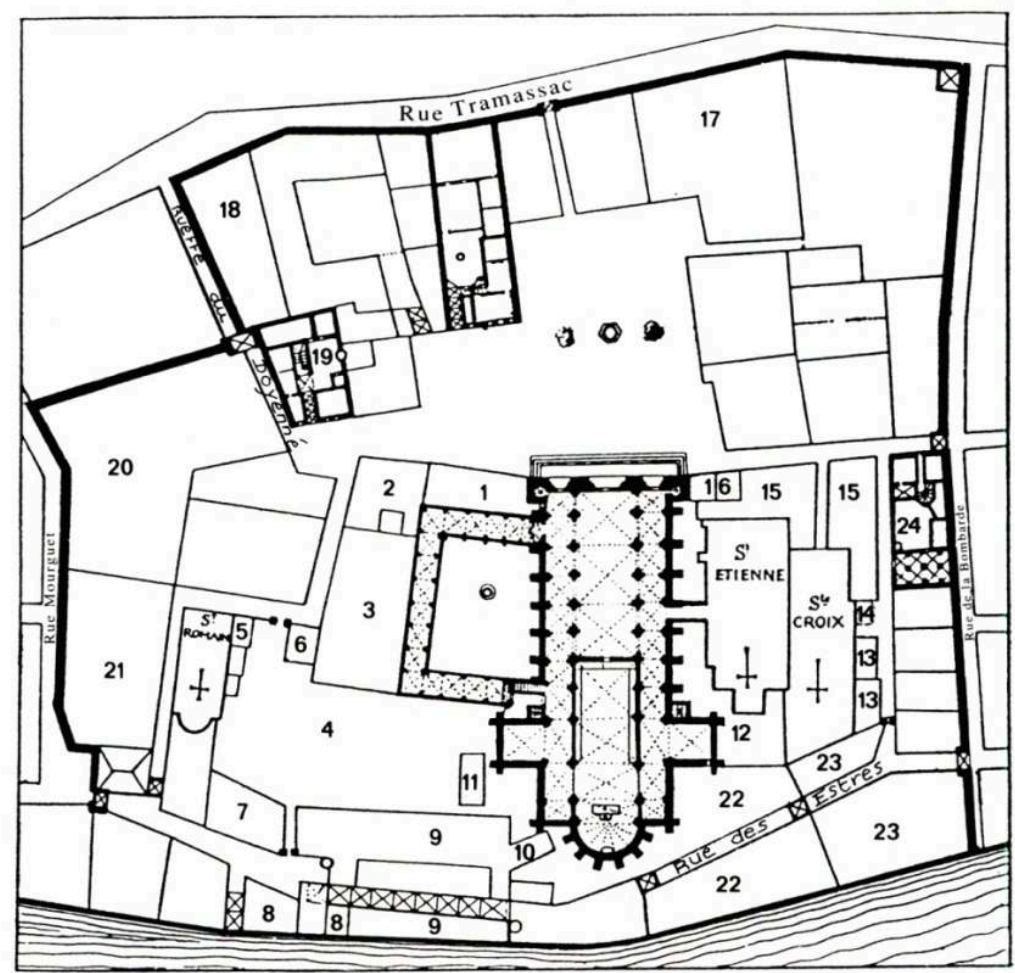

9 - TRACÉ dU MUR d'ENCEINTE dU CHAPITRE de SAINT-JEAN RESTITUÉ PAR A. SACHET (SACHET 1914) 1. MANÉCANTERIE - 2. MAISON DES CLERgEONS - 3. AUDITOIRE DE LA JUSTICE DU COMTÉ - 4. COUR DE L'ARCHEVÉchÉ - 5 ET 6. DÉPENDANCES dE L'ARCHEVÉCHÉ - 7. AUDITOIRE de LA JUSTICE DE L'ARCHEVÉCHÉ - 8. PRISONS de L'ARCHEVÉCHÉ - 9. PALAIS DE L'ARCHEVÉCHÉ - 10. CHAPELLE DE L'ARCHEVÉCHÉ -11. Officialité - 12. PLACE de LA FONDERIE des CLOCHES - 13. PRÉbENDES DE SEMUR - 14. MANILLERIE DE SAINTE-CROIX - 15. CUSTODERIES DE SAINTE-CROIX - 16. LOgES DES OUVRIERS 17. HÔTEL de LA PRÉCENTERIE - 18. HÔTEL d'ALBON - 19. MAISON CANONIALE - 20. HÔTEL DU DOYENNÉ-21. HÔTEL DE L'ARCHIDIACONNÉ - 22. HÔTEL DE LA gRANDE SACRISTIE - 23. HÔTEL DE LA gRANDE CUSTODERIE - 24. hôtel DE LA CHAMARERIE.

Le tracé du mur de l'enceinte que nous proposons pour notre part a été établi d'après l'analyse de plusieurs types de sources, confrontées au tracé donné par le Plan scénographique. Des tronçons du mur ont été découverts lors de fouilles archéologiques (Reynaud 1979) ou repérés en élévation, ainsi le mur nord de la maison du Chamarier (Mounier 1986; Arlaud-Rolland 1990). Les photos anciennes montrent cette muraille conservée en élévation rue Tramassac (fig. 10), et détruite lors de la "catastrophe de Fourvière" en 1930 (Louvet 1981, p. 73).

$\mathrm{Au}$ sud-ouest du quartier canonial, c'est-à-dire à l'intérieur de l'îlot Tramassac, deux tracés ont été proposés : certains historiens englobent les jardins du Doyenné dans le quartier canonial, comme B. Vermorel (A.M.L. 37-II), alors que d'autres, comme A. Sachet, placent ces mêmes jardins à l'extérieur. La première démarche peut s'expliquer par une confusion avec les limites de la juridiction du chapitre, qui ne respectent pas exactement celles du mur de clôture du quartier canonial (A.D.R. $10 \mathrm{G} 615$ et 614 ). A cet endroit précisément, les jardins du Doyenné font partie de la juridiction du chapitre, ce qui ne signifie pas forcément qu'ils sont à l'intérieur de l'enceinte canoniale. La fouille a confirmé l'existence d'un angle de l'enceinte. Ses deux cotés ; nord/sud et est/ouest ont en effet été repérés, l'un dans l'îlot Tramassac, l'autre dans l'axe de la rue Carriès. 


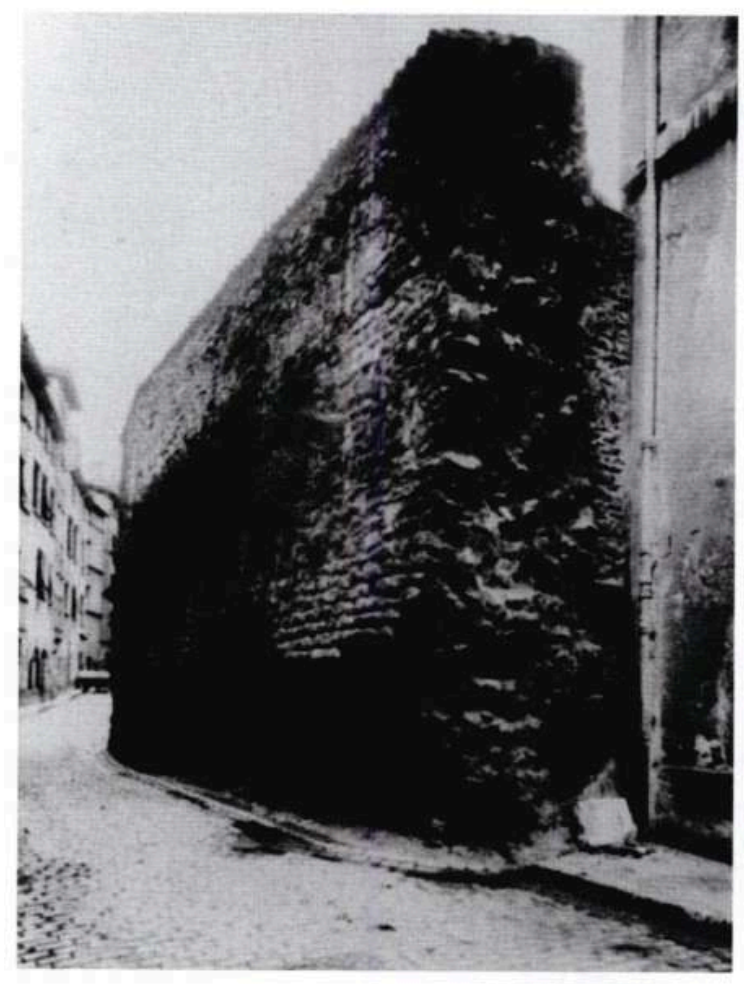

10 - Le mur de L'enceinte RUe tramassac (Cliché y. LOUVET) (LOUVET et PerRAUdin 1981)

\section{L'HABITAT MÉDIÉVAL}

L'opportunité de l'opération archéologique réalisée dans la partie occidentale du quartier Saint-Jean a permis de percevoir ce que F. Verhaeghe (Verhaeghe 1991) appelle "la troisième composante essentielle du monde urbain médiéval...., l'espace civil" et de comprendre le rôle du quartier canonial comme moteur de la dynamique urbaine à l'extérieur de l'enceinte, au moment des luttes politiques entre l'archevêque et le comte de Forez.

50 Les informations recueillies ont pu être croisées avec celles rassemblées en d'autres points de la rive droite de la Saône ${ }^{4}$ et permettent de confirmer des hypothèses quant à la mise en place du parcellaire, le lotissement du quartier, la maison (matériaux, techniques de construction, organisation de l'espace domestique, implantation des bâtiments dans la parcelle, bâtiments annexes) ainsi que sur les composantes de l'espace civil public : la rue, l'approvisionnement en eau etc.

L'existence de la muraille introduit une rupture dans l'évolution du bâti du quartier. A l'extérieur, se met en place un mode de gestion de l'espace où se développe l'habitat civil. Il s'est d'abord développé à l'intérieur de vastes tènements appuyés sur la rue dont l'origine du découpage est encore mal définie. Le morcellement de ces tènements sous la contrainte des partages et des ventes aboutit à la mise en place d'un parcellaire en longues bandes étroites, dit "en touches de piano". A l'intérieur, des maisons particulières sont construites dans le périmètre proche de la cathédrale sur de grandes parcelles, comme l'hôtel du Doyenné qui ne respectent pas les orientations du Haut Moyen Age. 

l'alignement des façades et des percées à travers la ville, d'autres parce qu'elles ne correspondent pas aux critères d'urbanisme ni aux besoins de cette époque. A la place des demeures ecclésiastiques, des maisons de rapport sont construites ou aménagées avec boutique sur rue au rez-de-chaussée. avec la création de la gare Saint-Paul et de ses accès, la construction de deux lignes de funiculaires depuis Saint-Jean, l'aménagement des quais de Saône et la construction des ponts. Malgré cela, ce quartier est en déclin durant le XIXe s. et une grande partie du XXe s. jusqu'à la publication de la loi Malraux sur les secteurs sauvegardés. Celle-ci marque un tournant en matière de protection du patrimoine et se traduit par un intérêt croissant pour ce quartier ancien qui conduira bientôt à sa restauration. l'intérieur de celle-ci, un escalier en vis permet l'accès à la maison sur rue comme à la maison sur cour par un système de galeries reliant les deux à chaque étage. Dans les textes, les mentions de "maison haute et basse" ou "haute, moyenne et basse", indiquent deux à trois niveaux d'élévation. selon les endroits, pour lequel il est actuellement difficile de proposer une explication plausible. Il est cependant possible de formuler trois hypothèses: soit il s'agit d'un remblaiement à mettre en relation avec la construction du mur du cloître (mais, dans ce cas-là, comment expliquer qu'aucune pente ne soit perceptible à la fouille, ni aucun talus contre ledit mur?), soit il s'agit d'un amoncellement dû à une occupation intense et aux transformations du bâti qui y sont liées, soit il faut le mettre en relation avec des phénomènes hydrologiques liés au "petit âge glaciaire" des XIVe et XVe s.

a été vérifié dans d'autres villes, comme à Tours sur des maisons des XIIe et XIIIe s. : “...zone d'occupation constante depuis l'Antiquité, le sol s'est exhaussé et les rez-dechaussée se sont enterrés et sont devenus des premiers étages de caves..." (Catalogue d'exposition Tours 1980, p. 26). Des éléments comme un seuil dans un mur de façade arasé (au niveau du sol des caves actuelles) et l'accumulation des niveaux d'occupation sont les témoins de cette surélévation du niveau de sol à Tramassac. Les caves ont par la suite été voûtées, sans doute pour supporter un surcroît de charge dû aux constructions d'étages supplémentaires. Pour rétablir une hauteur suffisante (une voûte a environ 0,80 à $1 \mathrm{~m}$ d'épaisseur), le sol de la cave est creusé ; ceci explique d'une part le hiatus qui existe dans la chronologie des sols, et d'autre part, l'absence, jusquelà énigmatique, de fondations pour les maisons qui ont été étudiées dans ce quartier.

\section{LE DÉMANTĖLEMENT DU QUARTIER CANONIAL} maison par maison. Son emplacement devient l'objet d'une urbanisation intensive afin de l'intégrer au reste de la ville: la percée de la rue du Doyenné, puis de l'avenue A. Max, l'élargissement de la rue de la Brèche et de la rue Carriès en sont quelques exemples.

Nombre de maisons canoniales sont démolies et reconstruites; certaines à cause de 


\section{NOTES}

2. Nous n'utiliserons pas le terme habituel "cloître", trop ambigu pour désigner l'ensemble des bâtiments ecclésiastiques, mais "enceinte canoniale" ou quartier canonial.

3. Les fouilles de la place des Célestins (Arlaud 1992), située sur la Presqu'île à Lyon, en face du quartier Saint-Jean, ont permis de mettre au jour des niveaux contemporains et des structures identiques à celles décrites dans le présent volume. L'étude détaillée de ce site permettra d'établir des comparaisons et peut-être de faire évoluer la problématique propre à cette époque.

4. Thèse en cours. L'habitat civil à Lyon, histoire et archéologie (rive droite de la Saône et Presqu'île). C. Arlaud, sous la direction de J.-M. Pesez. 


\section{Deuxième partie. Étapes de l'urbanisation}

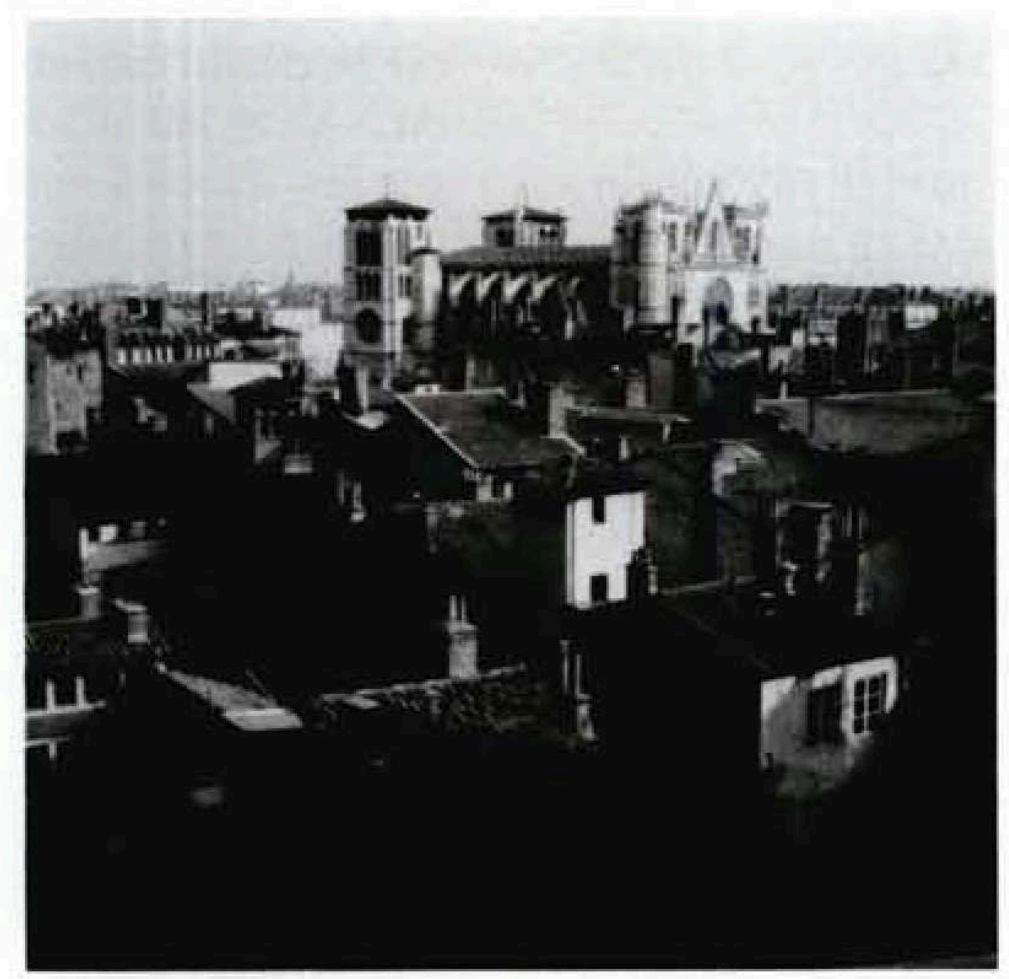

1 Les observations archéologiques de l'îlot Tramassac sont une fenêtre ouverte sur le passé du cœur de la ville médiévale de Lyon. C'est une fenêtre bien étroite au premier abord : quelques milliers de mètres carrés seulement, sur l'étendue desquels le sous-sol a été souvent remanié, au gré des reconstructions. Néanmoins, comme on a essayé de le montrer dans la première partie, ces observations apportent des connaissances nouvelles non négligeables sur l'évolution du quartier Saint-Jean. Elles le doivent à l'application de méthodes désormais classiques en matière d'archéologie urbaine: enregistrement minutieux et rigoureux des données livrées par la fouille, pluridisciplinarité des approches et confrontation systématique avec les sources 
écrites. L'inventaire raisonné des traces archéologiques observées, consigné dans les pages qui suivent, est le résultat de cette démarche. Il constitue le corpus des preuves de la démonstration au sens ou l'entendent les historiens. Parmi celles-ci, on a privilégié la description des données de terrain relatives à la stratigraphie et à l'organisation spatiale des vestiges. C'est l'objet de cette deuxième partie. Leur interprétation se nourrit aussi du résultat d'un certain nombre d'études spécifiques, d'une part des analyses de mobilier qui seront détaillées dans la troisième partie, d'autre part de données d'archives relatives à la topographie ancienne du quartier.

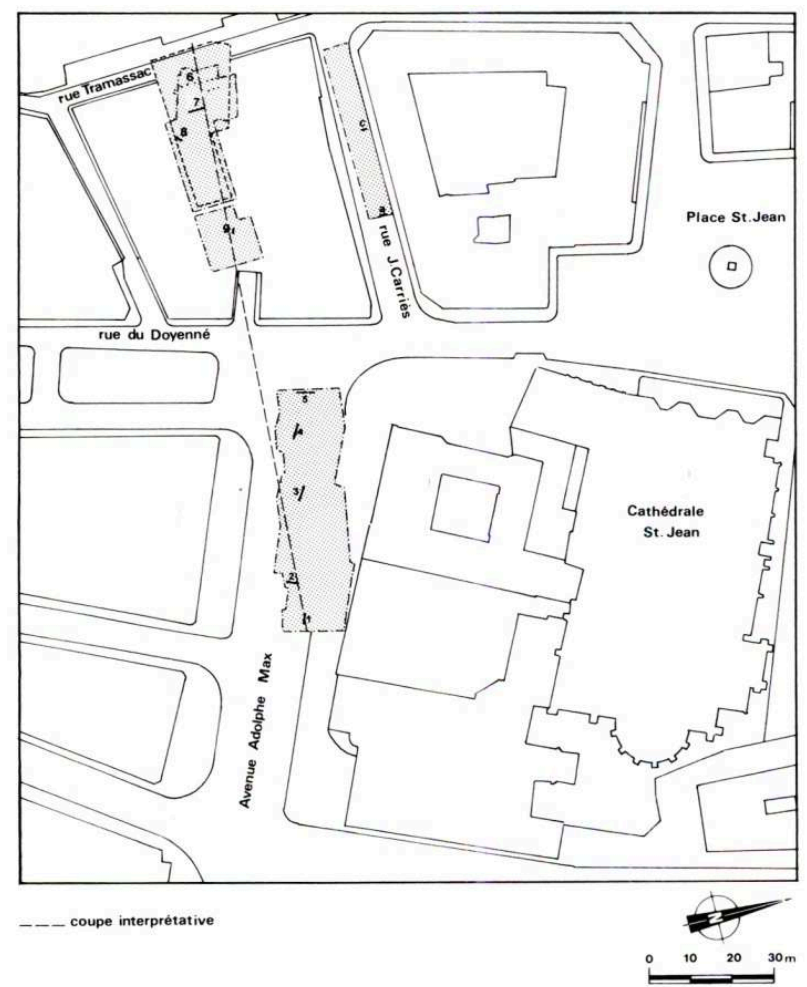

11 - Localisations des sites fouillés et des observations géomorphologiques

\section{TOPOGRAPHIE ET PREMIERS AMÉNAGEMENTS}

\section{LE SITE AU DÉBUT DE L'ANTIQUITÉ}

2 L'interprétation des données du sous-sol provenant d'une vingtaine de sondages carottés réalisés dans la phase préparatoire à la construction du métro a permis de dégager les grandes lignes de la stratigraphie fluviatile (fig. 11).

Entre le pied du coteau de Fourvière et la Saône actuelle, les alluvions fluviatiles reposent à une altitude voisine de 150-155 m N.G.F. sur un "conglomérat de base" dont un niveau d'arène granitique situé au contact du socle du Massif central. Le matériel alluvial de base est un remplissage sablo-graveleux "de type Saône", de couleur rose à rouge, également qualifié par les sondeurs de "sable grossier" (fig. 12). Le sommet de cette formation se tient aux environs de 156-157 m entre la rue Tramassac et le quai de la Saône. Cette formation a été retrouvée sous la Presqu'île à une altitude équivalente.

4 La différenciation topographique se fait dans les formations sédimentaires sus-jacentes. A l'ouest, un chenal d'axe N N O.- S S E. est nettement dessiné entre la me Tramassac et 
la partie ouest de l'avenue $\mathrm{A}$. Max : le talweg était à une cote légèrement inférieure à $156 \mathrm{~m}$ (155,70 $\mathrm{m}$ au sondage 472$)$. Ce bras semble avoir été large d'une centaine de mètres. Les alluvions qui le remplissent sont épaisses de 6 à $8 \mathrm{~m}$ et sont décrites comme des sables, des "graves sableuses de couleur rouge", parfois noirâtres et à passées limoneuses, surmontées d'un épais remblai urbain (environ $6 \mathrm{~m}$ ).

Entre ce bras de Saône et le cours actuel de la rivière, le sable de Saône précédemment décrit est surmonté par un niveau de galets rhodaniens à matrice sableuse mixte (Saône et Rhône), culminant aux environs de 162,50 à 163,50 m N.G.F. A l'est d'une ligne passant par la bibliothèque et la rue Monseigneur la Varenne, cette couche s'abaisse vers la Saône actuelle (162-159 m) en dessinant une berge ; mais cette topographie peut être relativement récente si l'on admet que l'érosion latérale de la Saône post-antique a pu retailler la rive. Cette couche caillouto-sableuse forme donc un banc entre le paléochenal situé à l'ouest, au pied de la colline de Fourvière, et la rive droite de la Saône actuelle.

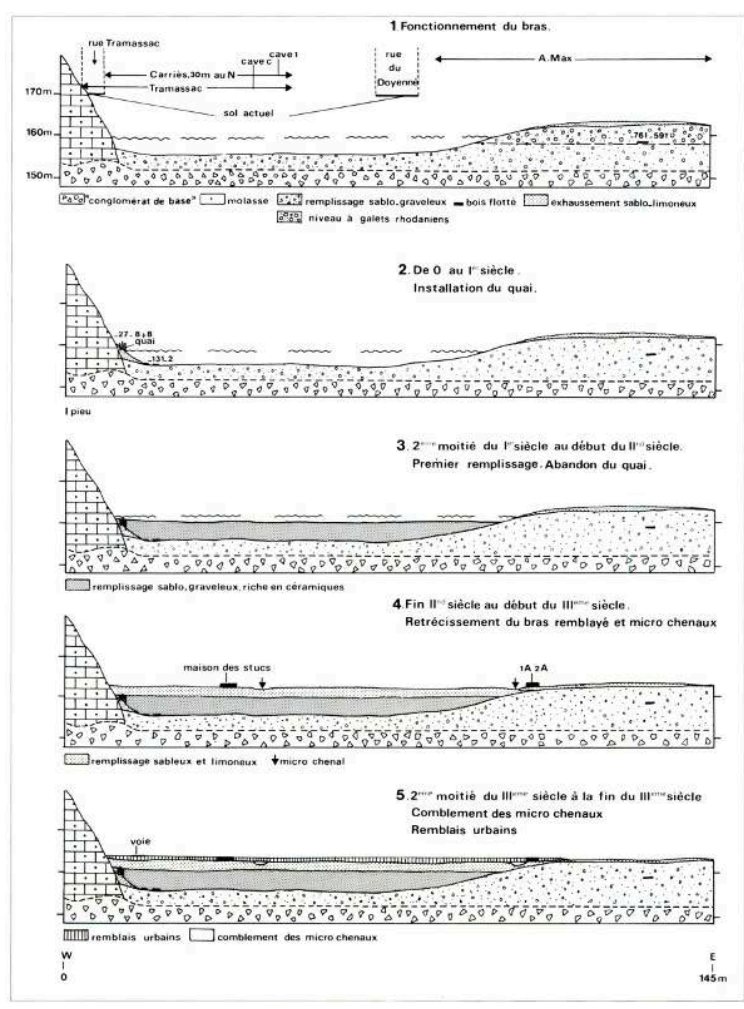

12 - Coupes interprétatives, étapes de remblaiement du bras de la Saône

La structure des dépôts suggère une évolution relativement complexe à l'Holocène (voir fig. 6). Les alluvions sableuses de base ne sont pas encore datées : leur position sous les alluvions caillouteuses du Rhône suggère que la Saône a construit un vaste épandage confluant avec le Rhône sur la rive gauche du fleuve actuel. L'incision du lit fluvial que cette disposition démontre fait pencher pour une mise en place au milieu de l'Holocène (Bravard et alii 1989) mais cette hypothèse reste à démontrer.

7 Le niveau de galets rhodaniens qui forme le cœur du quartier Saint-Jean (entre 150-155 et 162,5-163,5 m) a probablement été mis en place à l'époque protohistorique. En effet, ce dépôt a pu être daté grâce à la découverte d'un tronc d'arbre à la cote $158,50 \mathrm{~m}$ sur le chantier de l'avenue A. Max (datation dendrochronologique de 761-591 av. J.-C.; abattage pas antérieur à 561 av. J.-C. $)^{5}$ Le matériel caillouteux est soit contemporain du 
tronc subfossile, soit postérieur si l'on admet que le tronc a été déposé par les eaux, puis repris ultérieurement par les crues avant d'être fossilisé. Quelle que soit la solution retenue, ce niveau se serait donc mis en place entre le premier Age du Fer et le 1er s. av. J.-C. L'hypothèse d'une construction alluviale remontant au VIe s. av. J.-C. est renforcée par le fait que d'autres découvertes de troncs subfossiles, à proximité de ce site, ont fourni des fourchettes chronologiques similaires (Becker et alii 1989).

8 La signification morphodynamique de cette construction alluviale est particulièrement importante pour la connaissance du site de Lyon. Elle montre que la dynamique du Rhône se métamorphose au premier Age du Fer avec une phase de très fort transport caillouteux et d'exhaussement généralisé. Il est fort possible que ce niveau caillouteux ait raviné les sables roses sous-jacents dont la cote sommitale actuelle ne reflèterait pas le niveau originel. Cette masse grossière, épaisse de 6-7 $\mathrm{m}$ au moins, envahit le site de la Presqu'île actuelle et celui de Saint-Jean.

9 La Saône, dont la charge était moins grossière et le régime plus régulier, a été plaquée contre l'escarpement de Fourvière, ce dont témoigne le chenal repéré par les sondages et déjà signalé par A. Audin. Les dimensions de ce chenal laissent penser qu'il écoula l'essentiel, voire la totalité, des eaux de la Saône. La question se pose en effet de savoir si la Saône a toujours existé à son emplacement actuel. Les constatations précédentes plaident en faveur de l'existence d'un chenal unique situé au pied de Fourvière, auquel cas le banc caillouteux de Saint-Jean aurait été le prolongement occidental de la Presqu'île actuelle. Les fouilles dont il est ici rendu compte ne permettent pourtant pas d'exclure la possibilité qu'un bras de la Saône ait séparé le site de Saint-Jean et la Presqu'île durant la protohistoire. L'apport essentiel concerne les modalités de la fermeture progressive du chenal situé au pied de la colline de Fourvière dans l'Antiquité ; les coupes observées et l'analyse granulométrique des sédiments récoltés ont permis de préciser l'évolution du banc de Saint-Jean et les différentes séquences sédimentaires de remblaiement du bras à l'époque antique dans les limites qu'impose une fouille de sauvetage programmé.

10 Les observations réalisées sur les trois chantiers, avenue A. Max, rue Tramassac et Carriès, ont permis de décrire la mise en place du remblaiement de ce bras de Saône ; cinq étapes ont pu être distinguées (fig. 12).

11 Au Ier s. av. J.-C., (fig. $\left.12 \mathbf{n}^{\circ} \mathbf{1}\right)$ le bras de la Saône situé entre le coteau de Fourvière et Saint-Jean avait une largeur d'une centaine de mètres et un fond aux environs de 156 $\mathrm{m}$. Des bois flottés récoltés au fond du chenal vers la cote $156 \mathrm{~m}$ N.G.F. ont fourni des dates d'abattage échelonnées entre 131 et 2 av. J.-C. par dendrochronologie (Arc 7/R109 séquence 9010 TRA). Ces découvertes signifient que le bras a conservé sa profondeur jusqu'aux environs de notre ère, ou que des remaniements contemporains de cette époque ont recreusé le chenal partiellement remblayé en y abandonnant ces bois.

12 A l'est, à l'emplacement de Saint-Jean, se tenait un banc caillouteux culminant vers 162,5-163,5 m sur le site de l'avenue A. Max.

13 Les coupes relevées à l'ouest du chenal de la Saône (fig. 13) ont permis l'observation d'un épandage limono-sableux déposé sur le banc de galets. La base du dépôt se tient aux environs de la cote 162,90 m N.G.F. et possède une épaisseur comprise entre 50 et $90 \mathrm{~cm}$. Ces coupes, les plus éloignées du bras, présentent une stratification régulière, mais non parallèle, avec localement une structure "en vagues" traduisant des 
phénomènes d'érosion par les courants de débordement. Les lits inférieurs sont épais de 5 à $20 \mathrm{~cm}$ et sont plus sableux que la couche limono-sableuse qui coiffe l'ensemble.
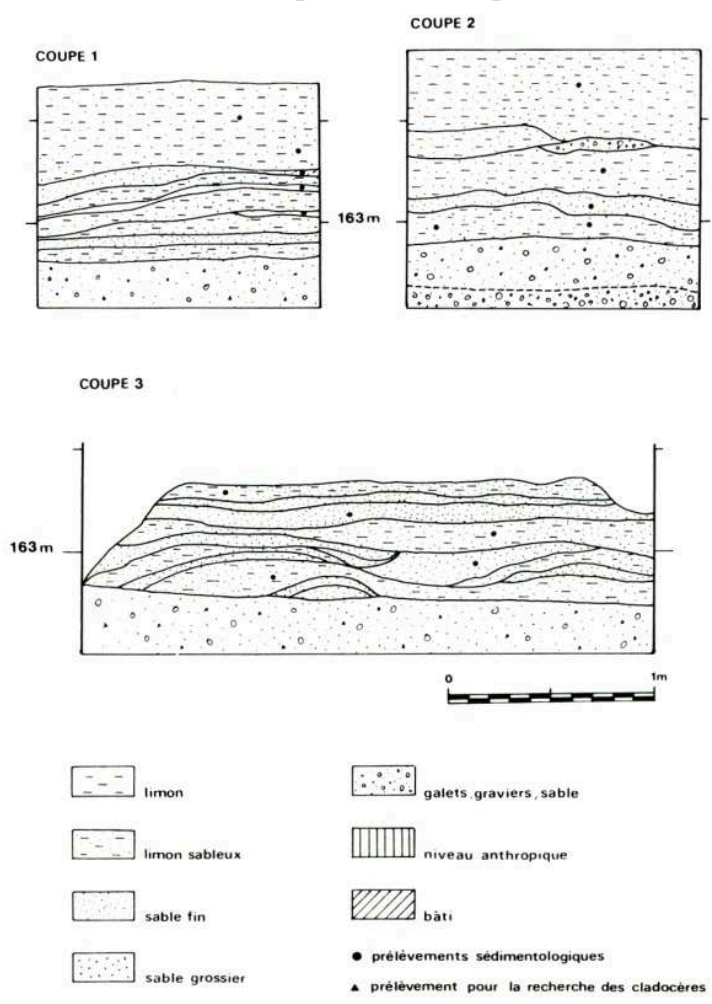

13 - Coupes réalisées sur le chantier avenue A. Max

14 La coupe 3, proche du bras, présente deux structures superposées. A la base, une séquence épaisse de $30 \mathrm{~cm}$ à structure en vagues non parallèles avec surface de réactivation ; au sommet, une séquence de lits parallèles et quasi-horizontaux.

Pour ce revêtement, la technique, dite "de l'image $\mathrm{CM}^{\mathrm{N}}$ ", confirme l'hypothèse selon laquelle ces sédiments sont des dépôts de crue (fig. 14). Des dépôts de sable caractéristiques de bancs ou de berges proches du cours d'eau alternent avec des dépôts plus fins (courants lents) caractéristiques de sommets de bancs ou d'une plaine d'inondation.

16 La pétrographie et la morphoscopie de ces sédiments fins de recouvrement permettent de trancher en faveur d'une influence prépondérante de la Saône.

Le seul dépôt qui puisse avoir été mis en place par une crue du Rhône est une lentille sableuse rencontrée dans la partie est du chantier, à une quinzaine de centimètres au-dessus des galets (elle est caractérisée par une majorité de quartz translucides ou laiteux, par la rareté des grains oxydés, par des minéraux noirs abondants, par l'abondance des grains non usés à cassure fraîche, et enfin par la rareté des grains émoussés luisants).

L'essentiel du revêtement a donc été mis en place par la Saône, mais il a fallu, pour émettre cette hypothèse, dépasser le stade d'un examen visuel des lits qui sont tantôt rosés, tantôt gris ; ces alternances pourraient en effet laisser supposer une influence alternée de la Saône et du Rhône. En réalité, les lits de couleur rose à jaunâtre sont formés de dépôts issus de suspensions graduées; leur couleur est due à la rareté du mica noir (évacué par lévigation), à la présence de quartz oxydé rose ou concrétionné et blanchâtre. Au contraire les lits sombres sont constitués de sédiments issus de suspensions uniformes ; les grains sont en général polyminéraux (destruction des arènes granitiques), ou constitués de mica noir, de quartz clair et 
peu usés.

Cette distinction illustre un principe connu qui est le triage des sédiments par les courants (enrichissement relatif des suspensions uniformes en mica noir, en grains polyminéraux) et la moindre usure des suspensions uniformes par rapport aux suspensions graduées. Ces remarques ont une importance méthodologique dans la mesure où les fouilles archéologiques peuvent mettre au jour des lits sédimentaires isolés et difficiles à interpréter en l'absence de structure: le recours à la granulométrie, à la pétrographie et à la morphoscopie peut éventuellement faciliter les interprétations.

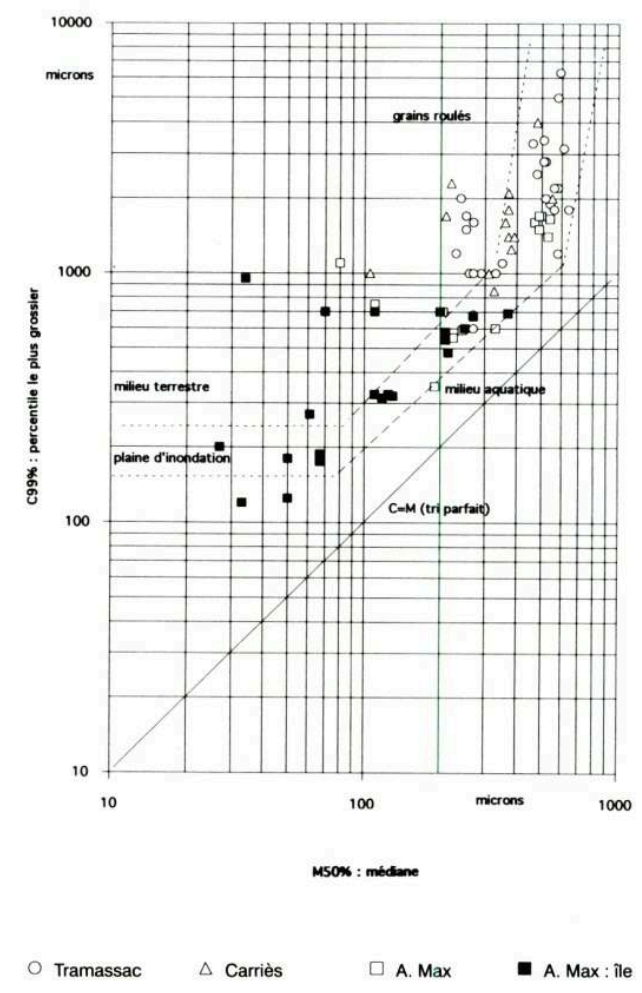

14 - Image granulométrique CM des sédiments fins de Saint-Jean

La méthode de l'image CM permet de mettre en relation les variations de texture d'un sédiment, obtenue par l'analyse granulométrique, avec le processus de transport et de dépôt. Deux valeurs sont extraites de la courbe granulométrique :

- M, la médiane est la valeur du diamètre pour 50 \% de l'échantillon ; elle définit la structure d'ensemble du dépôt.

- C, mesure la compétence maximale du courant ayant transporté les particules ; il correspond à la valeur du diamètre pour 99 \% de l'échantillon. Ainsi, chaque échantillon est représenté par un point. La position de ce dernier sur l'image formée d'un nuage de points, détermine un mode de transport (plus ou moins turbulent) auquel est associé un mode de dépôt. A chaque type de dépôt, correspond une unité de la plaine alluviale : lône, plaine d'inondation, berge, chenal, etc...Cette méthode d'interprétation de la granulométrie des sédiments permet donc de reconstituer les unités morphologiques des milieux alluviaux fossilisés.

\section{UN APPONTEMENT DE LA FIN DU PREMIER SIĖCLE AV. J.-C. (PÉRIODE 1)}

17 L'aménagement des berges du bras de la Saône correspond à la période 1.

18 Des vestiges d'un aménagement des berges du bras de la Saône ont été découverts dans la partie ouest de l'îlot Tramassac, juste au pied de la colline de Fourvière et à $10 \mathrm{~m}$ sous la surface du sol actuel, soit à 160 m N.G.F. ; il s'agit d'une structure en chêne composée de pieux de section circulaire, d'environ $0,25 \mathrm{~m}$ de diamètre supportant un platelage de 
troncs équarris de section rectangulaire dont les dimensions sont de $0,25 \mathrm{~m} \times 0,35 \mathrm{~m}$ en moyenne. L'analyse dendrochronologique a daté un premier groupe d'échantillons provenant des pieux et du platelage de 27 av. J.-C.; un deuxième groupe, de même provenance, est daté de 8 av. J.-C. et un échantillon isolé de 18 ap. J.-C. (Arc. 87/R.109D). La possibilité de réemplois ne permet pas de trancher entre une construction d'un seul jet en 18 ap. J.-C., ou une construction précoce ( 27 av. J.-C.) suivie d'agrandissements et de consolidations. Cet appontement, dont les pieux portent des traces d'érosion dues au courant, peut être un quai aménagé ou une voie de circulation sur berge (fig. 15); elle atteste de toute manière un aménagement de la rive droite de la Saône pour un usage régulier.

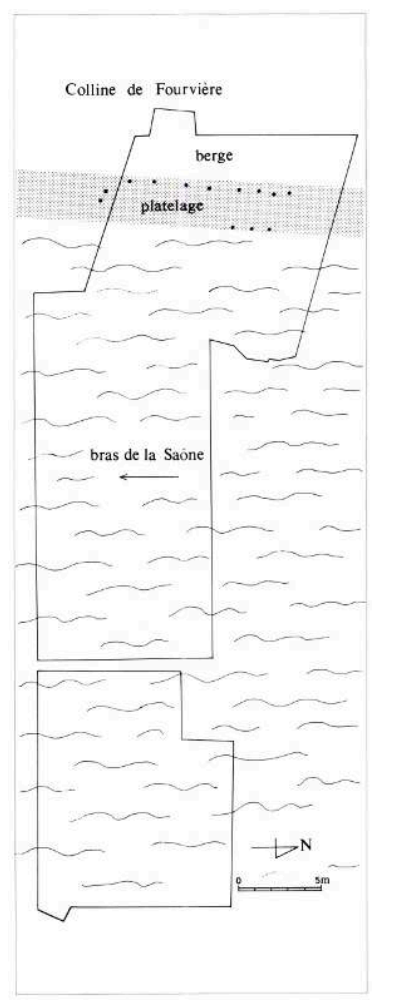

15 - Plan des vestiges de la période 1

La confrontation des analyses du matériel céramique, à fin de datation, avec les résultats de l'étude dendrochronologique n'a pas pu être réalisée car le mobilier céramique, prélevé dans les niveaux sablonneux de l'aménagement des berges, constitue un échantillon trop mince pour permettre d'envisager une datation.

La position des pieux permet de proposer l'existence d'un appontement dominant un plan d'eau dont le niveau moyen pourrait être proche de $159 \mathrm{~m}$ et sujet à d'amples fluctuations lors des crues. Le matériel fluviatile sableux dans lequel sont enfoncés les pieux pourrait témoigner d'un commencement du remblaiement du chenal sur sa marge ouest au début du Ier s. av. J.-C. (fig. 12 n²).

Pour conclure sur cette période, il faut donc imaginer le paysage de la façon suivante: sur le sommet de la colline de Fourvière s'étendait la ville haute avec ses quartiers d'habitation, ses bâtiments administratifs, religieux et culturels; l'habitat s'est également installé sur les pentes sud et ouest. Au pied de la colline, la Saône, large d'une centaine de mètres et profonde de $3 \mathrm{~m}$, était longée par un appontement d'une longueur inconnue. Sur la rive gauche, les crues débordantes déposaient des lits de 
sable qui exhaussaient progressivement le vieux banc caillouteux de mise en place rhodanienne.

\section{DU ler AU IIle SIÈCLE}

\section{LE COMBLEMENT DU BRAS DE LA SAÔNE (PÉRIODE 2)}

Le comblement du bras occidental de la Saône commença dès la fin du 1er s. ap. J.-C. et se poursuivit jusqu'à sa disparition totale à la fin du IIIe s. tandis que se mettait en place un bras oriental, à l'emplacement actuel du fleuve. Ce remplissage a laissé trois séquences sédimentaires.

\section{LES PREMIERS REMPLISSAGES}

La première séquence, observée dans l'îlot Tramassac lors des interventions de sauvetage ponctuelles, a été repérée entre 156 et $161 \mathrm{~m}$ (fig. $12 \mathbf{n}^{\circ}$ 3). Elle est constituée, à la base, d'une importante couche très hétérogène qui correspond au dépôt de la charge de fond. La texture de cette couche prouve qu'à ce moment-là, le courant de la rivière était important; en effet, ce niveau est constitué principalement de sédiments grossiers d'origine rhodanienne (galets du Rhône) sans doute remaniés par la Saône (la matrice des galets est formée de sable de Saône) (fig. 16 a). Ensuite, se déposèrent une succession de couches à granulométrie décroissante vers $161 \mathrm{~m}$ : sables grossiers, limons (fig. 16 b). Cette gradation vers le haut est le résultat d'une évolution du fonctionnement hydraulique qui se caractérise par des courants de plus en plus faibles. Des couches anthropisées enrichissent l'évolution naturelle du remplissage.

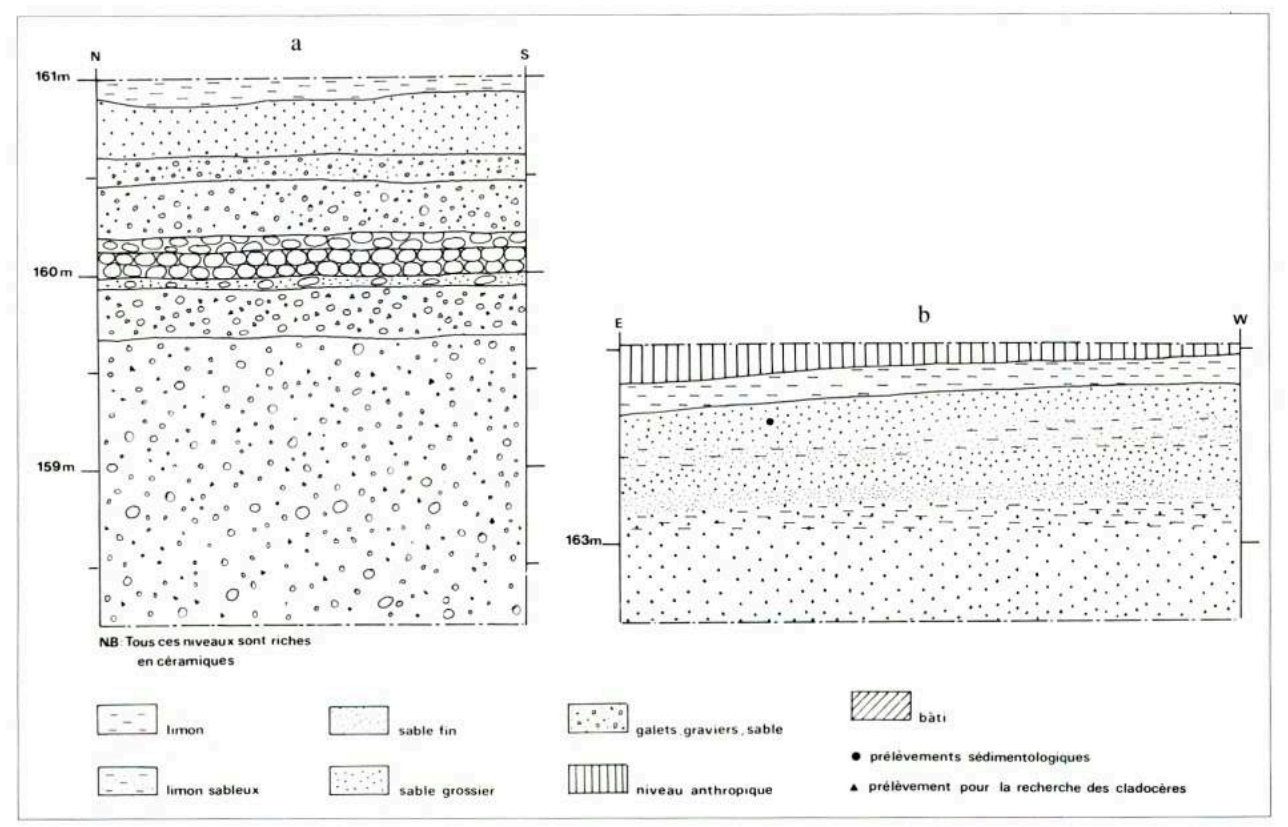

16 - Relevés schématiques des premiers remplissages du bras de la Saône, îlot Tramassac

La première phase active de ce remblaiement, constitué principalement de cailloutis, graviers et galets de diamètre moyen $8-10 \mathrm{~cm}$ (maximum :20 $\mathrm{cm}$ ) sur plus de deux mètres mérite une explication.

Il aurait été intéressant de faire une pétrographie des galets, mais les conditions de travail en sauvetage n'ont pas permis cette étude. Une hypothèse s'impose tout de 
même, pour expliquer cette masse caillouteuse.

Il semble que la Saône remaniait le matériel rhodanien du lit majeur, et que la part provenant du bassin de la Saône était faible. Plusieurs indices autorisent une telle interprétation:

- à l'aval de la Croix-Rousse, la pente du lit majeur, imposée par le Rhône, avoisine $1 \%$;

- jusqu'au 1er s. ap. J.-C., le contrôle des berges par l'homme devait être limité, si bien qu'une recharge latérale par érosion de berge était possible au détriment des dépôts caillouteux présents sur les rives du cours d'eau.

Dans tous ces niveaux, le mobilier céramique était important, mais les conditions de travail n'ont permis qu'un ramassage en vrac: le matériel n'étant pas stratifié par rapport aux séquences sédimentaires, la datation proposée après l'étude de la céramique - deuxième moitié du Ie s. au début du IIe s. ap. J.-C -, englobe les deux phases de remblaiement (active et calme). Il est à noter que les céramiques observées dans ces phases de remblaiement ne présentent pas de bords "lavés", c'est-à-dire usés par l'eau et les sédiments. Elles ont donc été piégées sans être "entraînées" par des flux hydriques. Il est probable que leur abondance exceptionnelle traduit déjà à cette époque la volonté de l'homme d'assainir cette zone en accélérant le processus naturel de remblaiement.

Ce dépôt, qui entraîna un exhaussement du fond du lit, occasionna sans doute l'abandon du quai de la période 1 : les sédiments atteignent la cote $161 \mathrm{~m}$ N.G.F. environ et fossilisent l'appontement. Celui-ci ne remplissait plus sa fonction, qui n'aurait ainsi duré qu'un siècle (du début de notre ère au début du IIe s.).

\section{LE RÉTRÉCISSEMENT DU BRAS PAR REMBLAIEMENT}

La deuxième séquence sédimentaire (fig. $12 \mathbf{n}^{\circ}$ 4) est composée d'une succession de niveaux sableux et limoneux qui traduisent un nouvel affaiblissement du courant. Le dernier niveau minéral est composé de sable grossier, ce qui constitue la manifestation d'un retour relativement violent du courant. L'ensemble est mis en place entre la deuxième moitié du IIe s. et la première moitié du IIIe $s$.

Les observations effectuées dans l'îlot Tramassac (Tramassac VI) ainsi que l'étude du site de l'avenue A. Max et de la rue Carriès ont permis de préciser les phases sédimentaires entre 162 et 163-163,5 m N.G.F. Celles-ci correspondent à la fin du remplissage alluvial du bras de Saône et une évolution de l'île Saint-Jean.

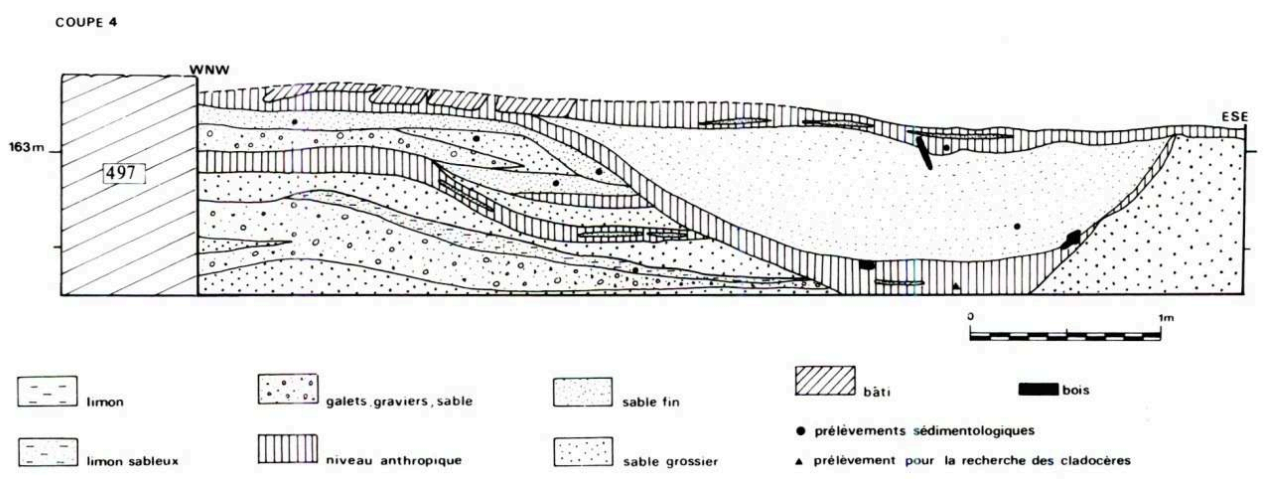

17 - Coupe réalisée sur le chantier avenue A. Max

L'îlot Tramassac, cette séquence sédimentaire, qui se tient entre 162,80 et 163,50 m. est constituée d'une phase à lits sableux, dans lesquels le matériel céramique est 
toujours présent; ces lits sont scellés par une phase fortement chargée en matière organique. L'alternance de plusieurs lits de sable grossier et de sable fin traduit une succession de phases de crue et de décrue; cependant, la diminution de l'énergie fluviale se traduit par raffinement de la séquence vers le haut, par la plus grande régularité du litage subhorizontal à léger pendage vers l'est et, enfin, par la sédimentation de matière organique compacte sur une dizaine de centimètres d'épaisseur; ceci démontre l'arrêt progressif des apports minéraux allogènes. L'absence de sédiments fins, tels les limons, entre les niveaux sableux et la couche organique, laisse penser à un arrêt relativement brutal du passage des crues. Ce phénomène pourrait être lié à une action humaine en amont, le barrage de ce bras d'eau permettant par exemple de stabiliser définitivement cet espace afin de l'occuper.

Au-dessus de la couche organique, un remblai argilo-sableux fut apporté volontairement afin d'assainir l'espace et de le rendre propice à une installation de longue durée.

Rue Carriès, la stratigraphie visible dans le secteur 1 révèle un pendage S.E.-N.O. sur l'emplacement de la berge d'un chenal résiduel comblé ultérieurement.

Avenue A. Max, la coupe 4 (fig. 17), la plus significative, est transversale au bras. Elle met en évidence un chenal résiduel près d'un mur (US 497) qui pourrait être interprété comme une digue. Ce chenal recoupe une série de couches à structure entrecroisée. Cette structure dénote l'alternance de phases à courants érosifs et de phases plus calmes avec accumulation (type fin de crue). Ces phases de remaniement et dépôt sont entrecoupées de périodes de répit hydrologique permettant la sédimentation de matière organique; l'intercalation de minces lentilles sableuses dans ces derniers lits signale des phases mineures de reprise d'activité hydrodynamique.

La granulométrie des dépôts est d'une grande complexité, car une partie du remplissage semble avoir été réalisée par l'homme. La fouille archéologique de l'avenue A. Max a montré que le bâtiment le plus ancien du site a été construit sur une butte ; la couche sur laquelle il reposait a livré un lot de céramiques datables du troisième quart du IIe s. attestant la fréquentation de cet espace et probablement l'apport de remblais (Villedieu 1990). Il est possible que ces remblais expliquent la présence de couches caillouto-sableuses mêlées à des fragments de céramiques souvent roulés; ces lits à inclusions grossières traduisent de toute façon l'intervention d'un courant violent, capable de remanier les matériaux les plus lourds et de leur conférer une structure de dépôt "naturel". Des apports successifs ont donc pu contribuer à remblayer le bras à partir du pied de l'escarpement de Fourvière ; le bâtiment le plus ancien aurait été construit dans la deuxième moitié $\mathrm{du}$ IIe s. sur les bords d'un chenal résiduel; cependant, il n'est pas exclu que les courants de crue aient été capables d'éroder les matériaux grossiers de l'île, par effet de sapement latéral.

Les observations sédimentologiques démontrent ainsi que, jusqu'à la fin du fonctionnement de ce chenal, le courant a gardé une vitesse suffisante pour remanier les alluvions (fig. 18), y compris des lits de galets: ce chenal a donc constitué un obstacle tardif à l'installation du bâti. Elles démontrent également que la séquence de remblaiement du bras s'est achevée entre le milieu du IIe s. et la première moitié du IIIe s. ap. J.-C. Le sommet des alluvions culmine à 163,50 m, sensiblement au même niveau que le banc Saint-Jean recouvert par des dépôts sablo-limoneux d'exhaussement. La couche organique observée dans l'îlot Tramassac souligne une dépression bien marquée, dans l'axe central du bras. A la fin du IIe s. ap. J.-C., le bras s'était rétréci à une cinquantaine de mètres de largeur. La circulation des courants de crue dans des chenaux résiduels ravinait le remblaiement en place sur une profondeur d'un mètre ; deux chenaux ont été observés, le long de l'île Saint-Jean et dans la coupe a de la rue 
Carriès (voir fig. 11). Il semble que la stratigraphie observée à l'est de la (coupe 9) rue Tramassac, mette en évidence le même micro-chenal que celui repéré rue Carriès.

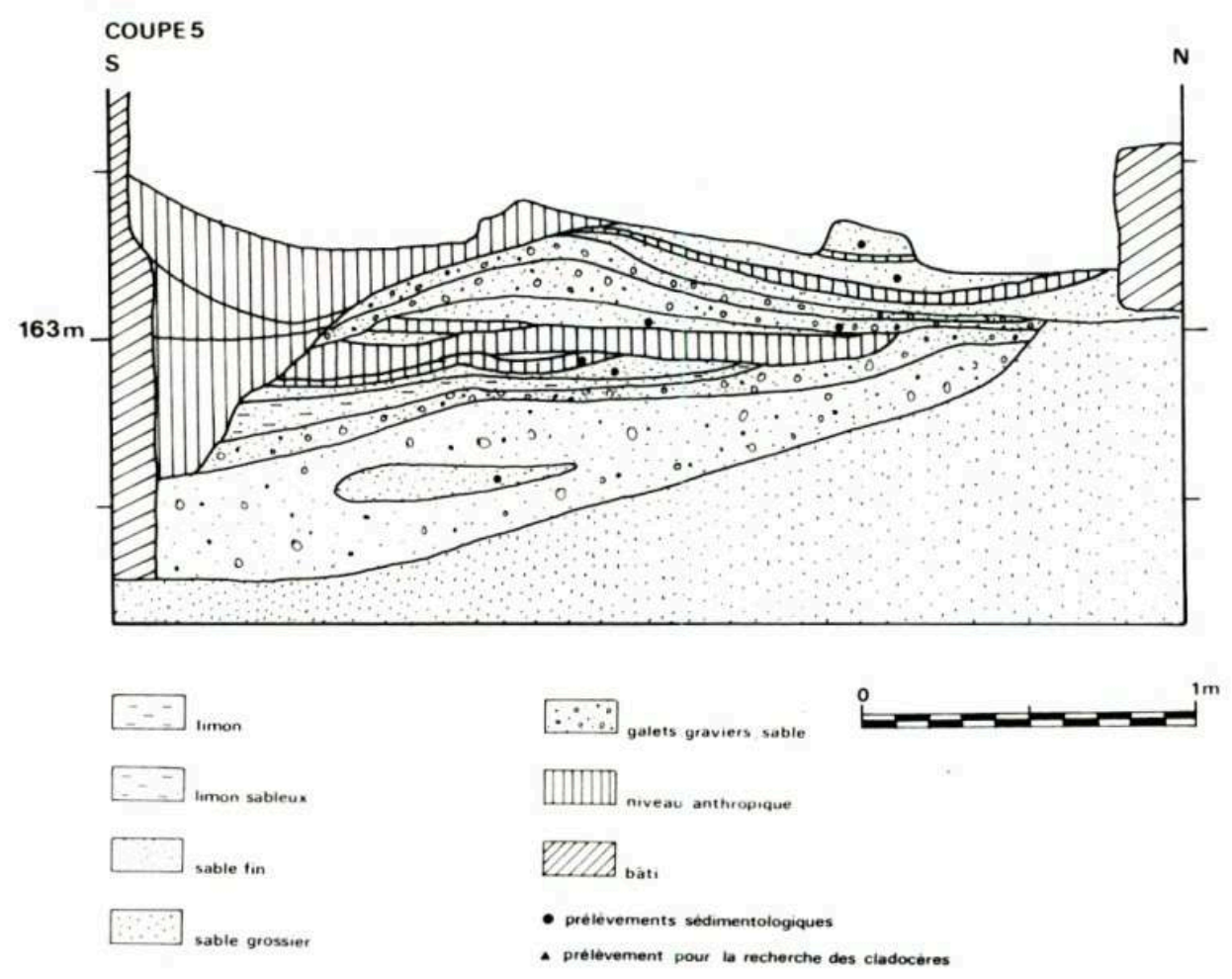

18 - Coupe réalisée sur le chantier avenue A. Max

Ainsi le bras situé entre Lourvière et l'île Saint-Jean a conservé tardivement un paysage de flaques résiduelles à l'étiage de la Saône : cette "lône" servait de dépotoir et pouvait être colonisée par quelques plantes aquatiques. Lors des crues et des hautes eaux, le bras doublait la Saône (cours principal) et assurait l'évacuation des eaux : un courant rapide nettoyait les fonds et reprenait les matériaux caillouteux et sableux. A la décrue les matériaux se redéposaient en provoquant un remblaiement progressif des chenaux résiduels.

Sur les marges de cette dépression entaillée par les chenaux, les hommes commençaient à s'installer. D'une part, avenue A. Max, des bassins se déversaient dans le chenal résiduel qui fonctionnait encore, d'autre part, un bâtiment, repéré rue Carriès, est daté de la première moitié du IIIe s. ap. J.-C. La séquence sédimentaire repérée sur la coupe $C$ (fig. 19) est en effet recouverte d'un remblai daté du milieu du IIe s., et enfin une maison repose sur des remblais de la fin du IIe s. au début du IIIe s. Ainsi, pendant cette phase où des chenaux résiduels de crue fonctionnaient épisodiquement, l'homme commençait à coloniser les marges de l'ancienne Saône à partir des deux rives relativement stabilisées. 


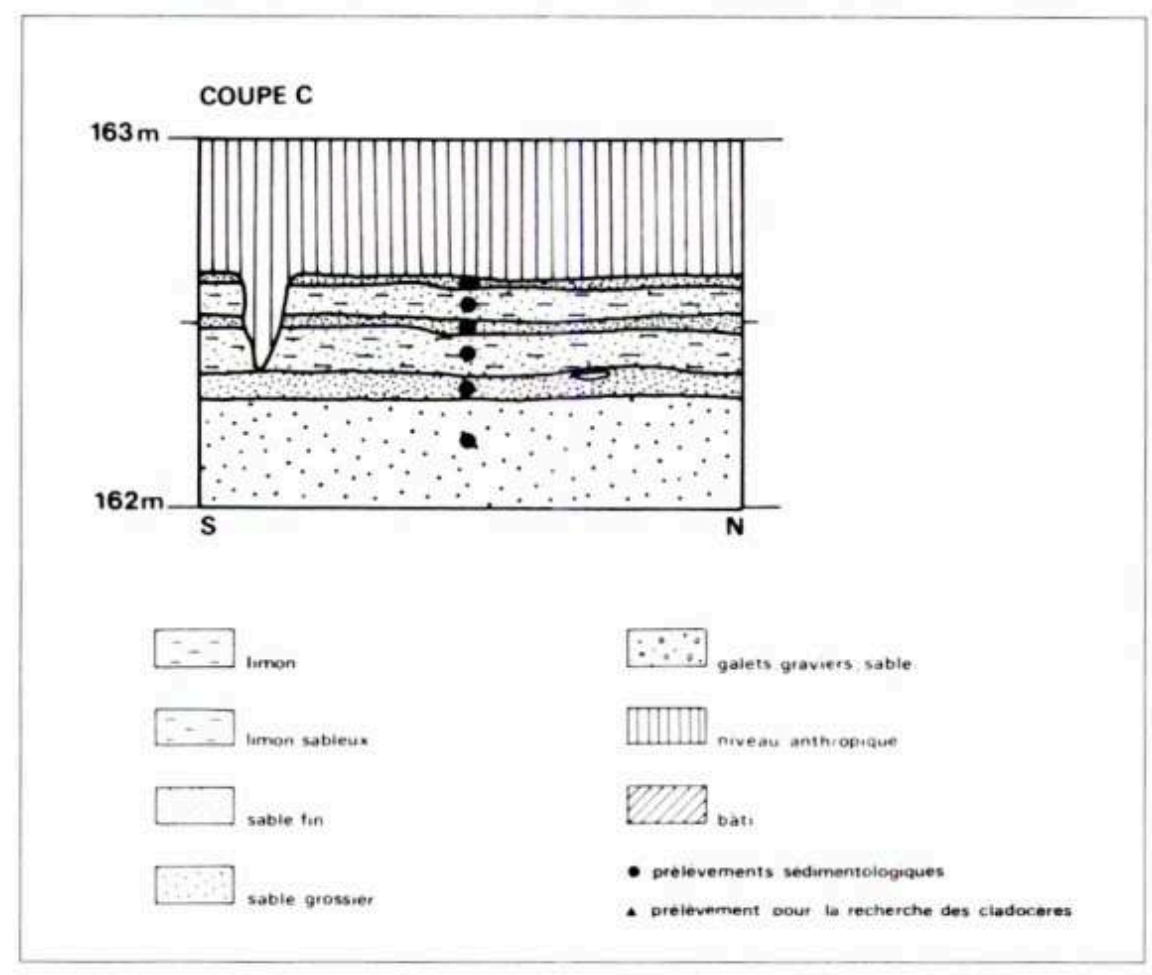

19 - Coupe réalisée sur le chantier rue J. Carriès

\section{LE COMBLEMENT DES CHENAUX RÉSIDUELS}

Des observations réalisées dans l'avenue A. Max, l'îlot Tramassac et la rue Carriès, permettent de rattacher des phases sédimentaires à la phase finale d'assèchement des chenaux résiduels (fig. $12 \mathbf{n}^{\circ}$ 5). En effet, l'étude du matériel céramique trouvé dans le remblaiement alluvial des chenaux montre que ceux-ci ont fonctionné jusqu'au début du IIIe s.

\section{Avenue A. Max}

31 La coupe 4 permet d'observer le comblement du chenal-résiduel repéré sur ce site (fig. 17). Ce micro-chenal est rempli de sable grossier disposé en lits parallèles et incurvés ; son fond est constitué par une couche sablo-limoneuse sombre, très organique, riche en matériel archéologique de rebut. Le comblement progressif de ce chenal est résumé sur une coupe (fig. 20). La stratigraphie des alluvions permet de reconstituer le banc de galets $(162,80 \mathrm{~m})$ plongeant vers l'ouest sous les sables du chenal. L'exhaussement sablo-limoneux puis limoneux atteint une épaisseur de 1,20 m; certains indices laissent penser que la séquence est à peu près intacte et qu'il n'y a pas eu d'importants décapages de surface. Le remplissage sableux du chenal est constitué de grains roulés sur le fond et atteint la cote 163,60 m N.G.F. ; son sommet est donc à une altitude voisine de celle du sommet de l'exhaussement limoneux. La juxtaposition à une altitude comparable de dépôts issus de processus de roulement et de dépôts plus anciens, issus de suspension, suggère qu'un aménagement a pu séparer les deux domaines, à savoir le bras encore actif et l'île, qui ne recevait plus de matériaux lors des crues. 
De fait, la coupe (fig. 20) montre entre autres, la présence d'un mur d'une largeur supérieure à 1 mètre à la base, construit en blocs de micaschiste liés par un mortier blanc-verdâtre désagrégé; il s'avéra qu'il était ancré sur le banc de galets, après dégagement des sables de recouvrement. La face des blocs, tournés vers le chenal résiduel et dégagés du mortier originel, était enduite d'une pellicule d'argile noire d'origine apparemment aquatique. Cette argile a fait l'objet d'une étude complémentaire sur la recherche de restes de cladocères, qui sont des micro-crustacés considérés comme de bons descripteurs des conditions écologiques.

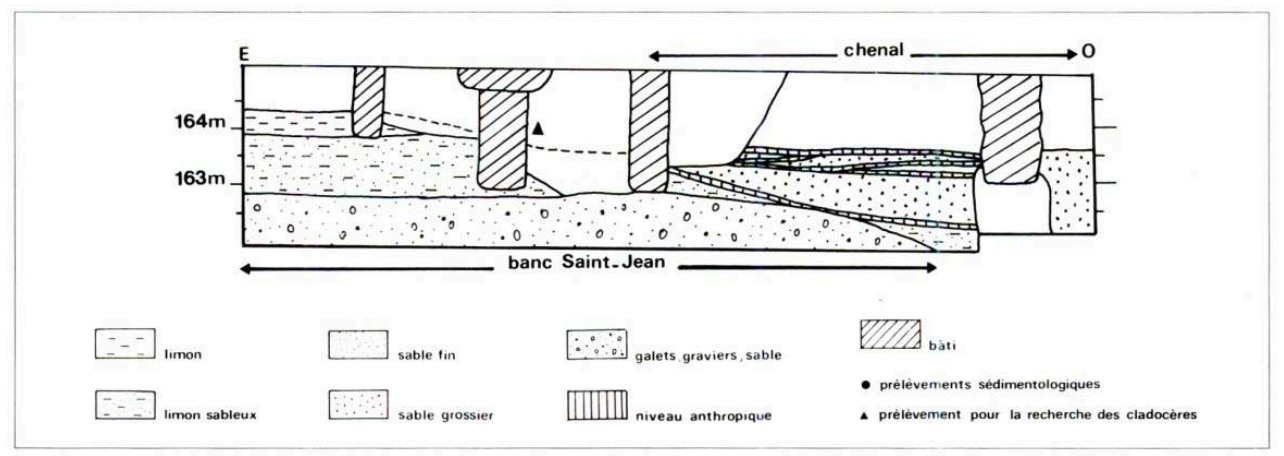

20 - Coupe interprétative réalisée sur le chantier avenue A. Max

Cette argile a livré une capsule céphalique de Biapertura affinis (chydoridae) parfaitement conservée. L'abondance est très faible pour la quantité de sédiment analysée $\left(2 \times 0,05 \mathrm{~cm}^{3}\right)$, mais peut cependant laisser supposer un léger courant, le caractère temporaire des eaux permettant l'installation et le développement de cette espèce.

Par ailleurs, cette analyse a été effectuée sur deux échantillons de sédiments prélevés dans le chenal résiduel :

- une argile à débris d'ossements et céramiques, de type dépotoir de fond de lône (éch. 1) ;

- une argile limoneuse prélevée dans la couche archéologique 2262 sous le bâtiment le plus ancien (éch. 2).

Le premier échantillon a démontré que le biotope était aquatique une partie de l'année, si ce n'est toute l'année, et que les eaux étaient temporairement stagnantes. Le deuxième échantillon n'a pas livré de cladocères. Cette absence est probablement due à l'existence d'un courant trop vif.

36 La technique utilisée est particulièrement adaptée à l'étude des bras morts et des lônes à faible courant ; on pouvait donc espérer obtenir des informations sur l'écologie du bras. En fait, l'analyse des sédiments a démontré que ce chenal résiduel a toujours servi de passage à des courants de crue rapides, même si l'écoulement avait un caractère temporaire.

\section{Îlot Tramassac}

La coupe 9 dégagée (Tramassac III) entre 162 et 163 m N.G.F., montre une stratification entrecroisée formée de lits de sables plus ou moins grossiers, voire limoneux. Des niveaux très anthropisés s'intercalent entre les niveaux sableux; ces derniers renferment eux-mêmes des tessons de céramique et des fragments de brique. Ces couches ont pu être ainsi datées de la seconde moitié du IIIe s. ap. J.-C. Sur le diagramme CM (voir fig. 14), la position des échantillons prélevés dans ces niveaux 
démontre que les sédiments ont été roulés et que d'autres plus fins ont été transportés en suspension graduée. Le courant est donc encore assez vif durant cette phase.

La datation de ces niveaux nous conduit à les rattacher à la phase de comblement des micro-chenaux, même si la topographie ne nous permet pas de dire que nous sommes précisément dans l'un des chenaux résiduels.

\section{Rue Carriès}

La coupe a (voir fig. 11) a révélé une stratigraphie au pendage N.-S., dans le sens de l'écoulement, mais aussi un léger pendage S.E.-N.O. qui pourrait correspondre au bord d'un chenal résiduel. Ce chenal est comblé par une série de niveaux anthropiques et de couches sableuses entre 162 et 163,20 m. Comme sur le site de Tramassac, l'analyse des échantillons confirme le passage d'un courant vif. Les sédiments étaient transportés essentiellement par roulement. Le remplissage de ce chenal résiduel est daté de la deuxième moitié du IIIe $s$. grâce au mobilier céramique des niveaux les plus profonds du sondage. L'espace occupé jusqu'alors par le bras de Saône devint propice à une installation humaine après l'apport d'un remblai d'assainissement.

\section{La maison aux stucs}

Avant même son comblement final, un bâtiment fut construit sur la berge ouest de ce micro-chenal. En est conservé un mur, orienté sud-est/nord-ouest, construit en briques crues et recouvert d'enduits peints décorés; les fragments de ces enduits ont été trouvés au pied du mur, face décorée contre terre. Le registre décoratif des peintures des parois et la hauteur déduite d'après ces mêmes décors et d'après les stucs de la corniche indiquent qu'il s'agit d'une pièce d'un bâtiment public ou d'une salle d'apparat d'une grande demeure privée (fig. 21). La fourchette chronologique donnée par l'étude du mobilier de l'ensemble des couches (remblai de préparation, sol et démolition) permet de dater la construction de ce bâtiment de la première moitié du IIIe s. et son abandon de la fin du IIIe s. Sa destruction semble correspondre à des modifications importantes dans ce secteur.

41 Ces observations nous montrent que les chenaux résiduels ont fonctionné jusqu'à la première moitié du IIIe s., date à laquelle le remblaiement progressa pour conduire à un assèchement total. L'homme accéléra le processus de comblement de ce bras de la Saône par des apports de remblais. Cette stabilisation du terrain permit de l'investir à partir des berges en direction du talweg. La Saône avait alors adopté définitivement son tracé actuel. 


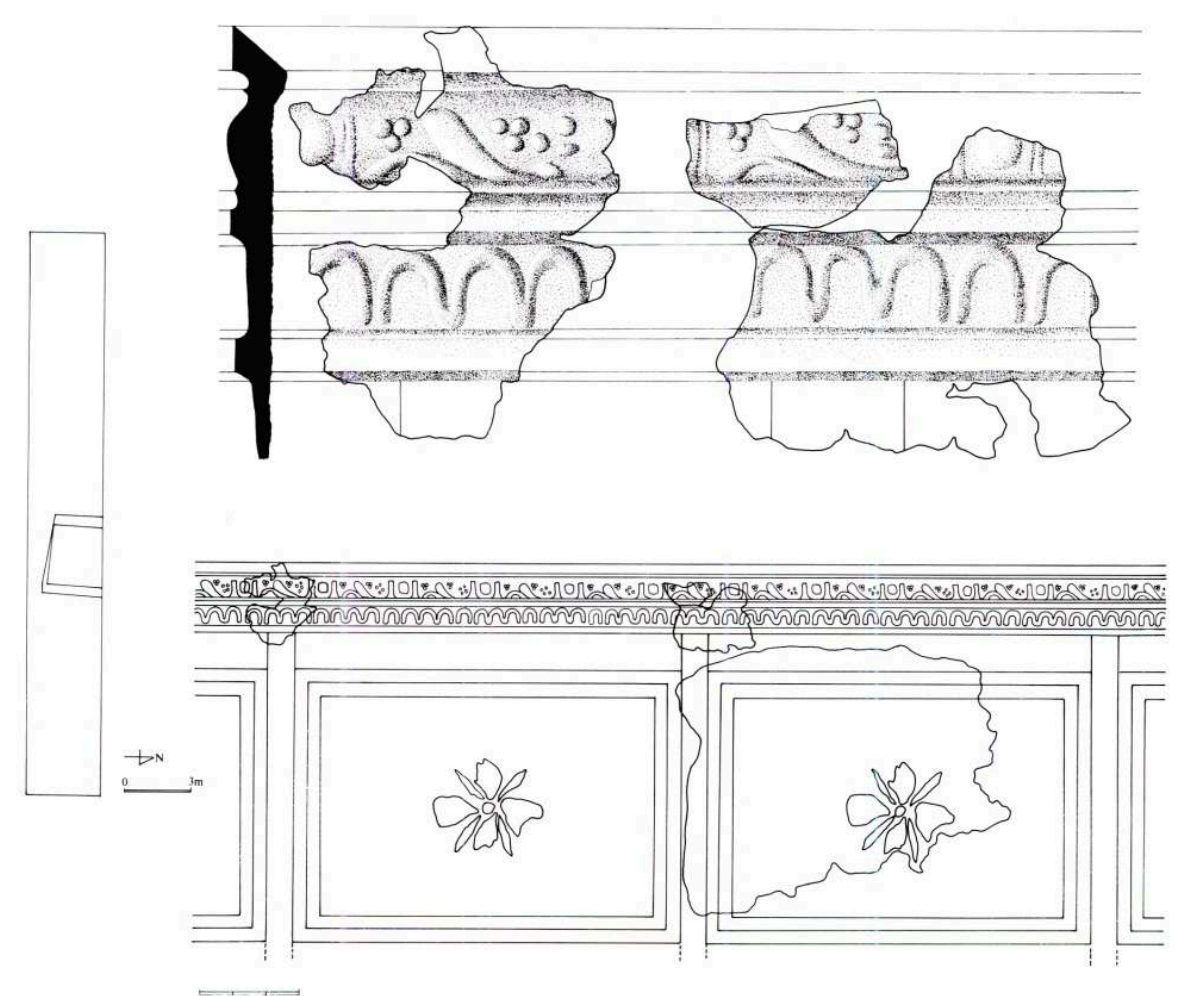

21 - Relevé et restitution des enduits peints décorés de la "maison aux stucs". Situation de la "maison aux stucs" dans le chantier rue J. Carriès

\section{UNE ORGANISATION DE L'ESPACE EN RELATION AVEC LE COMPLEXE EPISCOPAL (PÉRIODE 3)}

L'occupation extensive du site débute au IIIe s., date à laquelle le bras de Saône est définitivement comblé. Elle fait donc suite à une occupation plus sporadique, repérée rue Carriès et sur l'île Saint-Jean (Villedieu 1983).

Pendant deux siècles (IVe - Ve s.), le sud-ouest du groupe épiscopal est habité sans interruption. Cette occupation s'accompagne de nombreux remaniements (trois phases de construction), mais il s'agit chaque fois de structures de facture soignée (constructions en pierres liées au mortier, sol de mortier de tuileau...) qui constituent pour nous autant de preuves de l'intégration de cet espace au groupe épiscopal.

Dans la partie ouest du site, de la terre est apportée de manière à établir une terrasse relativement horizontale qui restera assez meuble. Des dalles de granite de gros module sont installées à son sommet, formant un niveau de voie (US 278) (fig. 22, 23). Au nord, les dalles sont toujours en place, mais masquées par un mur postérieur (US 892-895 période 14). 


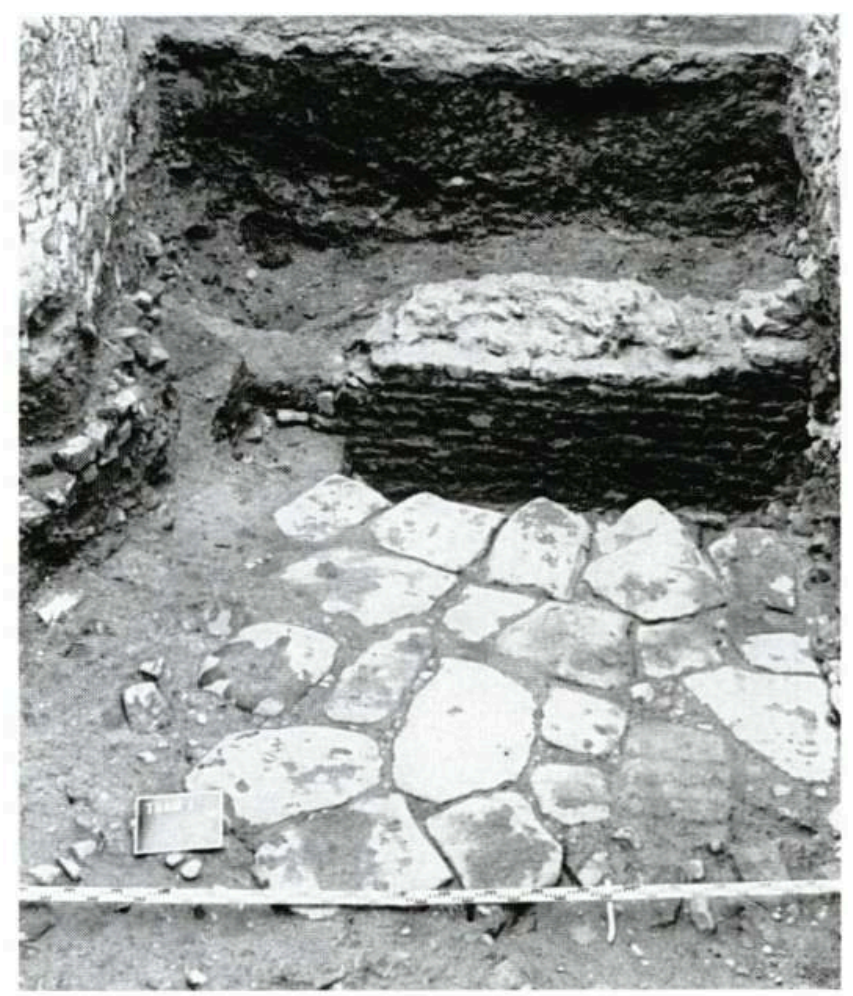

22 - Voie coupée par le mur 209 et sa tranchée de fondation circulation ait été repéré à l'ouest de la zone de fouille de la rue Carriès, permettent d'attribuer une orientation nord/sud à l'axe de cette voie. Par contre, l'installation postérieure d'un mur empêche de connaître son extension à l'est, et donc sa largeur. Le matériel extrait des remblais d'aménagement a été daté de la fin du IIIe s. ou du début du IVe s. par la présence de céramique luisante et d'une monnaie de la seconde moitié du IIIe s. trois monnaies de la seconde moitié de ce siècle découvertes sur le dallage (2 Constance
II émis entre 346 et 361 et 1 Valentinien I émis entre 364 et 375).

48 A. Audin avait déjà repéré ce niveau de voie en 1948 lors d'un sondage réalisé à l'angle des rues Tramassac et Laurent Mourguet, ainsi qu'en 1956 (Audin 1957) à l'occasion d'un nouveau sondage rue Tramassac. 


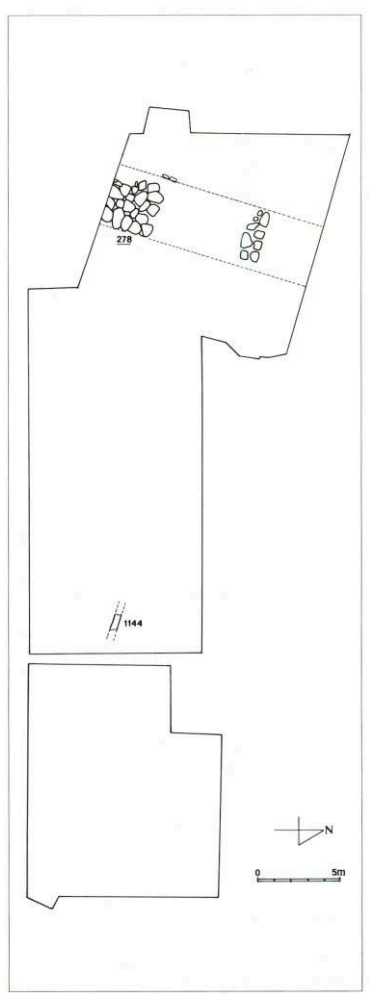

23 - Plan des vestiges de la période 3

A l'est de la voie, des couches de remplissage et d'assainissement ont été repérées. Les traces d'occupation sont très ténues : un tronçon de mur (US 1144) a été mis au jour.

A l'extrémité est de la zone fouillée, des traces d'incendie et de démolition pourraient être les témoins d'une occupation, mais aucun vestige en place n'en donne la preuve. Parmi le mobilier retrouvé, la forte proportion de céramique luisante et la présence de sigillée africaine $\mathrm{D}$ situent ces niveaux dans la deuxième moitié du IVe $\mathrm{s}$.

\section{DU IVe AU Vle SIÈCLE}

\section{LE RÉAMÉNAGEMENT DE LA VOIE (PÉRIODE 4)}

51 L'Eglise de Lyon bénéficia d'un prestige particulier dû à l'ancienneté de l'implantation chrétienne dans cette ville et au rôle actif joué par l'évêque dès la fin du IIe s. pour évangéliser la Gaule. Ce prestige se manifeste dans les réalisations architecturales de l'Eglise de Lyon. Les fouilles archéologiques menées par J.-Fr. Reynaud attestent effectivement l'importance précoce du quartier épiscopal, "un quartier urbain où se trouvait l'un des groupes épiscopaux les plus anciens et les plus prestigieux de Gaule" (Reynaud 1979). Le baptistère Saint-Etienne fut construit dès le IVe s. Une lettre de Sidoine Apollinaire, datée de 469-470, mentionne la fin de la construction de l'ecclesia.

52 A l'ouest du site de Tramassac, la voie nord/sud subit un réaménagement : une nouvelle surface (US 272) (fig. 24), exhaussée et constituée de blocs de calcaire et de galets, est installée sur un remblai. Le mobilier extrait de ce remblai (céramique estampée grise et fragments de lampes africaines de type Hayes II) assure une datation postérieure au début $\mathrm{du}$ Ve s. Cette nouvelle surface est très mal conservée. On a cependant la certitude qu'elle est orientée comme la précédente, parce qu'elle est longée par un mur 
(US 209) de même orientation. Sa fondation recoupe la frange orientale de la première voie. Sa maçonnerie, large d'un mètre, est en petit appareil très soigné ; moellons rectangulaires en assises régulières, liés avec un mortier blanc très dur. Sa largueur imposante et sa construction soignée suggèrent qu'il appartenait à un édifice important.

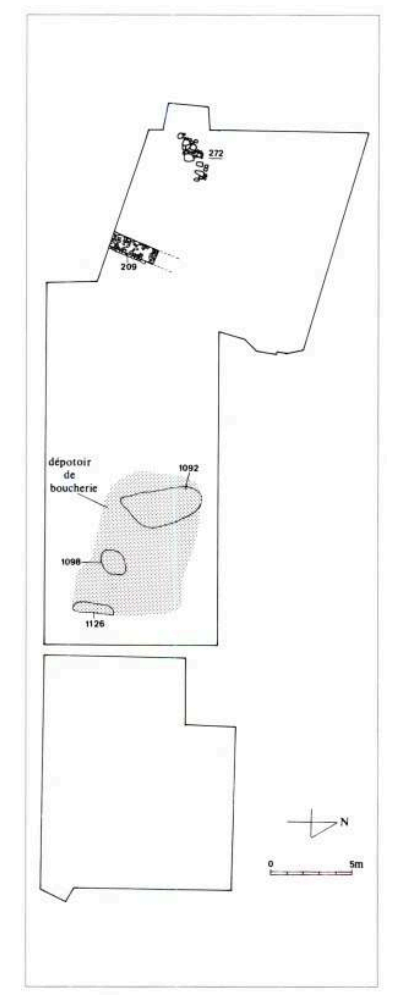

24 - Plan des vestiges de la période 4

Une série de structures contemporaines a été mise au jour lors de la fouille de la rue Carriès. Il s'agit de murs présentant des caractéristiques homogènes : construction soignée, élevée sur des fondations larges avec ressaut, en petit appareil rectangulaire lié avec un mortier en couvre-joint. Trois murs délimitent une pièce ; un autre mur, plus à l'est, n'a pas pu être raccordé à cet ensemble, mais il faut souligner qu'il s'apparente au mur 209 mis au jour dans l'îlot Tramassac. L'étude du mobilier archéologique a permis de dater la construction de l'ensemble des structures du IVe s., leur démolition de l'extrême fin du IVe s. ou du début du Ve s. Aucun indice archéologique, mobilier ou immobilier, ne permet d'expliquer la fonction de ces bâtiments. Toutefois, la proximité de la résidence épiscopale et de la cathédrale conduit à suggérer que ces bâtiments pouvaient faire partie d'un ensemble complexe. Cet espace, entre une voie imposante (le long et à l'est d'un grand axe de communication nord-sud), le groupe cathédral et les thermes, semble bien être utilisé à la même époque pour compléter l'équipement monumental du complexe épiscopal, lequel comportait à la fois "églises, évêché, réfectoires, dispensaire, hospice" (Duchesne 1902, p. 399 ; Lesne 1910, p. 197 ; Villedieu 1990, p. 112)

Dans la partie est de l'îlot Tramassac, des remblais contenant des matériaux de construction (briques, tuiles, mortier) et un sol en terre battue, attestent une occupation. Par contre, aucune structure contemporaine n'a été découverte. Le mobilier céramique présent dans ces couches (céramique estampée grise et bords à 
bandeau étroit en céramique commune sombre) suggère une datation dans la deuxième moitié du Ve s.

Au centre du site, un espace sert de champ d'épandage à des bouchers ou des équarisseurs. Un dépotoir contenant une importante quantité d'os d'animaux a en effet été mis au jour. Il est daté de la fin du Ve s. par l'analyse du matériel céramique (forte proportion de céramiques luisantes, céramique estampée grise en plus grande quantité). L'analyse des restes osseux a permis de caractériser ce dépotoir de boucherie : $98 \%$ des restes ont été attribués à des mammifères domestiques. Le rejet de ces restes de boucherie est organisé en grand épandage, dont la nature particulière tient à leur importance, car ils réunissent dans certains cas plusieurs milliers de restes. Le rythme de dépôt semble assez rapide. Il s'agit dans certains cas d'entassements d'os où le bœuf est prédominant. Il faut évidemment écarter l'hypothèse d'un dépôt lié à un seul habitat, il pourrait en revanche correspondre à la consommation d'une communauté entourant l'évêque.

\section{L'HABITAT DU VIe SIÈCLE (PÉRIODE 5)}

$56 \mathrm{Au}$ VIe s., l'ensemble du terrain est nivelé avec un apport de remblais qui scelle le dépotoir de boucherie. Sur les remblais, s'installe un habitat dont il subsiste à l'est, un mur conservé sur toute sa longueur et des fragments de murs au sud et au nord ; le mur ouest a pour sa part entièrement disparu (fig. 25). Ces murs présentent un appareil irrégulier composé de moellons de calcaire, de blocs de schistes et de gros galets liés entre eux par un mortier sableux très altéré (fig. 26, 27).

Le sol (US 1095) extérieur au bâtiment contient de nombreux éclats de pierre et de mortier blanc; il correspond à un niveau de travail. Quant au sol d'occupation intérieur (US 1096), il semble avoir été confectionné en mortier blanc, comme l'attestent de nombreux lambeaux de mortier très décomposé. Le foyer 1099, contemporain de ce sol, se caractérise par une couche cendreuse bordée de terre rubéfiée.

Le mobilier retrouvé dans le sol de travail et dans la tranchée de fondation du mur nord montre une augmentation des céramiques communes grises par rapport aux périodes précédentes. Cette observation et la position stratigraphique de l'habitat (postérieure au dépotoir de boucherie) permettent de le dater du VIe s.

Dans la partie ouest du chantier, une autre structure a été mise au jour. Les traces en sont relativement ténues car il ne reste qu'une seule assise de fondation de deux murs. Par contre, la présence d'un sol et les traces de foyer à l'intérieur laissent penser qu'il s'agit d'un habitat.

60 Entre ces deux structures d'habitat, des tronçons de murs (US 1122, 1123) ont été découverts. La fouille n'a pas permis de mettre en évidence les sols correspondants ; leur aspect (gros blocs de calcaire liés au mortier) empêche de les intégrer à la structure décrite précédemment et leur contemporanéité reste une hypothèse.

61 Un dallage en blocs de calcaire (US 231), dénotant un nouveau niveau de voie, recouvre celui de la période 3. Il est aménagé avec un apport volontaire de remblais créant une surélévation de l'axe de circulation. Ce remblai a livré le même type de mobilier osseux que le dépotoir de boucherie, d'où il semble donc avoir été extrait. 


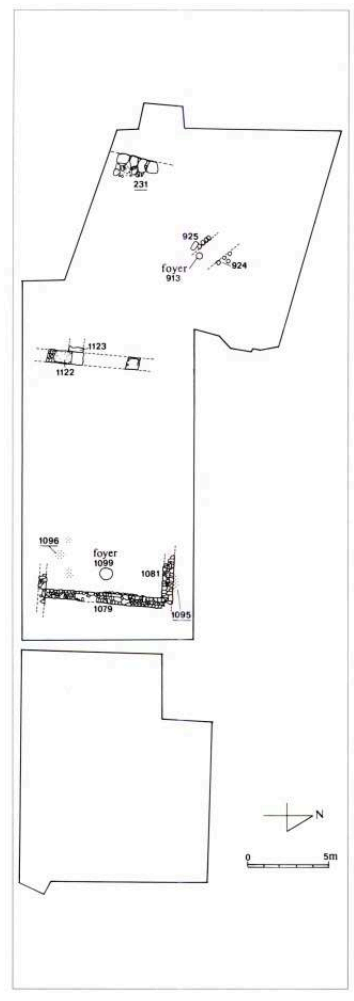

25 - Plan des vestiges de la période 5

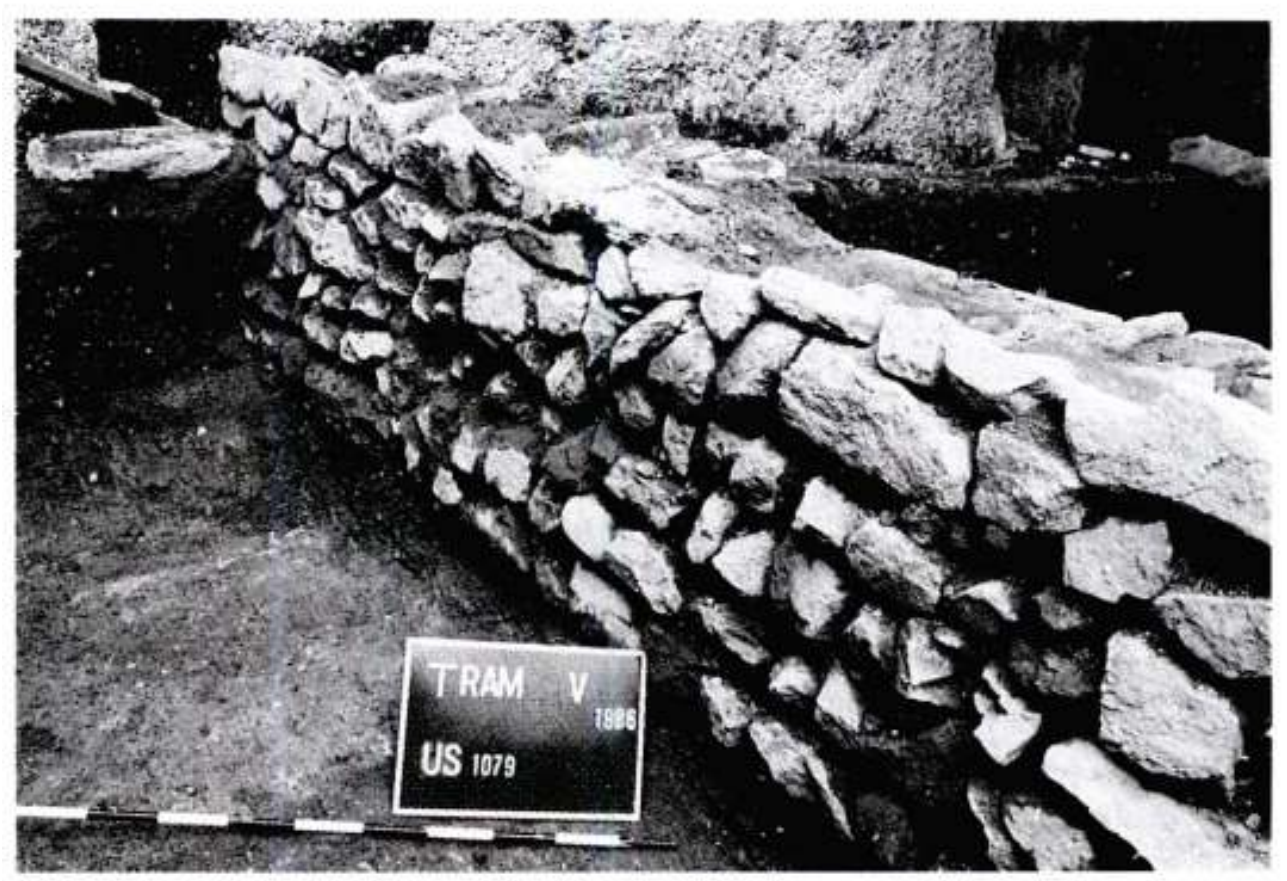

26 - Détail du mur est (US 1079) 


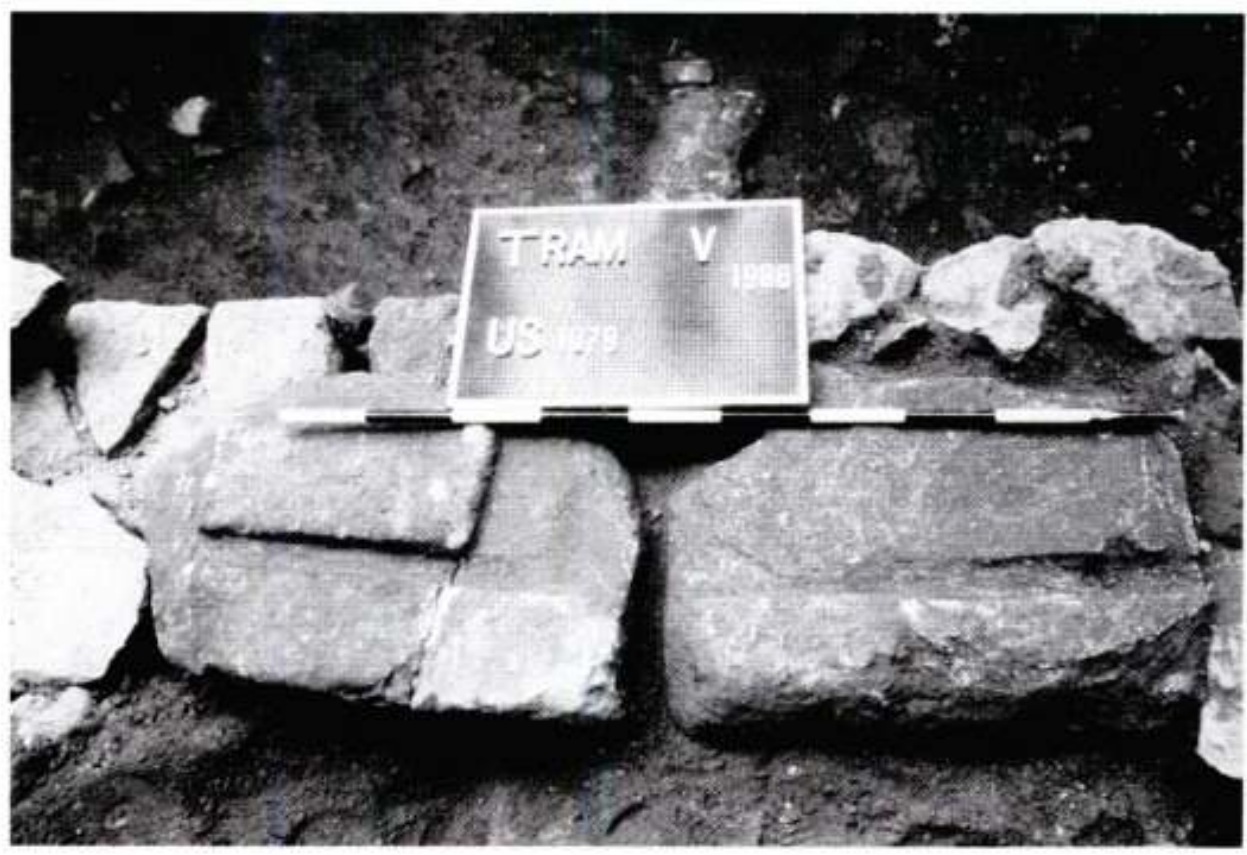

27 - Détail du seuil (US 1079)

62 L'aménagement de cette voie est ainsi contemporain ou antérieur à la construction de la structure d'habitat qui scelle le dépotoir de boucherie, soit du VIe s.

\section{UN ABANDON PARTIEL DU SITE À LA FIN DU VIe SIÈCLE (PÉRIODE}

6)

63 Sur l'ensemble de l'espace fouillé, une grande quantité de couches témoigne de l'abandon et de la destruction des structures bâties. Ces niveaux contiennent de nombreux débris d'éléments de construction, tels que des fragments de tuiles, de briques, de blocs taillés.

Dans la zone est, certaines couches présentent d'importantes marques de rubéfaction, témoins d'un incendie. Quant au matériel recueilli, le pourcentage élevé de céramique grise et la présence de bords à bandeaux placent cette démolition au seuil du Haut Moyen Age.

65 A l'ouest, la voie (US 231) continue en revanche à être utilisée telle quelle, jusqu'au IXe s.

\section{LES VESTIGES D'UN BÂTIMENT DE LA FIN DU VIe SIÈCLE (PÉRIODE 7)}

66 La période 7 correspond à un réaménagement du secteur avec une réorganisation complète de l'espace, à l'exception de la voie, maintenue en l'état.

67 Sur les couches de démolition de la période 6, qui recouvrent une grande partie de l'espace fouillé, apparaissent de nouveaux vestiges de constructions avec, dans la partie est du site, une imposante maçonnerie (US 620), des couches d'occupation et, au centre, un sol en mortier de tuileau (US 1053) conservé sur une surface importante (fig. 28). 
La maçonnerie (US 620), axée nord/sud, présente dans ses fondations des éléments de réemploi (dalle de calcaire, chapiteau à feuille d'acanthe); les dernières assises sous le ressaut de fondation sont bien appareillées et montrent un parement régulier semblable à celui de l'élévation. Ce mur profondément ancré dans les couches antérieures semble avoir eu une fonction de soutènement de terrasse.

$\mathrm{Au}$ centre, le sol en mortier de tuileau très compact (US 1053), d'une épaisseur variant entre 5 et $6 \mathrm{~cm}$, repose sur un radier composé de galets, de pierres posées sur chant ou de blocs de calcaire (fig. 29). Bien qu'il ait été mis au jour sur une surface d'environ 10 $\mathrm{m}^{2}$, il n'a pas été possible, compte tenu des contraintes de la fouille, de vérifier sur le terrain sa contemporanéité avec certaines des maçonneries.

70 Cependant ces deux structures (mur et sol), séparées de seulement $2 \mathrm{~m}$ (hiatus dans la fouille dû à la mise en place des parois moulées) et installées dans les mêmes niveaux, conduisent à supposer leur appartenance à un unique bâtiment.

71 L'examen du mobilier céramique de cette période ne permet pas d'avancer de datations précises. Seule la position stratigraphique du sol (US 1053) situe la construction de ces structures à la fin du VIe s.

72 L'analyse numismatique n'apporte pas d'éléments déterminants pour les datations, car les monnaies retrouvées dans ces niveaux sont du IIIe et du IVe s.; cette présence de matériel résiduel antique se retrouve jusque dans les niveaux du XIXe s.

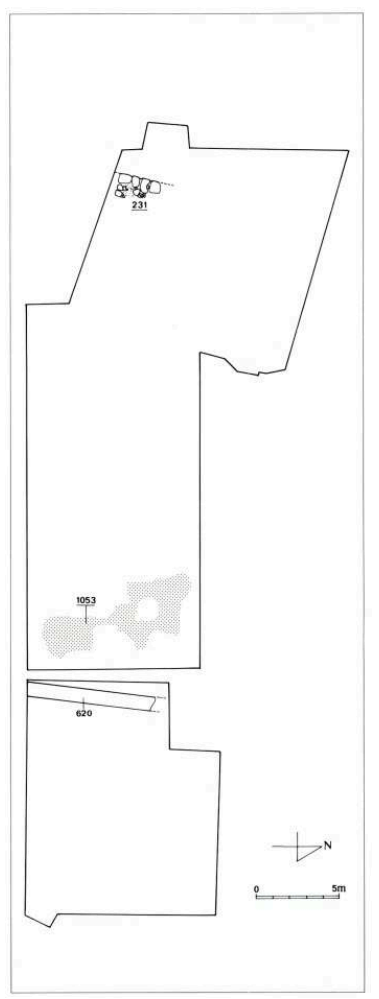

28 - Plan des vestiges de la période 7 


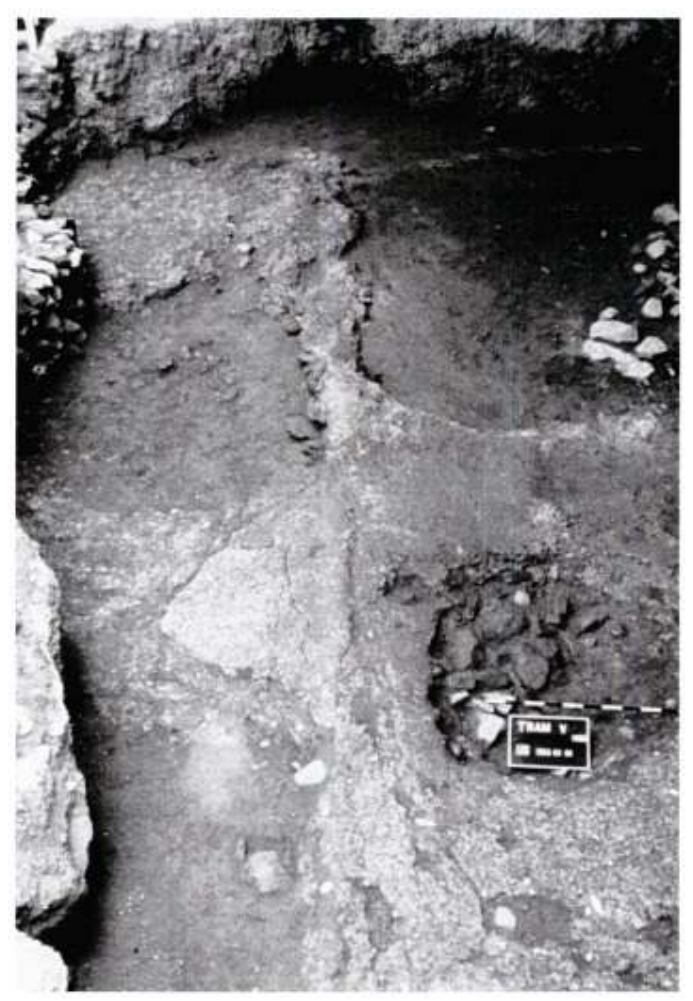

29 - Sol en mortier de tuileau (US 1053)

73 Les structures des périodes 3 à 7 dénotent donc la mise en place progressive d'un quartier urbain de l'Antiquité tardive, à proximité immédiate du groupe épiscopal. L'espace est structuré par une voie dallée nord/sud, au pied de la colline, qui, installée au tournant du IIIe et du IVe s., continuera à fonctionner jusqu'à l'époque moderne (avec un glissement progressif vers l'ouest).

74 En l'absence de pont sur la Saône à la hauteur du quartier épiscopal, cette voie fut certainement l'axe de circulation principal du quartier, permettant de rejoindre des axes transversaux situés en amont et en aval (Reynaud 1990, p. 226). Le quartier bordé par la voie comprenait, entre autres, des bâtiments de service associés au groupe épiscopal. La fouille de l'avenue A. Max avait ainsi permis de repérer des thermes publics de cette époque. La nouvelle fouille a mis en évidence des vestiges de maçonnerie appartenant sans doute en bonne partie à des édifices importants, mais que l'on ne peut pas identifier précisément en raison de leur caractère très lacunaire.

La trame urbaine paraît encore lâche au Ve s., puisqu'un espace vaste, libre, sert de dépotoir de boucherie, tandis qu'elle se resserre au siècle suivant.

Le glissement vers le Haut Moyen Age s'amorce franchement à la période suivante, avec la disparition des modes de construction de type romain et l'apparition de bâtiments beaucoup plus précaires.

\section{DU VIle AU XIe SIÈCLE}

77 La lecture archéologique de l'îlot Tramassac présente les difficultés inhérentes aux sites urbains à stratification complexe : si la présence antique est nettement perceptible par ses structures et identifiable par son mobilier et si la même remarque peut être faite 
pour l'époque postérieure au XIIe s., les vestiges de l'époque intermédiaire, du VIIe au XIe s., restent par contre bien plus difficiles à caractériser. La méconnaissance due à une carence à la fois des archives écrites et des archives du sol, ainsi que l'absence d'éléments de comparaison, entraînent de grosses difficultés d'interprétation (périodes 8 à 13).

Bien que la céramique commune antique ne soit pas toujours bien connue, celle d'importation constitue un traceur chronologique très appréciable. Pour le Haut Moyen Age, en revanche, celle-ci disparait. L'évolution de la culture matérielle est encore mal définie dans son ensemble par manque d'éléments comparatifs et reste donc difficile à cerner dans nos régions. Qui plus est, les couches du Haut Moyen Age contiennent toujours en quantité notable du mobilier résiduel plus ancien. A ce problème, il faut également ajouter celui de la stratification du site pour ces périodes où la lecture verticale du terrain est rendue parfois impossible, ne révélant aucune différence de structure, de texture et de couleur des couches. Face à ces deux problèmes majeurs, le travail comparatif de céramologie et d'analyse stratigraphique a été d'autant plus nécessaire et enrichissant pour percevoir les périodes d'occupation et apporter des datations.

79 Le passage de l'Antiquité tardive au Haut Moyen Age, bien que progressif, est néanmoins manifeste et se révèle sur le site à travers divers éléments. Parallèlement aux structures pré-existantes qui continuent à être utilisées, comme par exemple le bâtiment de la période précédente, ainsi que l'axe de circulation, apparaît un nouveau type de construction aux techniques plus rudimentaires. Les maçonneries, qui étaient jusqu'alors liées au mortier, et dont les moellons taillés formaient des assises relativement régulières, sont désormais remplacées par des murs en pierres grossièrement équarries, liées à la terre (fig. 30). Les bâtiments, de petites dimensions, correspondent à des habitats plus précaires avec des sols en terre battue et des foyers aménagés à même le sol. Ce nouveau type d'architecture en pierres liées à la terre s'observe du VIIe au XIe s.

Une succession de phases d'habitat est concentrée dans la partie ouest, alors que sur le reste de la zone fouillée, seules quelques traces témoignent d'une occupation. Les vestiges mis au jour montrent une absence d'évolution dans les techniques de construction pendant toute la période.

81 Les matériaux de construction utilisés sont variés : les bases des murs sont constituées d'un mélange de galets du Rhône, de blocs de calcaire bruts ou grossièrement équarris, de blocs de schiste ou de granit, le tout de module variable. Les constructions présentent un appareil irrégulier, dont le parement est sommairement dressé, formé de blocs posés en laissant des interstices remplis par de la terre. Certaines structures, telle l'US 195, possèdent également un appareil hétéroclite où alternent des assises irrégulières et un appareil en arêtes de poisson.

Les maçonneries ne sont conservées que sur deux ou trois assises, ce qui ne permet pas de projeter leur hauteur et encore moins l'aspect de la toiture. Il en est de même des matériaux de l'élévation, pour laquelle l'hypothèse la plus plausible reste l'élévation en pierre et en terre, sans que l'on puisse préciser leur mode de mise en volume; ce sont les seuls matériaux retrouvés dans les démolitions. 


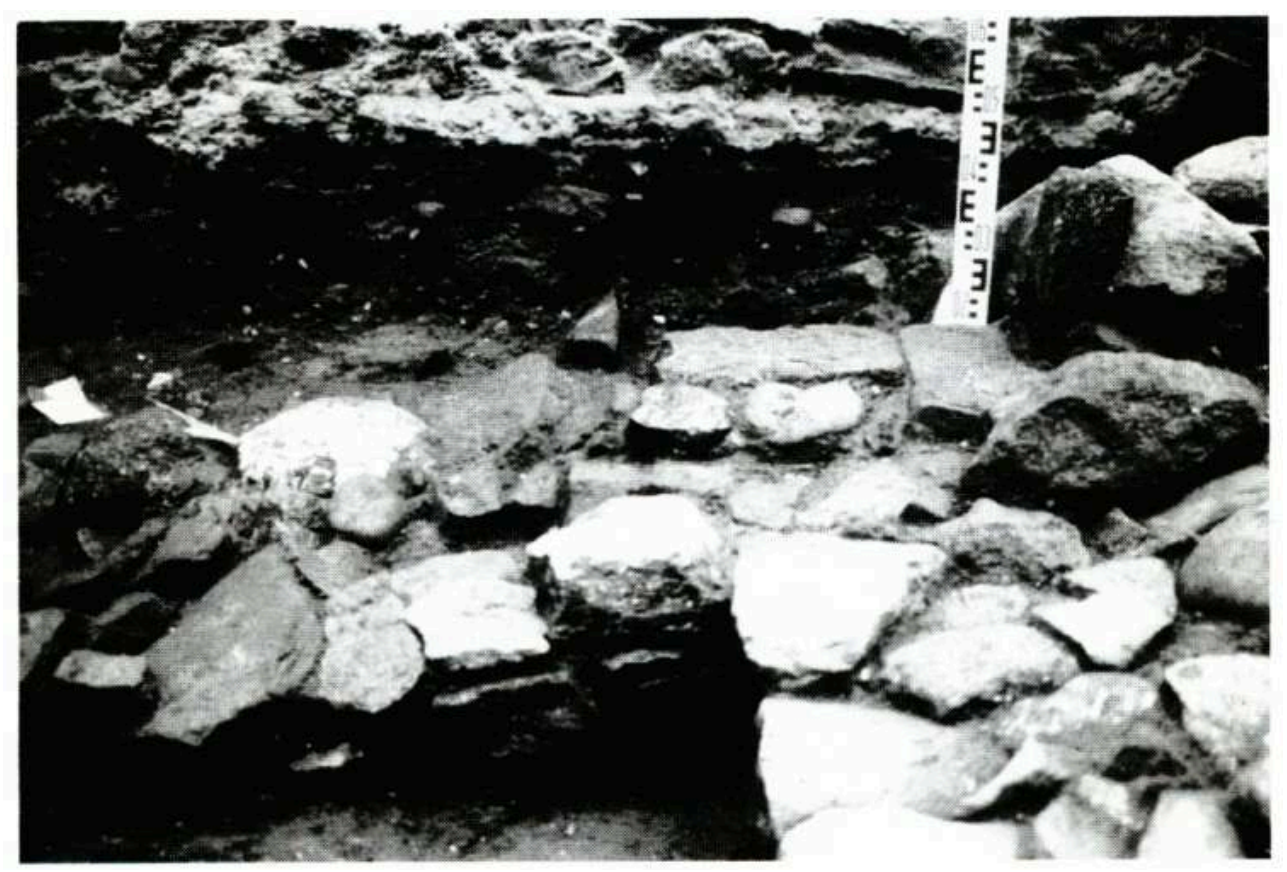

30 - Détails des techniques et des matériaux de construction

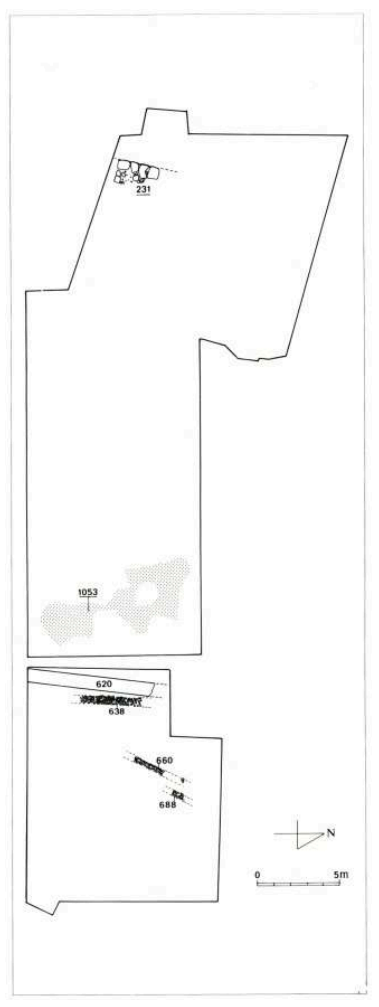

31 - Plan des vestiges de la période 8

Les vestiges d'habitat, mal conservés, parfois en limite de fouille, empêchent de connaître les dimensions des bâtiments. Seules deux des structures (périodes 12 et 13) sont conservées sur trois côtés et, dans l'hypothèse d'un habitat d'une seule pièce carrée, la surface est inférieure à $25 \mathrm{~m}^{2}$ pour la période 12 et à $20 \mathrm{~m}^{2}$ pour la période 13 .

84 La fonction de ces bâtiments est surtout attestée par les foyers aménagés directement sur le sol, parfois construits en matériaux de récupération (tuiles, briques) et délimités 
par des blocs de calcaire, mais le plus souvent la fouille a seulement mis au jour des aires mal délimitées de terre rubéfiée recouverte de cendres et de charbon de bois. Hormis les foyers, aucun élément du confort (puits, dépotoir...) n'a été découvert.

\section{L'HABITAT MÉROVINGIEN (PÉRIODES 8 À 10)}

Durant les périodes 8 et 9, la voie (US 231) créée au VIe s. est toujours utilisée. Dans la partie est, trois fragments de murs ont été mis au jour. Deux d'entre eux sont parallèles et très rapprochés; ils sont construits en moellons et en galets liés à la terre, tout comme le mur (US 638) un peu plus éloigné et orienté différemment des deux premiers. Le bâtiment de la période 7 (mur 620 et sol 1053) semble encore utilisé (fig. 31). Le mobilier peu abondant ne permet pas d'apporter une datation précise. Le pourcentage de céramique gallo-romaine, malgré une baisse sensible, reste encore très élevé, entre 65 à $69 \%$. Cependant la fragmentation de la céramique prouve que les niveaux en question ont subi un important brassage; toutefois un nouveau type de céramique est utilisé avec des formes attribuables au Haut Moyen Age.

Durant la période 10 qui couvre les VIIe et VIIIe s., une grande partie du site semble abandonnée, sauf à l'ouest où les vestiges d'un mur (US 257-258) et d'un foyer (US 259) témoignent d'une occupation. Ce mur est construit en bordure de la voie (US 231) qui a été réaménagée dans sa partie est ; elle mesure alors 2,50 m de large (fig. 32).

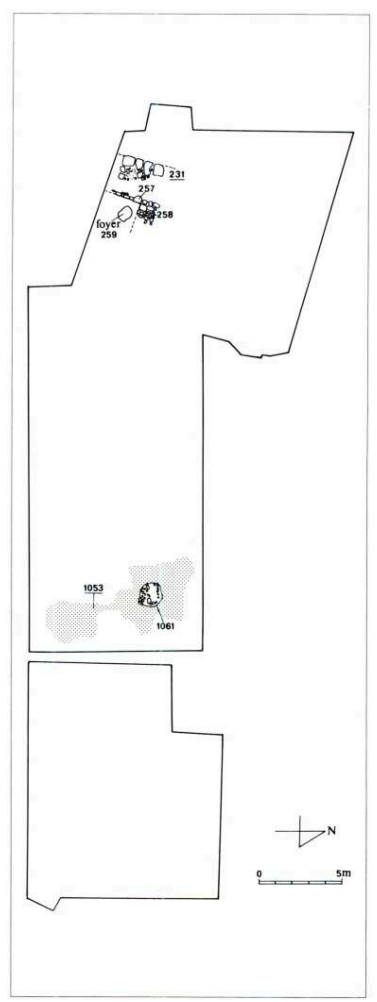

32 - Plan des vestiges de la période 9

Le mobilier céramique conserve une part importante de matériel antique (entre 62 et $77 \%$ ) ; le reste du matériel, bien que mal connu, a pu être identifié par comparaison avec celui des fouilles de Saint-Laurent-de-Choulans (Reynaud 1981). 
$\mathrm{Au}$ centre du terrain, l'utilisation du bâtiment des périodes précédentes paraît difficile à préciser. Bien qu'aucune trace n'atteste son occupation, il est fort probable qu'il ait été utilisé jusqu'au IXe s., époque à laquelle il est détruit et percé par des fosses.

\section{L'HABITAT CAROLINGIEN (PÉRIODES 11 ET 12)}

Une nouvelle structure d'habitat est construite dans la zone ouest (période 11) (fig. 33). Les fondations et la base des élévations des murs sud et est sont conservées. Deux niveaux de sol en terre battue, qui attestent l'occupation de cet habitat, ont été dégagés. Au sud de cette structure, un ensemble de dalles de calcaire jointives peut être interprété comme un sol de cour. Il est installé sur l'ancien axe de circulation. Pour les époques postérieures, nous savons que cet axe existe toujours, puisqu'il s'agit de la future rue Tramassac, mais décalé plus à l'ouest, au pied de la colline. Son déplacement pourrait donc s'effectuer dès cette époque.

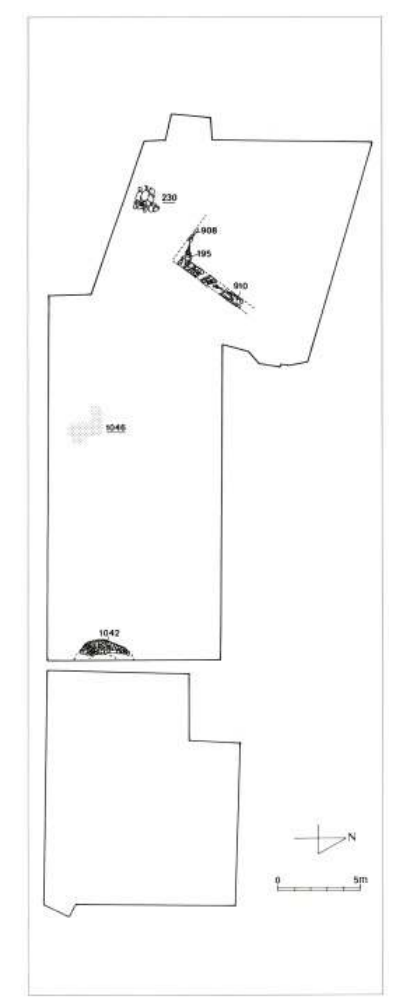

33 - Plan des vestiges de la période 11

$90 \mathrm{Au}$ centre du terrain, un fragment de sol en mortier blanc sur hérisson n'a malheureusement pas pu être interprété, de même qu'un élément maçonné situé en limite de fouille à l'est (Tramassac V) et pris dans les parois moulées du chantier. De forme arrondie, ce dernier pourrait être le parement externe d'une structure enterrée comme une glacière ou un dépotoir.

91 Le mobilier céramique est encore pour une bonne part de type antique (jusqu'à $1 / 3$ dans certains lots), mais des formes typiques attribuées aux IXe et Xe s. apparaissent également. Bien qu'aucun vase n'ait pu être reconstitué en entier, le mobilier étant très fragmenté, les éléments identifiables sont suffisamment nombreux pour établir une chronologie relative entre les différentes étapes de cette période. 
Durant la période 12 (fig. 34, 35), un réaménagement est réalisé dans la zone ouest du terrain. Une nouvelle construction est bâtie sur la précédente. L'espace occupé par cet habitat semble étendu, bien que la construction d'un mur durant la période 14 empêche de préciser son extension vers le nord (US 892-895).

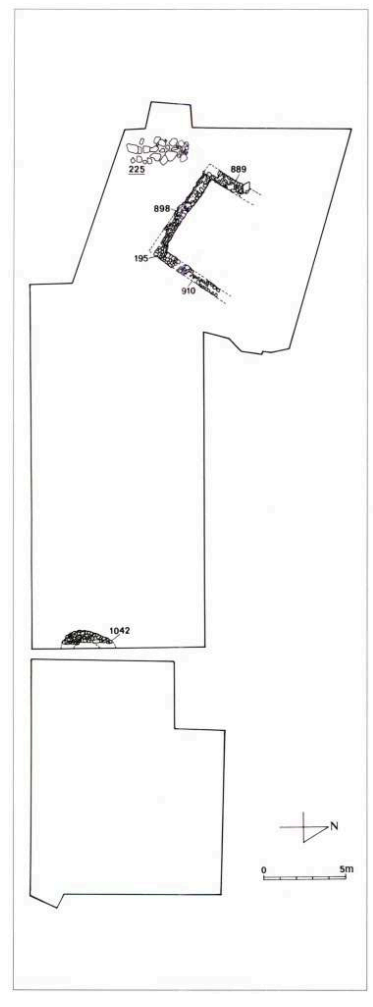

34 - Plan des vestiges de la période 12

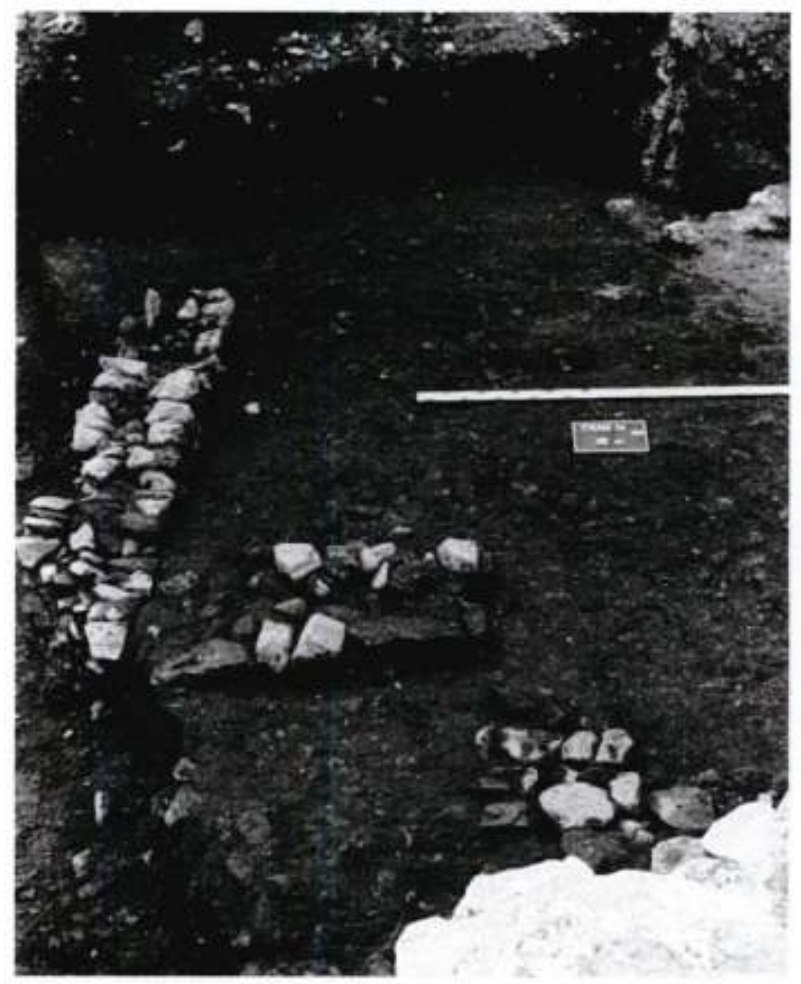

35 - Murs (US 898 et 910) 
Ces travaux provoquent une légère surélévation du terrain, qui entraîne du même coup l'aménagement d'un nouveau sol en dalles de calcaire (US 225) sur le précédent (US 230). Le mobilier reste semblable à celui de la période 11.

\section{UNE NOUVELLE STRUCTURE D'HABITAT AU XIe S. (PÉRIODE 13)}

94 Preuve de la précarité de ce type de construction, la zone ouest est à nouveau réaménagée et une nouvelle habitation est construite, toujours en pierres liées à la terre, suivant une orientation différente; elle est en partie conservée sur trois côtés. A l'intérieur du bâtiment, un foyer à même le sol en terre battue a été mis au jour (fig. $36,37)$.

A ce stade apparaît un nouveau type de céramique, caractérisé par sa glaçure parsemée et peu couvrante, déjà attesté à Lyon sur le site de Saint-Just dans les couches postcarolingiennes (Reynaud 1981); des fonds marqués et des formes typiques de l'époque antérieure continuent à exister. Cette caractéristique permet de situer cette période aux XIe et XIIe s.

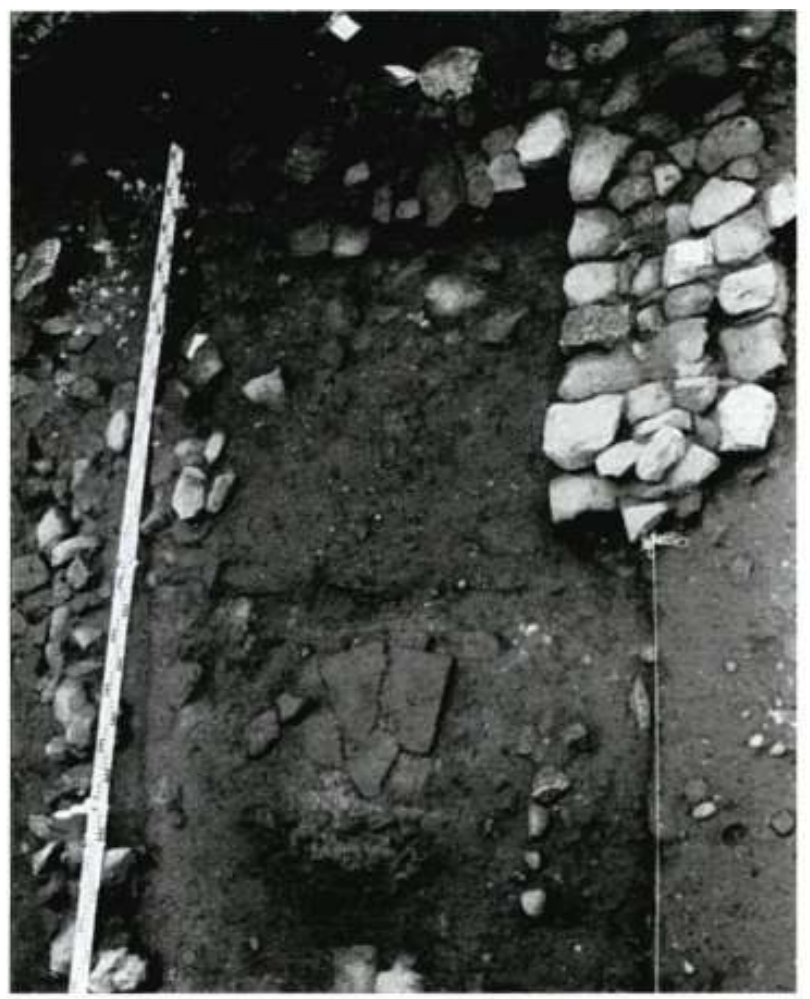

36 - Foyer (US 217) en cours de fouilles

Dans la partie est du terrain, où aucun niveau d'occupation postérieur au VIIe s. (période 9) n'a été décelé par la fouille, une construction rectangulaire de dimensions réduites $(2 \times 4,5 \mathrm{~m})$ a été mise au jour (fig. 38). Construite en pleine terre et perçant les niveaux gallo-romains, cette dernière, bâtie en moellons de calcaire liés à la terre, présente un parement uniquement à l'intérieur. L'absence de mobilier et la texture des couches de comblement ne permettent pas de déterminer avec certitude sa fonction (dépotoir, fosse d'aisance ?). 
97 Le quartier sud-ouest du complexe épiscopal est donc habité en permanence du début du VIIe s. à la fin du XIe s. C'est également une époque où il n'y a pas de programme monumental dans ce secteur. La destination précise des bâtiments du Haut Moyen Age mis au jour n'apparaît pas clairement. La présence de foyers et d'un mobilier céramique abondant montre leur utilisation à des fins domestiques, mais le caractère rudimentaire des modes de construction et la faible superficie des "unités d'habitation" peut faire penser à des abris précaires et provisoires, liés, par exemple, à des chantiers de construction et édifices monumentaux. Si tel n'était pas le cas, les vestiges d'habitat de l'îlot Tramassac, situés à proximité de la cité épiscopale, montreraient le contraste très fort existant entre bâtiments publics (religieux) et habitat civil aux époques mérovingienne et carolingienne. On perçoit, en effet, une absence complète de volonté d'urbanisme et un tissu très lâche de maisons rudimentaires.

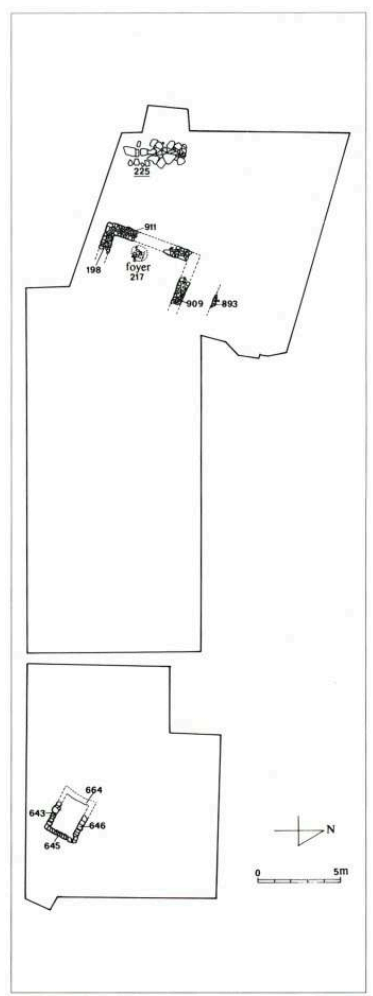

37 - Plan des vestiges de la période 13 


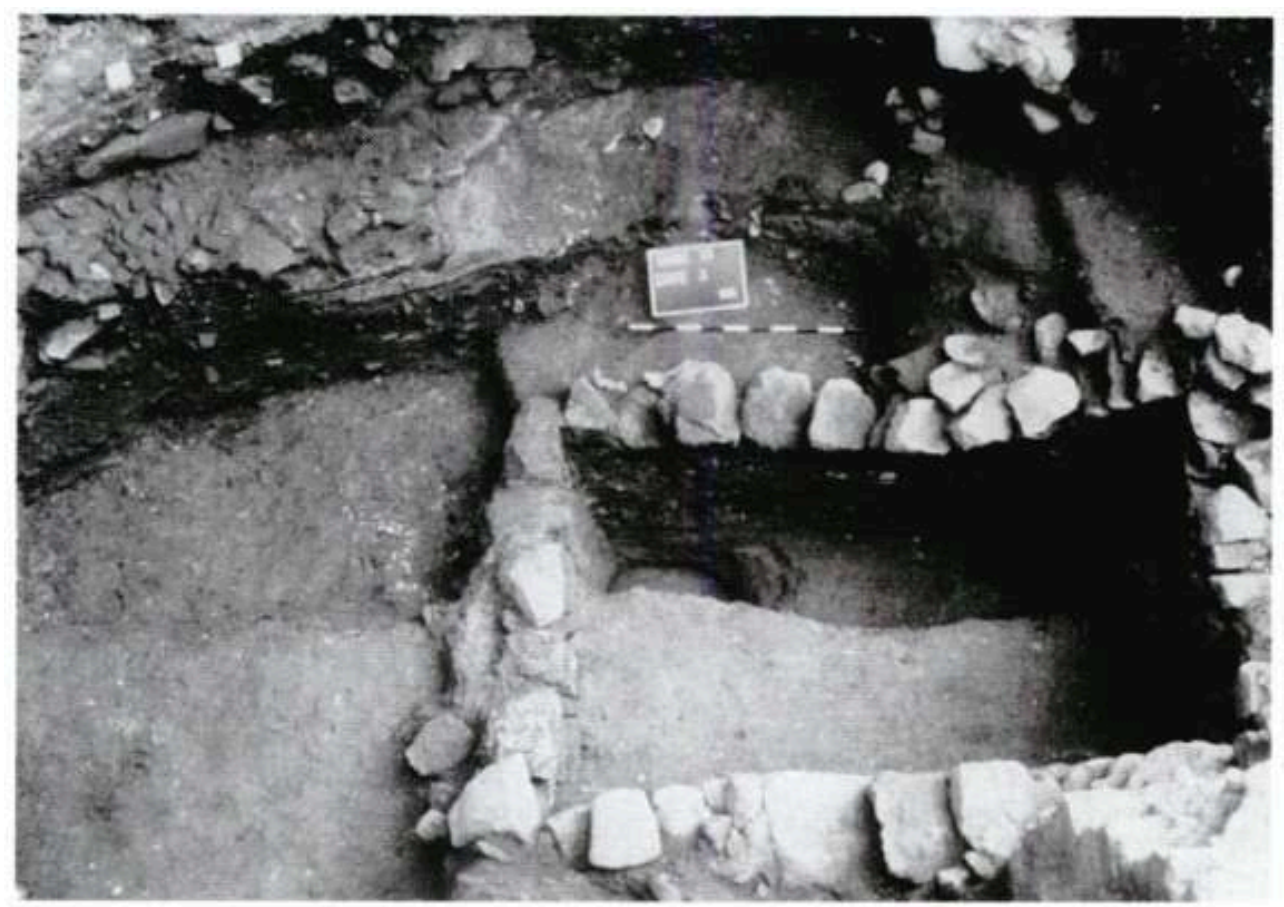

38 - Structure rectangulaire

\section{DU XIIe AU XVIIle SIÈCLE}

Si les populations habitant ce quartier et leurs activités sont actuellement peu ou mal connues pour le Haut Moyen Age, la documentation textuelle permet de s'en faire une meilleure idée à partir du XIIe s.

Deux groupes se côtoient, le monde ecclésiastique et le monde laïc séparés par le mur de l'enceinte canoniale, construit entre 1165 et 1180 . De fait, à partir du XIIe s., l'occupation du site va subir de nombreuses transformations qui se manifestent sous plusieurs aspects. Une part importante de la problématique de la fouille était de déterminer où se situait la muraille de l'enceinte canoniale dans l'îlot Tramassac, secteur dans lequel son tracé reste le plus hypothétique, et de mettre en évidence les caractères archéologiques qui puissent traduire la différence de statut entre habitat canonial et habitat laïc.

100 Le tènement situé dans la partie est de la fouille est en effet situé dans l'enceinte canoniale de Saint-Jean. C'est justement dans ce secteur qu'ont été mises au jour les traces d'occupation certainement les plus monumentales du site; elles peuvent être attribuées à l'hôtel du Doyenné connu par des textes et des plans anciens.

101 Parallèlement, à l'ouest, se développe un habitat laïc qui connaît la même croissance que le quartier avoisinant. Il convient de souligner que la limite occidentale de cet habitat est la rue Tramassac, qui conserve l'axe de la voie existant depuis l'Antiquité. 


\section{PÉRIODE 14}

\section{L'HABITAT EXTRA-MUROS}

102 trois côtés, celui-ci présente des maçonneries liées au mortier. Un seuil (US 150) en place dans le mur ouest (US 173) marque l'emplacement d'une porte donnant accès directement à la rue Tramassac, pavée de galets disposés en "tête de chat" (US 269) (fig. 39,40).

de cet habitat, une imposante maçonnerie (US 892/895) orientée est-ouest est construite en moellons de calcaire liés au mortier. Ce mur perfore des structures du Haut Moyen Age et prend assise sur le premier niveau de la voie antique (US 278). L'absence de relation avec d'autres structures ne permet pas de proposer une interprétation. On peut toutefois évoquer l'hypothèse d'un mur marquant une limite de propriété.

104 A partir de cette époque, toutes les constructions (ou réaménagements) adopteront une forme rectangulaire et demeureront conditionnées par l'existence de Taxe de circulation. Ainsi, dès le XIIe s., le parcellaire se constitue, résultat progressif du morcellement des tènements. Une nuance doit être faite entre "bâti" et "parcelle", car dans un premier temps, seul le bâti est discernable; les limites de la parcelle, ou du moins les témoins de leur matérialisation, ont pu disparaître; ils ne sont ni décrits, ni même mentionnés dans les textes. Le Plan scénographique de la ville de Lyon donne en revanche des exemples de clôtures: à l'extérieur du noyau urbain, plusieurs types coexistent (palissades en bois diverses, murailles...) alors que dans la ville, seuls de hauts murs sont représentés.

Le mobilier céramique retrouvé dans le niveau de rue, contemporain de cet habitat, ainsi que le type de revêtement en "tête de chat", permettent de dater ces aménagements du XIIe s. Quant à l'étude numismatique, elle n'a apporté aucun complément, l'ensemble des monnaies issues des couches de cette période étant résiduel ou illisible.

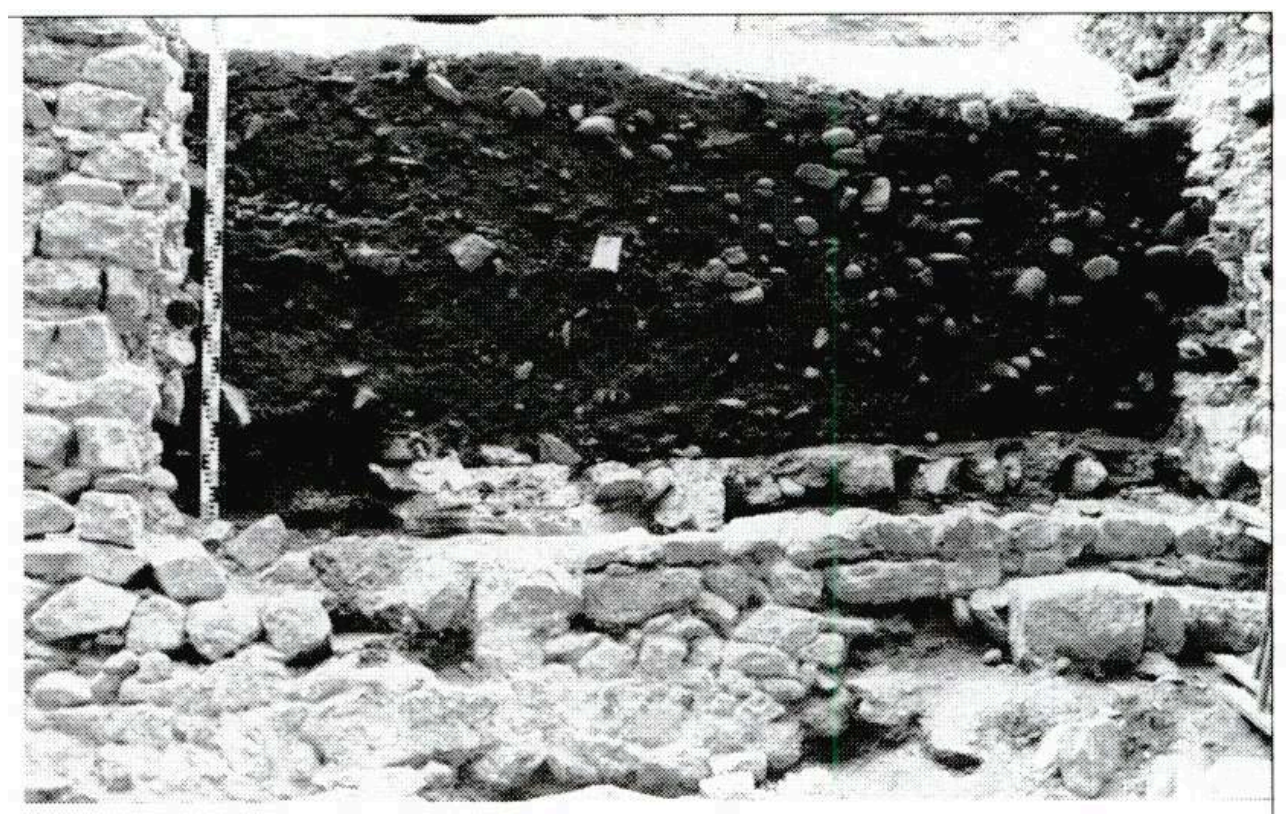

39 - Seuil (US 150) et niveau de rue (US 269) 


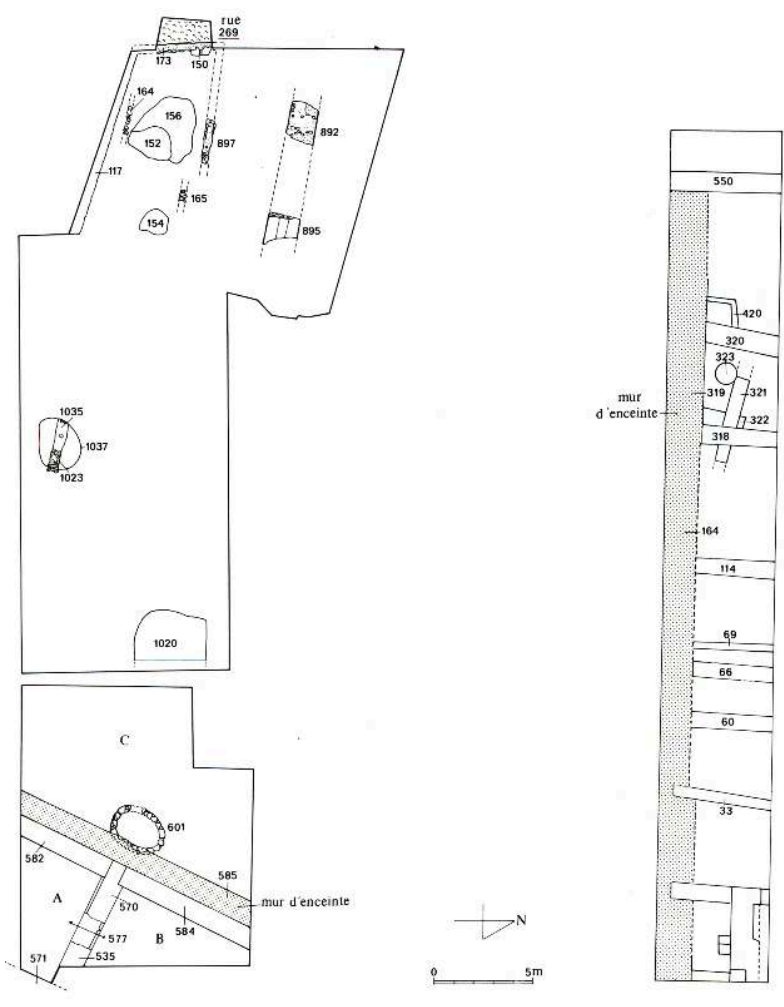

40 - Plan des vestiges de la période 14 (Tramassac et Carriès)

Entre le bâtiment et le mur d'enceinte continue à exister un vaste espace vide que l'on peut proposer d'identifier à des jardins contigus à l'hôtel du Doyenné, mentionné en 1419 (A. D. R. 10. G. 84. folio 33). Les seules structures repérées dans cet espace sont un puits (US 601 616) et une fosse-dépotoir (US 1020) (fig. 40).

107 Le puits de section ovale mesure 2,30 m sur son axe le plus large et 1,40 m sur le plus étroit; il est construit en gros blocs de calcaire bien appareillés. Arasé dans la partie supérieure, il est cependant conservé sur environ $4 \mathrm{~m}$ de profondeur. Peu de matériel identifiable a été sorti de la couche inférieure du puits; cependant la présence de la moitié d'un cruchon en pâte rouge, décoré de bandes de barbotine moletées, permet de proposer une datation assez précise, soit la première moitié du XIIIe $\mathrm{s}$. Cette forme est comparable au type $2 \mathrm{~A}$ du mobilier retrouvé à Anse (au nord de Lyon) dans un silo du château des Tours (Vicard 1985). Elle présente le seul élément déterminant pour la chronologie de la construction du puits qui doit forcément être antérieure à cette époque ou contemporaine.

Le dépotoir est installé dans une fosse de forme rectangulaire; il contient une grande quantité de mobilier céramique qui correspond en majorité à de la vaisselle de qualité. Il a pu être daté de la fin du XIIIe s. et du début du XIVe s. Lui aussi est à rapprocher par ses formes du matériel céramique retrouvé à Anse dans les deux dépotoirs du château, avec cependant l'apparition de formes et décors nouveaux. A également été isolé au sein de cet ensemble un fragment de céramique du Proche-Orient.

Parmi les fragments de verre, plusieurs formes ont été identifiées :

- trois fragments appartiennent à des verres à tige creuse de type A (selon Foy 1989) (fig. 41) qui font leur apparition à la fin du XIIIe s. Le fragment de panse présente un décor de fines côtes lissées à chaud ; la tige et le pied (en forme de disque) sont décorés de côtes moulées en relief qui se terminent sur le pied en un mouvement circulaire. 
- deux goulots de gourde de type $\mathrm{F}$, vert pâle, très altérés, ont un décor de cordon pincé sur le haut de la panse appliqué après soufflage.

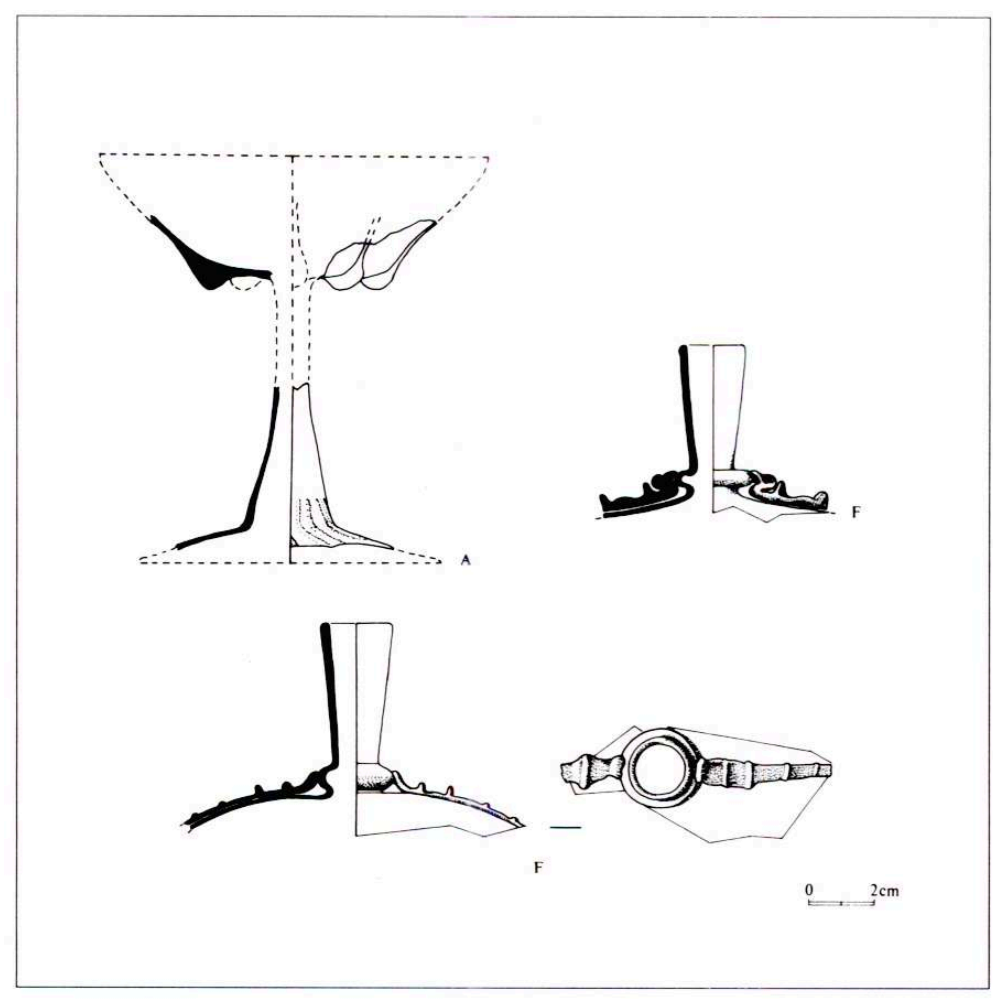

41 - Verre à tige $(A)$ et goulots de gourde $(F)$

110 Situé entre les deux zones d'habitat, cet espace est difficile à interpréter. La fouille a prouvé qu'il est vierge de constructions depuis le VIe s. Le sol de tuileau construit au VIe s. a dû être réutilisé un certains temps avant d'être abandonné et recouvert par l'humus. La présence de quelques tessons de céramique qui se complètent, alors qu'ils proviennent de couches différentes, pourrait aussi s'expliquer par le brassage de la terre d'un jardin dans lequel des arbres ont été plantés; les textes mentionnent ces plantations. Il est donc plausible de penser que cette description s'applique à cet espace. La découverte dans le dépotoir, de céramiques de luxe montre que, "terrain vague" ou jardin, les chanoines s'étaient déjà approprié cet espace situé hors de l'enceinte, ce qu'atteste l'étude des archives. Précisément à la même époque, l'hôtel est détenu non pas par un doyen, mais par un évêque (fig. 42). Guillaume de Thurey, doyen entre 1340 et 1356, conserve en effet après son accession à l'épiscopat et jusqu'à sa mort en 1365, l'usage de la maison qu'il avait acquise pendant son décanat (1343. A.D.R. 10.G. 666), sans la céder à son successeur dans la fonction de doyen lors de sa résignation de charge; son neveu Philippe de Thurey aurait ensuite repris cette maison et l'aurait occupée durant son propre épiscopat entre 1389 et 1415. Les mentions d'actes capitulaires sont insistantes sur ce point: "la maison qui fut autrefois à Philippe de Thurey" (1419 A.D.R. 10.G.84. fo 21v), "la maison du doyenné qui fut au défunt Seigneur Philippe de Thurey, archevêque" (1419. A.D.R.V10.G.84. fo28 et 33) etc. 


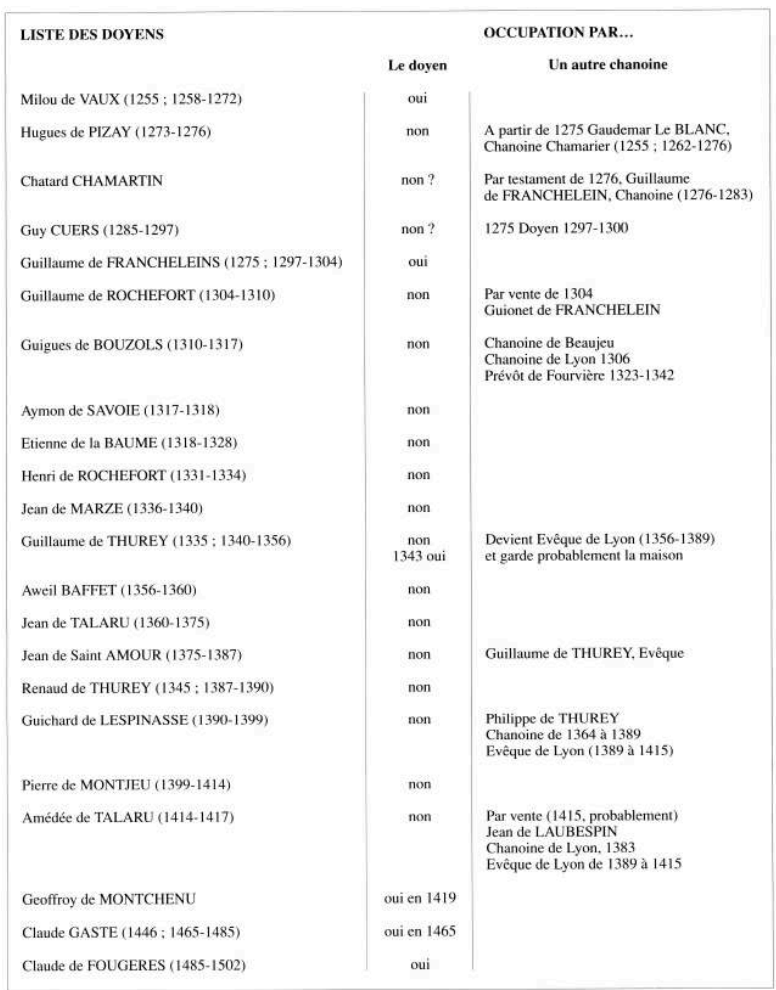

42 - Les occupants successifs de l'hôtel du Doyenné

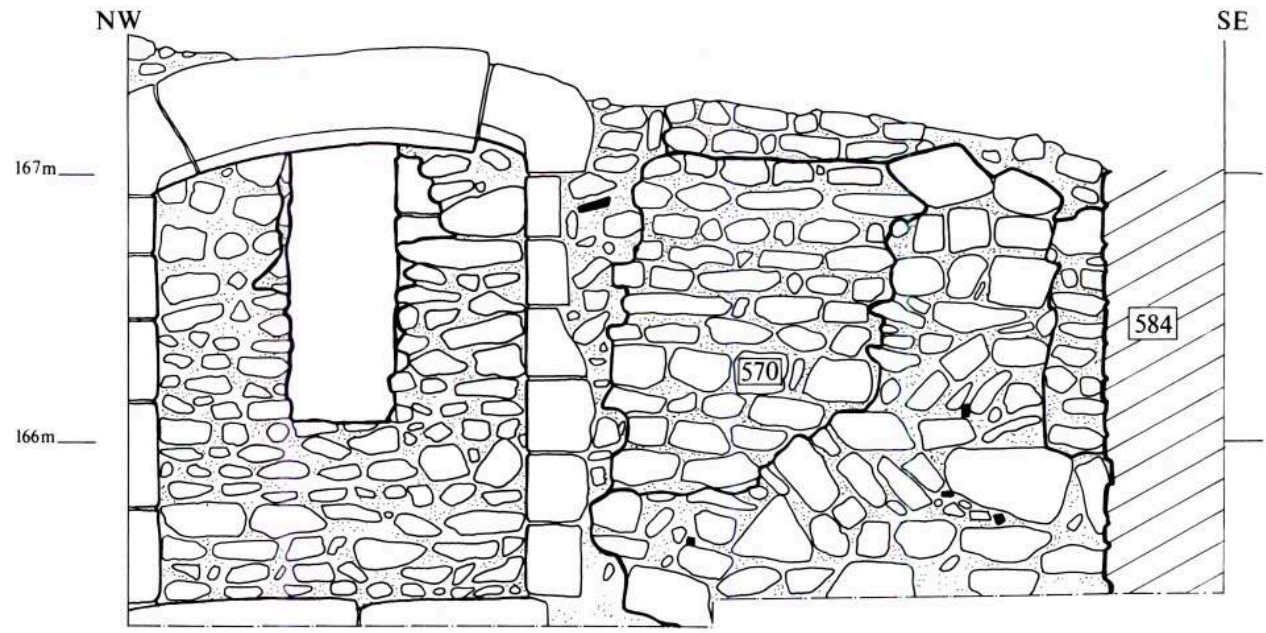

43 - Porte (US 577) avec un linteau en calcaire

\section{L'HABITAT INTRA-MUROS}

111 A l'est, dans le secteur ecclésiastique, un important bâtiment est construit au cours de cette période. Ses maçonneries massives (d'environ $1 \mathrm{~m}$ ) sont bien appareillées et liées au mortier. Les structures mises au jour se présentent de la façon suivante :

- une pièce A, dont les murs ouest (US 582-588), nord (US 570-554) et est (US 771), conservés en élévation, délimitent une pièce dont l'accès se fait par une porte (US 577) installée dans le mur nord, (voir fig. 40). Le linteau, un arc surbaissé et les jambages sont en calcaire coquillier gris ; ils sont chanfreinés sur la face sud, la pierre étant taillée plus sommairement sur la face nord, sans décor (fig. 43). Deux degrés, toujours en calcaire coquillier gris, font 
communiquer la pièce A et un espace situé au nord (B). A l'ouest de la porte, dans le mur nord (US 570), un bouchage présentant une limite de mortier très nette pourrait correspondre à une fenêtre condamnée ultérieurement.

- l'espace B, est limité à l'ouest par le prolongement (US 584) du mur ouest du secteur A (US 582-588). Ces murs présentent sur le parement ouest un enduit lissé de couleur brune. Une deuxième maçonnerie (US 585-586) vient doubler cette façade ouest et s'appuie sur l'enduit. Ces deux murs accolés parallèlement présentent un aspect massif: leur épaisseur est supérieure à $2 \mathrm{~m}$. Cette maçonnerie (US 585-588), qui vient doubler le mur de la maison canoniale, est un vestige du rempart (fig. 44). La maison canoniale, hôtel du Doyenné, serait donc antérieure à 1165-1180, date supposée de la construction du rempart. Pour la pièce A, la présence d'une fenêtre, l'aspect de la porte (ouverture de façade), ainsi que l'enduit de la façade ouest permettent d'interpréter cette pièce comme un rez-de-chaussée. Le secteur B était un espace extérieur, sans doute une cour.

Rue Carriès, a été mis au jour un mur orienté sud/nord qui présente un retour d'angle est/ouest dans la paroi sud de la tranchée. Ce mur est fondé sur le dallage de la voie gallo-romaine (période 3). Le parement intérieur est du mur, conservé sur cinq assises, est construit en appareil quadrangulaire lité, de facture assez grossière et lié au mortier. L'épaisseur du mur reste inconnue car l'emprise du chantier de génie civil ne s'étendait pas au-delà de cette maçonnerie. La confrontation de la localisation et du tracé de ce fragment de mur avec le parcellaire du quartier et les alignements de rue, permet de l'interpréter comme une partie du mur sud de l'enceinte et même de son angle sud-ouest, entre la rue Tramassac et la rue Carriès.

Les vestiges du mur d'enceinte retrouvés le long de l'ancienne rue Carriès et dans l'îlot Tramassac contre l'hôtel du Doyenné permettent, en prolongeant leur axe respectif, d'établir un angle dans le tracé du mur d'enceinte à l'est de la rue. Cette supposition est confirmée par plusieurs sources archivistiques. Un texte de 1466 (A.D.R.10.G.836) précise qu'une maison (3, rue Carriès) se trouve "dans la ruelle allant de la rue du Gourguillon à la porte du Cloître de l'Eglise de Lyon appelée de Tiers (rue Carriès) jouxtant les murs du Cloître de ladite Eglise au Nord, la ruelle étant entre les deux". Cette indication reprise plusieurs fois dans les textes postérieurs, établit que le mur d'enceinte constitue effectivement la façade de l'ancienne rue Carriès.

114 Cette source est complétée par l'examen des plans anciens (A.M.L. 3.S.313, 3.S.477, 1.S. 192) sur lesquels apparaît en effet une chicane dans le tracé ancien de la rue Carriès. D'une part, le corps de logis nord de la parcelle du Doyenné apparaît sur ces plans en saillie importante par rapport à l'alignement nord de cette rue. Cette façade principale du Doyenné se trouverait aujourd'hui au milieu de l'actuelle rue Carriès, à 5 m environ en avant de l'alignement nord des façades; le bâtiment de l'hôtel du Doyenné fermait donc la rue Carriès en impasse. 


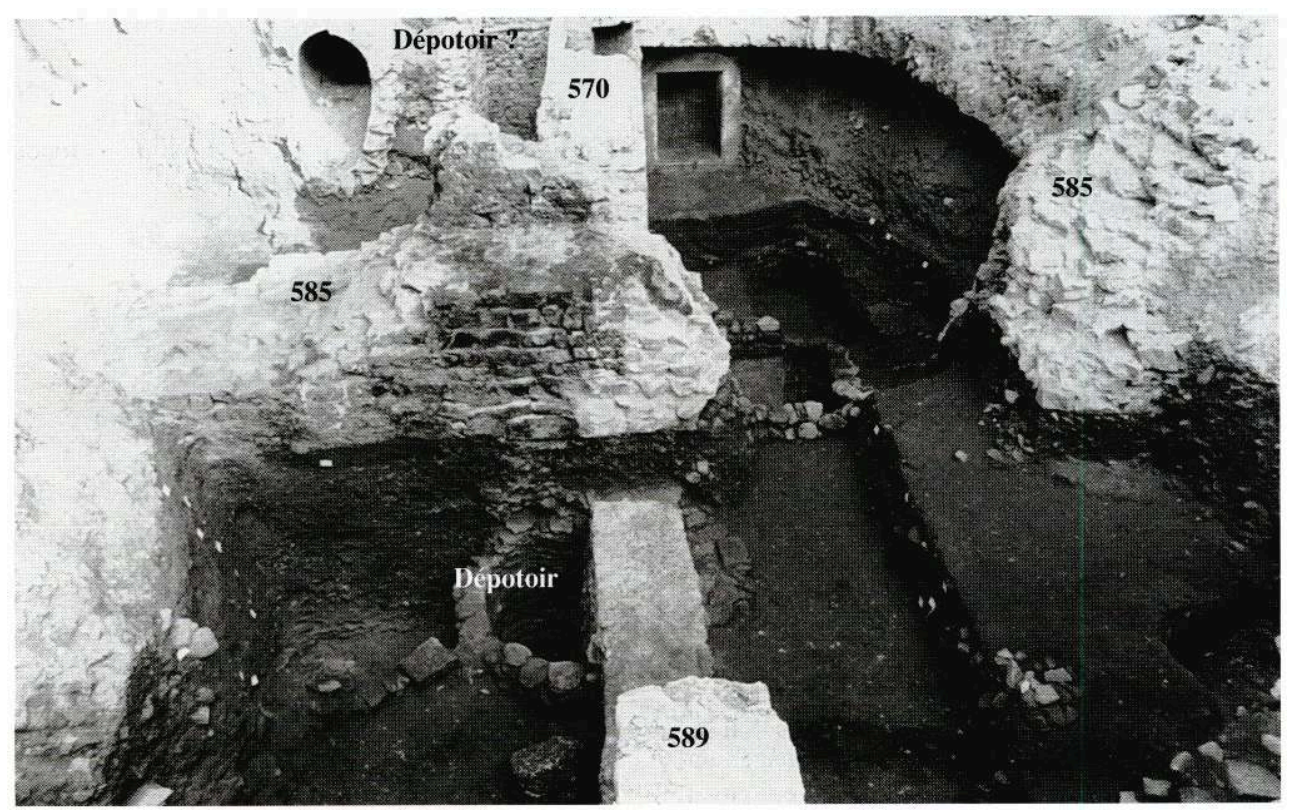

44 - Mur d'enceinte (US 585, 588) et puits

115 D'autre part, la présence d'une porte à cet endroit est mentionnée dans les textes dès 1395 (A.D.R. 10.G.840) : “Et d'abord la porte de Tyait, qui est contre la maison de l'Archevêque de Lyon (près de) la maison qu'habite Gilet d'Albon et qui mène au Gourguillon". La confrontation de ces documents avec le Plan scénographique permet de placer cette porte à l'est de la rue Carriès, contre l'hôtel du Doyenné et de supposer qu'elle s'ouvrait dans le tronçon est-ouest du mur d'enceinte, ce qui revient à modifier quelque peu le traçé proposé par Sachet (voir fig. 9) ; desservie par cette voie, elle ménage donc un passage sud/nord en chicane, débouchant à l'intérieur de l'enceinte sur une rue passant entre le Doyenné et la maison canoniale contiguë à l'hôtel d'Albon. Selon cette hypothèse, les dimensions évoqueraient plutôt une poterne, ce que confirme l'absence de figuration sur le plan d'une fortification pour cet accès.

\section{PÉRIODE 15}

\section{L'HABITAT EXTRA-MUROS}

\section{Les maisons de la rue Tramassac}

De nouveaux bâtiments sont aménagés. Les murs, conservés en élévation, sont liés au mortier et présentent un appareil relativement irrégulier avec des matériaux hétérogènes (moellons de calcaire, schistes, galets du Rhône, briques...).

La fouille a permis d'identifier deux parcelles accolées Tune à l'autre dont le découpage remonte vraisemblablement à la période précédente (fig. 45).

- La parcelle D, au sud, allongée, est occupée par deux corps de bâtiments, l'un sur rue, l'autre en fond de parcelle, séparés par une petite cour. La maison sur rue est reconstruite sur les fondations de l'habitat précédent; par contre la maison arrière est créée ex nihilo durant cette période.

- La parcelle E, au nord, comporte deux pièces contiguës, l'une à l'ouest, l'autre à l'est, l'ensemble formant le bâtiment sur rue. Le reste de cette parcelle se trouve hors de l'emprise de la fouille. 
$118 \mathrm{Au}$ cours de cette période, un important remblai, témoin d'un exhaussement spectaculaire des niveaux de la rue (US 254), provoque la transformation des rez-dechaussée en caves.

119 Le large mur de la période 14 (US 892-895) est démonté par endroits jusqu'au niveau de la voie romaine sur laquelle ses fondations s'appuyaient afin d'en récupérer les pierres, comme l'atteste une large fosse.

Les caves ne sont pas encore voûtées, mais seulement séparées des rez-de-chaussée par des planchers. Ce phénomène est attesté pour d'autres maisons de la rive droite de la Saône bien qu'il soit difficile de le constater, car l'épaisseur des voûtes installées ultérieurement couvre souvent les trous d'ancrage des poutres. L'accès aux caves s'effectue alors soit par une trappe et une échelle, soit par la cour intérieure de la parcelle E. Une ouverture (US 863), bouchée ultérieurement, relie entre elles les caves des bâtiments des deux parcelles.

121 Suite à l'exhaussement du niveau de sol, chaque maison possède un sous-sol ; en fait, l'ensemble même de la parcelle est transformé en niveau de cave, sous la cour comme sous le bâti. Les niveaux de sol de ces espaces aménagés en sous-sol ne sont pas conservés; ils ont été recreusés au XVIe s. lors de nouveaux aménagements. Il faut également ajouter que la fouille du niveau de rue (US 254) aménagé sur les couches de remblais n'a pas permis de mettre au jour un matériel qui permette de préciser les datations. En effet, l'ensemble des monnaies retrouvées dans les couches entre les niveaux de rue à cette période sont d'époque gallo-romaine; ce matériel résiduel montre que ces couches sont un apport volontaire et non une accumulation de sédiments.

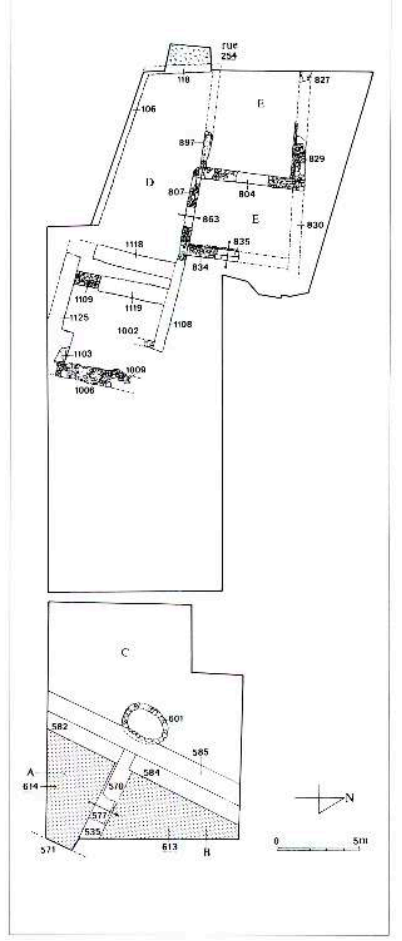

45 - Plan des vestiges de la période 15 
Dans les démolitions du XIXe s., plusieurs pierres taillées provenant des carrières des Monts d'Or ont été retrouvées; ces éléments appartenaient à des jambages de portes et de fenêtres et certains d'entre eux étaient décorés de moulures prismatiques, utilisée du XIVe et XVIe s.

Si l'on regroupe les données des périodes 14 et 15 , on constate que l'habitat se développe dans un premier temps à l'intérieur d'un parcellaire peu divisé (fig. 46). Les transformations sont principalement dues aux différents jeux des acquisitions, partages, héritages, ventes. Ces changements laissent des traces visibles dans l'évolution des structures.

L'évolution du tènement situé dans la partie ouest de la zone de fouille, ayant façade sur la rue Tramassac, est très révélatrice de l'évolution du parcellaire sur la rive droite de la Saône. En effet, jusqu'au milieu du XIVe s., ce tènement est rattaché à un autre situé plus au sud et doit s'étendre jusqu'au mur de clôture au nord (période 14). En 1303, date du premier texte d'archives exploitable, un seul propriétaire, Simon d'Albon, est mentionné pour l'ensemble. Ce n'est qu'en 1371 que ce tènement, appartenant toujours à la famille d'Albon, est divisé en trois parcelles; deux d'entre elles sont situées dans l'emprise des fouilles à l'ouest (parcelles D et E).

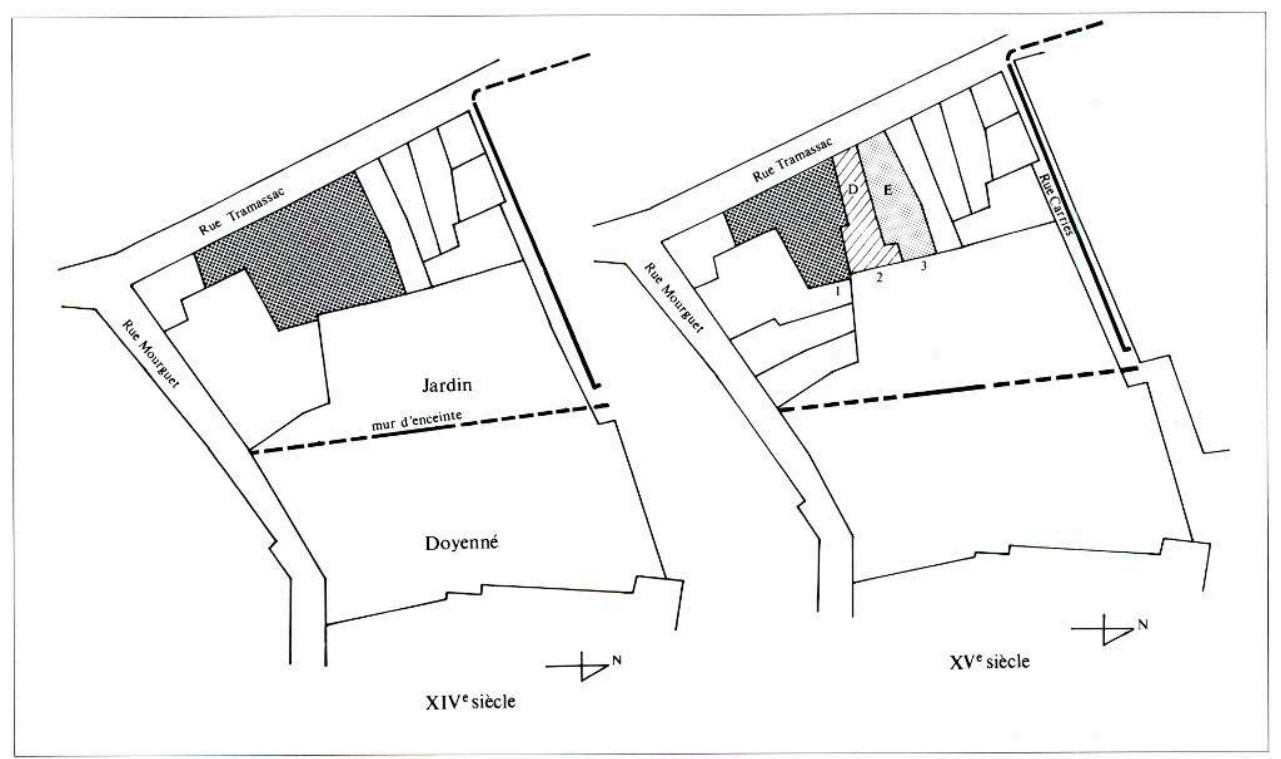

46 - Schéma d'évolution du parcellaire D et E

Le partage est réalisé dans le sens est-ouest afin que chaque parcelle ait un accès sur la rue Tramassac.

A l'intérieur des parcelles, le bâti se densifie et adopte le plan type, soit deux corps de bâtiments séparés par une cour. A cette époque les descriptions faites dans les textes sont très succinctes ; il est fait mention de maisons "haute et basse" ou "haute, moyenne et basse", c'est-à-dire comprenant deux à trois niveaux d'élévation. En 1475, la parcelle nord est décrite ainsi : "domum altam bassam cum curte et putheo" (A.D.R $10 \mathrm{G}$ 988.46). En 1493, la même maison est dite "haute, moyenne et basse". Un niveau supplémentaire a donc été créé entre 1475 et 1493 . Quant à la parcelle sud, elle est dite "domum altam bassam" (A.D.R. $10 \mathrm{G}$ 988.14) et comme la parcelle nord, "aulte, moyenne et basse" en 1493 (A.M.L. CC.5.27 vo). 


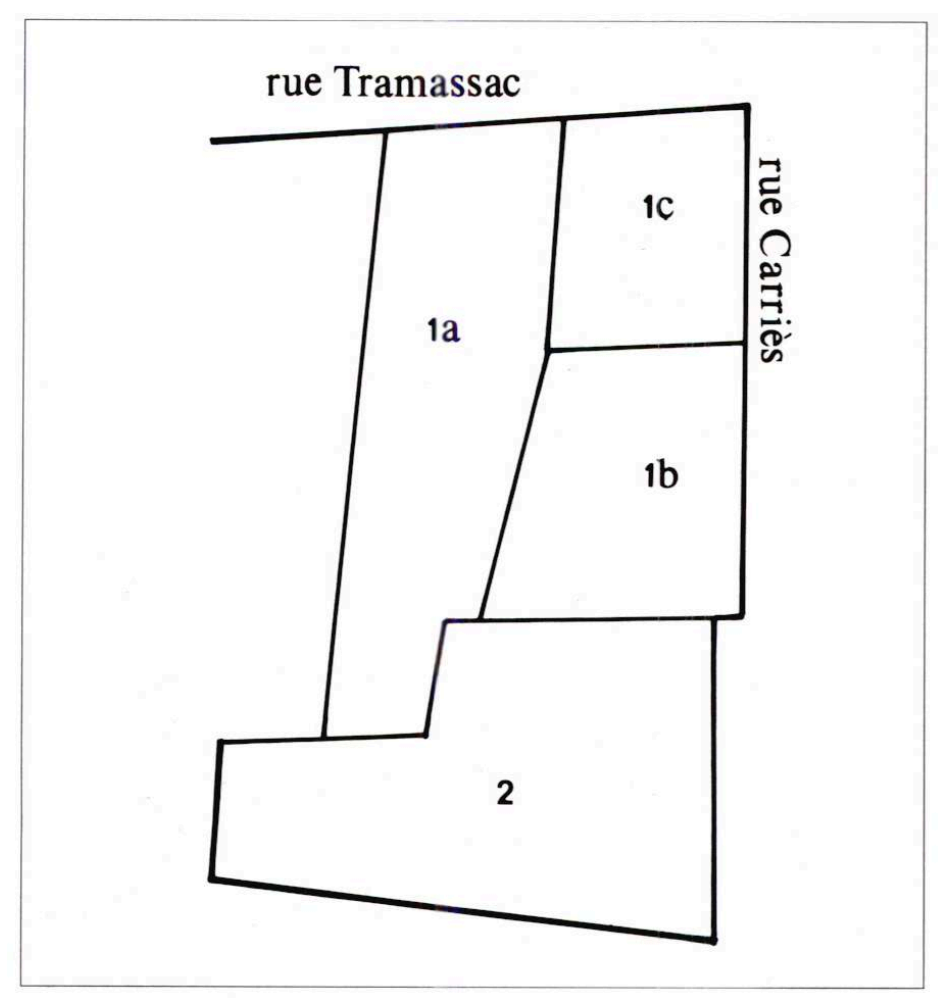

47- Schéma des parcelles formant l'angle des rues, Carriès et Tramassac

\section{Le tènement du 5 rue Carriès}

Le tènement situé 5 rue Carriès, à l'angle des rues Tramassac et Carriès, apparait à la fin du XVe s. dans les textes d'archives. Ce sont les premières mentions écrites connues, ce qui ne signifie pas que les bâtiments apparaissent à cette époque. Après confrontation d'un terrier du XVe s. (terrier Guibert 1464-1500. A.D.R. 10.G.988) et d'un parangon de terriers anciens, en grande partie disparus (A.D.R. 10.G.1000), l'évolution de ce tènement peut être restituée (fig. 47).

En 1466 (terrier Guibert), Etienne Durtières, potier d'étain, reconnaît trois parcelles jointives, soit un seul tènement sur lequel sont bâties plusieurs maisons : une maison haute et basse (1a), une autre maison haute et basse (1b), et enfin une troisième maison haute et basse qui fait "l'angle de la rue du Gourguillon et de la ruelle allant de cette rue à la porte du cloitre" (1c) (fig. 48).

129 En 1472, Jean de Roche, clerc de notaire, reconnait la parcelle contiguë (2), soit une maison "haute et basse", confinée à l'ouest par la maison d'Etienne Durtières et à l'est par les jardins du doyen. 


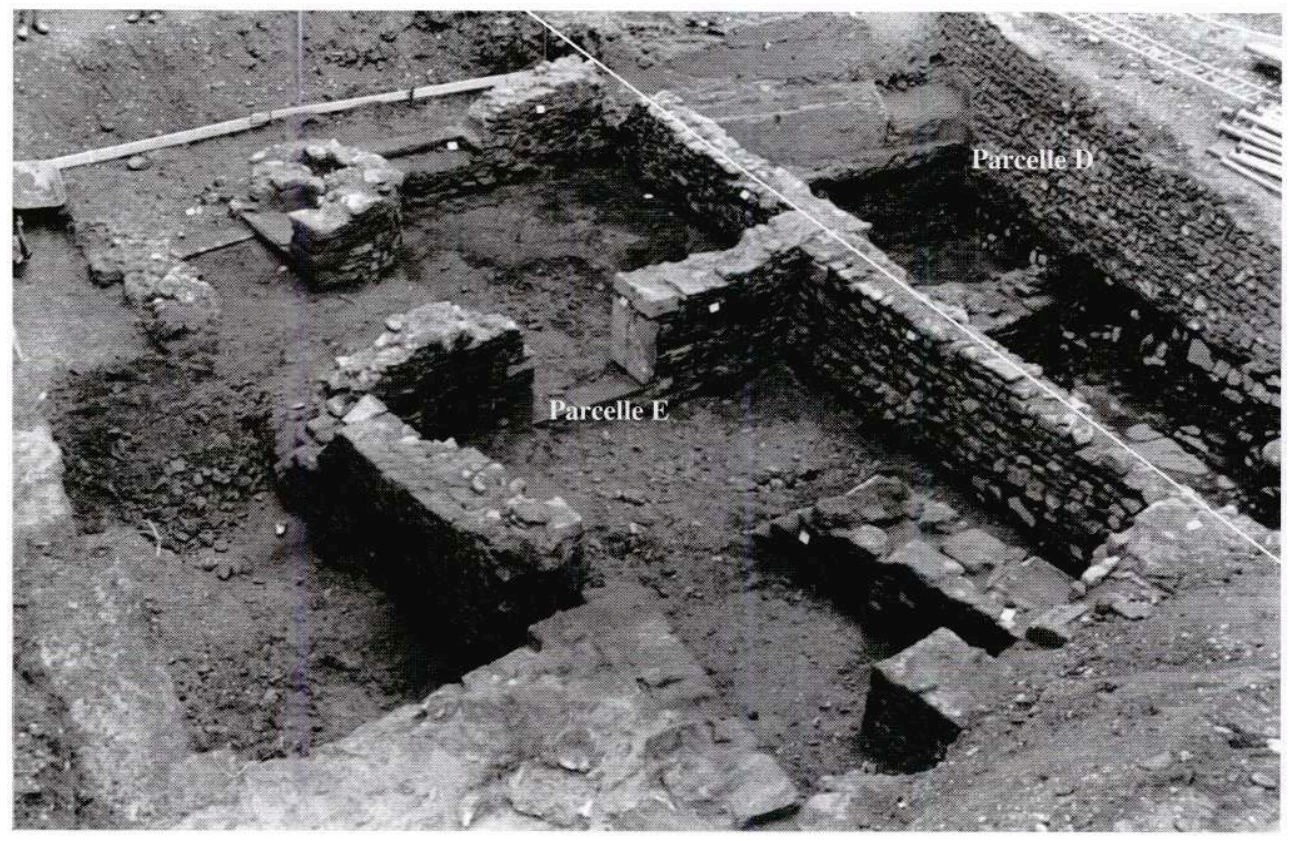

48 - Vue d'ensemble des structures, rue Tramassac, depuis l'ouest

\section{L'HABITAT INTRA-MUROS}

Dans la zone est de l'îlot Tramassac, les bâtiments intra-muros ne semblent pas subir de transformations quant à la distribution des espaces. Le phénomène de rehaussement des niveaux, observé rue Tramassac, n'est pas encore attesté à l'intérieur de l'enceinte.

Le puits accolé au mur d'enceinte ne fonctionne plus en tant que tel dès le XVe s. L'imposante quantité de matériel retrouvée dans les couches de comblement montre qu'il est alors utilisé comme fosse d'aisance et dépotoir. En effet, la présence de pots intacts ou quasiment entiers sans ébréchures prouve qu'ils ont dû rencontrer une couche de matière organique qui atténuait le choc de la chute. De plus, les nombreuses couches de chaux dans le comblement attestent la nécessité d'assainir cet endroit.

Pour cette période, allant de la fin du XIIIe au XVe s., les archives nous renseignent sur le bâtiment du Doyenné, même s'il n'existe aucune visite détaillée dans les textes de cette époque. Entre 1275 et 1419, cette maison semble avoir la prédilection des doyens, bien que le bâtiment ne soit pas officiellement dévolu à leur dignité (voir fig. 42). Il existe toutefois plusieurs exceptions, particulièrement entre 1356 et 1419, et ce n'est qu'en 1426 que l'hôtel est définitivement rattaché à la fonction de doyen. La maison est cependant en très mauvais état au milieu du XVe s., "en ruine en plusieurs endroits murs et toits" (10 G 96 fo253. 23 XII 1468). Elle a nécessité de la part du doyen G. de Montchenu, puis de son successeur, des réparations très onéreuses.

133 A l'ouest de la muraille, au centre de l'îlot, un jardin est mentionné en 1419. Le puits transformé en dépotoir comporte plusieurs couches de comblement (US 711,710, 685, 679) qui présentent un intérêt considérable dû à la grande quantité et variété de matériel céramique et verre, retrouvé parfois intact (fig. 49). Dans les couches inférieures, le mobilier présente des éléments de vaisselle de table datés de la fin du XVe s. Quant aux couches supérieures du remplissage (US 685-679), elles ont pu être datées du XVIe s. par la présence de deux salières en faïence et de marmites à pâte grise et à anse coudée. 


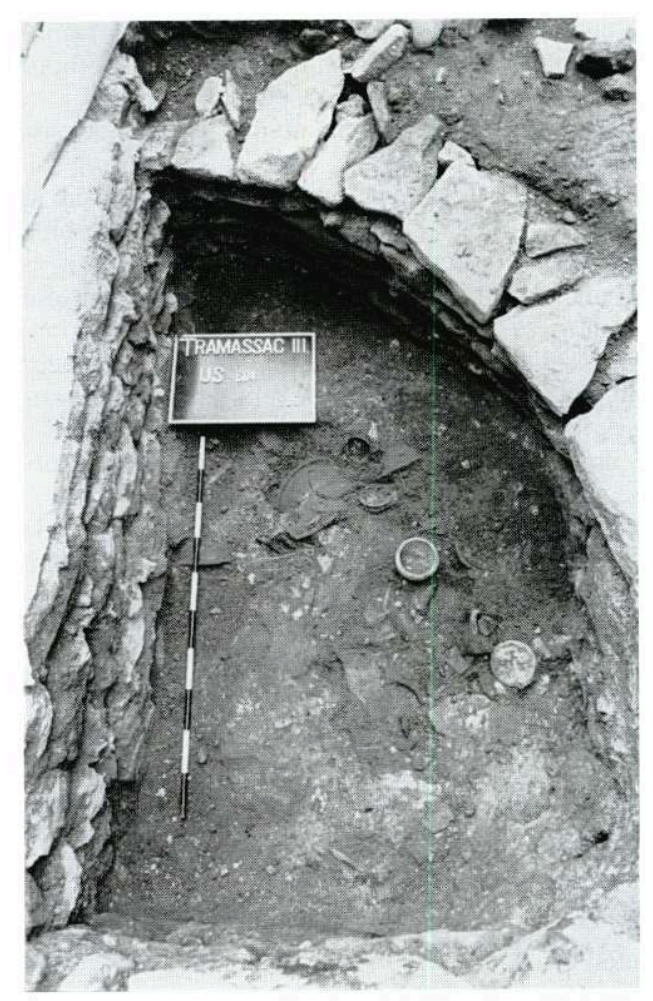

49 - Puits transformé en dépotoir

Parmi les fragments de verre, plusieurs formes ouvertes ont été identifiées (fig. 50) :

- des verres à pied sans décor (type $\mathrm{C}$ ), moulés en une seule paraison et dont le pied est formé par resserrement de celle-ci ;

- une coupe à pied tronconique (type $\mathrm{C5}$ ), façonnée également en une seule fois, et dont le bord est ourlé et décoré d'un fdet bleu ;

- des gobelets, moulés en une fois, décorés de pastilles en relief (type B2) ;

- un verre à tige pleine ornée d'un bouton bleu, la coupe droite est couverte de frises de verre bleu rapportées (type D) ;

- la typologie des formes fermées, ont été identifiées :

- un col sans décor de bouteille à vin ou à liqueur (type G2) de forme allongée reposant sur une panse globulaire à fond concave ;

- un col (type G2), orné d'un filet de verre rapporté après soufflage ;

- un col évasé et un fond concave qui ont permis de déterminer un urinal (type G4). (A la fortune du pot 1990-1991 ; A Travers le verre 1989 ; Auger 1990). 


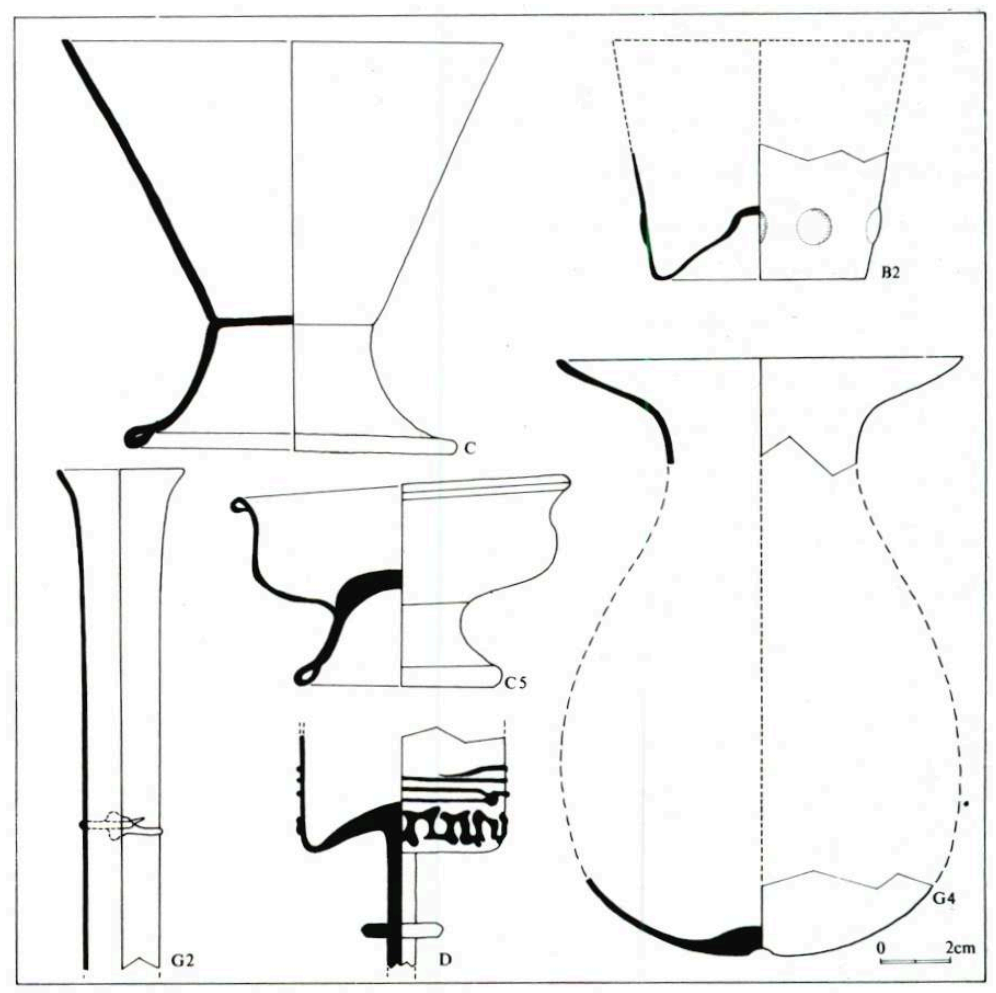

50 - VERRES

Dans ce même dépotoir, il faut également ajouter la découverte d'une grande quantité d'ossements d'animaux, parmi lesquels des éléments de squelettes de deux jeunes chiens, dont l'étude ostéologique a révélé des traces d'équarissage sur certaines parties, qui ont pu être opérées à des fins alimentaires; bien que cette observation soit occasionnelle sur le site, la consommation de viande de jeunes chiens est bien attestée dans le monde médiéval. Parmi les autres ossements, les restes des trois principales espèces domestiques sont représentés en proportions presque équilibrées : d'après le poids de ces restes, le bœuf est l'espèce prédominante à plus de $60 \%$, suivi par ordre d'importance par le porc et les ovicapridés. L'analyse ostéologique a permis d'observer que dans ses lignes générales, la gestion des animaux domestiques semble fonctionner selon les règles perpétuées de siècle en siècle. Elle semble néanmoins évoluer dans le cas de chaque espèce vers un élevage de plus en plus spécialisé. La conservation de bovins adultes, voire séniles, s'accorde avec leur utilisation au travail, celle des ovicapridés adultes avec la production de lait et de laine, alors que le porc est exclusivement destiné à la boucherie. Il s'agit d'un élevage adapté aux divers besoins, qui tire le meilleur profit des produits animaux. 


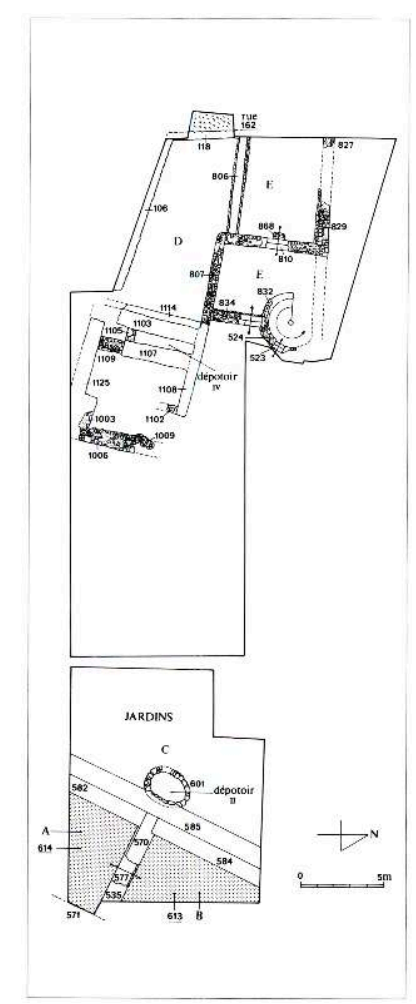

51 - Plan des vestiges de la période 16

\section{PÉRIODE 16}

Cette période comporte des modifications à l'intérieur des parcelles de l'habitat laïc, rue Tramassac (fig. 51). Certaines circulations sont modifiées. Notamment l'ouverture reliant les caves des deux parcelles est condamnée par un bouchage en maçonnerie, et une nouvelle ouverture entre les deux caves de la maison sur rue de la parcelle E est aménagée ; les montants et le seuil (US 810) de cette porte sont en calcaire coquillier gris et l'arc de décharge est en briques liées avec un mortier de chaux. De plus un escalier en vis (US 524) en calcaire des Monts d'Or est construit; il relie les différents niveaux des bâtiments de la parcelle E (fig. 52). Il repose sur un important blocage de maçonnerie qui sert à stabiliser l'ensemble, compte tenu de la masse et de la structure de l'escalier. Ce blocage perce toutes les couches antérieures jusqu'à l'arase supérieure du mur 892-895 (période 14). 


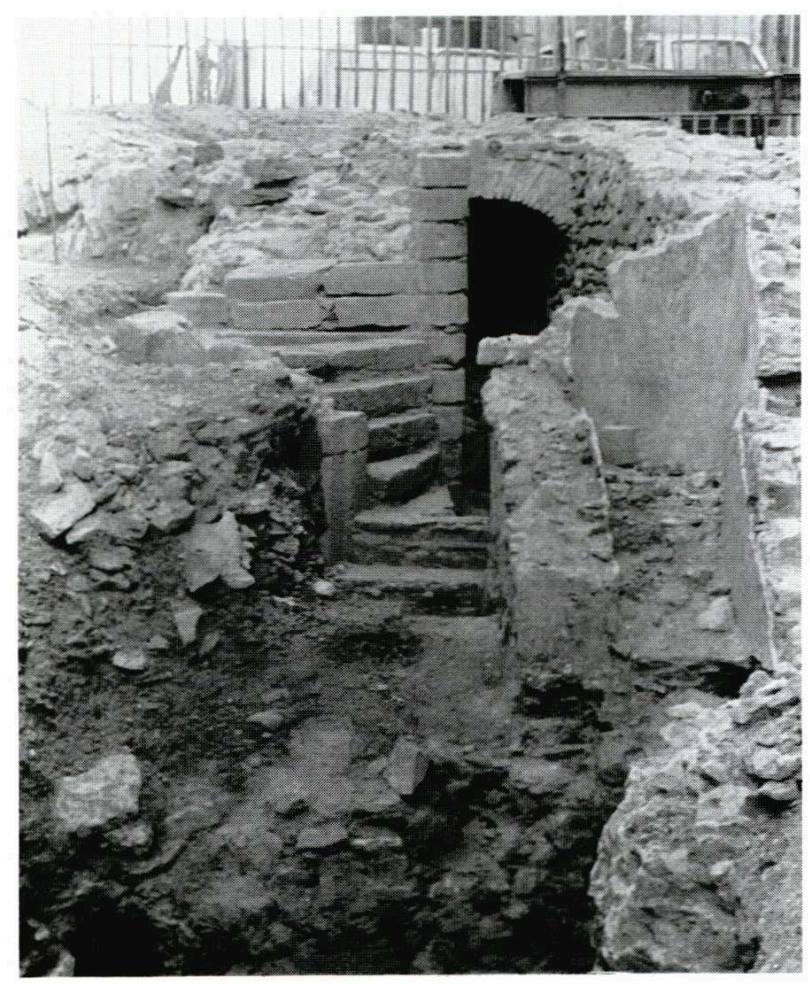

52 - Escalier en vis de la parcelle E, rue Tramassac

137 L'espace situé entre les deux bâtiments de la parcelle D est utilisé comme dépotoir à l'exclusion de sa partie sud, qui forme un passage entre les deux corps de logis. Le dépotoir, utilisé jusqu'au début du XVIIe s., a livré du matériel en très bon état, parmi lequel du verre dont l'éventail des formes va de la fin du XVe s. au milieu du XVIe s. On a retrouvé (fig. 53) :

- un gobelet (type B) moulé en une seule paraison, un autre portant un décor de cordon pincé, rapporté à la base dont la panse est ornée de côtes moulées en relief ;

- de nombreux verres à pied (type $\mathrm{C}$ ) sans décor, un verre à pied (Type $\mathrm{Cl}$ ) dont la panse porte un motif d'alvéoles moulé en relief, un verre (type C2) orné de plusieurs filets blancs rapportés, sur le haut de la panse ;

- un bol hémisphérique (type G5), un urinal (type G4) et une aiguière (type G3) identifiée grâce à son bec verseur, son col et sa anse. 


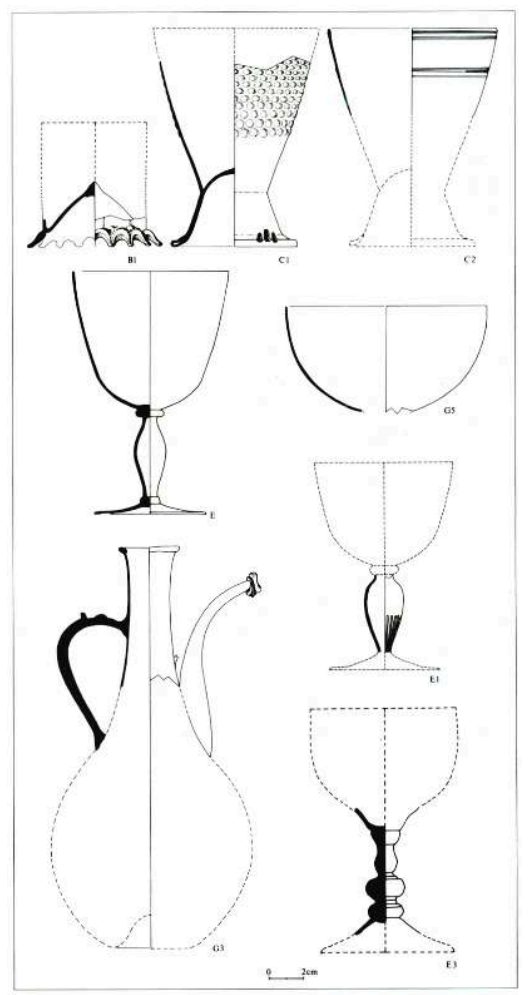

53 - Verres

Au milieu du XVIe s. apparaissent des verres à jambe creuse ovoïde (type E) parfois ornés de côtes parallèles en relief (type E1), des jambes moulées d'un décor de tête de lion (type E2) et des verres à jambe pleine, dite "balustre", décorée de plusieurs boutons (type E3). La coupe de ces verres peut avoir la forme d'un calice, les parois sont parfois droites ou obliques.

139 Le lot de céramiques, daté fin XVIe - début XVIIe s., contient de nombreux éléments de vaisselle culinaire à pâte grise et à anse coudée, ainsi que des éléments du type "service vert" qui sont très répandus dans la région à partir du XVIe $s$.

140 Une nouvelle phase de remblaiement surélève le niveau de la rue (US 162) et les caves des maisons situées en bordure sont alors totalement enterrées.

141 L'hôtel du Doyenné ne subit pas de transformations majeures et l'ancien puits continue à être utilisé comme dépotoir.

\section{PÉRIODE 17}

\section{L'HABITAT EXTRA-MUROS}

\section{Les maisons de la rue Tramassac}

Le parcellaire de la zone fouillée extra-muros, rue Tramassac, reste tel qu'il est fixé depuis le XIVe s. Les archives apportent peu de renseignements. Un texte, du 7 novembre 1640, décrit la parcelle nord (E) en ces termes: "une maison contenant deux corps, une cour au milieu" (A.D.R $10 \mathrm{G} 978$ 17); un autre texte, du 30 décembre 1684, qualifie l'ensemble "d'extrêmement vieux" (A.D.R. $10 \mathrm{G} 974$ 76). Quant à la parcelle sud (D), la description du 6 décembre 1681 est très succincte : "maison haute moyenne et basse en plusieurs membres" (A.D.R. $10 \mathrm{G}$ 993.8). Les modifications apportées durant le XVIe et 
le début du XVIIe s. concernent principalement les élévations des bâtiments. Durant cette période, l'ensemble des caves est voûté; ces voûtes ont été installées en sousœuvre avec un coffrage dont l'empreinte des planches est encore visible sur l'intrados des voûtes. Les pierres, en majorité des dalles de schiste, sont posées sur chant et liées au mortier (fig. 54).

La construction de ces voûtes atteste une volonté de consolidation. Ce renforcement peut s'expliquer par un surcroît de charge à la base des murs provoqué par la surélévation des maisons, phénomène courant dès la fin du XVe s. et le début du XVIe $s$. L'épaisseur des voûtes $(0,80 \mathrm{~m})$ et leur ancrage en pendentif sur les murs latéraux provoquent un abaissement du plafond des caves, qui empêche de circuler debout; aussi le sol est-il recreusé pour donner une hauteur convenable. Cette opération explique la disparition des niveaux d'occupation des caves des XIIIe - XVe s. et le fait que les fondations des murs soient très peu enfouies. Sur l'extrados des voûtes des caves, des remblais sont rapportés afin de niveler le sol et de constituer le plan de circulation du rez-de-chaussée. Plusieurs niveaux d'occupation successifs ont ainsi été mis au jour, ainsi que le soubassement d'un poêle (fig. 55).

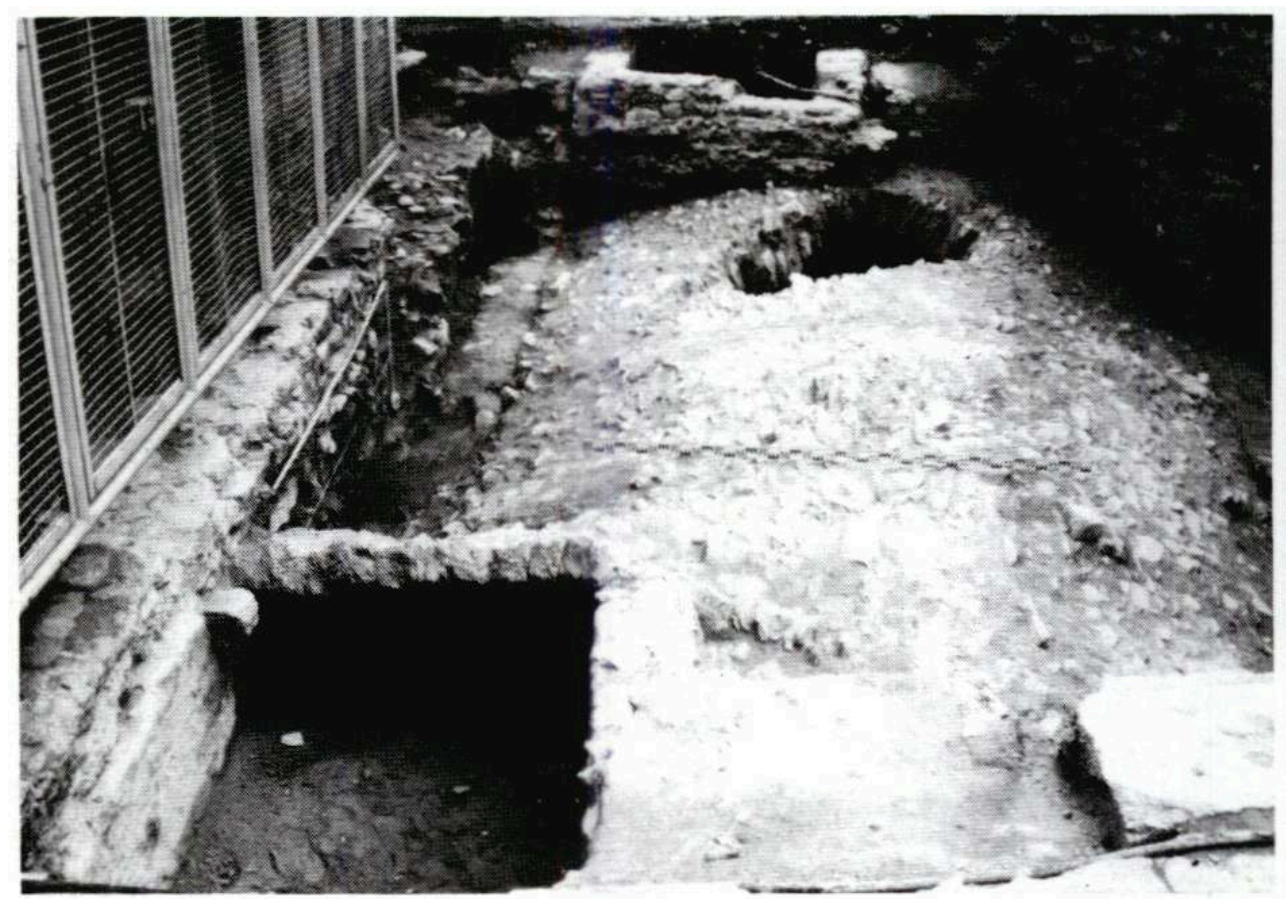

54 - Voûte de la cave de la parcelle D, rue Tramassac 


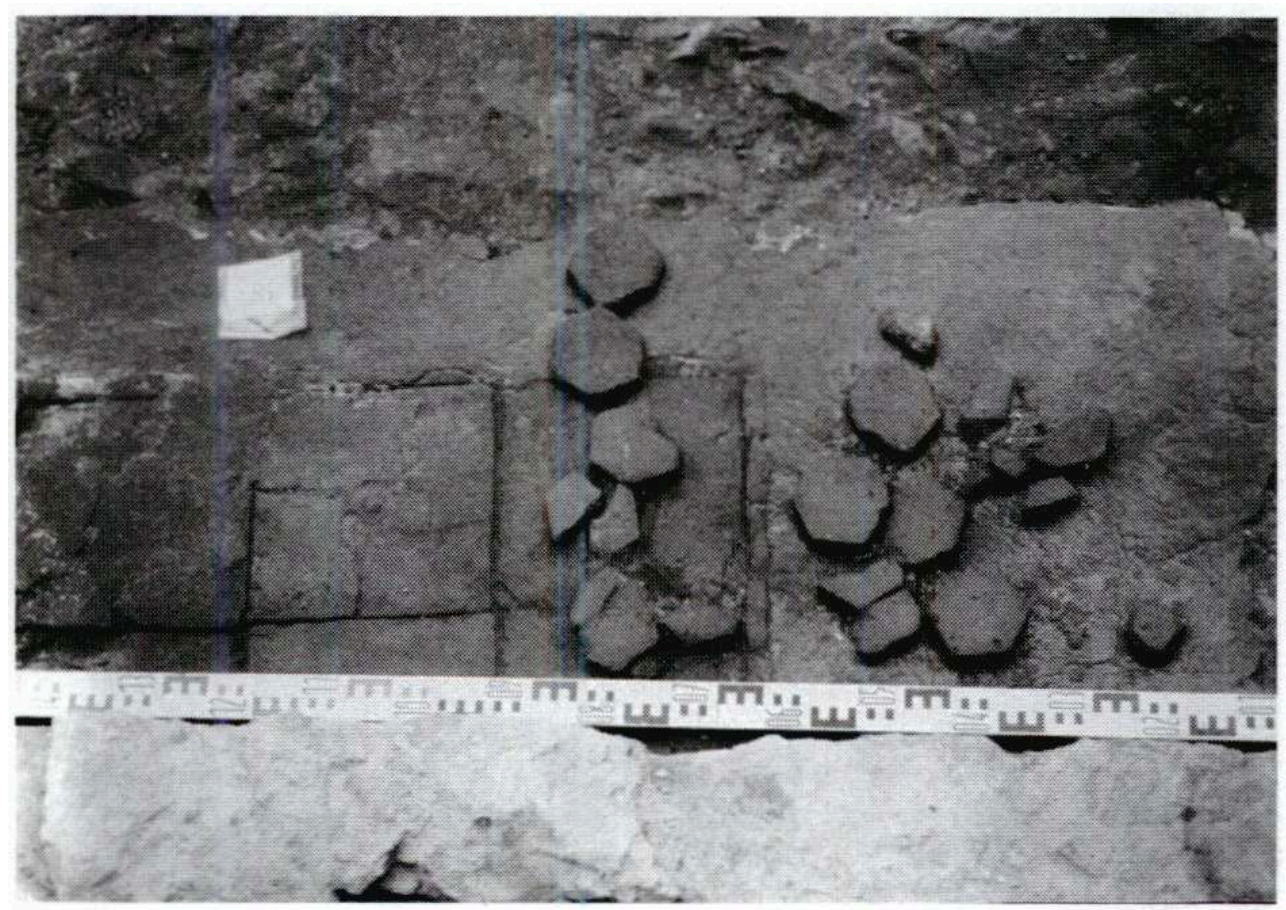

55 - Soubassement d'un poële, parcelle D, rue Tramassac

144 La parcelle E subit d'autres modifications; le mur nord de la maison sur rue est reconstruit et son axe est légèrement dévié (fig. 56). Il en résulte un léger rétrécissement de la parcelle qui oblige à remanier l'escalier. La forme arrondie est brisée et des marches de réemploi (US 511) sont posées sur les anciennes avec des reprises en blocage maçonné de manière à modifier la révolution de l'escalier. A ce stade, l'escalier n'est plus utilisable à tous les niveaux de la maison, mais seulement entre le rez-de-chaussée et la cave car l'espace laissé par ces transformations empêche une révolution normale de l'escalier. Un autre escalier a donc dû être construit pour accéder aux étages supérieurs depuis le rez-de-chaussée. 


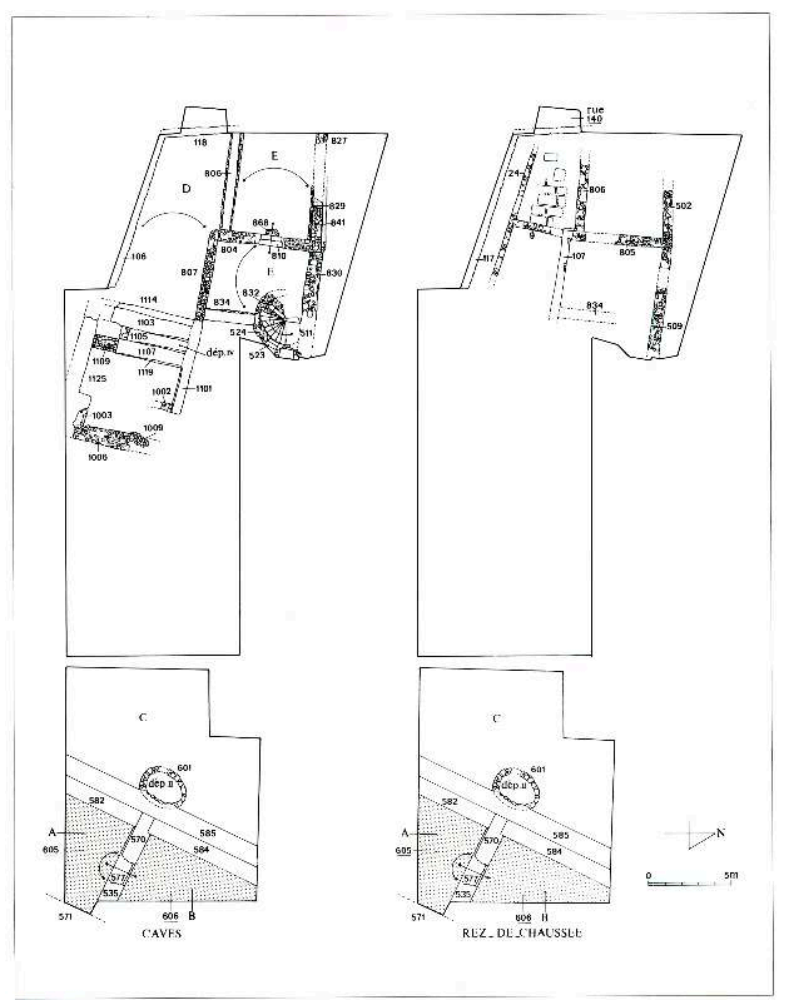

56 - Plan des vestiges de la période 17, caves et rez de chaussée

Une ouverture surmontée d'un arc de décharge en briques est aménagée afin de permettre le passage depuis l'escalier vers les caves de la maison sur rue. Un placard a également été découvert dans l'épaisseur d'un mur, accolé au noyau de l'escalier. Ce placard est construit en dalles de calcaire à entroques, surmonté d'un arc de décharge de même nature. Une nouvelle porte (US 523) est percée dans la cage d'escalier et permet d'accéder aux caves par la cour intérieure. L'ouverture qui remplissait cette fonction est abandonnée et bouchée.

Le matériel céramique retrouvé dans les couches d'occupation sur les extrados des voûtes permet de placer leur construction au début du XVIe s. A ce matériel, il faut aussi ajouter quatre monnaies de François 1er qui indiquent que ces constructions n'ont pu être réalisées avant son avènement en 1515. La présence d'un soubassement de poêle au rez-de-chaussée de la maison sur rue de la parcelle D est également un élément qui confirme ces datations.

\section{Le tènement du 5 rue Carriès}

Le tènement se transforme et se subdivise ; les parcelles sont reconnues comme suit : la partie (la) du tènement est désolidarisée de l'ensemble et forme une nouvelle parcelle. Dès 1516, date à laquelle elle est vendue par Etienne Durtières, une partie de cette maison appartient à Antoine Laguille et sa femme A. Descorey et l'autre partie à P. Aimonet; or ce dernier possède aussi les parcelles (1b) et (1c) (terrier Valentin). Ce n'est qu'à partir de 1547 que cette parcelle est définitivement indépendante de l'autre (terrier Tourveyon). Cette division est mentionnée dans un texte de 1619 (terrier Girinet) "une chambre, une arrière chambre, au premier étage avec les greniers dessus, lesdits 
biens sur le derrière et galerie qui fut des appartenances d'une maison située en rue de Gourguillon";

- les parcelles (1b) et (1c) forment un seul ensemble avec une maison dite "haute, moyenneet basse" en 1545. La parcelle (2), où une maison "haute et basse" est attestée en 1472, est décrite en ces termes en 1548: "une maison haute, moyenne et basse, jouxtant la rue de Tiers (rue Carriès) tendant du Gourguillon à la porte de Tiers"

L'ensemble a été entièrement remanié en façade en 1783 au moment où l'élargissement de la rue Carriès atteste une volonté d'aménager un système de voirie à l'intérieur d'un monde clos et de mettre en communication la rue Tramassac avec la Saône (A.D.R. 10 G 3814 ) (fig. 57, 58).

\section{L'HABITAT INTRA-MUROS}

Dans la zone intra-muros, le processus d'exhaussement des terrains déjà effectué dans la zone extra-muros se réalise progressivement.

Le Plan scénographique (voir fig. 7) donne une image de l'hôtel du Doyenné et de l'enceinte à cette période. Cet Hôtel est formé de trois corps de bâtiment: le corps principal semble être celui qui ferme la rue Carriès en impasse et dont la façade principale donne sur la place Saint-Jean. Il est relié à Test à un autre bâtiment possédant une galerie de façade; un troisième corps de logis (celui repéré en fouille) encore plus au sud en vis à vis du premier. A l'ouest, encadré par ces trois bâtiments, un espace non construit est clos à son extrémité ouest par le mur crénelé de l'enceinte capitulaire qui apparaît donc nettement à l'intérieur de l'îlot. Enfin, à l'ouest, de l'autre côté du mur crénelé, un espace vide est représenté qui correspond aux "jardins du Doyenné". Ces derniers sont séparés, des maisons de la rue Tramassac, à l'ouest, par les murs des bâtiments en fond de parcelles. Les jardins du Doyenné sont mentionnés comme confins orientaux dans chaque texte pour ces deux parcelles : en 1632, "jardin du Doyenné passage entre deux de matin" (A.D.R. $10 \mathrm{G}$ 978.17) et en 1682, "le jardin du Doyenné court entre deux de matin" (A.D.R. $10 \mathrm{G}$ 872.13).

151 Un autre document, "l'anonyme" Fabriczy (fig. 59), confirme cette lecture du Plan scénographique : le dessin montre le quartier canonial vu de l'ouest, depuis les pentes de la colline ; au premier plan est représenté l'îlot Tramassac. L'enceinte crénelée apparaît nettement au milieu de cet îlot, avec des espaces vides à l'ouest (jardin) et à Test (cour). Tous ces éléments confirment l'interprétation des données de terrain sur l'îlot depuis les XIIe et XIIIe s. (période 14). 


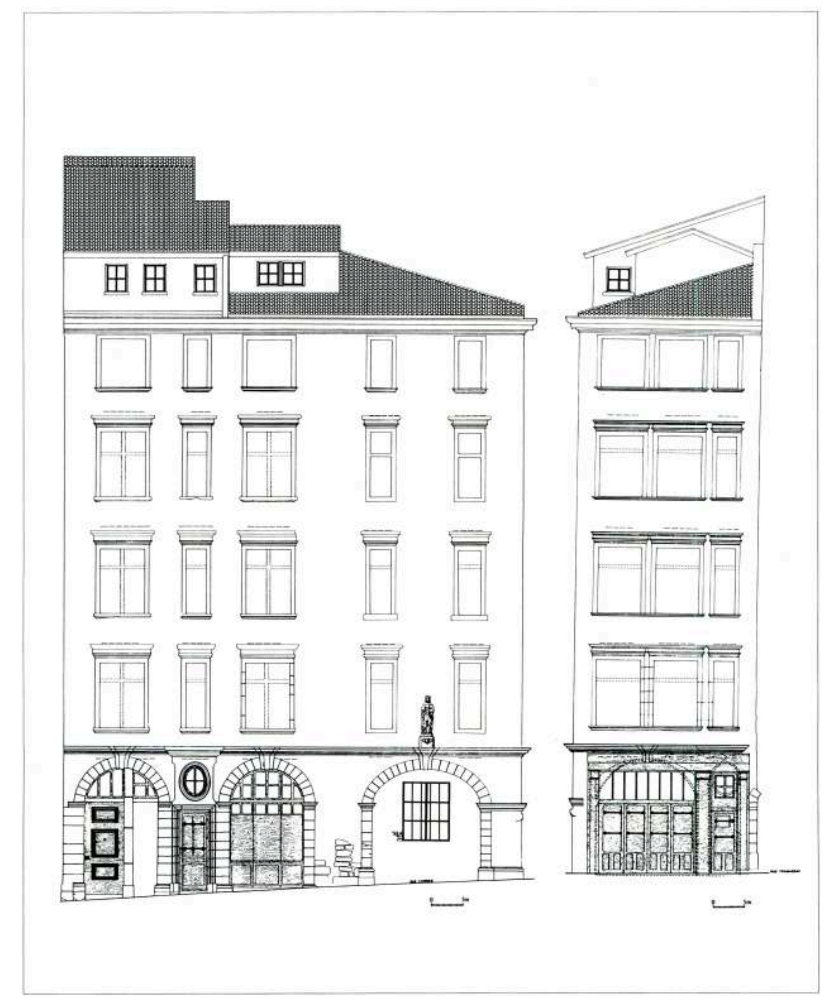

57, 58 - Relevé de la façade de l'immeuble situé à l'angle de la rue J. Carriès et de la rue Tramassac Cet immeuble reste le seul témoin ancien encore en élévation dans cette rue, les autres ayant disparu à la suite d'un alignement réalisé au XIXe s.

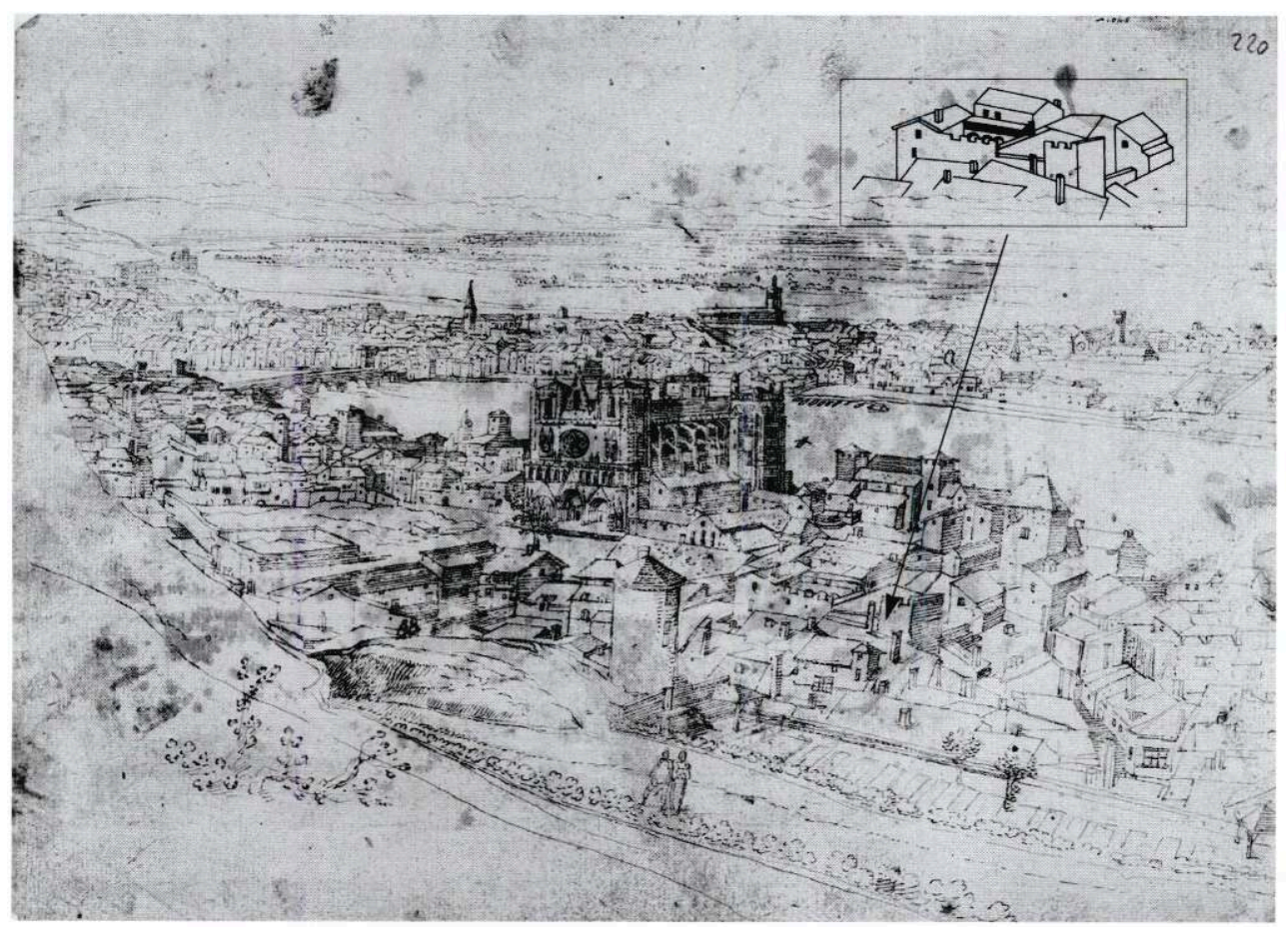

59 - Le quartier canonial de Lyon vu du sud-ouest. Anonyme Fabriczy "Lyon" Braune Feder - 20,5 × 28,5 cm. STAATSGALERIE STUTTGART - Inv. Nr 5789, recto 


\section{PÉRIODE 18}

\section{L'HABITAT EXTRA-MUROS}

\section{Les maisons de la rue Tramassac}

Dans les deux parcelles $\mathrm{D}$ et $\mathrm{E}$, des escaliers sont aménagés en façade, qui relient directement la cave à la rue. Des ouvertures sont percées dans les voûtes pour installer ces escaliers droits (fig. 60 à 61).

Dans la parcelle D, l'escalier est construit contre le mur mitoyen où un corbeau est installé pour soutenir l'arc de décharge sous la voûte.

Dans chaque parcelle, une pile est construite contre le mur de façade pour consolider les voûtes affaiblies. Ces transformations pourraient être liées, au moins pour la parcelle $\mathrm{E}$, à un alignement de façade. Des mentions d'autorisation d'aligner, répétées à plusieurs reprises en 1682 et en 1685, en témoignent (A.M.L. DD 36-156 ; DD 47-135 ; DD 47-178).

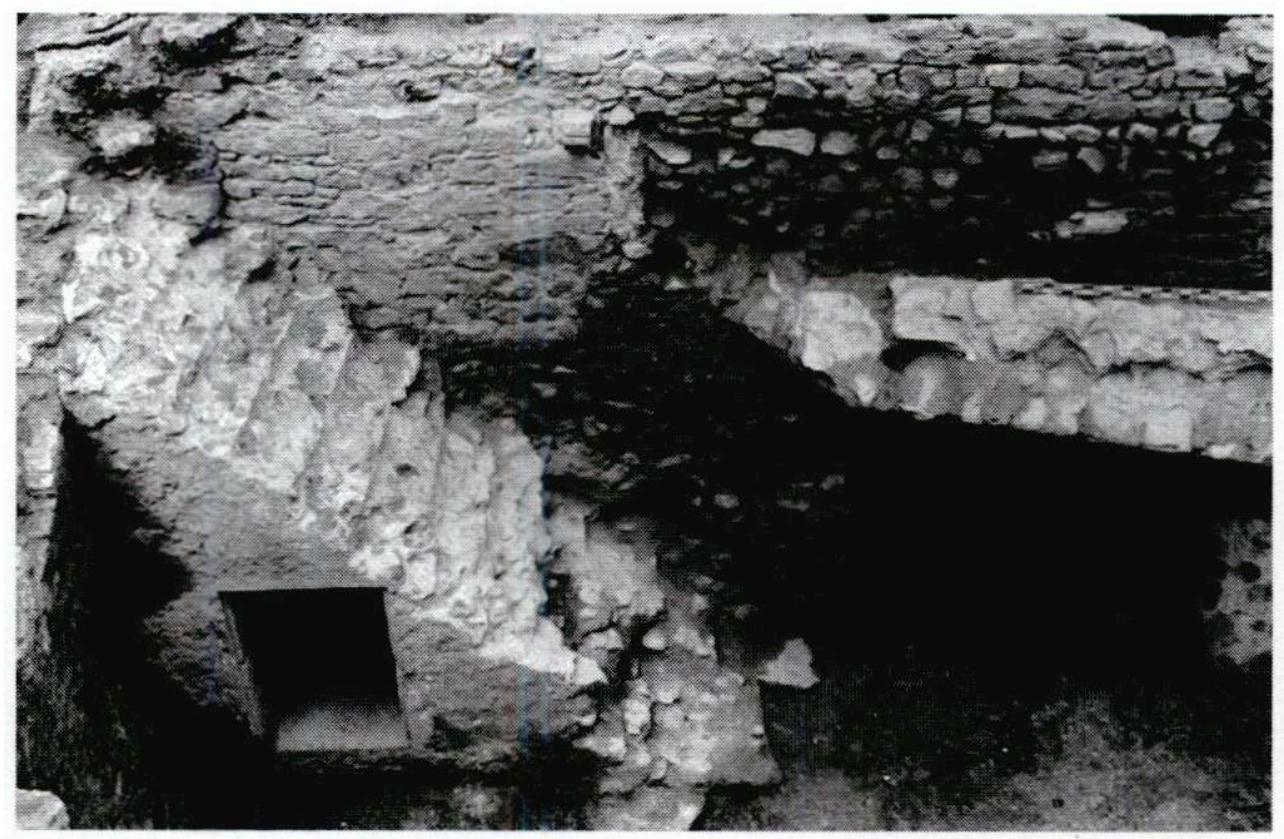

60 - Escalier, placard et départ des voûtes (cassées), parcelle D, rue Tramassac 


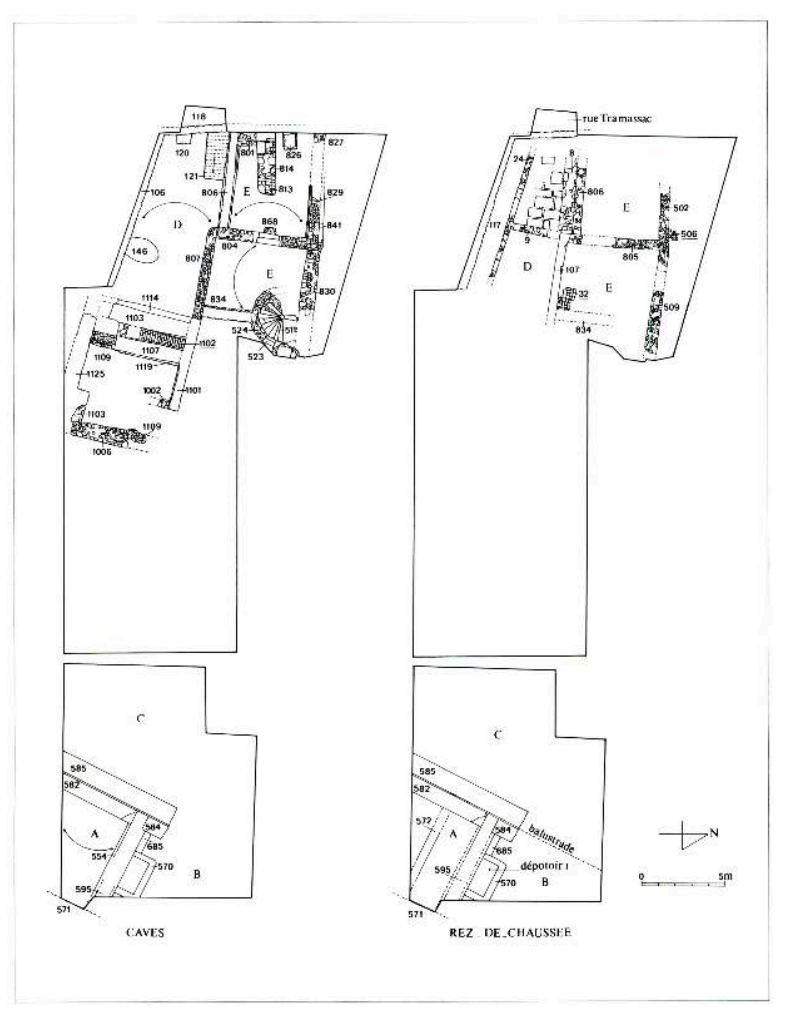

61- Plan des vestiges de la période 18, caves et rez de chaussée

"Estant comparu au Consulat Mesieurs Antoine Galliad (...) et Pierre Curnillon. notaire royal lesquels ont requis que mesure et allignement leur fussent donner pour un devons de maison qu'ils désirent reconstruire à neuf scize sur la rue du Gourguillon, après que les dits Sieurs Crollier et Hunet ont este commis députés par le Consulat pour voir les lieux et sur le raport par eux fait de la manière dont la dite maison devra estre allignée en suivans lallignement général... Batissants sur ladite rue Gourguillon les requérants allignerons en ligne droite à prendre depuis le repaire fixe marquée sur lenchant et encognure de la Maison du Sieur du Soleil maistre chandelier tournant de ladite rue Gourguillon entrée pisse truye jusque à autre repaire marquée sur lenchant et encognure de la Maison du Sieur Baumont tournant en rue de la Pionière ledit repaire estant reculée de sept pouces, le tout à la charge du recullement qui eschera et d'avertir le Voyer de la ville lors du décombrement des terres dudit bâtiment pour estre transportée par les entrepreneurs dans les lieux qui leur seront indiqué comme lorsque la première assise de pierre sera encore posée affin que le rez de chaussée soit donné commil doit estre, à la charge de faire aposer des chenec (chenaux) de fer blanc pour la conduite des eaux pluviales et de ne faire poser des cadettes sur rue qui excèdent plus d'un pied et demy en largeur et à fleur de pavé et que sur le tour recollement sera fait par lesdits Sieurs ou par ceux qui seront par eux commis et députés pour estre les contreventions au présent acte."

(VI. 15, rue Tramassac. 31 décembre 1682. A.M.L. DD 36-156).

Des monnaies ont été retrouvées dans les niveaux d'occupation de la cave de la parcelle D; elles présentent une chronologie allant de la fin du XVIe s. (monnaie d'Henri IV, 1593) au milieu du XVIIIe s. (monnaie de Louis XV, 1740).

Le dépotoir situé entre la maison sur rue et la maison sur cour de la parcelle D n'est plus utilisé ; il est scellé par un sol en carreaux de terre cuite destiné à assainir cet endroit. Ce sol présente un léger pendage nord-sud et une incurvation afin de faciliter l'évacuation des eaux sales ou des eaux de pluie (fig. 62). 


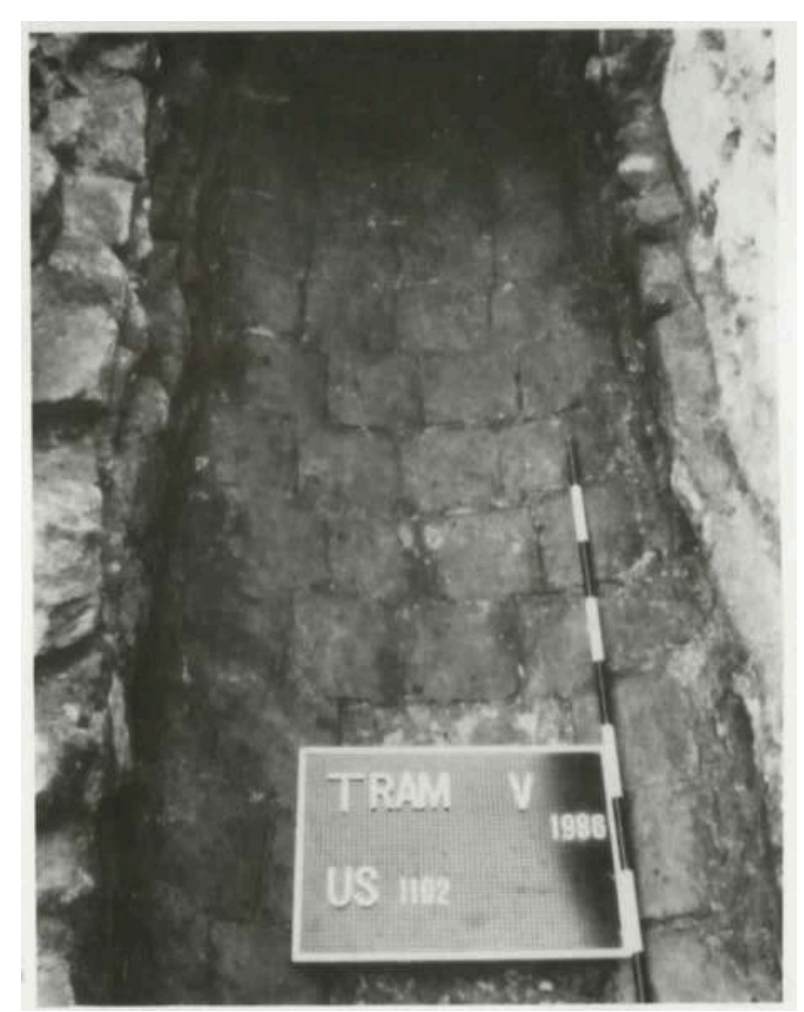

62 - Passage entre les maisons est et ouest, parcelle D

\section{Le tènement du 5 rue Carriès}

157 Les trois parcelles situées à l'angle des rues Tramassac et Carriès, ne semblent pas évoluer par rapport à la période précédente, et ce jusqu'en 1783. A cette date, un "permis de bâtir » demandé par le Sieur Portales, montre que ce dernier possède les parcelles ( 1 b et c) et (2) : “A Monsieur le juge Général seul voyer de la comté de Lyon, supplie le Sieur Portales Bourgeois de cette ville demeurant rue des trois maries, paroisse Sainte Croix, et a l'honneur de vous remontrer qu'il possède une petite maison scise rue des deux cousins dépendante de votre voierie, joignant du côté de soir une autre maison a lui appartenant et faisant l'angle septentrional et occidental des dittes rues. Son intention est de faire rebâtir ladite maison à la hauteur d'environ deux étages, il vous supplie de lui accorder les allignements necessaires à cet effet..." (A.D.R. 10.G.3814). L'état actuel de ces maisons est tel qu'il est décrit dans la réponse faite par le voyer. En effet, l'alignement sera exécuté et une façade "moderne" en retrait vient interrompre le rythme de la façade ancienne qui subsiste à l'ouest : “... permettons au Sieur Portales supliant de faire démolir et reconstruire la maison dont il s'agit sur la ligne de reculement tracé du dit plan; ledit reculement, suivant le dit plan a été reconnu être, savoir à la tête du mur joignant au couchant la grande maison du supliant de deux pieds, et à l'autre tête dudit mur au matin joignant le mur de Cloture du jardin du Doyenné de vingt un pouce..." (A.D.R. 10.G.3814). 


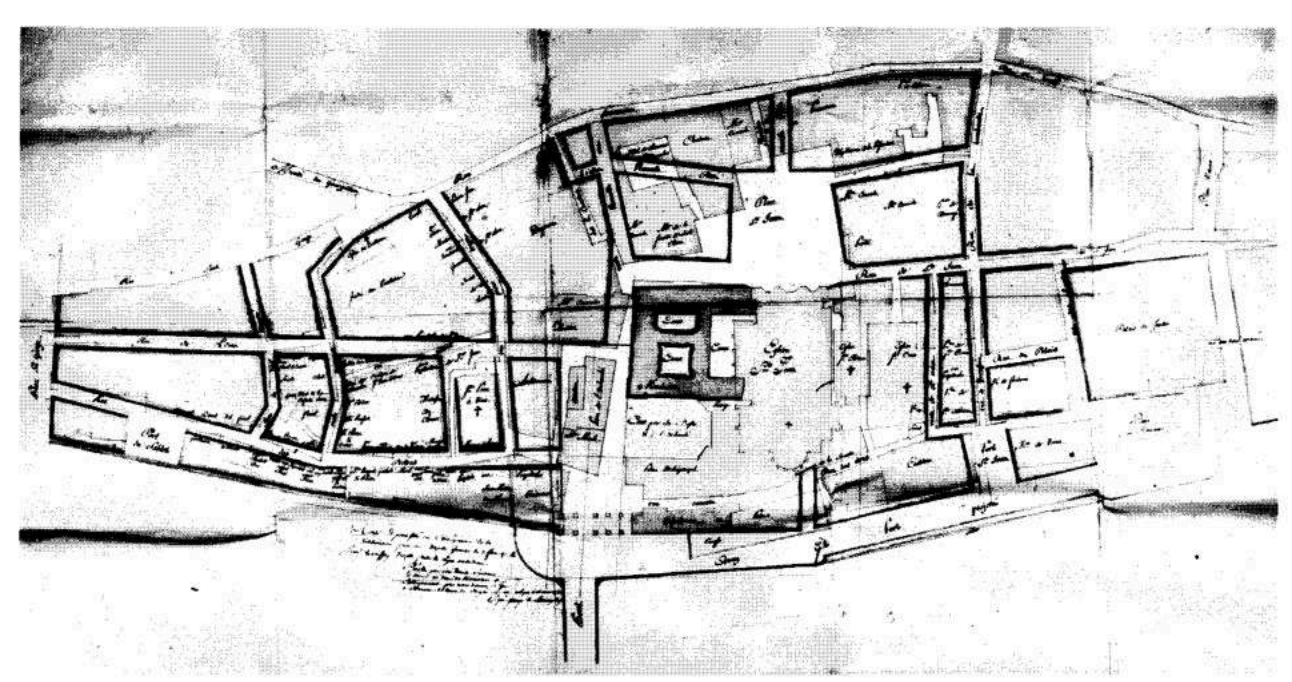

63 - Plan masse avec les alignements à exécuter - XVIIle s. (A.M.L. 1 S. 192)

158 Ce texte fait référence au plan d'alignement du quartier canonial de Saint-Jean dressé en 1770 (fig. 63), il confirme aussi la présence des jardins du doyen jusqu'au XVIIIe s.. La distribution intérieure même de ces maisons est reprise, avec l'aménagement d'un nouvel escalier contemporain de la reconstruction de ladite maison; l'accès à cet escalier, qui dessert les maisons est et ouest, se fait par une porte réaménagée dans la façade ancienne de la maison ouest.

\section{L'HÔTEL DU DOYENNÉ ET LES JARDINS}

Dans la partie est de la zone de fouille, le processus d'exhaussement se réalise progressivement. Le dépotoir est entièrement comblé et scellé par un remblai (US 604) dont le matériel est aux trois-quarts antique. Ce remblai scelle un remplissage dont les couches supérieures (US 678-679-685) sont datables du début du XVIe s.

160 L'exhaussement progressif des niveaux et l'abandon du dépotoir sont vraisemblablement liés à une transformation importante du mode d'occupation de cette partie du site. En effet, les jardins du centre de l'̂llot et l'hôtel du Doyenné sont dorénavant directement reliés. Le 21 février 1682 autorisation est donnée au doyen de faire démolir la muraille de la cour, jusqu'au terrain du côté du jardin, et de faire faire des grandes balustrades à la place de ladite muraille (A.D.R. 10.G.190.f ${ }^{\circ} 35$ et $10 \mathrm{G}$ 56.289). Cette description est confirmée par un texte de 1737 : "ledit jardin ou parterre est séparé de ladite cour par une balustrade de fer tendant de vent à bize; on monte de la cour en icelui par quelques marches pierre de taille". Les demandes d'ouverture, portes ou fenêtres, dans la muraille sont rares jusqu'en 1650 et accordées à titre exceptionnel avec de nombreuses servitudes; elles deviennent par la suite très nombreuses. La démolition de la muraille sur plusieurs mètres entre l'hôtel et le jardin en est un exemple.

161 Au début du XVIIIe s. des visites ou devis renseignent assez précisément sur le plan et l'aménagement de l'hôtel. Déjà en 1683, un texte laisse à penser que l'intérieur était assez luxueux ; le doyen, qui a des difficultés de paiement, offre de "laisser audit Hôtel les tableaux et boisage" qu'il a fait faire (A.D.R. 10 G 56. 292). D'après B. Plongeron, (Plongeron 1974) le doyen du chapitre recevait annuellement 12000 livres à la fin du XVIIe s. ; les autres dignitaires recevaient de 7000 à 8000 livres, les chanoines-comtes environ 3000 et enfin les perpétuels 800 à 900 livres. 
162 L'étude comparée du plan masse (A.M.L. 1.S. 192) (voir fig. 63), du cadastre et du Bref de Vente des Biens Nationaux (1791) permet d'établir la surface de la parcelle et ses contours. Par ailleurs, une visite de 1737 (A.D.R. 10.G.670.) très détaillée, confrontée avec plusieurs autres descriptions beaucoup plus vagues (devis de réparation pour le Doyenné, 1742, (A.D.R. 10.G.670) pièce 3 ; bail de location, 1747, (A.D.R. 10.G.670) pièce 4 ; visite de maisons canoniales, 1760, (A.D.R. 10.G.671) permet de se représenter l'organisation générale de l'ensemble.

163 Dans cette restitution (fig. 64), la surface des bâtiments à l'intérieur de la parcelle est supposée. Une partie du bâtiment 3 , qui correspond à la maison A décrite dès la période 14 , a pu être délimitée sur trois côtés (est, ouest et nord); cette pièce correspond à l'une des caves mentionnées "ayant un plancher au-dessus". De même la cour 1 a pu être repérée d'après les vestiges archéologiques (espace B). Mais l'emprise de la zone de fouille ne s'étend pas en fait au-delà de ces deux espaces, cave et cour. Pour le reste de l'hôtel du Doyenné, seules des hypothèses peuvent être émises d'après les textes d'archives, sans possibilité de les comparer à la fouille.

La visite de 1737 est le texte le plus riche en renseignements (voir encadré) :

- L'hôtel du Doyenné comprend (outre le bâtiment situé au nord de la rue Mourguet et correspondant au "Petit Doyenné") trois corps de bâtiment organisés autour d'une cour centrale et un quatrième au fond du jardin. L'entrée principale est située dans le bâtiment nord, face à la place Saint-Jean. L'accès aux étages des trois bâtiments se fait par un escalier en vis placé au sud-est de la grande cour. Le plan de type courant (maison avant et maison arrière séparées par une cour), adapté à un parcellaire laniéré repéré sur toute la rive droite de la Saône (par exemple rue Tramassac), n'apparaît donc pas pour l'hôtel du Doyenné, où se dessine d'après les reconstitutions, un plan en U. Les structures repérées en fouille correspondent à la cour centrale (l'espace B) et à une partie du bâtiment qui la ferme au sud (maison A).

165 Toutes les pièces mentionnées ont été replacées sur le schéma de reconstitution; leur surface est par contre totalement arbitraire. Cette demeure aristocratique comprend de nombreuses pièces d'habitation, plusieurs salles, une salle à manger, quatre chambres, "un petit appartement..."; une chapelle est aussi mentionnée dans le bâtiment 3. De nombreuses dépendances sont associées : cuisines (une au rez-de-chaussée et une autre au premier étage), remise, serre, bûcher, écurie, fenière...; l'ensemble est réparti sur trois niveaux (rez-de-chaussée, premier et deuxième étages); les combles sont occupés par des "grands greniers ou étendages et quelques galetas". 


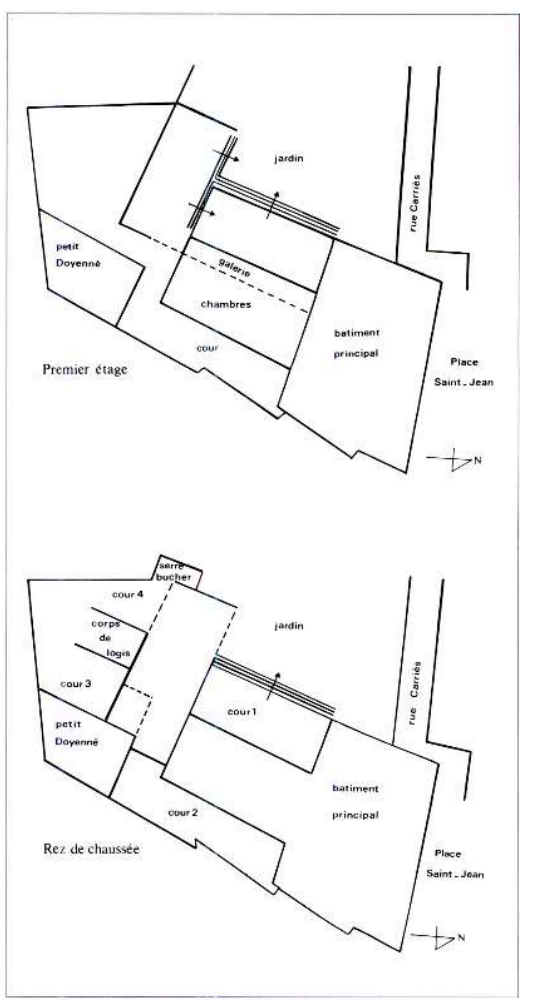

64 - Hôtel du Doyenné : proposition de restitution

Le texte donne des précisions sur le confort et la décoration: Dans la "grande cuisine" se trouvent "une grande cheminée de pierre de taille, four, potager et autres ajencements, le tout en bon état." Au premier étage une autre cuisine, plus petite, a elle aussi sa cheminée, son évier et son potager. Une autre cuisine est mentionnée, au deuxième étage, dans un petit appartement (bâtiment 3) "avec une couche et pierre d'évier qui dégorgent leurs eaux à travers du mur par un cornet de fer blanc et qui aboutissent dans un entonnoir..."

D'après le texte de 1742 (A.D.R. 10.G.670), la salle à manger est richement décorée à la mode du XVIIIe s.: "les murs sont revêtus de boisages en menuiseries dont les panneaux sont remplis de treize tableaux d'histoire peints à l'huile, le lambris au-dessus est un compartiment d'ouvrage de menuiserie en sculpture." Il mentionne également des latrines. Au premier étage, la galerie en arceaux de pierre de taille est "garnie de boisage et vitrage." Toujours au premier étage, au-dessus de la salle à manger, se trouve "une salle prenant jour sur la place Saint Jean par des grandes croisées à la française et garnies de leurs châssis papier et verre, et volets".

Ce texte de 1742, devis du sieur Roche architecte, mentionne à plusieurs reprises du "verre blanc d'Allemagne" pour les vitres des ouvertures. Les matériaux de construction utilisés sont la pierre de taille pour les cheminées, les escaliers et les encadrements de portes et fenêtres. Les revêtements muraux mentionnés sont principalement "l'enduit et plâtre" qui sont appliqués sur les pierres liées "avec des tuileaux et du bon mortier", enfin, les toitures dont les charpentes sont recouvertes avec des tuiles de Verdun.

EXTRAIT DU TEXTE CONCERNANT L'HOTEL DU DOYENNÉ

1737. A.D.R. 10.G.670

Nous, Hector de LEVY précepteur de l'Eglise et Messire Alexandre de MONTJOUVENT chanoine de ladite Eglise, aussi Comte de Lyon, sçavoir faisons que 
ce jourd'hui troisième septembre mil sept trente sept, commissaires députés de notre chapitre par acte du 28 juin dernier au sujet de la visite, rapport et description de la maison et hôtel du Doyenné être faite par (...)

Les battimens consistent en quatre corps de logis, l'un à l'entrée faisant face sur la place Saint Jean du côté bize, le second en retour d'équerre au long d'une grande cour et jardin du côté de soir, et du côté de matin, sur une cour dépendante de l'hôtel du doyenné sur la cour dépendant de la maison canonialle de Monsieur le Comte de Chemé ; le troisième corps de bâtiment fait face sur ladite cour du côté de bize et se retourne d'équerre sur le susdit ; le quatrième corps de bâtiment est en face de soir sur un bosquet étant au fonds dudit jardin. Tout lesquels bâtiments nous avons visitté et examiné : premièrement dans le rez-de-chaussée qui consiste, sçavoir : dans le premier corps de logis, une remise sur rue en face de bize, laquelle nous avons trouvé en bon état, la principalle porte d'entrée dudit hôtel est pareillement en bon état, à la réserve que le mur au long de l'entrée du côté de matin est en partie écorchée, l'enduit et plâtre étant tombé d'environ une toize carrée. Dans la même entrée, aux murs des côtés de bize et soir, l'enduit et plâtre sont pareillement tombés aussy d'une toize quarrée dans les deux endroits. Dans le même corps de bâtiments est une sale à manger dont les murs sont revêtus de boizages en menuiseries dont les panneaux sont remplis de treize tableaux d'histoire peints à l'huile, le lambris au-dessus est en compartiments d'ouvrage de menuiserie en sculpture. Ensuitte de ladite sale est une grande cuisine ayant une grande cheminée de pierre de taille, four, potager et autres ajancements, le tout en bon état. A l'angle de matin et vent de la cour est un grand escalier en vis pierre de taille qui sert pour monter au 1er et 2ème étage, à côté duquel est une porte qui conduit dans une cour par laquelle on descend dans deux grandes caves, l'une étant vout-tée en maçonnerie, et l'autre ayant un plancher au-dessus; elles sont sous le troisième corps de logis dont on a parlé. De ladite cour, on passe à une troisième du côté de vent, de laquelle on entre dans une grande écurie du côté de soir, au-dessus de laquelle est une grande fenière; quelques aix du plancher de ladite écurie sont rompues ainsi que le bas de la fermeture de la porte par laquelle on y entre; dans lesdites deux cours sont environ trois ou quatre tombereaux de terre, platras et tuileaux qui les embarrassent. Sur le quatrième corps de logis, toujours au rez-de-chaussée, est une serre à former des orangers et un bucher attenant divisé en deux au moyen d'une cloizon planches, tendant de matin à soir, le tout étant en assez bon état. Nous sommes montés par le susdit escalier au premier étage, et par iceluy sommes entrés sur une galerie en arceaux pierre de taille garnie de boizage et vitrage au fonds de laquelle du côté de bize est une porte par laquelle on entre dans une sale prenant jour sur la place Saint Jean par des grandes croizées à la françoyse garnie de leurs chassis papier et verre, et volets, le tout en bon état à la réserve d'un traversier ou renvoi d'eau qui est pourri et rompu. A côté de ladite sale du côté de matin est une chambre ayant vue sur ladite place ; et au bout de lad. salle, du côté de soir est un escalier en marche pierre de taille qui lui sert de dégagement ; à côté d'iceluy, du côté de vent, est un petit cabinet vitré ayant vue sur la cour et sur ledit jardin, et un second cabinet au bout duquel est un escalier dérobé qui aboutit sur la rue des deux Couzins; tout ce que dessus est en bon état, à la réserve d'une corruptin de l'angle des murs en face de bize et soir, laquelle règne du haut en bas : il parait qu'elle est depuis très longues années dans cet état et que lesdits murs ne suplombant point, cela est hors de 
péril ; cependant pour en ôter la déffectuosité, il conviendra la piquer, remailler et plâtrir du haut en bas.

$\mathrm{Au}$ long de lad. galerie sont trois chambres qui prennent leurs entrées par icelle et ont leurs jours sur la maison canonialle de Monsieur le Comte de Chemé : elles sont touttes en bon état. Au bout d'icelle, du côté du vent, est une petite pièce servant de cuisine : elle a sa cheminée, son évier et potager, le tout en bon état. Sur le troisième corps de logis est une grande sale basse élevée seulement de quelques marches au-dessus du rets-de-chaussée ; elle est accompagnée de quelques cabinets qui prennent le jour sur lad. cour et jardin de bize ; au-dessus desquels cabinets est un petit apartement qui prend aussy les jours du côtés de bize par lad. cour et jardin, tout lequel dessus nous a paru en bon état. A l'angle de vent et soir de lad. grande sale est un perron par lequel on entre dans une chambre étant dans le susdit corps de logis ; de ladite chambre, on entre de plein pied dans une seconde prenant jour du côté de matin sur la maison canoniale de Monsieur le Comte de Chemé, le tout en assez bon état. Au dit premier étage est aussi une chapelle prenant jour des côtés de matin et soir, laquelle est en bon état et descemment or une couche et pierre d'évier qui dégorgent leurs eaux à travers $\mathrm{du}$ mur par un cornet de fer blanc et qui aboutissent dans un entonnoir lequel est plein d'ordures, de cendre et de sable en sorte que les eaux n'ayant pas leurs vuidanges, elles purissent le couvert qui au-dessous (lequel il faudra repasser à tranchée ouverte, et changer les bois pourris) ; le surplus desd. petits appartemnts est en bon état. Sur le troisième et quatrième corps de logis dud. deuxième étage sont trois grands greniers ou étandages et quelques galetas. Au fond dud. jardin et bosquet est un petit pavillon couvert en forme de dôme qui est en bon état. A l'angle de bize et soir de lad. cour est un puits à eau claire ; led. jardin ou parterre est séparée de lad. cour par une balustrade de fer tendant de vent à bise ; on monte de la cour en iceluy par quelques marches pierre de taille.

Tous les couverts desd. bâtiments sont en thuiles creuses : les uns paraissent en bon état et aux autres parties des thuiles sont dérangées : il conviendra de les repasser et remanier, tout principalement du corps de logis du côté de bize étant sur l'entrée dud. hôtel, lequel il conviendra de repasser à tranchée ouverte et d'y fournir les thuiles qui pourront y manquer ; au mur de face du corps de logis depuis l'entrée en tendant à la rue des Deux Cousins, il y a une partie du mur de face dant l'enduit et le plâtre sont tombés, les joints même décharnés : il faudra le remailler avec des thuileaux et du bon mortier de chaux et de sable après avoir nettoyé et ratissé le mortier corrompu et qui tombent en farine ; après quoi on l'enduira et pla-trera, ce qui fera environ six toises quarrés ; et au surplus seront remaillés tous les trous qui se trouveront au mur dud. hôtel ainsi que les carreaux de verre qui se trouveront rompus(...)

\section{L'ÉPOQUE CONTEMPORAINE}

L'enceinte avait certes déjà perdu son allure de forteresse, mais en 1791 la distinction "extra muros" et "intra-muros" disparaît totalement avec la vente des propriétés de l'Eglise comme Biens Nationaux. Tous les bâtiments vont peu à peu être intégrés au reste de la ville et cet ensemble que formait le quartier canonial va être l'objet d'une urbanisation intensive. 


\section{L'HÔTEL DU DOYENNÉ APRES SA VENTE COMME BIEN NATIONAL (PÉRIODE 19)}

170 Le 28 mai 1791, l'hôtel du Doyenné est vendu comme Bien National (Bref de vente;

A.D.R. 1. Q.327) :

"On fait savoir que le vingt huit may mil sept cent quatre vingt onze, à deux heures de relevée et suivantes, il sera procédé à la vente, au plus offrant et dernier enchérisseur d'une maison ditte Le Doyenné, jardins et dépendances, appartenant à la Nation ci devant possédé par le Chapitre de Saint Jean dont la désignation suit.

Maisons et jardin dits du Doyenné ci devant possédé par le Chapitre de Saint Jean ayant environ vingt mille pieds carrés de superficie.

Confinés au Nord par la rue de l'Evéché et la rue des Deux Cousins sur une longueur de cent soixante trois pieds, au midy par la rue Pisse Truie sur une longueur de soixante et quinze pieds, à l'occident par les maisons Portales, Rival, et Sainte Luce, et à l'orient par deux maisons à la Nation l'une portant le $n^{\circ} 35$ et l'autre le n ${ }^{\circ} 37$ ditte l'Archidiaconé.

La partie de Bâtiment sus confinée par la rue de l'Evéché porte le n 34 . Elle comprend, à retz-de-chaussée des cuisines, offices, leurs dépendances, bûcher, deux escaliers, dont l'un est à limons suspendu, remises, une grande cour pavée en carreaux de pierres, et entourée d'un portique, un jardin fermé par une grille en fer et une pièce d'eau ou citerne dans ledit jardin.

Le premier étage forme quinze pièces dont douze à cheminée et plusieurs cabinets; le second étage forme plusieurs chambres de maîtres et domestiques, cabinets et greniers.

Sous partie du rez-de-chaussée sont des caves.

Les constructions sur la rue Pisse Truie forme du côté d'occident, une écurie, fenil, cour et dépendances, du côté de midy, un bâtiment en location portant le $n^{\circ} 40$ et qui est composé de caves, retz-de-chaussée et deux étages au dessus.

L'adjudicataire ne jouira que précairement des vues, croisées, jours et chuttes d'eau, pris par les maisons sus désignées sur les deux autres maisons à la Nation qui les confinent côté d'orient..."

171 De nombreuses modifications sont réalisées après cette vente.

La cave est voûtée (US 569) et un mur de cloison (US 572) est construit dessus. La porte donnant accès à la cour depuis la cave a été bouchée par un blocage de maçonnerie dans lequel est aménagé un soupirail. Enfin, le caisson rectangulaire située en avant de la même porte est utilisé comme dépotoir (fig. 65). La présence de cet aménagement installé depuis l'intérieur, non parementé à l'extérieur et situé dans la cour devant la porte, est difficilement explicable. De petite dimension, il a pu être installé dans la cour à la sortie de la pièce pour y ménager un accès (escalier ou échelle), à une période intermédiaire de l'exhaussement du sol comme l'attestent des illustrations anciennes (fig. 66). 


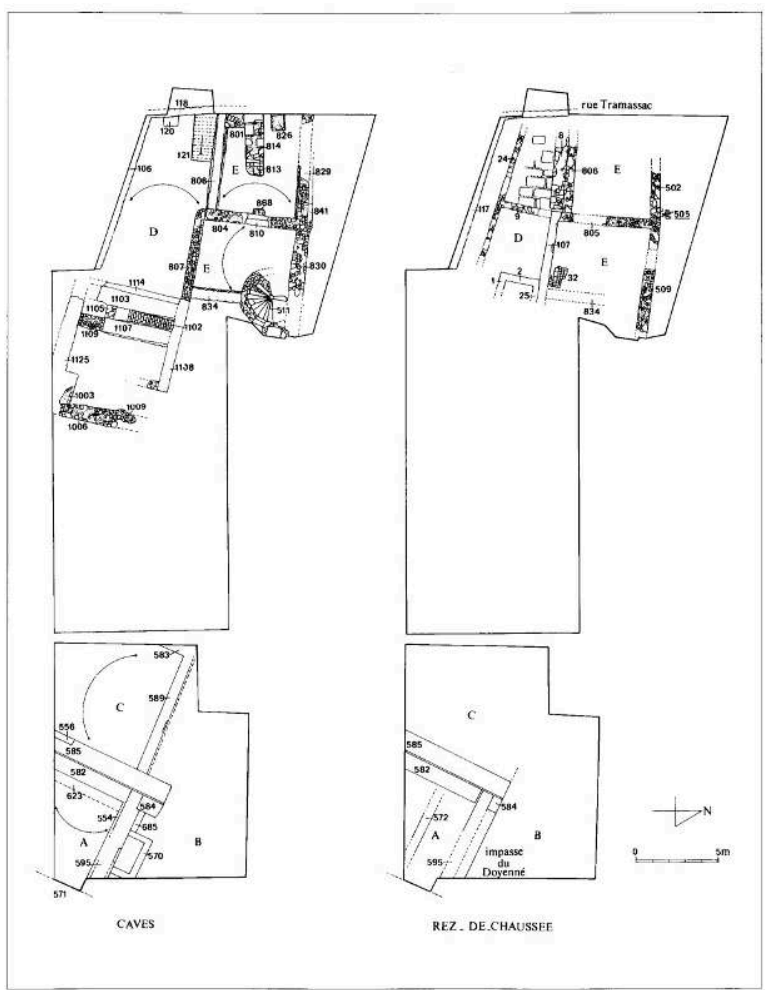

65 - Plan des vestiges de la période 19, caves et rez de chaussée

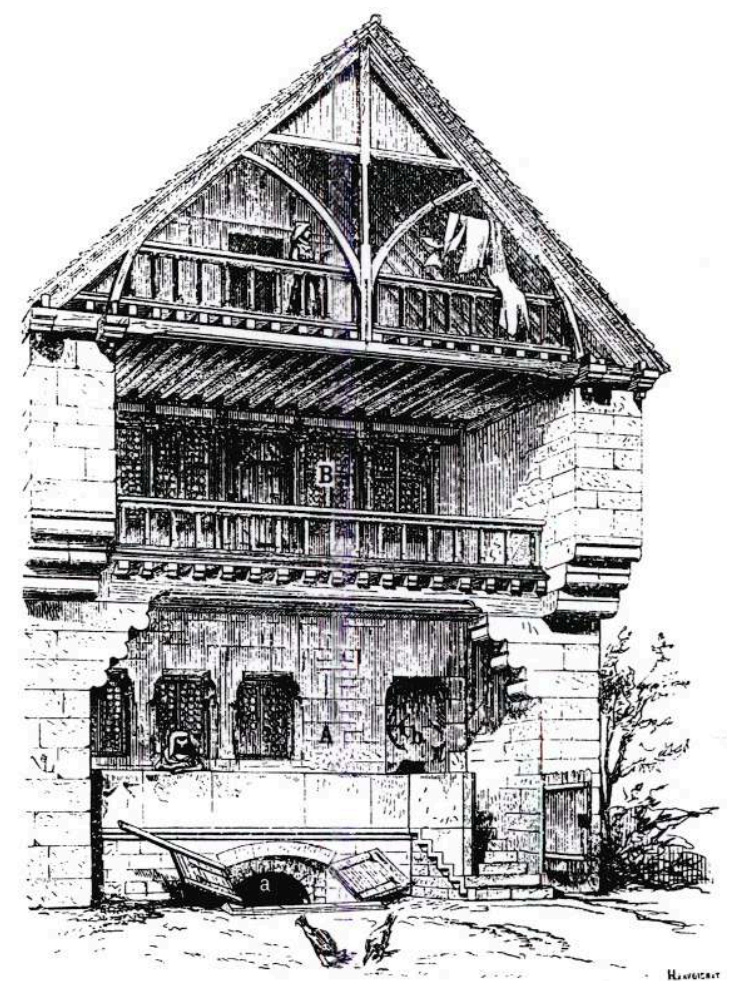

66 - Maison avec un accès à la cave aménagé après exhaussement du sol

Cette structure contenait une grande quantité de carreaux de poêle : en faïence blanche à décor bleu correspondant à une production lyonnaise du XVIIIe s. (US 602) et en faïence blanche du XIXe s. avec la base d'un poêle de forme arrondie (US 597). 
174 Un ensemble de verrerie du XIXe s. a aussi été découvert dans ce dépotoir, comprenant de nombreux verres à jambe pleine, dite "balustre" et des gobelets décorés de facettes en relief; ces verres sont transparents, d'une épaisseur de 3 à $4 \mathrm{~mm}$. Parmi les autres formes en verre, on trouve des bouteilles à vin et à champagne, une bonbonne de grande contenance, ainsi que divers petits objets, une salière-poivrière en verre transparent moulé, décorée de feuilles et de volutes, un bouton de porte moulé, transparent, un encrier moulé, décoré de lignes parallèles en relief, et une petite bouteille d'encre (fig. 67).

Plusieurs centaines de tuiles vernissées noires ou vertes ont encore été extraites de ce même dépotoir indiquant que ce dernier a dû être comblé lors de travaux exécutés dans la maison du Doyenné.

176 A l'ouest de la cave, là où étaient les jardins du Doyenné, une maison est construite, dont le niveau de cave a été mis au jour. Le mur s'appuie sur l'ancien mur d'enceinte qui est réutilisé. Un soupirail est installé dans le mur nord; ce dernier est situé dans le même alignement que celui de l'hôtel du Doyenné. L'espace situé devant ces deux bâtiments, au nord, n'est toujours pas bâti comme en témoigne la présence des deux soupiraux. La parcelle de l'ancien hôtel du Doyenné va être entièrement restructurée à la suite de la percée de la rue du Doyenné : la partie est de la parcelle est préemptée par la Ville en 1825 (fig. 68).

177 La partie est des bâtiments de l'hôtel du Doyenné se trouve donc sous la rue du même nom. A l'intérieur du nouvel îlot, la parcelle est restructurée en immeuble de rapport. la rue du Doyenné. Vers 1846, le propriétaire de cette impasse propose de la céder à la Ville (A.M.L. Série 0. Impasse du Doyenné) :

"Rapport à Monsieur le Maire,

L'impasse à l'ouest de la rue du Doyenné située entre la rue des Deux Cousins, et la rue Saint Pierre le Vieux appartient totalement au Sieur Mory.

Sa largeur est de six mètres et sa longueur de 40 mètres environ : son extrémité orientale est située à peu près en face d'une nouvelle rue protégée ainsi qu'il résulte du plan ci-annexé.

Le Sieur Mory propose de céder cette impasse à la Ville sous les conditions énnoncées dans une lettre ci-jointe en date du 8 septembre 1845. Ces conditions sont très peu onéreuses pour la Ville, attendu qu'il faudrait pourvoir qu'à l'entretien du pavé et d'un réverbère.....

Par conséquent le soussigné est d'avis qu'il y a lieu à accepter la proposition du sieur Mory.

Lyon, le 4 février 1846. signé Cafrini." 


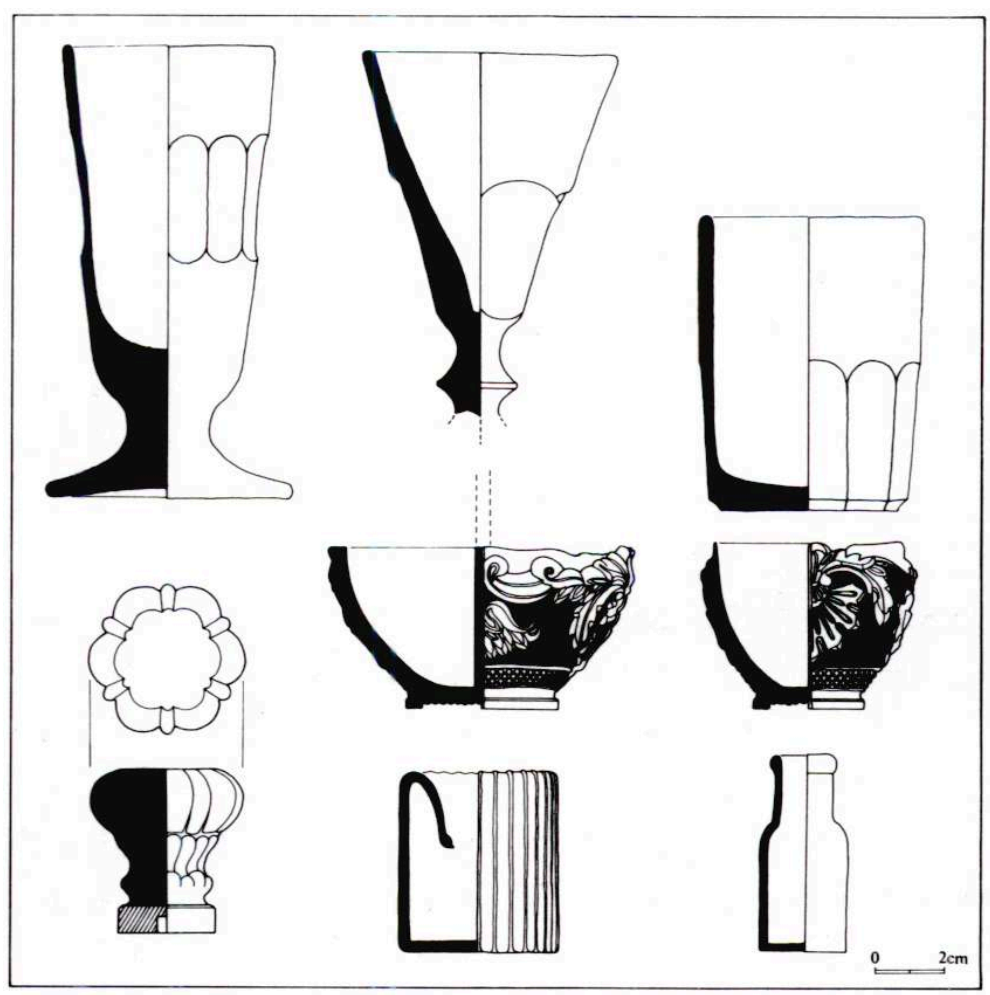

67 - Verres

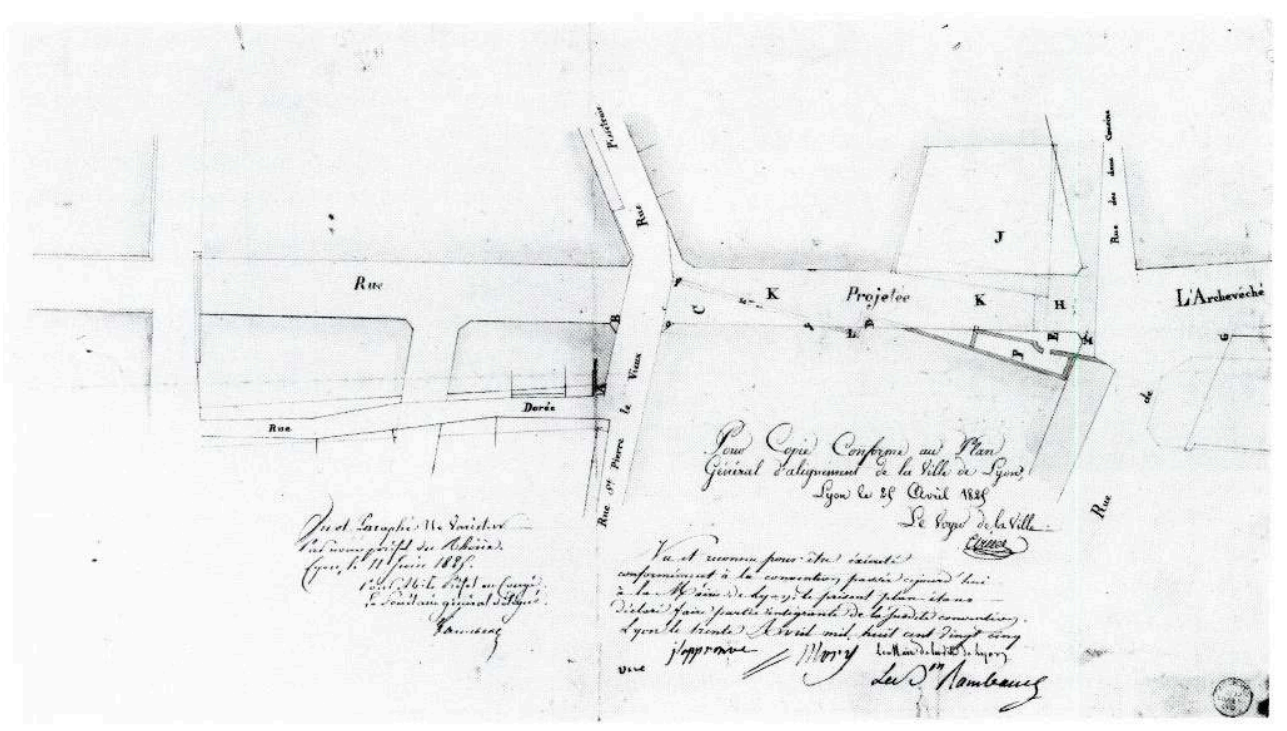

68 - Projet d'alignement pour la percée de la rue du Doyenné 1829 (A.M.L. 3 S. 313)

Rue Jean Carriès, des immeubles sont construits à l'emplacement des jardins du Doyenné et de la partie nord de l'hôtel du Doyenné, qui respectent l'alignement donné au Sieur Portales par le voyer en 1783. De même, l'autre côté de la rue Carriès subit un alignement, ce qui élargit celle-ci considérablement.

Jusqu'au XVIIIe s., seule la rue Tramassac permettait une communication entre les quartiers Saint-Georges (sud) et Saint-Paul (nord), de part et d'autre du quartier canonial. L'élargissement de la rue Carriès correspond déjà à une volonté d'aménager un système de voirie sclérosé par la présence d'un quartier fermé; cette rue permet 
l'accès au nouveau pont de Saône par la rue de l'Archevêché et à la montée du Chemin Neuf percée par les Protestants en 1562. Cette politique se poursuit et en 1866 la percée de l'avenue Adolphe Max vient définitivement transformer le paysage à l'est de l'îlot. Les façades du début du siècle, sur la rue du Doyenné, se trouvent alors face à une perspective dégagée jusqu'à la Saône.

Rue Tramassac, les deux parcelles étudiées sont occupées sans discontinuité jusqu'en 1874. Cette occupation est attestée par des niveaux de sols (dalles, tommettes...) et une amélioration des installations d'hygiène (fosse septique, canalisation, égout...). L'étude numismatique confirme une occupation des niveaux de caves des parcelles $\mathrm{D}$ et $\mathrm{E}$ jusque dans la seconde moitié du XIXe s. (monnaies de Napoléon III).

\section{LA RESTRUCTURATION DE L'ÎLOT AU XIXe S. (PÉRIODE 20)}

A la fin du XIXe s., un véritable curage est réalisé, depuis la rue Tramassac jusqu'à l'ancienne parcelle du Doyenné, traversant ainsi tout l'îlot.

En 1874, la liste des expropriations pour cause d'utilité publique est publiée. Les immeubles sont détruits et les caves remblayées avec les matériaux de la démolition (parcelles D et E).

Le 8 août 1878, le funiculaire reliant Saint-Jean à Saint-Just est inauguré. La gare est bâtie au nord-est de l'îlot; une cave est construite au centre de l'îlot dans les anciens jardins du Doyenné, pour y installer les pompes Jandin et autres machines utilisées pour faire fonctionner le funiculaire (fig. 69).

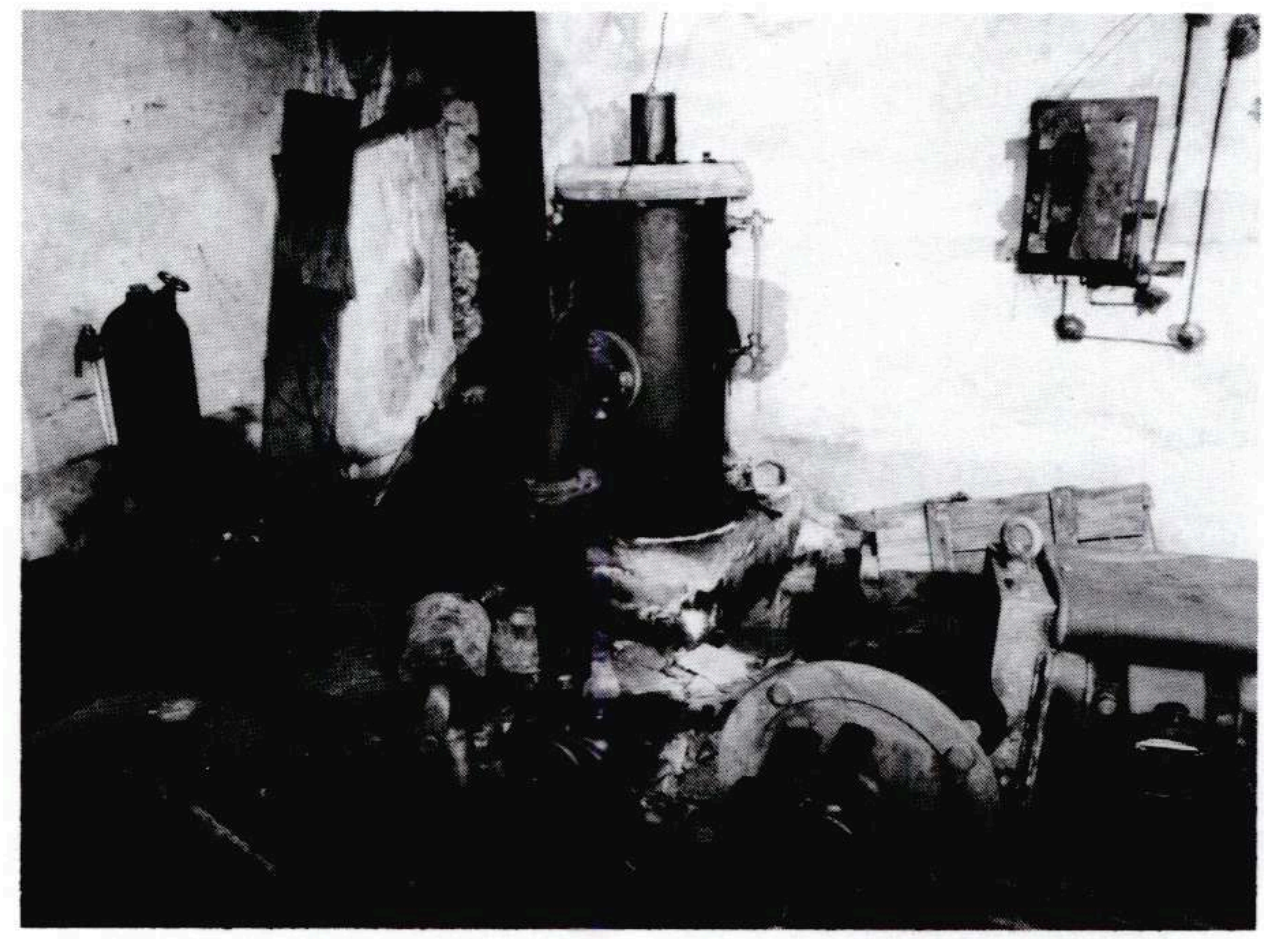

69 - Pompe Jandin

$185 \mathrm{Au}$ XXe s., une deuxième ligne de funiculaire reliant Saint-Jean à Fourvière est créée, occasionnant la démolition d'autres immeubles rue Tramassac (la parcelle "la" est démolie) ; la gare est modifiée. 
Par ailleurs, des immeubles sont reconstruits le long de la rue du Doyenné, en 1843 au nord de l'impasse du Doyenné et en 1845 au sud de cette même impasse (fig. 70).

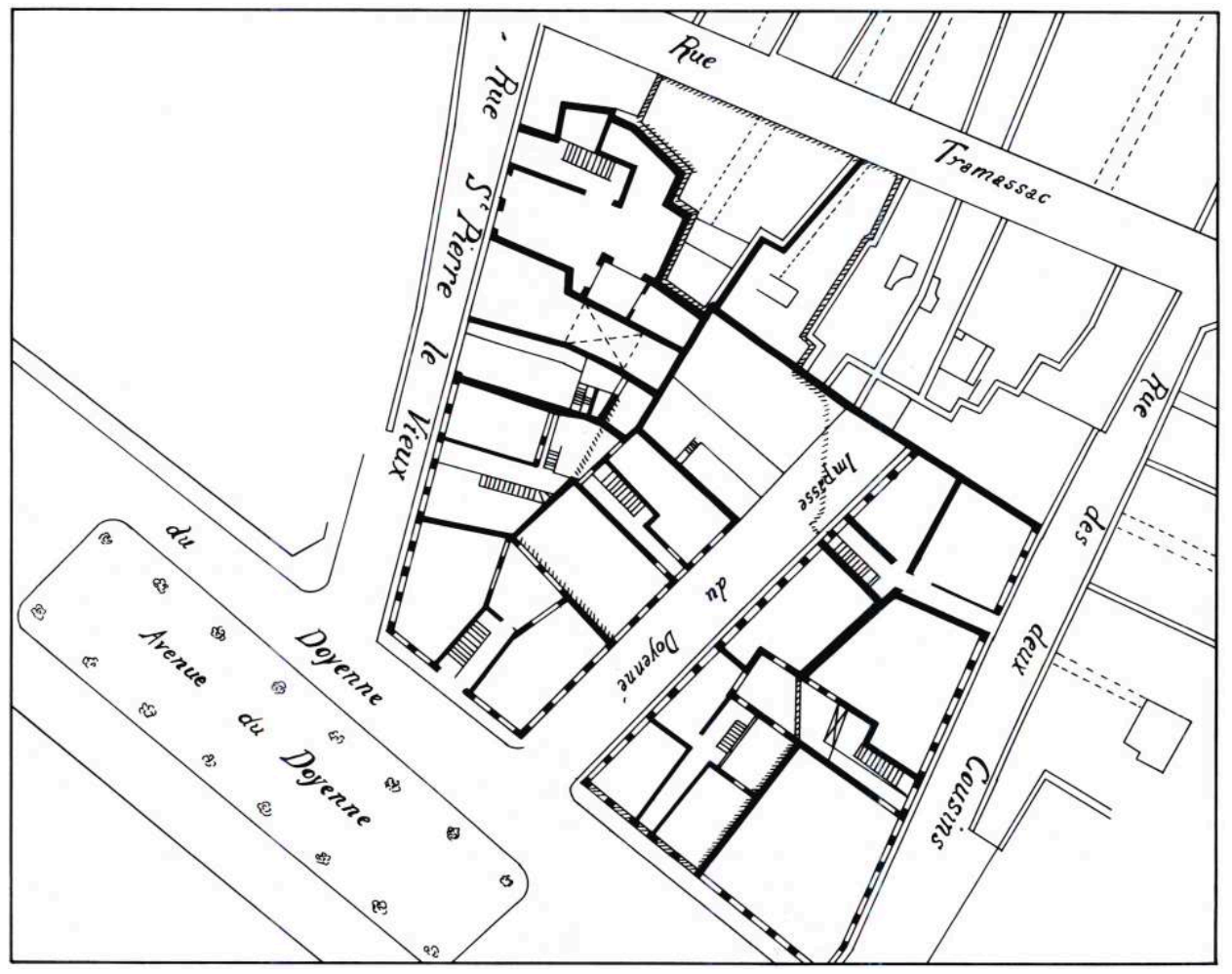

70 - Etat du parcellaire à la fin de la période 19. 7 juillet 1875 (A.M.L. serie 0)

\section{NOTES}

5. Les datations sont données avec réserves, compte tenu de l'absence de références régionales. 


\title{
Troisième partie. Éléments de culture matérielle
}

\author{
Jean-Michel Poisson et Bruno Claret
}

Les auteurs remercient M. Dhenin, conservateur au Cabinet des Médailles de la Bibliothèque Nationale pour son aide.

\section{LA CÉRAMIQUE GALLO-ROMAINE}

\section{Avertissement}

1 La présentation du mobilier céramique mis au jour lors des opérations archéologiques de lîlot Tramassac et de la rue Carriès ne constitue nullement une étude exhaustive du mobilier issu des niveaux de l'occupation antique ${ }^{6}$. Une telle présentation aurait réclamé un temps d'étude beaucoup plus long afin d'arriver à une synthèse de l'ensemble des résultats portant sur la reconnaissance des formes et leur évolution au sein de chacune des productions concernées. En effet, le temps d'étude réservé au mobilier est souvent amputé du long travail de l'inventaire qui, outre l'identification de la céramique, inclut le lavage, le marquage et le conditionnement des tessons. Ces exigences compromettaient l'analyse détaillée du mobilier. Aussi, nous avons adopté le parti d'une présentation des éléments de datation fournis par la céramique des principales périodes de l'occupation antique : de fait, n'ont été sélectionnés que les lots de couches qui, par leur position stratigraphique, ont fait l'objet d'un commentaire archéologique et dont la datation avancée nécessitait une justification. Nous n'avons pas tenu compte (et cela constitue notre deuxième option), de la nature du gisement d'origine des céramiques (remblais, préparation de sols...) que nous avons regroupées à l'intérieur de chaque séquence chronologique. Ceci n'est pas sans conséquence sur la composition de ces lots et peut donc influer sur la fréquence des types de production. 


\begin{tabular}{|l|c|c|c|c|c|c|c|}
\hline & & \multicolumn{2}{|c}{ Fines } & \multicolumn{2}{c|}{ Communes } & \multicolumn{2}{c|}{ Amphores } \\
\hline Périodes & Phases & Vases & Tessons & Vases & Tessons & Vases & Tessons \\
\hline Période 2 & Installation de la maison & 48 & 116 & 158 & 295 & 158 & 252 \\
\hline Période 2 & Occupation & 5 & 6 & 7 & 8 & 8 & 13 \\
\hline Période 2 & Démolition & 70 & 83 & 318 & 349 & 323 & 376 \\
\hline Période 3 & Installation de la voie 278 & 17 & 20 & 210 & 218 & 147 & 164 \\
\hline Période 3 & Fréquentation de la voie & 45 & 50 & 119 & 130 & 71 & 75 \\
\hline Période 3 & Occupation du IVe & 139 & 160 & 296 & 306 & 135 & 145 \\
\hline Période 4 & Installation de la voie 272 & 65 & 69 & 84 & 106 & 255 & 281 \\
\hline Période 4 & Le dépotoir d'os & 513 & 534 & 999 & 1009 & 638 & 648 \\
\hline Période 4 & Remblai du sol 707 & 28 & 28 & 64 & 64 & 97 & 104 \\
\hline Période 4 & Les structures palatiales & 89 & 93 & 213 & 225 & 109 & 115 \\
\hline Période 5 & La dernière occupation & 35 & 36 & 159 & 159 & 40 & 40 \\
\hline Période 5 & Niveau de la voie 231 & 25 & 25 & 92 & 93 & 32 & 33 \\
\hline Période 6 & Démolition-incendie & 247 & 253 & 522 & 540 & 493 & 509 \\
\hline TOTAL & & 1326 & 1473 & 3241 & 3502 & 2506 & 2755 \\
\hline
\end{tabular}

71 - Répartition du mobilier céramique gallo-romain de lî̂lot Tramassac et de la rue Carriès

2 Les différentes propositions chronologiques reposent sur la reconnaissance des formes les plus fréquentes, donc facilement identifiables, et l'évolution de chacun des types de production (sigillée gauloise, claire $\mathrm{B}$, africaines $\mathrm{C}$ ou $\mathrm{D}$, métallescente...) au sein d'une même catégorie (fines, communes ou amphores). Pour les périodes tardives, cette évolution est sensiblement troublée par l'importance du mobilier résiduel, synonyme de remaniements successifs, rendant tout bilan aléatoire. Souvent, le recours à la position stratigraphique de la couche à laquelle appartient le lot examiné est venu compenser les insuffisances du mobilier céramique exhumé (par exemple pour les périodes 5 et 6 ).

3 La céramique issue des lots analysés réunit $63 \%$ de l'ensemble inventorié, ce qui correspond à 7073 vases $^{7}$ soit 7730 tessons, toutes productions confondues. Cette étude ne propose par conséquent qu'une vue partielle de la céramique mise au jour dans les secteurs étudiés et ne repose que sur un premier examen du mobilier (fig. 71).

4 L'analyse céramique relève de différentes approches. En effet, la céramique commune, qu'il est pour le moment quelque peu prématuré de vouloir cerner, surtout dans des contextes tardifs encore peu étudiés, n'a pu offrir les mêmes avantages que la vaisselle fine qui bénéficie d'un meilleur degré de connaissance. Malgré son importance quantitative (près de $46 \%$ ), le vaisselier culinaire est donc peu intervenu pour moduler la chronologie. Parmi le mobilier amphorique, l'observation à l'œil nu de la facture de la pâte a permis de distinguer des groupes de production présentant des caractéristiques homogènes (produits africains, gaulois, certains types hispaniques ou orientaux). Mais des problèmes d'identification, et donc de provenance, restent toutefois posés, surtout dans les périodes tardives.

5 La céramique gallo-romaine exhumée témoigne d'une occupation s'échelonnant du IIIe s. à la fin du VIe s., terminus considéré, du point de vue technologique et économique, comme le seuil du Moyen Age ${ }^{8}$. Ce mobilier s'inscrit donc en grande partie dans la période dite de l'Antiquité tardive. Or, l'un des caractères distinctifs actuels de la céramique de cette époque est la difficulté d'appréhender, du moins dans nos régions, toute évolution chronologique. Celle-ci est encore peu définie par manque d'éléments comparatifs publiés (Villedieu 1990). Si l'utilisation des indices monétaires reste assez aléatoire en raison de la longue circulation des types à l'époque tardive et ne constitue en définitive qu'un terminus post quem, l'existence de quelques rares fragments de 
produits importés établit, parfois, des repères chronologiques précis, mais fragiles car peu abondants.

6 La quantité de céramique traitée et l'étirement chronologique nous ont autorisés à utiliser, parfois, l'argument a silentio : l'absence d'un type de production dans un lot céramique place la formation de celui-ci avant l'apparition de ce type.

7 Ne disposant pas des comparaisons aussi nombreuses et détaillées que fournissent les périodes plus anciennes, il fallut s'en remettre à la présence des deux types de production qui jalonnent, dans nos régions, l'Antiquité tardive: la céramique dite luisante ${ }^{9}$ et la sigillée paléochrétienne grise, appelée plus couramment céramique estampée grise ${ }^{10}$. Ces deux fossiles assurent ainsi une alternative aisée entre le début du IVe s., apparition de la céramique luisante ${ }^{11}$ et celui du Ve s. au cours duquel circule l'estampée grise ${ }^{12}$. Mais si les dates d'apparition de ces deux types de production sont généralement admises, leur durée d'utilisation et leur évolution ne semblent pas encore fixées.

8 Malgré les inévitables incertitudes de la stratigraphie des fouilles en milieu urbain dues, en grande partie, aux nécessités de délais des opérations de sauvetage et aux remaniements successifs durant les différentes phases de l'occupation, les regroupements complexes en séquences, phases et périodes opérés sur les chantier de l'îlot Tramassac et de la rue Carriès ont permis d'élaborer la trame d'une chronologie relative et d'aborder plus facilement l'approche du mobilier exhumé : ainsi, il ressort de cette analyse la simultanéité des productions de luisante et estampée grise tout au long du Ve s. ${ }^{13}$.

9 Mais le problème restait entier pour le VIe s. Il fallut, dans un premier temps, tenir compte d'une forme ponctuant le Haut Moyen Age, l'olla en commune grise dont la production débute au VIIe s. $^{14}$, puis essayer de trouver le prototype parmi les céramiques des niveaux antérieurs. Nous avons donc essayé de démêler le processus qui a présidé au développement de la lèvre en bandeau. L'examen plus particulier des céramiques communes grises et sombres, conjugué aux données de la stratigraphie a permis d'évoluer tout au long des Ve et VIe s. et d'avancer des datations qui restent toutefois prudentes.

\section{Méthode de présentation}

10 Nous avons appliqué à l'ensemble du mobilier un inventaire basé sur le nombre de vases estimé après collage. Cette évaluation varie selon la nature de la céramique (commune claire, amphores gauloises) ou du gisement d'origine (remblai d'installation, couche d'occupation...). Malgré l'approximation inévitable de ce type de comptage, cette méthode a été choisie afin de proposer une base quantitative. Celle-ci est souvent sousestimée bien que, reflétant les indices de fréquence des types concernés, elle permette de déduire les modalités de leur diffusion et donc l'image des principales tendances du mobilier (Ayala 1992, p. 296). Nous avons appliqué au mobilier de Tramassac-Carriès la méthode déjà mise en oeuvre lors du traitement des céramiques du chantier Adolphe Max (Villedieu 1990, p. 145 à 153). Cette méthode commune, privilégiant les associations de céramiques, permettra d'intéressantes confrontations tout au long de cette analyse.

11 Cependant, afin de ne pas alourdir cette présentation par une accumulation de données chiffrées, seuls les pourcentages des types de production évoqués apparaittront dans le 
commentaire. Un traitement statistique a servi à élaborer les courbes graphiques présentant la variation de fréquence des produits dont le commentaire est proposé en conclusion. Mentionnons déjà que la confrontation des données chiffrées a permis de constater, pour la période tardive, une différence minime entre le nombre de tessons et le nombre de vases. Cette différence souligne le caractère très fragmentaire de la céramique mise au jour sur les chantiers archéologiques de Tramassac et de Carriès, du fait de l'absence de véritable dépotoir d'époque gallo-romaine.

12 La définition de certains types de production nécessite quelques précisions. En céramique commune, dite de cuisine, le terme "sombre" est une appellation aisée qui devra sans aucun doute être révisée. Il regroupe une production hétéroclite, de cuisson oxydante (mode A), présentant une grande diversité de pâte (souvent riche en mica, orangé ou marron, parfois à gros dégraissants de quartz) et de couleurs certainement inhérentes à différents centres de production. Il est certain qu'une étude plus approfondie distinguera des groupes mieux définis par une analyse des pâtes, de la forme et de la destination des poteries. La mention "grise" regroupe, elle, des produits à pâte grise ou noire, à cuisson réductrice (mode B), grossière ou mieux épurée, à surface brute ou lissée et dont certains exemplaires tardifs portent, parfois, un décor incisé, peigné ou appliqué à la molette.

13 Ce que nous entendons par "amphores non identifiées" regroupe des fragments dépourvus de critères distinctifs et dont l'origine de fabrication reste à définir.

14 L'illustration a été limitée aux formes inédites et à celles présentant un profil ou un décor particulier. Les formes fréquentes de sigillée gauloise, de claire B et d'amphores, celles de céramique luisante (coupe carénée de type Lamboglia $1 / 3$ ou bol à décor d'ocelles Lamboglia 2/37 par exemple), ne seront pas reprises ici.

\section{LES AMÉNAGEMENTS DES BERGES DU BRAS DE SAÔNE (PÉRIODE}

1)

15 La faible quantité du matériel (5 fragments de panse en commune claire - 1 fragment de panse en commune grise) trouvé lors du dégagement des pieux d'un appontement sur la rive droite du bras de Saône rend toute datation impossible et en fait un document de peu d'intérêt. Les résultats des analyses dendrochronologiques (27 av. J.-C., 8 ap. J.C., 18 ap. J.-C.) ne peuvent être corroborés par le mobilier céramique.

\section{LES NIVEAUX DE REMPLISSAGE DU BRAS DE SAÔNE (PÉRIODE 2)}

Les céramiques prélevées dans les deux premières séquences de remplissage du bras de Saône sont à prendre avec précaution, car elles ne sont pas en liaison avec un système stratifié. Les séquences sédimentaires examinées en vue de l'analyse géomorphologique, et correspondant aux remplissages du bras de Saône opérés jusqu'au IIIe s., n'ont pas fait l'objet d'une fouille systématique. Le mobilier prélevé n'offre donc qu'un échantillonnage limité des céramiques contenues dans ces différents niveaux. Au regard des conditions particulièrement hasardeuses de la formation de ces lots, seule la fourchette chronologique, estimée après examen de quelques formes reconnues, sera proposée. 


\section{LES PREMIERS REMPLISSAGES}

17 Les fragments de gobelets à parois fines sablées ou à décor de pastilles, qu'il est possible de rapprocher des productions de l'atelier lyonnais de La Butte, (Grataloup 1988, type III, pl. XVI, no 2 à 4 , et type VII, pl. XVII, n 9) ainsi que les fragments de sigillée gauloise, (formes Drag. 24/25, Drag. 17 et Drag. 18/31), fournissent un terminus post quem précis : la première moitié du 1er s. ap. J.-C.

Cependant, la connexion du mobilier amphorique (formes Beltran-Lloris II et Dressel 28 d'origine hispanique) concourt à situer le lot durant le 1er s., époque de la plus grande diffusion de ces produits (Paunier 1981, p. 357, n 429 - $430 ;$ p. 357, n 432 à $434 ;$ p. 358, $\mathrm{n}^{\circ}$ 438). Toutefois, l'exclusivité de la forme $\mathrm{G} 4$ tendrait à placer ces premiers remplissages de bras de Saône à partir de l'époque flavienne, la commercialisation de cette forme d'amphore semblant prendre le pas, dès cette période, sur la forme $\mathrm{G} 5$ (Laubenheimer 1985, p. 390).

19 Parmi les céramiques communes, les deux fragments de mortiers à bord pendant, d'époque flavienne, ne contredisent pas cette datation (Desbat et alii, pl. IX, n ${ }^{\circ}$ ).

20 L'absence de claire B est un argument a silentio pour situer le terminus a quo des premiers remplissages à la fin du premier quart du IIe s., (Desbat 1988, p. 94). La formation de ce gisement a pu intervenir durant la deuxième moitié du Ier s., voire le début du siècle suivant.

\section{RÉTRÉCISSEMENT DU BRAS DE SAONE}

21 Le mobilier céramique se rapportant aux séquences sédimentaires du rétrécissement du bras de Saône offre un horizon chronologique postérieur à la période précédemment décrite. Si la présence de claire $B$ donne accès dorénavant au deuxième quart du Ile s., l'existence de fragments de gobelets rapprochés des productions métallescentes, qui apparaissent à Lyon dans la deuxième moitié du IIe s., affine cette datation (Desbat 1978, p. 43). Les quelques fragments d'amphores africaines, attestées dans nos régions à partir de la fin du IIe s, ceux d'une production orientale de forme Kapitan II (Keay 1984, p. 137), repérée à Ostia au début du IIIe s., et surtout l'absence de claire C, présente dans la phase suivante, limitent la formation de ces séquences sédimentaires du rétrécissement du bras de Saône au premier quart du IIIe s.

\section{LE COMBLEMENT DES CHENAUX RÉSIDUELS DE CRUE}

22 Les éléments de datation sont fournis par l'existence d'une assiette en claire B (forme Desbat 3), apparaissant à la fin du IIe s. et attestée à Lyon jusque dans la seconde moitié du IIIe s. (Desbat 1988, p. 96) ainsi que par un bord de claire C (forme Hayes 50) commercialisée en Gaule à partir du deuxième quart du IIIe s. (Carrandini et alii 1968, p. 34-36).

23 Ces niveaux de comblement des chenaux ont été également repérés dans la cave I du chantier de la rue Carriès. Parmi le mobilier prélevé se détachent un fragment d'africaine $C$ et un bord de claire B (forme Desbat 2/3). Le comblement des chenaux, commun aux chantiers de Tramassac et Carriès, a pu survenir aux alentours du milieu du IIIe s., l'absence de céramique luisante interdisant de placer cette phase au-delà de la fin du IIIe s. 


\section{LA MAISON AUX STUCS}

24 Cette installation constitue la plus ancienne phase d'occupation repérée lors de la fouille de la rue Carriès, phase d'occupation contemporaine de la dernière séquence de comblement du bras de Saône. Cette installation est concrétisée par une série de murs en briques crues, dont certains portaient encore un décor d'enduit peint, surmontés par une corniche en stuc (cf.: 2ème partie). Le mobilier céramique des différentes couches appartenant aux phases successives de la maison aux stucs constitue un ensemble clos du fait de l'effondrement des superstructures. La cohérence, l'abondance du mobilier et la position stratigraphique des couches étudiées ont permis d'élaborer l'évolution de cette habitation depuis ses remblais d'installation au début du IIIe s. jusqu'à ses niveaux de destruction survenue à la fin de ce siècle.

\section{REMBLAIS D'INSTALLATION DE LA MAISON}

Cet épais niveau, interprété comme les remblais d'installation de la maison aux stucs, a livré une quantité notable de céramiques (364 restes de vases, soit 663 tessons). La présence non négligeable de métallescente $(25 \%)$ et celle d'importation africaine (10,7 \% d'amphores, dont un fragment portant une inscription incisée (fig. $\left.72 \mathbf{n}^{\circ} \mathbf{1}\right)$ et 6,3\% de céramiques culinaires de formes Hayes 181, 196 et 197 - Hayes 1972, p. 201, 208 et 209) diffusées à partir de la fin du IIe s. offrent un terminus post quem précis. La présence appréciable de claire B (54,7\%), formes Desbat 3, 15, 50 et 53 (inscription incisée non identifiée) (fig. $72 \mathbf{n}^{\circ}$ 2), ainsi que celle de sigillée gauloise (21\%), en particulier une forme de Gaule du Centre Déchelette 72 (fig. $72 \mathbf{n}^{\circ}$ 3) placent l'installation de la "maison aux stucs" dans la première moitié du IIIe s. En céramique commune grise, qui totalise $41,2 \%$, l'existence d'un seul ovoïde à bord droit (fig. $72 \mathbf{~ n}^{\circ}$ 4), très fréquent dans la deuxième moitié du IIIe s., témoignerait du début de la production de ce type de matériel. L'absence d'africaine $\mathrm{C}$ fournit un terminus ante quem fixé aux alentours des années 220/230. La constitution des remblais d'installation de la "maison aux stucs" peut donc se situer dans le premier quart du IIIe s. 


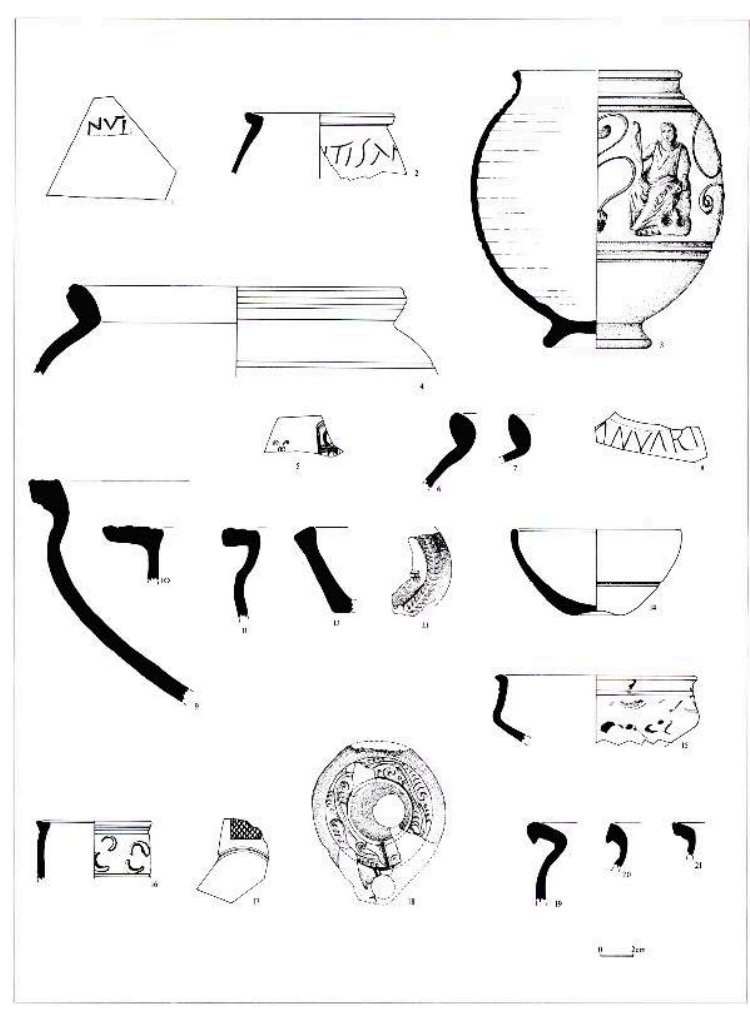

72 - Céramique gallo-romaine : $n^{\circ} 1$ à 12 , période 2 , IIle s., n 13 à 21, période 3 , IVe $\mathrm{s}$.

\section{SOL D'OCCUPATION EN TERRE BATTUE}

Malgré l'échantillonnage très limité de ce lot (20 vases correspondant à 27 tessons), il ressort deux bords de claire $B$ (forme Desbat 67 , très fréquente au début du IIIe s.). A noter un bord de mortier en sigillée gauloise (forme Drag. 45) à décor de cartouche sur le bandeau (fig. $\left.\mathbf{7 2} \mathbf{n}^{\circ}{ }^{5}\right)^{15}$. Ces indices s'accordent à situer la formation de ce sol à une date contemporaine à l'installation des remblais sur lesquels il repose: l'analyse stylistique des enduits peints de style sévérien, constituant le décor intérieur de la maison et retrouvés dans la couche de démolition, a étayé cette proposition chronologique.

\section{DÉMOLITION DE LA MAISON AUX STUCS}

Le lot céramique issu de ces niveaux est cette fois plus important (711 restes de vases pour 808 tessons). Un fragment d'africaine $C$ intervient pour placer la formation de ce gisement après le premier quart du IIIe s.

Toutefois, l'examen des amphores permet d'avancer dans le siècle. En effet, l'apparition du conteneur hispanique Almagro 50 (Keay 1984, p. 169), commercialisé à partir du milieu du IIIe $s$. ou légèrement après, donne l'occasion d'aborder la deuxième moitié de ce siècle. L'absence de céramique luisante interdit de dépasser la fin du IIIe s. La démolition de la maison aux stucs a pu intervenir durant le troisième quart ou à la fin $\mathrm{du}$ IIIe s. Nous pouvons souligner une augmentation sensible des bords droits en céramique commune grise (fig. $\mathbf{7 2} \mathbf{n}^{\circ} \mathbf{6}, 7$ ), typiques de la deuxième moitié du siècle. Notons également un fragment de céramique commune claire portant une marque incisée non identifiée (fig. $72 \mathbf{n}^{\circ} \mathbf{8}$ ). Les fragments de céramique commune sombre 
présentent souvent un aspect micacé de la surface extérieure ou bien sont recouverts d'un engobe orangé. Il s'agit de marmites à marli horizontal, profilé de cannelures sur la face supérieure (fig. $72 \mathbf{n}^{\circ} \mathbf{9}$ à $\mathbf{1 1}$ ), ainsi que d'une série de plats à parois obliques et à lèvre arrondie ou légèrement aplatie (fig. $72 \mathbf{n}^{\circ} \mathbf{1 2}$ ). Les formes fermées de jattes carénées ou urnes à bord dit en amande sont également bien attestées.

\section{LES AMÉNAGEMENTS DU IVe SIÈCLE (PÉRIODE 3)} romaine, repérée tant sur le chantier de l'îlot Tramassac que sur celui de la rue Carriès, a été regroupé et traité simultanément (402 tessons pour 374 restes de vases). L'élément de datation repose sur un fragment de panse en céramique luisante de forme Lamboglia 1/3. Mais la faible quantité de ce type de production a été, dans un premier temps, considérée avec prudence, à cause d'un risque d'infiltration toujours probable. Néanmoins, cette présence pouvait également signifier le tout début de la commercialisation de cette céramique, (Lamboglia 1963, p. 170). Dans ce cas, la voie gallo-romaine est installée à la fin du IIIe s. ou au début du IVe s. La production de luisante amorce donc ici son démarrage et n'atteindra une respectable vitesse de croisière qu'au cours de la phase suivante. L'identification d'un antoninianus, dont l'émission a été fixée dans la seconde moitié du IIIe s., est venue conforter ce créneau chronologique (cf. infra, l'étude numismatique). De l'abondance des amphores gauloises, qui dominent largement les autres types de production d'amphores avec près de $70 \%$, découle la continuité de l'approvisionnement en vin dans le sud de la Gaule, et donc de l'activité vinicole de ces régions, et ce jusqu'au début du IVe s.

\section{NIVEAUX DE FRÉQUENTATION DE LA VOIE}

L'importance de la céramique luisante (42,2\%), à l'intérieur de laquelle la forme Lamboglia $1 / 3$ figure en très bonne place, situe ces niveaux de fréquentation de la voie au IVe s. Un fragment de lampe de forme Hayes I/Atlante VIII (fig. $\left.72 \mathbf{n}^{\circ} \mathbf{1 3}\right)^{16}$ apporte une précision chronologique supplémentaire: ce mode d'éclairage de provenance africaine est en effet diffusé à partir du milieu du IVe s. L'identification de trois monnaies émises au milieu du IVe s. valide la datation avancée par l'analyse céramique (cf. infra, l'étude numismatique). Là aussi, l'absence de céramique estampée grise fournit un terminus ante quem interdisant d'aborder le Ves.

Parmi le mobilier amphorique, il faut souligner la baisse progressive des importations hispaniques $(1,4 \%$, soit plus de $4 \%$ d'écart avec la phase précédente): ce ralentissement des arrivages reçus de cette région de l'Empire est déjà sensible au cours du siècle précédent. Les amphores non identifiées connaissent une notable augmentation $(56,3 \%)$ doublant leur quota par rapport à la phase précédente. Au sein 
des céramiques culinaires en commune sombre et grise, les bords en amande sont toujours bien représentés.

\section{DES NIVEAUX D'OCCUPATION}

33 Fragments de murs, fosses, comblements et traces de démolition évoquent une occupation mal définie. Seul le rapport chronologique fourni par l'étude du matériel céramique (570 vases pour 611 tessons) a permis d'attribuer ces vestiges à la période 3 . La forte proportion de céramique luisante $(55,4 \%)$ situe ces niveaux dans le courant du IVe s. La forme Lamboglia $1 / 3$ est largement attestée dans ses variantes A (possédant une cannelure au dessus de la carène) et $B$ (sans cannelure et à la carène vive), certains exemplaires portant des traces de peinture blanche sur la partie supérieure du vase. Cette forme et dans une moindre mesure la $2 / 37$ à décor de lunules sur la paroi semblent typiques du IVe s. Le mortier à décor de mufles ou de motifs croisés (Drag. 45), la patère à décor de poinçons triangulaires sur la paroi extérieure (Lamboglia 10) figurent également en bonne place. Des fragments d'assiette à fond orné de spirales de guillochis à la molette et de petites coupes à fond déprimé dont la paroi est divisée par un sillon profond apparentées à la forme Lamboglia 33 (fig. $72 \mathbf{n}^{\circ}$ 14), de petites urnes à épaule carénée et col oblique apparentées à la forme 19 de Lamboglia (fig. $72 \mathbf{n}^{\circ}$ 15) et de petits bols à décors de lunules (fig. $72 \mathbf{n}^{\circ}$ 16) constituent les objets les plus fréquemment répertoriés.

Il ressort de ce lot un fragment de fond de plat en claire $\mathrm{D}$, portant un décor de carré quadrillé en diagonales (fig. $\mathbf{7 2} \mathbf{n}^{\circ} \mathbf{1 7}$ ), et appartenant au style A iii de Hayes attesté à partir de la deuxième moitié du IVe s. (Hayes 1977, p. 219 et 241, n 69, fig. 42). C'est un élément opportun pour proposer une datation plus précise : le troisième quart du IVe $s$. L'absence dans ces lots considérés de céramique estampée grise interdit d'approcher la fin du IVe s.

Une augmentation de la production des mortiers, dont la fréquence avait sensiblement diminué à la fin du IIIe s. et au début du siècle suivant, peut traduire une modification des habitudes alimentaires. L'apparition d'un nouveau modèle de lampe à huile en céramique commune à pâte beige sans engobe et dont la morphologie n'est pas sans évoquer les prototypes africains (la lampe à canal au bandeau décoré) mérite d'être signalée (fig. $72 \mathbf{n}^{\circ}$ 18). En céramique commune sombre, outre les bords en amande toujours bien représentés, il faut noter une variété de bord déversé vers l'extérieur et à l'extrémité pendante (fig. $72 \mathrm{n}^{\circ} 19$ à 21).

\section{LES AMÉNAGEMENTS DU Ve SIÈCLE (PÉRIODE 4)}

\section{LES REMBLAIS D'INSTALLATION DE LA VOIE 272}

Un fragment d'estampée grise à marli décoré de poinçons circulaires (fig. $73 \mathbf{n}^{\circ} \mathbf{2 2}$ ), rapproché de la forme Rigoir 1, place ces rehaussements du niveau de la voie au Ve s. Le caractère minoritaire de ce type de production et le fragment de lampe africaine de forme Hayes I / Atlante VIII A/B (fig. $73 \mathbf{n}^{\circ}$ 23) établiraient le rehaussement de la voie au début du Ve $\mathrm{s}$. La céramique luisante occupe toujours une place prépondérante au sein de la vaisselle fine $(69,2 \%)$, ce qui établit la continuité de sa production durant le début du Ves. 


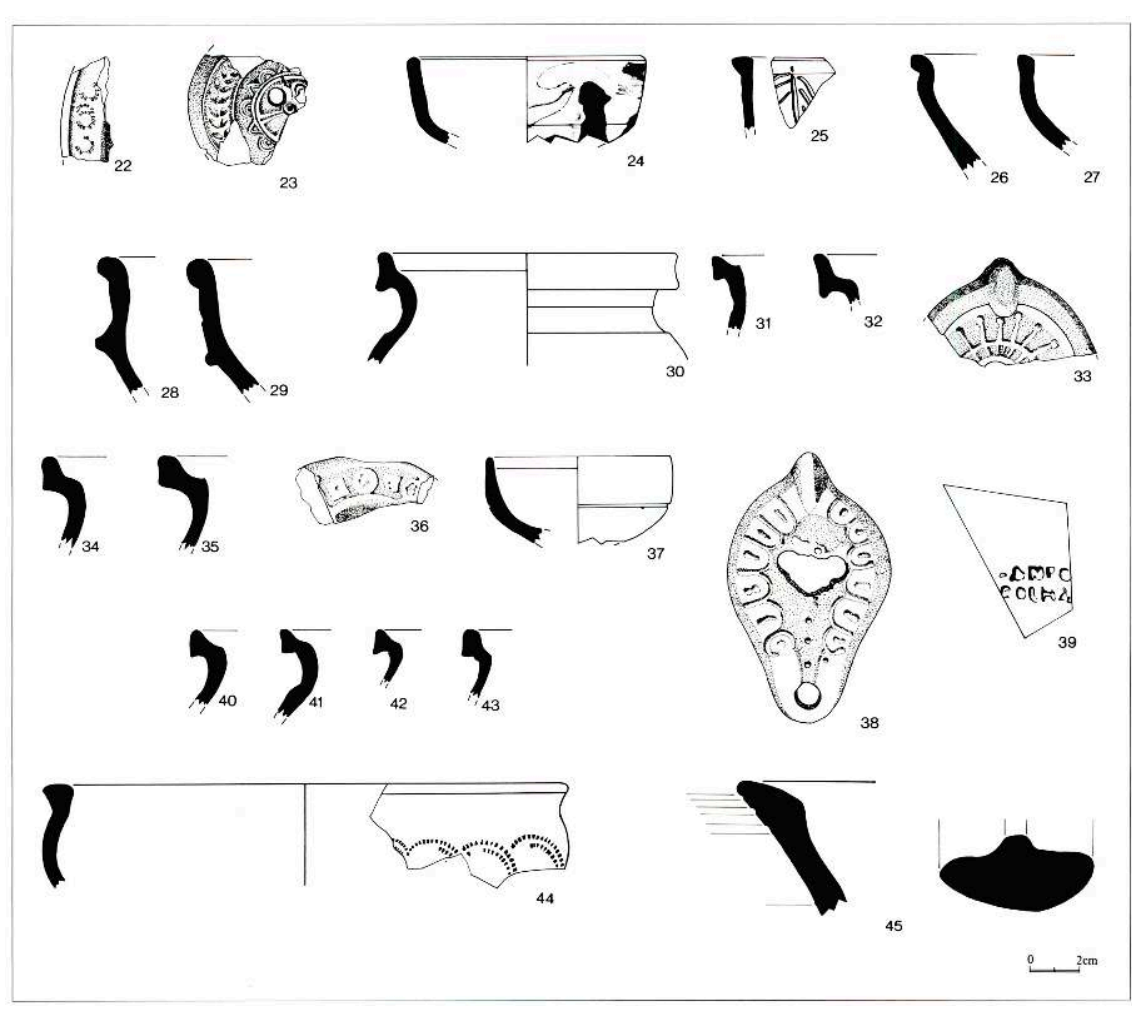

73 - Céramique gallo-romaine : n 22 à 37, période 4, Ve s., n 38 à 44, période 6, Vle s., n 45, bord et anse d'amphore non identifié.

L'augmentation importante des productions d'amphores non identifiées s'impose avec $64,7 \%$.

\section{LE DÉPOTOIR D'OS}

La céramique issue des couches du dépotoir d'os est particulièrement abondante (2150 restes de vases pour 2191 tessons). L'inventaire a révélé une forte proportion de luisante solidement arrimée dans la catégorie des céramiques fines $(53,8 \%)$ : fréquents Lamboglia $1 / 3,2 / 37$, Drag. 45 , mais également coupes à marli Lamboglia $4 / 36$, bols carénés Lamboglia 3 et $3 / 8$, assiettes à bord droit Lamboglia $9 b$, coupelles hémisphériques à bords droits (fig. $73 \mathbf{n}^{\circ}$ 24) ou portant un décor incisé stylisé (fig. 73 $\mathbf{n}^{\circ}$ 25). L'augmentation du pourcentage d'estampée grise (près de $14 \%$ ) est un argument pour un Ve s. bien avancé : bols Rigoir $6 \mathrm{C}$ et 16 présentant un décor de guillochis, coupelles à bord rentrant Rigoir 5 et 34 , ainsi que des assiettes à marli décoré de guillochis, d'incisions rectangulaires ou de motifs géométriques d'arceaux alternés avec des poinçons. Ce type de production, à pâte grise, fine et homogène, revêtue d'un vernis foncé, noirâtre, d'aspect brillant, mais souvent écaillé, a toutefois livré un rare témoin de la variante orangée de cette production : bord à marli de forme Rigoir 1 cependant trop élimé pour en proposer une illustration. Certains fragments présentent parfois une texture si peu soignée qu'il est difficile de les distinguer de la production en commune grise fine.

Parmi les conteneurs d'origine africaine $(9,2 \%)$ ressort un fond très effilé que nous avons rapproché de l'amphore spateion (Panella 1972, p. 105 et 106, fig. 76). Les produits orientaux renferment essentiellement des fragments d'amphore à pâte fine très micacée. Ce conteneur connaît une évolution longue tant du fond que du col, d'abord 
monoansé (Robinson M 282) puis à double anse (Riley L.R.A. 3). Le seul examen des parois rend cependant difficile l'attribution à l'une ou à l'autre des versions. C'est le contexte stratigraphique qui autorise ici le regroupement des fragments à la forme tardive. L'existence d'un fragment de plat Hayes $82 \mathrm{~b}$, portant un décor à la roulette sur la paroi extérieure, semble fréquent dans la deuxième moitié du Ve s. (Hayes 1977, p. 128 à 131, pl. 23).

$40 \mathrm{Au}$ sein de la production en céramique commune sombre, à l'intérieur de laquelle lèvres en amande, coupelles à bords obliques (fig. 73 n $^{\circ} 26$ et 27) et bols à col séparé de la panse par un bourrelet sont encore bien fréquents (fig. $73 \mathrm{n}^{\circ} 28$ et 29), apparaissent des lèvres à bandeau qui peuvent être considérées comme les prototypes des formes en commune grise typiques du Haut Moyen Age (fig. $73 \mathbf{n}^{\circ} \mathbf{3 0}$ à 32). Les contextes issus de la fouille de Saint Pierre-de-Vienne, sous la direction de M. Janet-Vallat, ont également décelé ces formes dans des niveaux chronologiquement identiques ${ }^{17}$.

Durant cette période, apparaît une production à pâte grise, certainement régionale, de lampes à huile au disque orné de motifs géométriques et dont aucun parallèle publié n'a été repéré pour le moment (fig. $73 \mathbf{n}^{\circ}$ 33).

\section{REMBLAI DU SOL 707}

Ce remblai de préparation du sol 707 offre un mobilier dont la constitution, mais non la quantité, est à rapprocher de celui du dépotoir d'os (196 tessons pour 189 vases) : estampée grise de forme Rigoir 1 à marli décoré, ainsi qu'un bol à collerette en luisante, apparentée au $n^{\circ} 24 / 25$ de la typologie de Lamboglia, puis des lèvres en bandeau en commune sombre (fig. $73 \mathbf{n}^{\circ} \mathbf{3 4}$ - 35). Ce niveau installé sur le dépotoir d'os appartient de ce fait à une phase immédiatement postérieure à celui-ci : la fin du Ve s. ou le début du siècle suivant peuvent être avancés.

\section{INSTALLATION DES STRUCTURES PALATIALES}

43 Ces niveaux dégagés sur le chantier de la rue Carriès ont été interprétés comme les vestiges d'une construction monumentale. Si la présence de céramique estampée grise (coupelle Rigoir 34 à paroi ornée de guillochis et fond d'assiette Rigoir 1 orné de motifs géométriques) place ces niveaux dans le courant du Ve s., le fragment de bec de lampe à huile de production africaine identifié à la forme Hayes II / Atlante X (fig. $73 \mathbf{n}^{\circ}$ 36), apporte une précision chronologique supplémentaire en permettant d'aborder la deuxième moitié $\mathrm{du}$ Ve $\mathrm{s}$.

La luisante est toujours majoritaire jusqu'à la fin du siècle avec près de $50 \%$. A noter une forme basse, bol hémisphérique lisse dont la panse est séparée en deux par un sillon (fig. $73 \mathbf{n}^{\circ} \mathbf{3 7}$ ).

\section{LES AMÉNAGEMENTS DU VIe SIÈCLE (PÉRIODES 5 ET 6)}

Les remaniements que connaît l'îlot Tramassac durant ces deux périodes ont eu pour conséquence de perturber la composition du mobilier des niveaux correspondants par un apport de céramiques résiduelles. Devant la pauvreté des informations, la datation est suggérée par les lèvres en bandeau en commune sombre et l'augmentation sensible de la céramique commune grise, deux arguments de l'approche du Haut Moyen Age, donc du VIIe s. 
HABITAT

46 remblais scellant le dépotoir d'os. La position stratigraphique de ces niveaux permet de situer l'installation de ces structures dans la première moitié du VIe s., voire le milieu de ce siècle. A l'intérieur de la céramique fine, la luisante reste non seulement majoritaire par rapport aux autres catégories de céramique, mais augmente de façon marquante $(71,4 \%)$. Les productions non identifiées d'amphores se maintiennent toujours à un pourcentage élevé ( $65 \%)$. Il est nécessaire de mentionner l'importance toute relative du matériel livré par la fouille de cet habitat (234 vases représentant autant de tessons).

\section{TROISIÈME NIVEAU DE VOIE 231}

Dans la partie ouest du chantier, un apport volontaire de remblais a surélevé le niveau de circulation de la voie. L'analyse ostéologique a démontré que les os trouvés dans ce remblai sont du même type que ceux du dépotoir de boucherie et qu'ils pouvaient donc provenir de celui-ci. Il pourrait en être de même pour le mobilier céramique qui ne présente pas de caractéristiques particulières. Les réaménagements du niveau de la voie ne peuvent donc être situés que par datation relative. La céramique luisante pulvérise ses records avec $80 \%$ et des productions non identifiées d'amphores affichent $78 \%$. La céramique commune à pâte grise $(54,3 \%)$ distance à présent la céramique à pâte sombre (35,9\%).

\section{DÉMOLITION - INCENDIE}

Parmi la céramique fine, il faut souligner la persistance, mais en plus faible proportion $(5,7 \%)$, de l'estampée grise parmi laquelle se distingue un bol à marli décoré de guillochis de forme Rigoir 3. La céramique luisante représente toujours un fort pourcentage $(55,2 \%)$. Les deux lampes à huile en commune grise (cuisson réductrice) et une forme presque intacte en commune sombre (cuisson oxydante) (fig. $73 \mathbf{n}^{\circ} \mathbf{3 8}$ ) ont une morphologie rappelant les lampes africaines. A noter un fragment d'amphore africaine portant une inscription en creux qui n'a pu être identifiée (fig. $73 \mathbf{n}^{\circ}$ 39). Le pourcentage élevé de céramique commune grise $(48,3 \%)$ et, à l'intérieur de ce type de production, les nombreuses lèvres en bandeau (fig. 73 n $^{\circ} 40$ à 43), s'accordent pour placer cette période 6 à la fin du VIe s., voire au début du siècle suivant.

\section{CONCLUSION}

Sans entrer dans la minutie statistique, ni tirer des conclusions historiques de graphiques qui ne sont, somme toute, que révélateurs des quelques mètres carrés fouillés, la confrontation des données quantitatives, extraites des tableaux chiffrés et traduites sous forme de diagrammes des trois catégories de céramique (fines, communes, amphores), donne matière à formuler quelques observations relatives au volume et à la dispersion de certains types de production, observations forcement schématiques et lacunaires, donc provisoires. Les pourcentages des différents types de production choisis ne suivent pas toujours une évolution linéaire : les couches d'où provient la céramique traitée ne se sont pas formées de façon identique et la part du 
matériel appartenant à chacune de ces productions est donc variable. Ceci explique l'apparence très heurtée des coupes graphiques.

\section{Les céramiques fines}

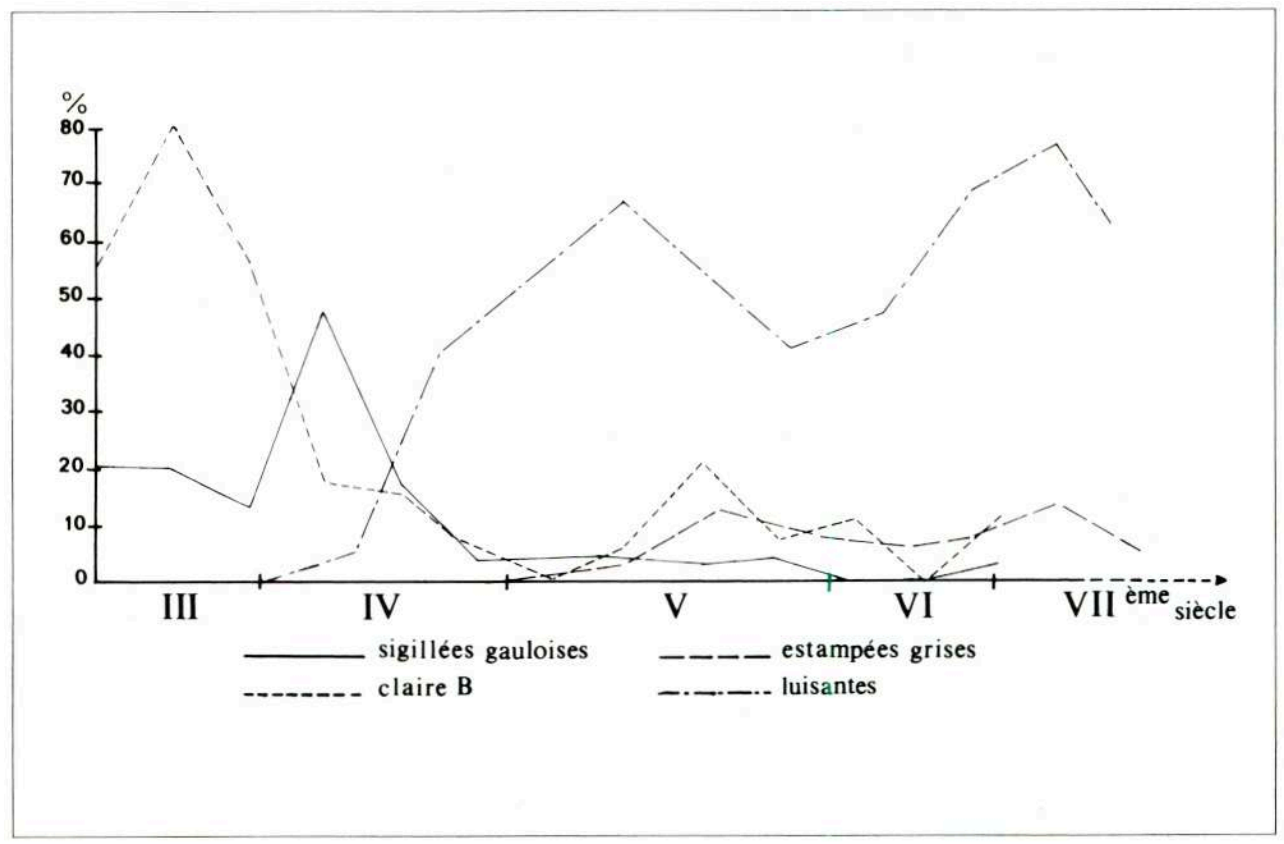

74 - Céramiques fines les périodes les plus récentes. Le problème des fins de production d'une céramique est toujours délicat à cerner : la reprise d'une activité n'est pas exclue, et peut témoigner d'un second souffle des ateliers producteurs, face à des données économiques régionales qu'il est difficile d'expliquer en l'état actuel des recherches sur ce thème. Le tableau quantitatif des céramiques fines obtenues après analyse du matériel du chantier de l'avenue Adolphe Max offre un fort pourcentage de claire B-luisante au Ve et VIe s. : respectivement $89 \%$ et $90 \%$ de l'ensemble de la catégorie (Villedieu 1985, p. 46). Toutefois, le secteur fouillé connait, durant le VIe s, de profondes mutations (apport de remblais, démolition, incendie) qui ont pu fausser la composition céramique 
des couches analysées par un apport de tessons résiduels. Une meilleure connaissance de la céramique luisante dans ces périodes tardives de sa production, donnera la possibilité de vérifier si cette augmentation résulte d'une reprise effective des ateliers (hypothèse peu probable) ou si elle est uniquement imputable à des brassages ponctuels. Les parallèles régionaux font défaut; le problème reste posé. La céramique estampée grise suit, durant le Ve s., une courbe, faible mais croissante tout de même, atteignant son sommet au milieu du siècle (Per. 4). Mais cette production reste toujours minoritaire par rapport à la luisante. Les palmettes, rouelles, arceaux et lignes de pointillés sur la céramique estampée grise ont permis de localiser l'origine de ces décors dans le Languedoc oriental (la production de céramique estampée orangée est également attestée dans cette région). Cependant, le repérage en surface d'un atelier d'estampée grise et orangée près de Valence présentant les mêmes caractéristiques décoratives pourrait attester une origine plus proche de ces produits ${ }^{21}$. La reprise sensible de l'estampée grise au VIe s. suit une évolution identique à la luisante durant la même époque, évolution à laquelle on peut apporter les mêmes explications.

La céramique métallescente, relativement abondante dans les niveaux du IIIe s., en régression constante durant le IVe s., connaît dans les niveaux du Ve s. une augmentation. Résurgence de matériel résiduel ou reprise ponctuelle d'ateliers producteurs? La question est posée.

Si les productions de céramiques fines originaires des régions méditerranéennes, à l'intérieur desquelles la claire $\mathrm{C}$ se distingue, restent modestes (elles totalisent seulement $3 \%$ de l'ensemble de la céramique fine présentée), leur présence constitue à plusieurs reprises de précieux fossiles directeurs.

Il importe de remarquer l'absence de fragments de lampe à huile dans les niveaux du IIIe s. En effet, la faveur de ces petits récipients connut, durant cette période, un net fléchissement dont la cause peut être imputable à des difficultés d'approvisionnement en huile qui entraîna certainement un changement des méthodes d'éclairage. L'apparition, durant le IVe s., de nouvelles formes de lampes à huile, qui ne pourront cependant pas rivaliser avec le raz de marée des formes à volutes très en vogue durant le Haut Empire, témoigne de la remise en circulation de ce mode d'illumination sur des modèles qui semblent inspirés d'exemples africains. En effet, la diffusion des lampes importées coïncide avec l'apparition de productions certainement régionales qui restent toutefois originales: une identité entre les lampes africaines et celles en commune grise ou sombre est à exclure (les décors sont toujours plus complexes sur les lampes africaines : celles-ci ont pu servir tout au plus de prototypes). 


\section{Les céramiques communes}

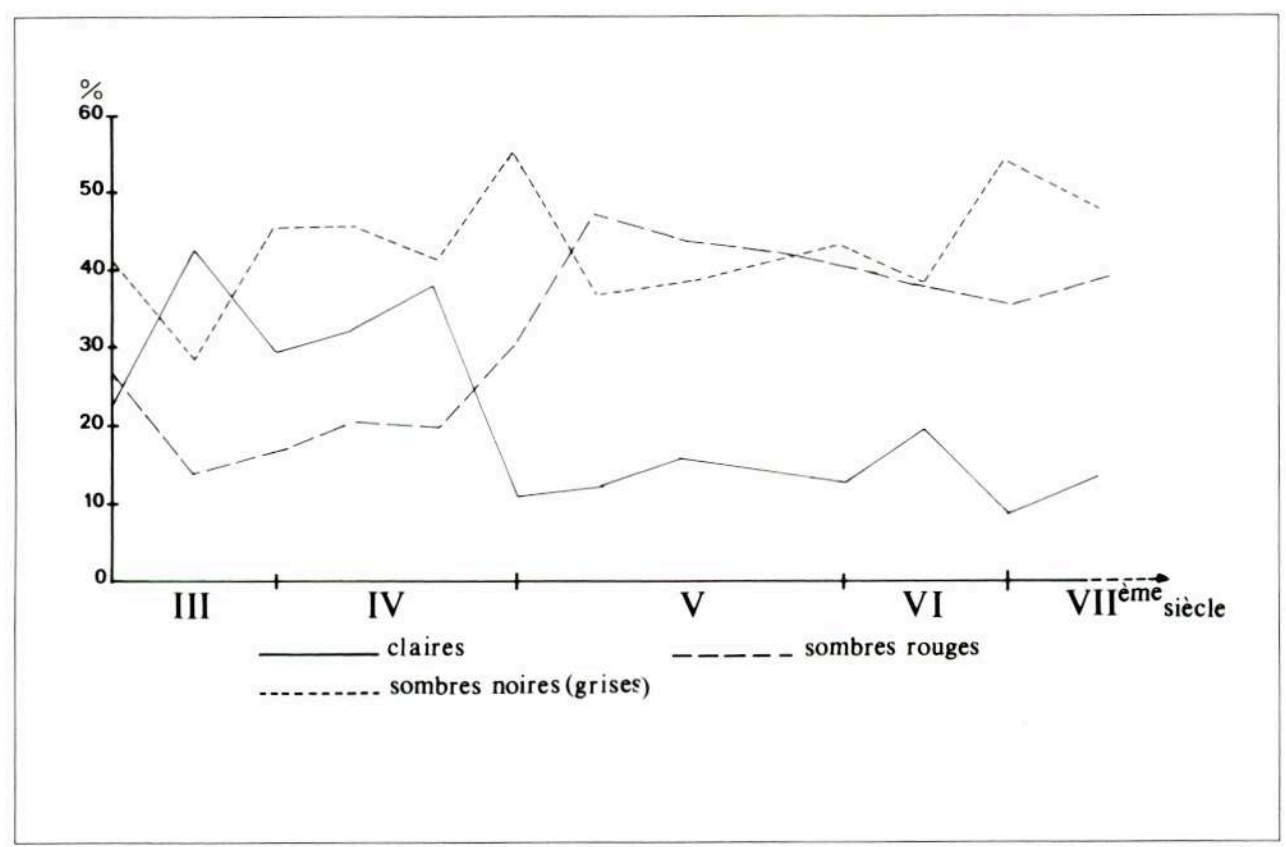

75 - Céramiques communes

58 A l'intérieur des productions utilitaires, la commune claire pâtit d'une chute sensible au IVe s. (37,80 \% en Per. 3-2 à 10,80\% pour la période suivante) et semble relayée par celle de la céramique commune sombre, qui cédera peu à peu du terrain à la commune grise durant le VIe s. (Per. 5 et 6) (fig. 75). L'importance croissante de la commune grise, dont plusieurs exemplaires offrent un décor peigné ou à la molette (fig. $73 \mathbf{n}^{\circ}$ 44), et l'existence, déjà soulignée, des lèvres en bandeau en commune sombre, constituent un indice supplémentaire de l'approche du Haut Moyen Age. Certes, la forme de ces bandeaux en commune sombre, puis grise, connait une évolution qui reste à retracer, mais la filiation semble attestée. Situer une séquence chronologique aussi déterminante que cette époque-charnière est indispensable pour que l'on ne puisse, en connaissance de cause, émettre autre chose que des hypothèses séduisantes, mais chancelantes parce que peu assurées. 


\section{Les amphores}

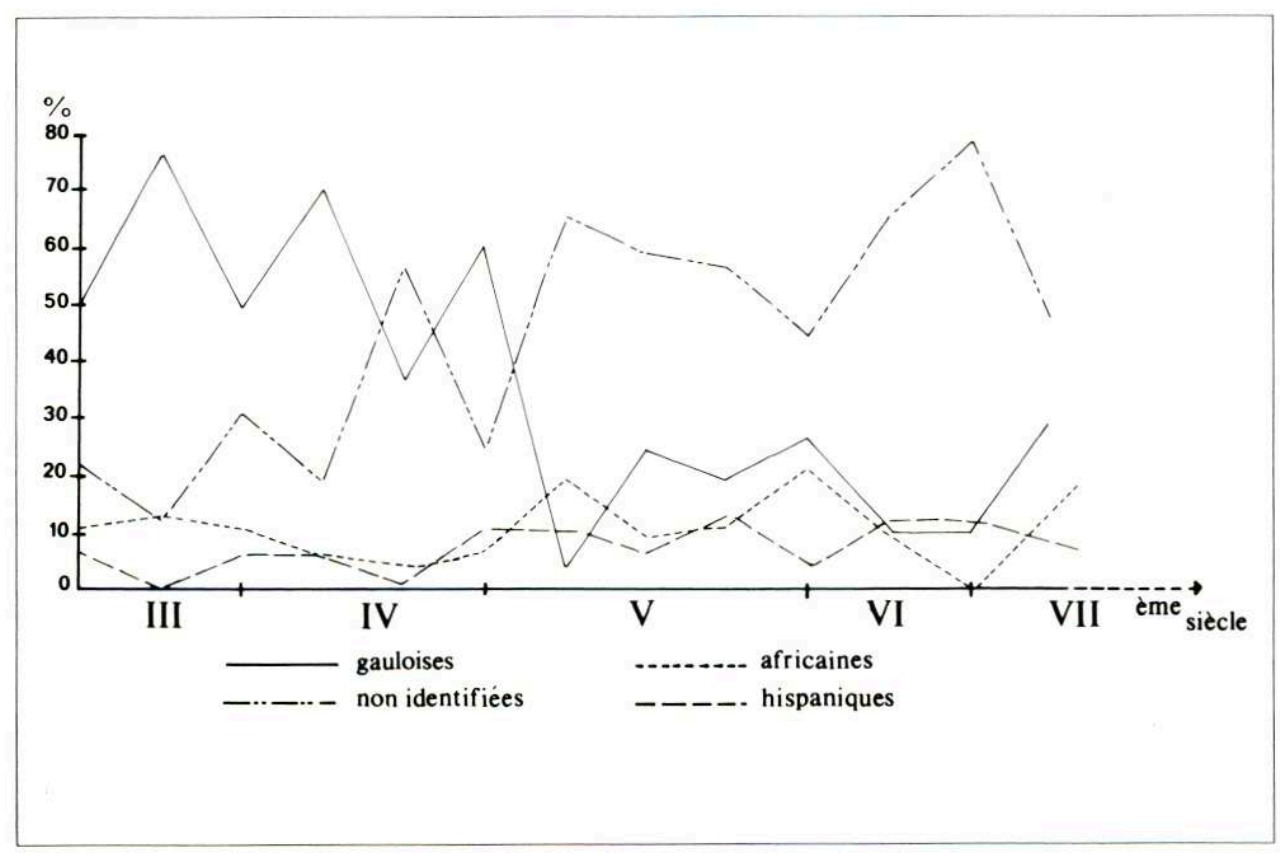

76 - Amphores après le début $\mathrm{du}$ IVe $\mathrm{s}$. Après ce délai, la succession des pourcentages s'avère trop irrégulière pour étayer un commentaire relatif à la production de ce type d'amphore. Cette observation s'applique aux productions gauloises du matériel d'Adolphe Max (Villedieu 1985, p. 46). Les grands ateliers gaulois d'amphores semblent ne pas avoir résisté à la tourmente du IIIe s. Le fort pourcentage des produits non identifiés $(64,70 \%$ en Per. 4) peut être dû en partie au souci d'établir l'attribution d'un fragment à tel ou tel type de production que lorsque celle-ci est parfaitement assurée. Il en découle ainsi une certaine limite dans l'identification. Cependant, des changements dans les aires d'approvisionnement peuvent s'envisager, traduisant une variation des échanges économiques de la région. Ceci impliquerait une diversification des types de production signifiant un éparpillement des centres de fabrication (concurrence grandissante des ateliers domaniaux ?...). Des mutations certaines dans les habitudes alimentaires et/ou technologiques ont dû intervenir. Ainsi, il faut souligner que le volume des productions vinaires de Gaule du sud ( $75 \%$ de l'ensemble des amphores au début du IIIe s.) ne trouvera jamais plus d'équivalent durant toute la fin de l'Antiquité. Désaffection du goût pour le vin (!) ou substitution du tonneau, un important travail reste à faire à ce sujet. Par ailleurs, les importations provenant de la péninsule ibérique (représentées le plus souvent par les amphores à huile Dressel 20 -nous n'avons pas toujours été en mesure de distinguer, au moment de l'inventaire, la forme Dressel 23, avatar de la Dressel 20 - et celles à garum Almagro 50), ainsi que celles des provinces africaines suivent une évolution assez disparate, un type de production semblant prendre le relais de l'autre, mais sans que cela corresponde réellement à des facteurs économiques assurés.

La forme à lèvre biseautée et à anse en ruban large portant un bourrelet médian (pâte assez fine, de couleur beige-marron, recouverte d'un engobe blanchâtre) semble apparaître au cours du IIIe s. (fig. $73 \mathrm{n}^{\circ}$ 45). A noter également une forme fréquente 
parmi les amphores non identifiées, à pâte verdâtre, présentant de nombreuses inclusions vitrifiées et à surface parsemée de petits cratères irréguliers ${ }^{22}$. Parmi les amphores orientales (représentées par les types Kapitan I et II provenant sans aucun doute des régions égéennes et, dans une moindre mesure par la forme "Late Roman Amphorae 4", appelée également amphore de Gaza) se remarque, nous l'avons déjà évoqué, un type bien caractéristique à parois peu épaisses, à pâte fine, bien épurée, de couleur marron foncé, souvent micacée, d'origine probablement égéenne ${ }^{23}$.

61 L'impression que laisse cette étude, somme toute préliminaire et susceptible d'être remise en cause par la poursuite de l'analyse du matériel, ne peut que souligner les limites qui découlent de la méconnaissance de l'ensemble de la céramique de l'antiquité tardive régionale, encore indigente par rapport à celle du midi de la France. Rappelonsle, les éléments de datation proposés, fragiles s'ils sont pris séparément, doivent être considérés avec prudence. Cette fragilité des datations est encore accrue par le système de comparaisons chronologiques basées sur du matériel importé (le décalage déjà souligné entre lieu de production et lieu de consommation). La carence des données comparatives régionales, dont le présent travail fait l'expérience, ne permet pas d'apprécier la validité de la chronologie avancée ${ }^{24}$.

L'examen de ce matériel a donné l'occasion de constater l'abondance et la diversité du matériel céramique réparti dans une succession "serrée" de périodes. Malgré les incertitudes de la destination des structures et des niveaux exhumés, cette abondance et cette diversité constituent un élément certain pour arguer de la réelle continuité de l'occupation humaine dans le secteur Tramassac-Carriès, depuis les premiers établissements à vocation urbaine au début du IIIe s. jusqu'à l'aube du Moyen Age.

Les rapports quantitatifs introduits tout au long de ce commentaire ont permis d'esquisser le grand mouvement de bascule de la fréquence de certains types de production, depuis le IIe s., où la culture matérielle à Lugdunum commence à se détacher des modèles méditerranéens.

Durant cette période, nous avons pu vérifier le maintien des relations de Lugdunum avec les principales régions exportatrices de l'Empire, telles que l'Afrique, l'Espagne et l'Orient. La permanence de ces activités commerciales témoigne de la vitalité du commerce méditerranéen.

$65 \mathrm{Au}$ cours du VIe s., a été rendu sensible le passage d'une nouvelle forme de culture matérielle où est abandonné le vaisselier de tradition galloromaine et la technique de la cuisson oxydante. Cette mutation profonde eut une conséquence socio-économique déterminante pour la région.

\section{G. AYALA}

\section{CÉRAMIQUE MÉDIÉVALE ET MODERNE}

66 Les fouilles effectuées dans l'îlot Tramassac ont restitué 33704 fragments de céramiques médiévales et modernes. L'opération de sauvetage urgent de la rue Carriès, en revanche, n'a livré que peu de matériel pour ces époques. Mise à part la découverte d'une cruche à bec ponté du Xe s., retrouvée dans une fosse, les rares autres tessons, tous en pâte grise, sont restés non identifiables.

67 Cette étude est donc consacrée exclusivement au matériel céramique de l'îlot Tramassac, où l'on a pu suivre, à travers les différentes périodes d'occupation, les 
transformations de la vaisselle domestique en terre depuis l'époque mérovingienne jusqu'à la fin du XIXe s.

Depuis plusieures années, les poteries médiévales et modernes de Lyon ont donné lieu à de nombreuses publications, (Reynaud 1975 ; FaureBoucharlat 1980 a b, 1981 et 1986 ; Becker 1989 ; Maccari-Poisson 1992). De plus, une grande partie du matériel de la fouille de l'îlot Tramassac a été présenté lors de l'exposition "A la fortune du pot" en 1990. Aussi, pour cette publication, nous avons tenu à privilégier les seuls ensembles qui apportent des éléments nouveaux à la typologie connue :

- les céramiques attribuables au Haut Moyen Age,

- les céramiques attribuables aux XIIe - XIVe s.,

- les céramiques de l'extrême fin du XVIe s. et tout début du XVIIe s.

En effet, le développement et l'enrichissement progressif du vaisselier domestique en céramique à Lyon le long de ces dix siècles a été exhaustivement traité lors de l'exposition précédemment citée. Cependant tout n'a pas été présenté; la muséographie retient davantage les formes complètes ou partiellement cassées, or l'archéologie restitue le plus souvent des formes incomplètes. C'est ainsi que la période mérovingienne n'a pas pu être intégrée dans les thèmes principaux de l'exposition et que, de l'ensemble du matériel des XIIIe - XIVe s., n'ont été privilégiées que les formes les plus particulières ou les mieux conservées.

Quant à la collection des vases de la fin du XVIe s., nous avons décidé de la mettre en exergue, car elle couvre une importante période de transition entre deux traditions artisanales : la fin du Moyen Age et le début de l'époque moderne. C'est, en effet, dans le courant de ce siècle que s'imposent les caractères essentiels de toutes les productions postérieures.

\section{LES CÉRAMIQUES DE L'ÉPOQUE MÉROVINGIENNE}

71 Les céramiques attribuables à l'époque mérovingienne ne sont pas nombreuses (environ 300 tessons) et restent très fragmentaires. Néanmoins leur présence est significative pour attester la fréquentation du site à cette époque et pour mettre en place une première typologie des formes et décors attribuables aux VIIe-VIIIe s., encore relativement mal connue à Lyon et dans sa région. Ces céramiques proviennent en partie du sol d'occupation d'un bâtiment dont un foyer a été mis au jour près de l'angle formé par deux fragments de murs liés à la terre. D'autres fragments proviennent, en revanche, de couches de démolition composées de matériaux très hétérogènes.

La chronologie envisagée (VIIe-VIIIe s.) repose sur des comparaisons typologiques établies avec le matériel provenant d'autres sites lyonnais ou de la région proche. Bien que réduit, cet ensemble constitue un groupe très homogène et est composé, exclusivement, de céramiques à cuisson réductrice en pâte grise, généralement assez grossière et comportant de nombreuses inclusions blanches, parfois de gros module.

Parmi les formes identifiables, les cruches à bec pincé sont assez courantes (fig. 77). Le fond est plat et dessine un angle à la liaison avec la panse, qui est globulaire. Le diamètre maximum se situe environ aux $2 / 3$ de la hauteur totale. Le rebord est constitué d'un court bandeau concave sur la face interne. L'anse, opposée au bec pincé, est plate et collée contre le rebord. Le profil de ce vase est très proche de la cruche qui 
a été retrouvée lors des fouilles du cimetière de Saint-Laurent à Lyon et qui a été datée des VIIe - VIIIe s. (Faure-Boucharlat 1986).

Un autre exemple de cruche porte sur le haut de la panse un décor incisé ondé effectué avec un outil pointu (fig. $78 \mathbf{~ n}^{\circ} \mathbf{1}$ et fig. 79). La lèvre, en bandeau, est très courte et l'anse, attachée contre le rebord, a un profil bifide.

Quelques autres fragments de forme fermée sont ornés d'incisions à la molette. Les motifs sont très simples et se limitent à des séries de triangles ou de petites barettes verticales (fig. 80). Les formes, trop fragmentaires, ne permettent pas de déterminer s'il s'agit de cruches ou d'oules. Ce type d'ornement se retrouve sur les deux sortes de pots. Les incisions à la molette sont largement répandues dans la région pour cette époque, même si l'on est loin de l'exubérance et de la variété que l'on rencontre dans le Nord de la France (Colardelle 1983, pl. 244,413 et 418 ; Faure-Boucharlat 1993, fig. 132 et 134).

Aussi nombreux sont les fragments de gobelets, récipients ouverts dont les parois faiblement divergentes forment un tronc de cône renversé. La lèvre, confondue avec la paroi, se referme légèrement vers l'intérieur ; son extrémité est tantôt arrondie, plate ou biseautée (fig.78 $\mathbf{n}^{\circ} \mathbf{2}$ à 5). Les fonds sont toujours plats avec une dépression centrale (fig. $78 \mathrm{n}^{\circ} 6$ et 7 ). Les gobelets sont tous ornés de lignes incisées au peigne, parallèles ou ondées (fig. 81, 82). Cette forme de récipient, ainsi que la cruche à bec pincé, sont bien attestées dans la région pour les VIIe-VIIIe s. Plusieurs fragments semblables proviennent du cimetière de Saint-Laurent à Lyon (Faure-Boucharlat 1987, fig. $9 \mathrm{n}^{\circ} .1$ à 4 et $\mathrm{n}^{\circ}, 6$ ). D'autres gobelets ont aussi été mis au jour dans des contextes du Haut Moyen Age à Seyssel, en Haute Savoie, ou à Argilly en Côte-d'Or (Des Burgondes à Bayard 1981, fig. 179 ; Bourgogne Médiévale 1987, fig. 85).

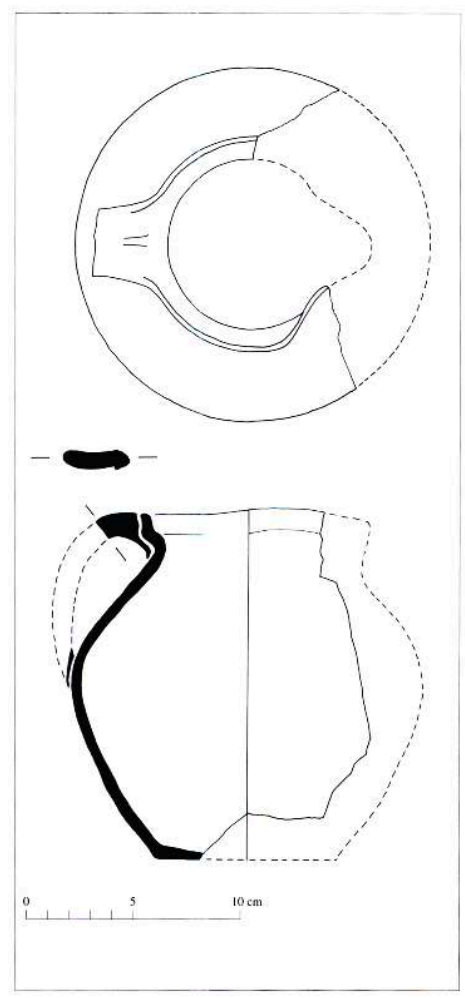

77 - Cruche à bec pincé en céramique grise d'époque mérovingienne 


$$
\because b
$$



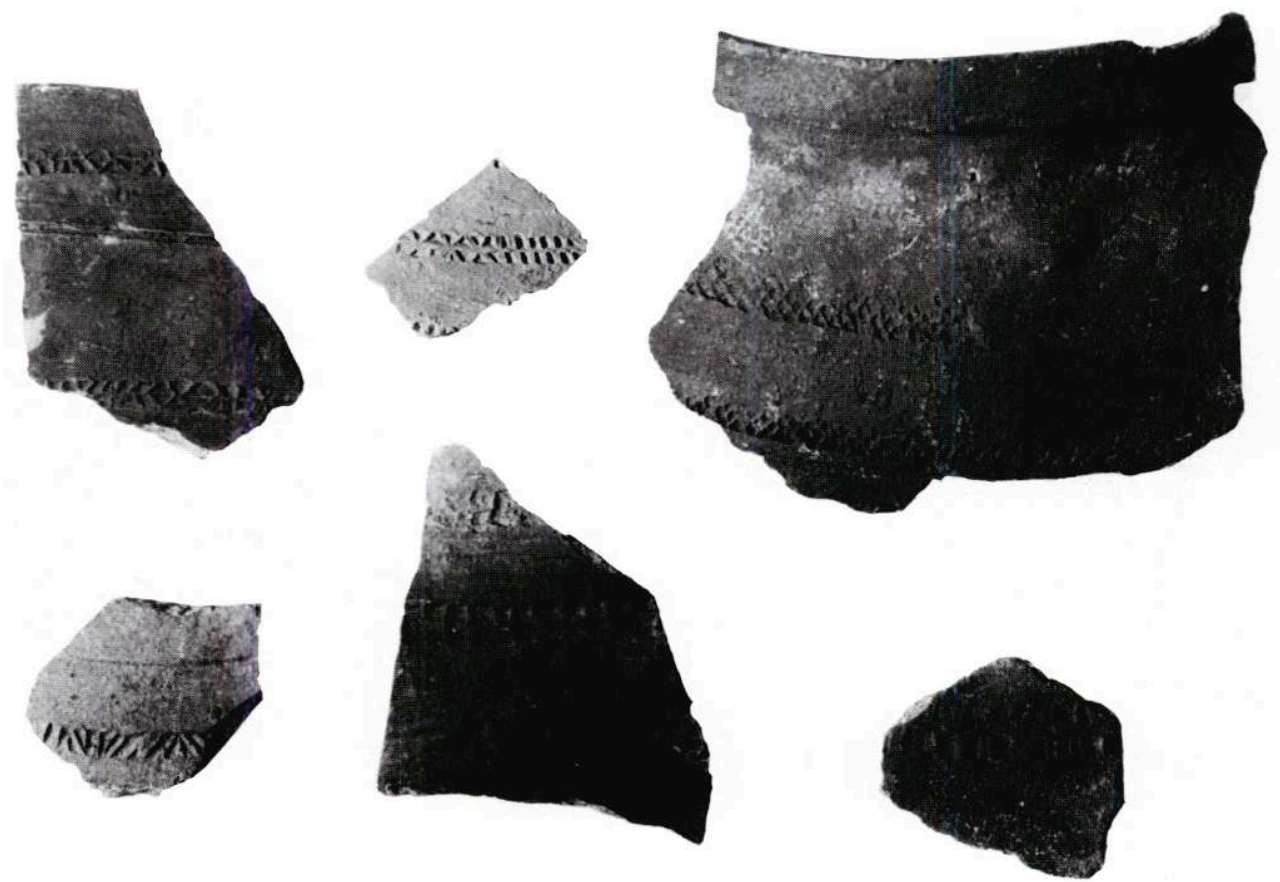

80 - Fragments de formes fermées décorées à la molette d'époque mérovingienne (Cliché P. Plattier)
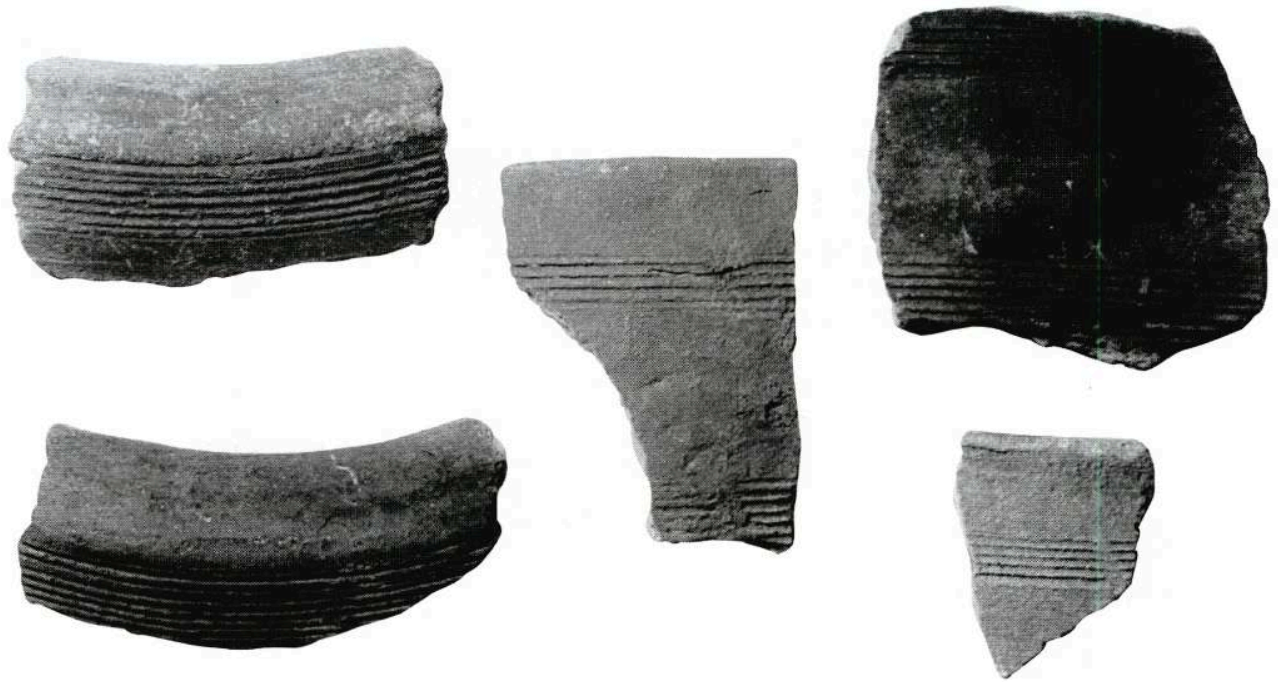

81 - Fragments de gobelets avec décor de lignes parallèles, au peigne, d'époque mérovingienne (Cliché P. Plattier) 

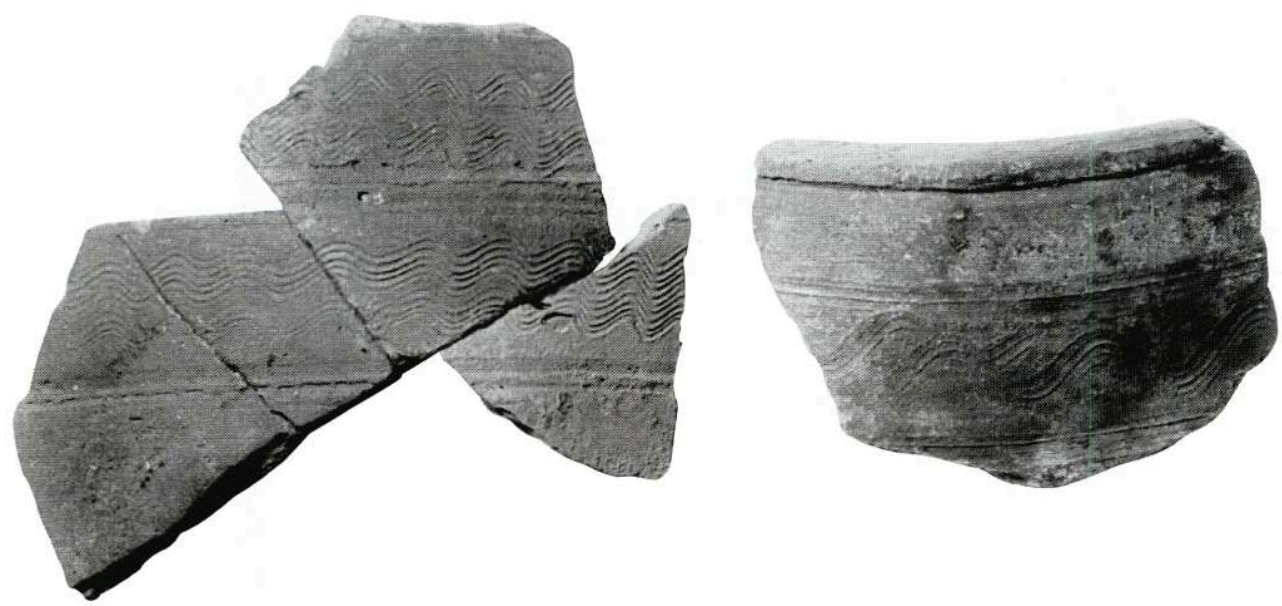

82 - Fragments de gobelets avec décor de lignes ondées, au peigne, d'époque mérovingienne (Cliché P. Plattier)

77 Bien que réduit, cet ensemble de céramiques mérovingiennes complète et confirme la typologie connue dans la région, apportant de nouvelles données, surtout en matière de décor.

\section{LES CÉRAMIQUES DES XIIIe-XIVe SIÈCLES}

Les céramiques de cette époque proviennent en grande majorité du coeur de l'îlot Tramassac à l'emplacement des “jardins" de l'Hôtel du Doyenné, où a été mise au jour une fosse dépotoir. Son remplissage a restitué un mobilier très homogène et les collages effectués prouvent qu'il s'agit d'un comblement qui a été réalisé en une seule fois.

D'autres fragments de vases, plus rares, ont été recueillis dans les couches situées à proximité, mais sont typologiquement comparables au premier groupe. Deux formes proviennent d'une petite fosse de la zone I et une autre a été retrouvée tout au fond du puits 601 avant qu'il ne soit transformé en dépotoir aux XVe et XVIe s. La présence de cette unique forme dans un contexte plus tardif avait déjà été signalée (MaccariPoisson 1988).

La chronologie proposée est fondée sur la comparaison avec d'autres ensembles mieux connus, notamment les poteries mises au jour dans un silo du château des Tours à Anse, dans le Rhône, attribuées au XIIIe s. (Vicard 1984). Néanmoins si les ressemblances avec le matériel d'Anse sont nombreuses, un grand nombre de formes et de décors s'en écarte nettement. Ces différences ne semblent pas pouvoir être attribuées à une époque antérieure au XIIIe s. lorsque glaçures et décors en relief ne sont pas attestés. Il semble, en revanche, que ces vases puissent plutôt être attribués à la fin du XIIIe s. ou au XIVe s. Cette proposition de datation s'appuie d'autre part sur la présence, dans le même ensemble (fosse dépotoir de la zone V), de fragments de poteries tout à fait exceptionnels.

81 Tout d'abord deux tessons d'un albarello peint en noir sous glaçure bleu turquoise importé du Proche-Orient datable du XIVe s. Les importations de céramiques islamiques en France ont fait l'objet d'une enquête et d'une étude qui a intégré cette rare trouvaille lyonnaise (Thiriot 1992). En second lieu la présence d'un fragment de pied annulaire d'une sigillée d'époque gallo-romaine sur lequel avait été gravé dive 
marie avec une pointe assez fine. M.-O. Mandy a examiné ce tesson et attribuerait ce type d'écriture au XIIIe s., à titre d'hypothèse, étant donné le type de support et le moyen utilisé pour écrire.

Les couches qui forment cet ensemble ont livré un lot de 3802 tessons, parmi lesquels a été recueillie une importante quantité de céramiques d'époque gallo-romaine : 921 tessons, soit $24,2 \%$ de la totalité.

Les 2881 fragments de céramiques médiévales se répartissent essentiellement entre quatre types de production (fig. 83).

D'après les pourcentages établis, on constate que les céramiques à cuisson réductrice sont encore très nombreuses, mais parallèlement existe une large diffusion des productions à cuisson oxydante, qui n'apparaissent jamais avant le XIIIe s. Toutefois, la céramique "grise" n'est plus utilisée que pour des vases à usage culinaire. Les formes identifiables ne sont pas nombreuses, par rapport au nombre de fragments recueillis. Ce mobilier est constitué en majorité de tessons de panse extrêmement fragmentés qui n'ont pas permis de remonter la totalité des vases. Les éléments identifiables se réduisent souvent à des rebords ou à des fonds, sans que l'on puisse préciser s'ils appartiennent aux mêmes individus.

\begin{tabular}{|c|c|c|c|}
\hline Type de céramique & Fragments & $\%$ & Formes identifiables \\
\hline Cuisson réductrice & 1862 & 64,7 & 23 formes culinaires \\
\hline Cuisson oxydante sans revêtement & 57 & 1,9 & 1 vase à liquide, 1 tirelire \\
\hline $\begin{array}{l}\text { Cuisson oxydante à revêtement } \\
\text { de glaçure incolore et granuleuse }\end{array}$ & 896 & 31,1 & $\begin{array}{l}38 \text { vases à liquide } \\
4 \text { tirelires }\end{array}$ \\
\hline $\begin{array}{l}\text { Cuisson oxydante et } \\
\text { revêtement de glaçure verte }\end{array}$ & 66 & 2,3 & 3 vases à liquide \\
\hline Total & 2881 & 100 & 70 \\
\hline
\end{tabular}

83 - Répartition des céramiques médiévales attribuables aux XIIle - XIVe s.

Le reste du mobilier est, en revanche, en pâte à cuisson oxydante $(35,3 \%)$ et, sauf rares exceptions, n'a restitué que des vases à liquide. Bien que minoritaire, ce type de production a permis d'identifier au moins 48 vases, car les récipients n'étaient cassés que partiellement.

Les fragments sans revêtement sont rares (1,9\%). Et encore, on ne peut pas exclure que les formes auxquelles ils appartiennent n'aient eu quelques taches de glaçure ailleurs. A cette époque, le revêtement vitreux est encore utilisé avec une extrême parcimonie.

Le type de revêtement le plus courant et le mieux attesté est sans doute la glaçure incolore, saupoudrée sur la paroi externe des vases de façon irrégulière et formant une myriade de petites taches, plus qu'une superficie couvrante et compacte. Selon les formes, la glaçure ainsi parsemée est tantôt le seul ornement attesté, tantôt est associée à un autre décor (bandes de barbotine ou, plus rarement, incisions).

La glaçure couvrante et teintée est peu fréquente (2-3\%) ; dans un seul cas on constate l'utilisation de deux couleurs (fig. 84). Cet objet, de toutes façons, constitue également une exception parce qu'il est aussi l'unique exemple de pot en pâte oxydante de couleur très claire, presque blanche.

Mis à part cet exemplaire isolé, toutes les pâtes à cuisson oxydante sont de couleur rose ou rouge. En grande majorité, elles sont fines, compactes et sans inclusions visibles à l'œil nu (39 formes). Plus rares sont celles à texture plus grossière et comportant des inclusions visibles, généralement blanches (8 formes sur 47 ). Cette diversité de texture 
ne correspond pas, pourtant, à des différences de formes et ne permet pas d'isoler un groupe particulier.

90 Le type de céramique à cuisson oxydante n'est apparemment utilisé que sur des vases à liquide ou sur des tirelires. Pour cette période, il n'existe aucun exemple de forme ouverte pour la table.

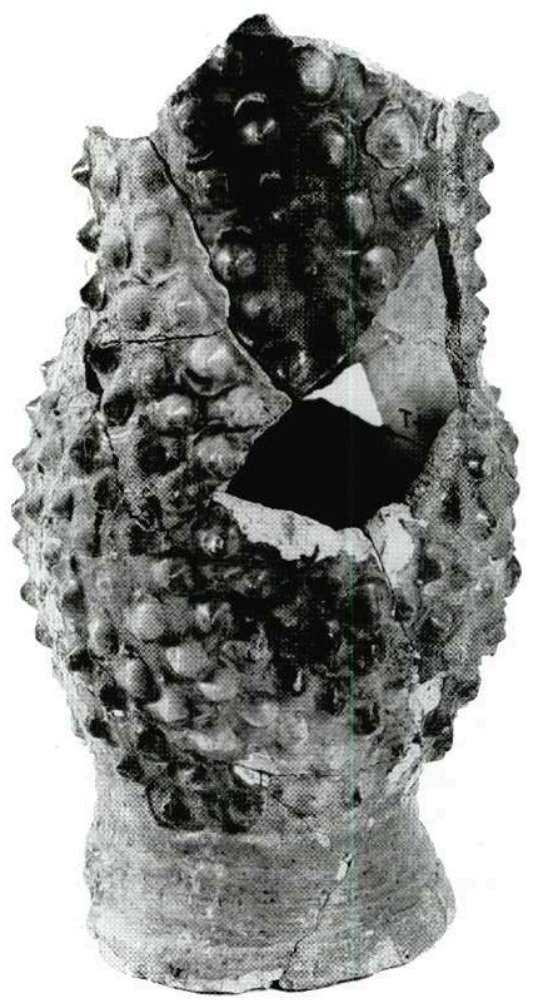

84 - Cruche en pâte claire, décorée d'écailles en relief et recouverte de glaçure bicolore jaune et vert, XIIle - XIVe s. (Cliché P. Plattier) 


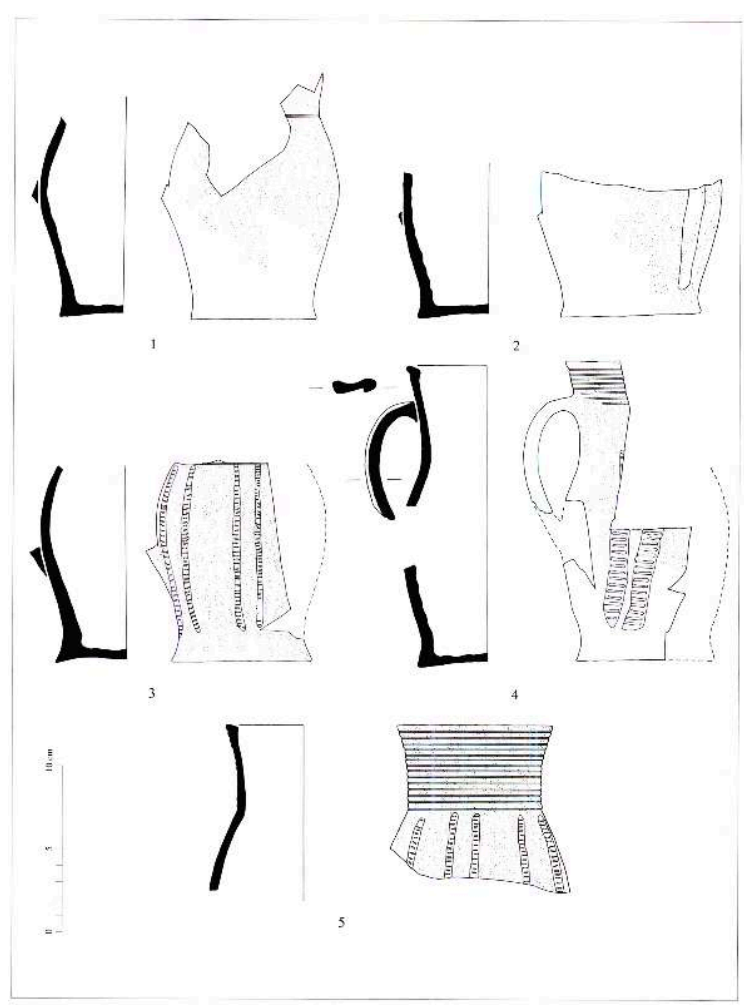

85 - Céramiques de table en pâte rouge et glaçure parsemée, XIIle - XIVe s.

\section{CÉRAMIQUES À CUISSON OXYDANTE ET GLAÇURE PARSEMÉE INCOLORE}

91 Dans cette série, le revêtement vitreux est peu couvrant, d'aspect granuleux et saupoudré irrégulièrement; les formes les plus courantes et les mieux attestées correspondent essentiellement à deux profils : profil ovoïde, et profil en "tonnelet", auxquels s'ajoutent en outre des tirelires et quelques formes rares.

\section{Vases à profil ovoïde}

A quelques détails près, ce groupe s'inscrit dans une grille d'éléments morphologiques identiques : le fond est toujours à base étalée, légèrement concave au milieu, il s'évase à la périphérie, formant un angle aigu très saillant. Le corps du vase est allongé et le diamètre maximum est à peine supérieur à celui du fond. L'anse, plate, a son attache inférieure sur le diamètre maximum ou juste au-dessous. L'ouverture semble être circulaire, en général, mais l'état de conservation des poteries ne permet pas d'exclure la présence, parfois, d'un bec pincé.

Cet ensemble de vases est celui qui se rapproche le plus du mobilier retrouvé à Anse (Vicard 1984) tant par la forme que par l'ornementation. En effet, les vases de Tramassac sont aussi décorés de bandes de barbotine, à l'exception d'une seule forme isolée (fig. $85 \mathbf{n}^{\circ} \mathbf{1}$ ).

Le décor est toujours disposé verticalement sur toute la panse. Le plus souvent les bandes de barbotine sont blanches. Parfois elles sont isolées et sans ornement supplémentaire (fig, $85 \mathbf{n}^{\circ}$ 2). Dans d'autres exemplaires on les retrouve disposées par couples et décorées d'incisions horizontales pratiquées apparemment avec une molette (fig. $85 n^{\circ} 3,4$ et 5). 
D'autres vases sont décorés de bandes de barbotine de couleur brune, qui ne comportent jamais d'incisions mais, comme dans le groupe précédent elles sont soit isolées, soit réunies deux par deux (fig. 86).

\section{Vases à profil "en tonnelet"}

Le deuxième groupe de céramiques, en pâte rose ou rouge et revêtement de glaçure parsemée, présente un profil tout à fait particulier qui évoque un tonnelet en bois. Le fond est très large et le bas de la panse est très aplati, comme dilaté, de sorte que le plus grand diamètre se situe dans la moitié inférieure du récipient. L'anse se trouve généralement juste au-dessous de ce point d'expansion maximum. Le rapprochement que l'on peut faire avec un tonnelet en bois est encore accentué par une série de vases qui ont un décor de côtes en relief rappelant les cercles d'assemblage du tonneau (fig. $87 n^{\circ}$ 1).

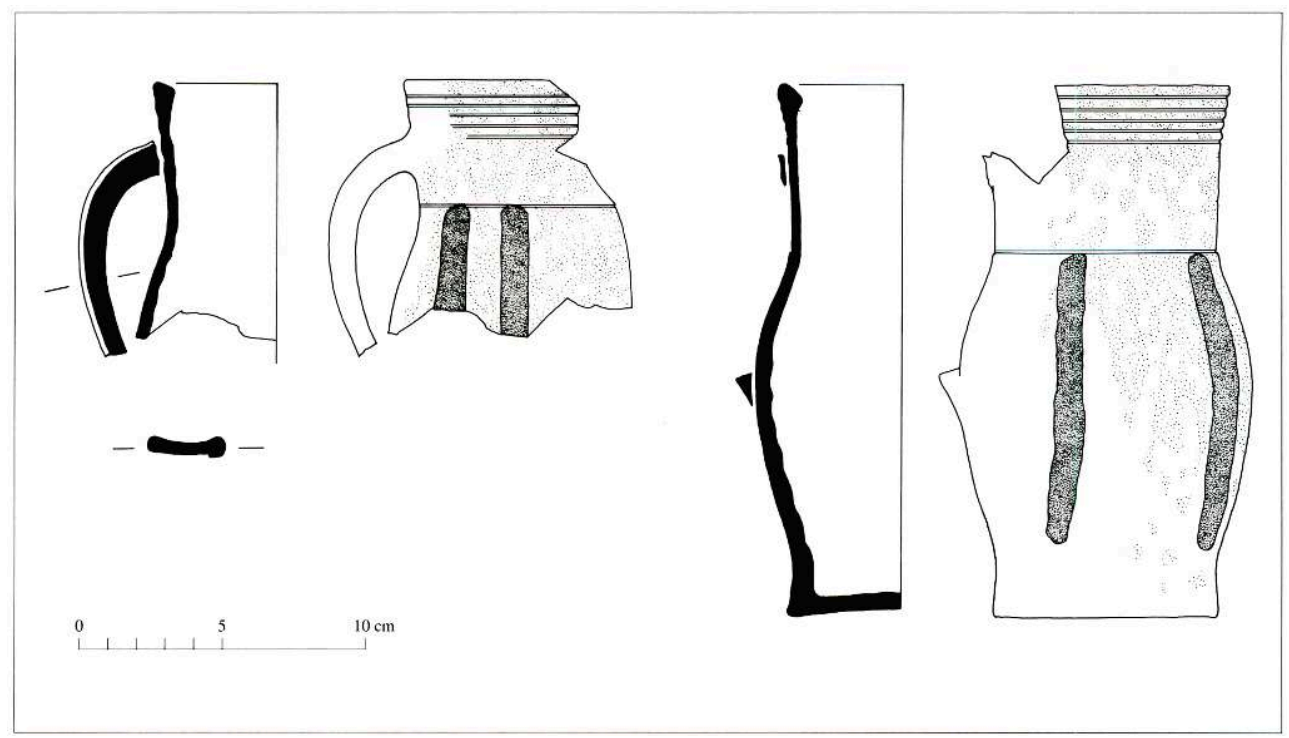

86 - Pichets en pâte rouge et glaçure parsemée décorées de bandes de barbotine sombre, XIIle - XIVe $\mathrm{s}$. 


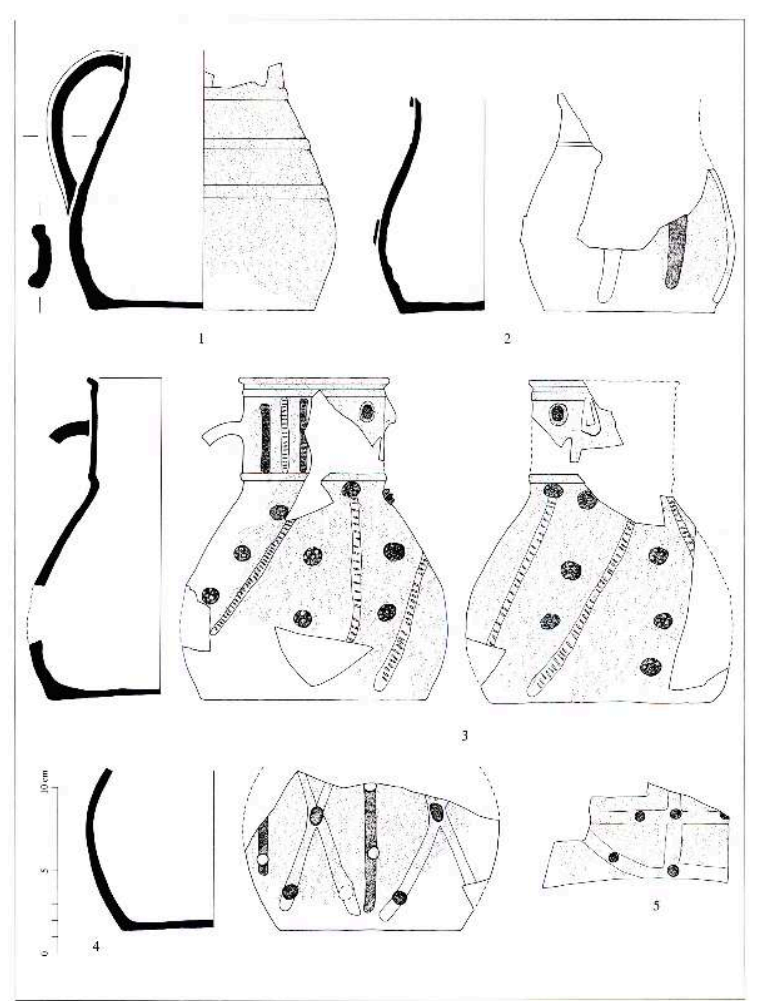

87 - Cruches en forme de "tonnelet" en pâte rouge et glaçure parsemée, XIIIe - XIVe s.

Sur ces vases trapus, les décors, mise à part la glaçure parsemée, sont variés. Outre les côtes en relief, on retrouve encore des bandes de barbotine, mais contrairement au groupe précédent, lorsque les bandes sont disposées verticalement, on constate une alternance de couleurs: blanc et brun (fig. $87 \mathbf{n}^{\circ}$ 2). L'ornementation bicolore se retrouve également sur d'autres vases de ce type, mais le décor est alors disposé en biais formant des lignes croisées (fig. $87 \mathrm{n}^{\circ} 3,4$ et 5).

Les exemplaires les plus singuliers sont les vases qui présentent un décor anthropomorphe (fig. $87 \mathbf{n}^{\circ}$ 3). Un visage humain apparaît sur le devant du col, à l'opposé de l'anse. Sur le côté du visage, le col est décoré de trois bandelettes de barbotine moletées où alternent le blanc et le brun. Le reste du corps de ces cruches est enrichi d'autres bandes moletées disposées en biais et forme ainsi des quartiers où sont appliquées des pastilles en forme de rosette de couleur brune.

Parfois, le décor de bandes de barbotine est très composite: alternance de lignes droites et de lignes croisées en deux couleurs et ponctuées de pastilles en relief de teinte contrastée (fig. $\left.87 \mathbf{n}^{\circ} 4\right)$.

Les décors de barbotine et de pastilles en relief sont largement présents dans le mobilier du château des Tours à Anse (Rhône). Cependant, la forme et les motifs adoptés sur cet ensemble de poteries lyonnaises ne trouvent pas, pour le moment, de comparaison dans la région, à l'exclusion d'une trouvaille ancienne, non datée, appartenant autrefois à la collection du docteur Comarmond (Brongniart 1877).

Quant au décor anthropomorphe, on connaît sa large diffusion dans l'Europe du nord à la fin du Moyen Age. A l'heure actuelle, pourtant, on n'en connait pas d'exemple dans la région, sauf l'exemplaire retrouvé lors des fouilles de la Bastide de Gironville dans l'Ain et qui date du XIVe s. (Poisson 1985). Cependant, les plus récentes fouilles lyonnaises 
apportent d'autres éléments semblables, selon des informations orales de A. Horry, et un décor de ce type a été retrouvé en Franche-Comté, (Jeanjacquot 1993, fig. 75)

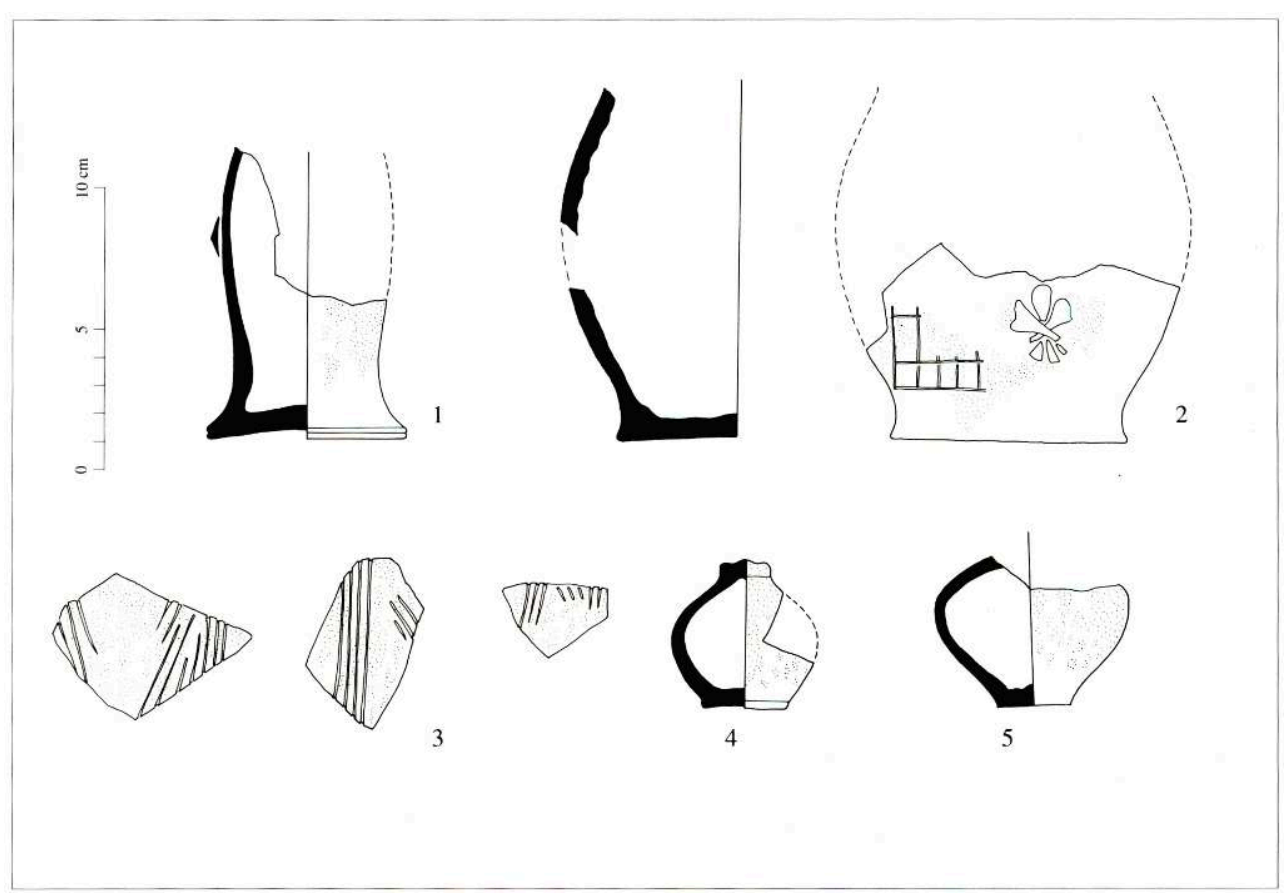

88 - Formes rares en pâte rouge et glaçure parsemée, XIIle - XIVe s.

\section{Formes rares}

A côté des séries répétitives, réunissant les vases à profil ovoïde ou "en tonnelet", les céramiques à glaçure parsemée plus ou moins incolore, réunissent d'autres formes qui restent des exemplaires uniques.

Le premier est un fragment de vase, de très petite taille, dont le corps presque cylindrique est monté sur un fond concave très évasé à la périphérie (fig. $\left.88 \mathbf{n}^{\circ} \mathbf{1}\right)$. Il est en pâte rouge fine, et conserve quelques traces de revêtement vitreux incolore.

Le second est un fragment de pichet orné d'un décor en creux. Le fond de vase, avec une base étalée, est orné sur un côté d'une fleur de lys estampée et entourée de petites taches de glaçure incolore (fig. $88 \mathbf{n}^{\circ}$ 2). Ce décor se trouvait vraisemblablement juste au-dessous de l'anse, puisque sur le côté opposé aucune trace d'élément de préhension n'est visible. Le type de décor estampé est en soi un élément rare mais, en outre, cette forme compte des incisions supplémentaires effectuées après cuisson. S'agit-il d'une marque de propriété apposée par l'utilisateur du récipient, comme cela semble être le cas pour certains vases d'Avignon (Démians d'Archimbaud 1979-80)?

Sur d'autres fragments (peut-être appartenant à un même vase) on retrouve à nouveau un décor incisé mais, dans ce cas, dès l'origine puisque la glaçure a été posée après (fig. $88 \mathrm{n}^{\circ} 3,4$, et 5).

Quatre tirelires, dont la plus petite est conservée presque entièrement, complètent ce groupe de céramiques. 


\section{CÉRAMIQUES À CUISSON OXYDANTE ET À GLAÇURE VERTE}

Les rares fragments de céramique à cuisson oxydante associée à l'utilisation de la glaçure verte n'ont permis de remonter que trois formes, dont deux presque identiques. Le revêtement vitreux teinté a, parfois, un aspect granuleux, mais il est toujours bien couvrant et compact. Sur un vase il est utilisé en guise de décor et non comme revêtement, puisque la tonalité verte sert à orner, avec des bandes obliques, une cruche entièrement recouverte de glaçure plus claire, teintée à peine de jaune (fig. $89 \mathrm{n}^{\circ} \mathbf{1}$ et fig. 84). Cette sorte de spirale verte ne constitue qu'un élément du décor pour ce pichet qui est surtout orné d'écailles en relief du haut du col jusqu'au départ du pied. La forme de ce vase, exceptionnellement en pâte très claire, presque blanche, rappelle de près celle des vases à profil ovoïde et base étalée décorés de bandes de barbotine (fig. 84). Ce pichet orné d'écailles trouve des comparaisons étroites avec le mobilier du XIIIe s. mis au jour dans le silo du château des Tours à Anse (Vicard 1984). Parmi les nombreuses poteries de ce site, a été retrouvé un col de pichet qui pourrait être le complément idéal de la forme lyonnaise qui, elle, ne possède que le corps et le fond.

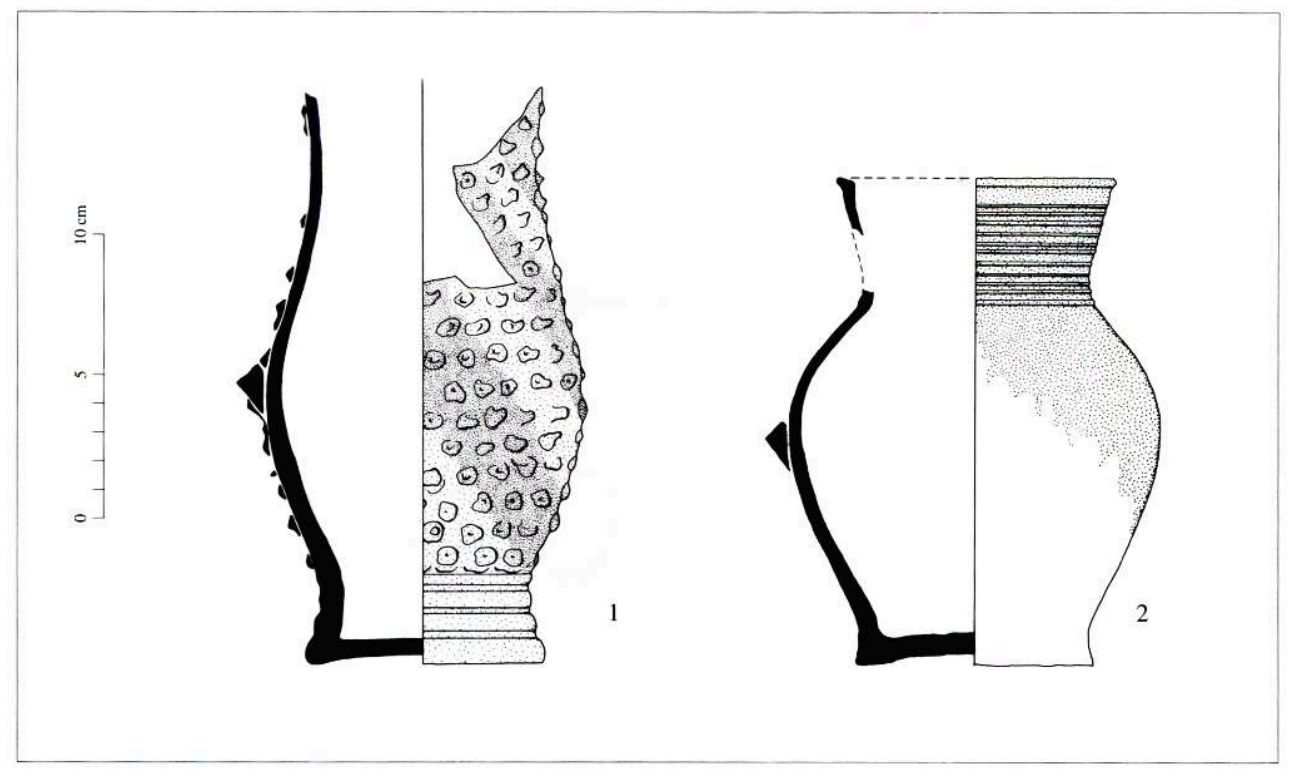

89 - Cruches en pâte rouge et glaçure verte, XIIIe - XIVe s. 


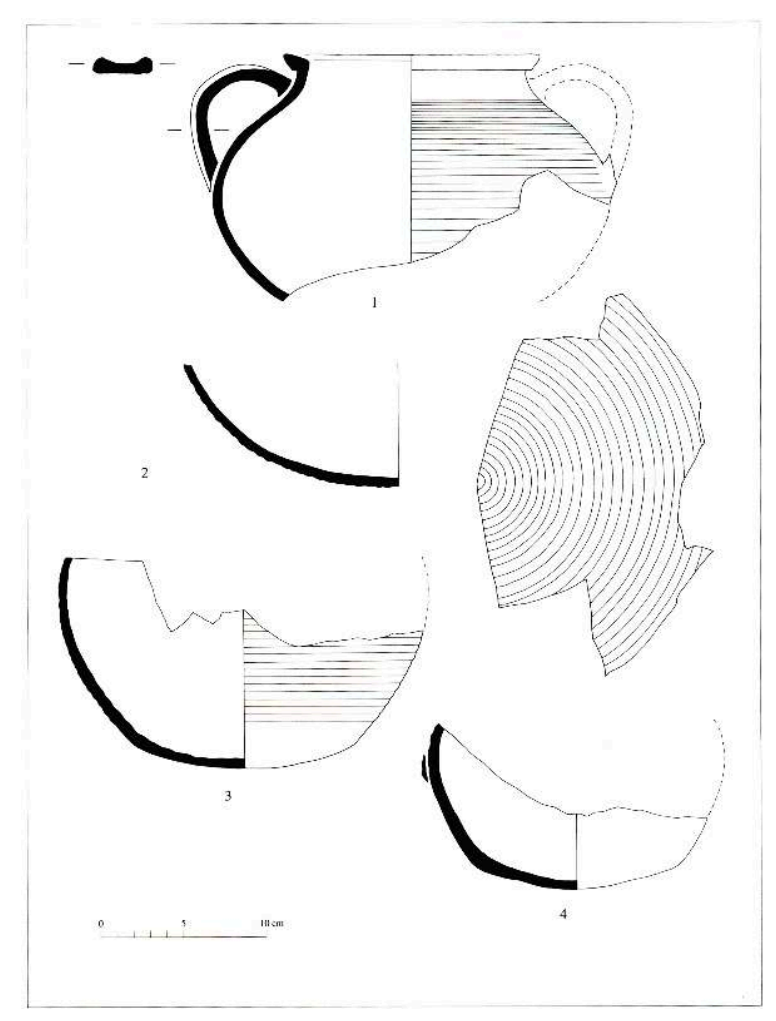

90 - Fragments de marmites en pâte grise, XIIle - XIVe s.

Les deux autres vases, presque identiques, ont également beaucoup d'éléments en commun avec le type 1 des céramiques d'Anse. L'un, cependant, n'est recouvert que partiellement d'une large tache de glaçure du côté opposé à l'anse (fig. $88 \mathbf{n}^{\circ} 2$ ). Le fond, concave, a une base étalée ; le bas de la panse est évasé en tronc de cône renversé et le col, à parois divergentes, est orné d'une série d'incisions. La pâte, rouge, est très fine et compacte sur les deux exemplaires.

\section{CÉRAMIQUES CULINAIRES À CUISSON RÉDUCTRICE}

Les 1862 tessons en pâte grise n'ont permis de restituer que peu de formes. Seuls 23 vases, souvent très fragmentaires, donnent la possibilité d'esquisser une typologie sommaire des pots à usage culinaire contemporains de la vaisselle de table ci-dessus décrite.

Dans les marmites, les pâtes grises sont assez grossières et les parois des vases sont généralement épaisses, sauf à l'endroit où se situe le diamètre maximum (le lieu de fracture le plus courant). En revanche, la pâte est toujours assez fine dans les coquemars, petits récipients munis d'une seule anse.

111 Un premier groupe de vases réunit les marmites dont le départ supérieur des anses se situe au dessous de la lèvre. Le plus souvent ces récipients ont un profil globulaire très aplati et sont entièrement recouverts de cannelures externes (fig. $\left.90 \mathbf{n}^{\circ} \mathbf{1}\right)$. Le rebord, évasé et épaissi, a une section presque rectangulaire. Les deux anses, larges et avec deux bourrelets latéraux, retombent sur le diamètre maximum de la panse.

Par ailleurs un grand nombre de fonds ont été répertoriés. Une catégorie de fonds, dont les stries de tournage se poursuivent jusqu'au milieu, sans qu'apparaisse aucune distinction entre base et paroi n'a pas pu être complétée, mais semble se rapprocher de 
ce type (fig. $90 \mathbf{n}^{\circ}$ 2). D'autres fonds possèdent également des cannelures en relief à l'extérieur (fig. $90 \mathrm{n}^{\circ}$ 3). Ce caractère, pourtant, ne suffit pas à les associer au premier type de marmite identifié précédemment, puisque le profil de la panse semble fort différent. Ces fonds appartiennent à des récipients globulaires qui ne présentent pas la même dilatation du diamètre maximum.

Parmi ces marmites dont les anses sont accrochées au dessous de la lèvre, une variante, plus rare, présente un profil beaucoup plus allongé et presque ovoïde (fig. $91 \mathbf{n}^{\circ} \mathbf{1}$ ). Les cannelures sont, dans ce cas, absentes et à leur place apparaissent deux cordons en relief sous le rebord, comme pour souligner la césure entre la lèvre et la panse sans que l'on puisse parler d'un véritable col.

114 Le second groupe de pots culinaires est formé par des marmites dont l'attache supérieure de l'anse se situe contre la lèvre (fig. $91 \mathbf{n}^{\circ} 2$ ). La panse est encore globulaire, mais le fond tend à une base presque plate, sans pourtant que la liaison fond/panse apparaisse distinctement. La lèvre évasée se confond complètement avec les anses qui sont plates et marquées par une large dépression en leur milieu. On note toujours l'absence d'un véritable col.

115 La troisième série de vases culinaires en pâte grise comprend les coquemars : petits récipients munis d'une seule anse, souvent appelés "pégaus" lorsqu'ils sont mis au jour dans des contextes funéraires (Leenhardt 1988). Ils ont tous un profil globulaire et le rebord évasé, mais se distinguent en deux groupes. Le plus important est celui qui réunit les petits vases avec un fond bombé (fig. $91 \mathbf{n}^{\circ} 3$ et 4). Plus rares sont les coquemars qui présentent un fond légèrement concave et dont la liaison avec la panse est nettement soulignée par un profil anguleux (fig. $91 \mathbf{n}^{\circ}$ ).

Pour la céramique culinaire, comme pour la vaisselle de table, on se trouve donc confronté à deux types de production aux traits morphologiques bien distincts. Devant cette différence on peut bien sûr imaginer qu'ils sont issus d'ateliers différents, mais on ne peut exclure l'hypothèse qu'ils reflètent plutôt l'évolution progressive du vaisselier à la charnière des XIIIe et XIVe s. , époque à laquelle les nouveautés s'implantent parallèlement aux productions de tradition plus ancienne. 


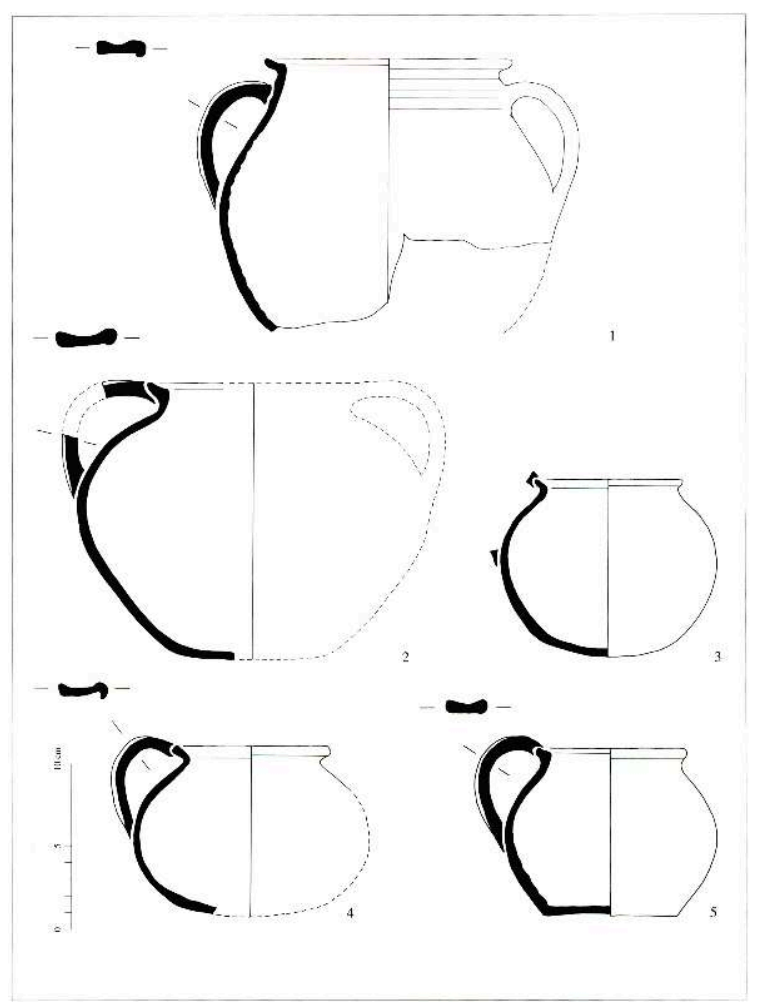

91 - Marmites et coquemars en pâte grise, XIIIe - XIVe s.

\section{LES CERAMIQUES DE LA FIN DU XVIe SIÈCLE}

117 Le mobilier céramique du XVIe s. constitue sans doute l'ensemble le plus remarquable et le plus riche mis au jour dans les fouilles de l'îlot Tramassac. De plus, l'importance de ces poteries est accentuée par les éléments de datation qui leur sont associés et qui permettent d'envisager une chronologie absolue, entre la première moitié du XVIe s. et le tout début du XVIIe s. Deux dépotoirs ont restitué deux ensembles distincts de poteries comparables et homogènes. Le premier ensemble regroupe les céramiques retrouvées dans le comblement le plus tardif du puits 601 (Maccari-Poisson 1988). Dans cette partie du dépotoir a été retrouvée une monnaie royale datée de 1498-1547. La deuxième zone de rejet, (dépotoir IV) a restitué en revanche un jeton de B. de Villars de 1608.

118 On dispose donc de deux jalons chronologiques, la première moitié et la fin du XVIe s. Entre ces deux limites on peut observer la continuité ou la transformation des formes et des types de revêtement et de décor des poteries. Une seule forme se maintient pendant le siècle sans aucune variation importante: celle de la marmite à anses coudées, largement présente dans les deux dépotoirs et dont la production a été constante.

119 En revanche, pour d'autres céramiques on observe de considérables différences entre le début et la fin du siècle. Parmi les autres pots culinaires, les récipients en pâte rouge sont rares dans le dépotoir du puits 601 (Maccari-Poisson 1988). Ils sont par contre très nombreux à la fin du siècle, dans le dépotoir IV. De plus, les coquemars et les marmites de cette époque plus récente sont très souvent glaçurés. Ils semble donc que la 
production des pots culinaires en pâte rouge et glaçure interne se soit généralisée dans le courant du XVIe s.

Dans la vaisselle de table apparaît une distinction encore plus frappante. Les cruches du début du siècle sont encore influencées par les techniques les plus anciennes: taches irrégulières de glaçure éparse, peu couvrante, pratiquement incolore. Le revêtement d'émail ne fait son apparition que sur deux petites formes ouvertes (Maccari-Poisson 1988). Dans le dépotoir de la fin du siècle, en revanche, les faïences ou leurs imitations sont nombreuses, la production de glaçures bien couvrantes, épaisses et avec adjonction de couleurs, est bien représentée.

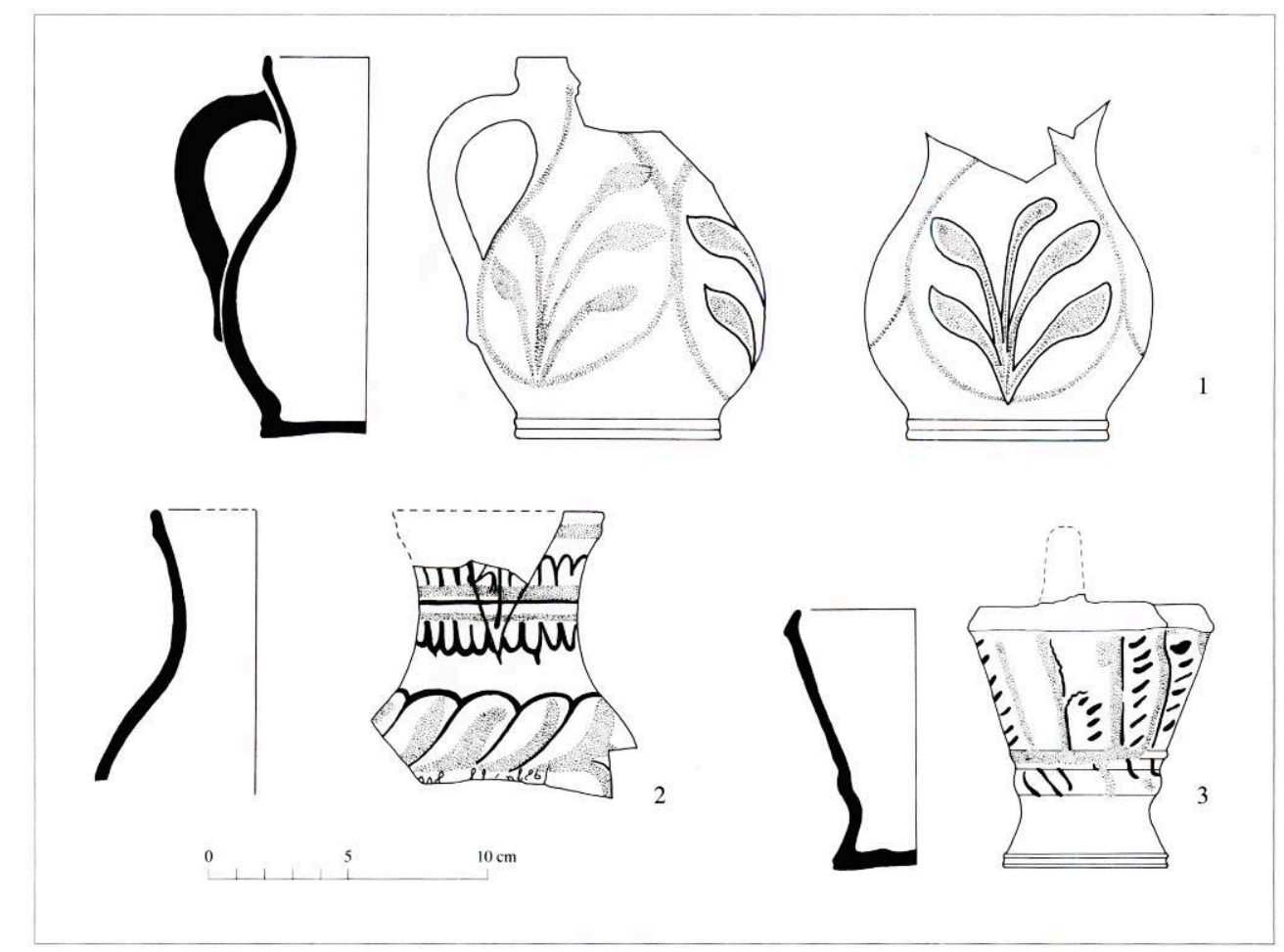

92 - Vases en faïence, début XVIle s.

\section{LA VAISSELLE DE TABLE}

Les poteries de table ne sont pas nombreuses dans le dépotoir IV : sur un total de 86 formes identifiables, elles ne représentent que $22 \%$ de l'ensemble (19 vases). Ces quelques pots offrent cependant un répertoire de formes et de décors assez varié.

\section{Les Faïences décorées en vert et brun}

La première forme, archéologiquement complète, est une petite cruche de profil globulaire (fig. $92 \mathbf{n}^{\circ}$ 1). Son décor, assez altéré, et par endroits à peine visible, est posé sur un fond d'émail blanc qui recouvre la totalité de la surface externe, sauf le fond. Il dessine en vert trois médaillons en forme de lobes pointus qui encadrent des feuillages de couleur verte. Le brun n'apparaît que sur le médaillon central où le rameau feuillu est surligné d'une fine ligne de manganèse.

Bien que très fragmentaire, la deuxième forme permet de découvrir un décor plus articulé : une série de lignes horizontales vertes plus ou moins larges s'alternent, sur le col, avec deux lignes festonnées brunes. Sur la panse, de larges lignes ondées brunes 
encadrent de larges taches vertes. Le vase s'est cassé le long d'une ligne où apparemment était gravée une phrase qui reste, malheureusement illisible (fig. $92 \mathbf{n}^{\circ}$ 2).

Ce groupe comprend aussi un petit vase de forme biconique, muni d'un bec pincé et d'une anse en panier (fig. $92 \mathbf{n}^{\circ}$ 3). Le décor, toujours vert et brun sur fond d'émail blanc, fait alterner des bandes verticales et des séries de petits traits obliques.

Dans ces trois formes de faïence, la pâte, de couleur rose pâle est très fine ; l'intérieur des vases est entièrement recouvert de glaçure transparente. L'émail qui recouvre toute la surface externe, sauf le fond, est de très bonne qualité, bien adhérent, sans craquelures et bien compact.
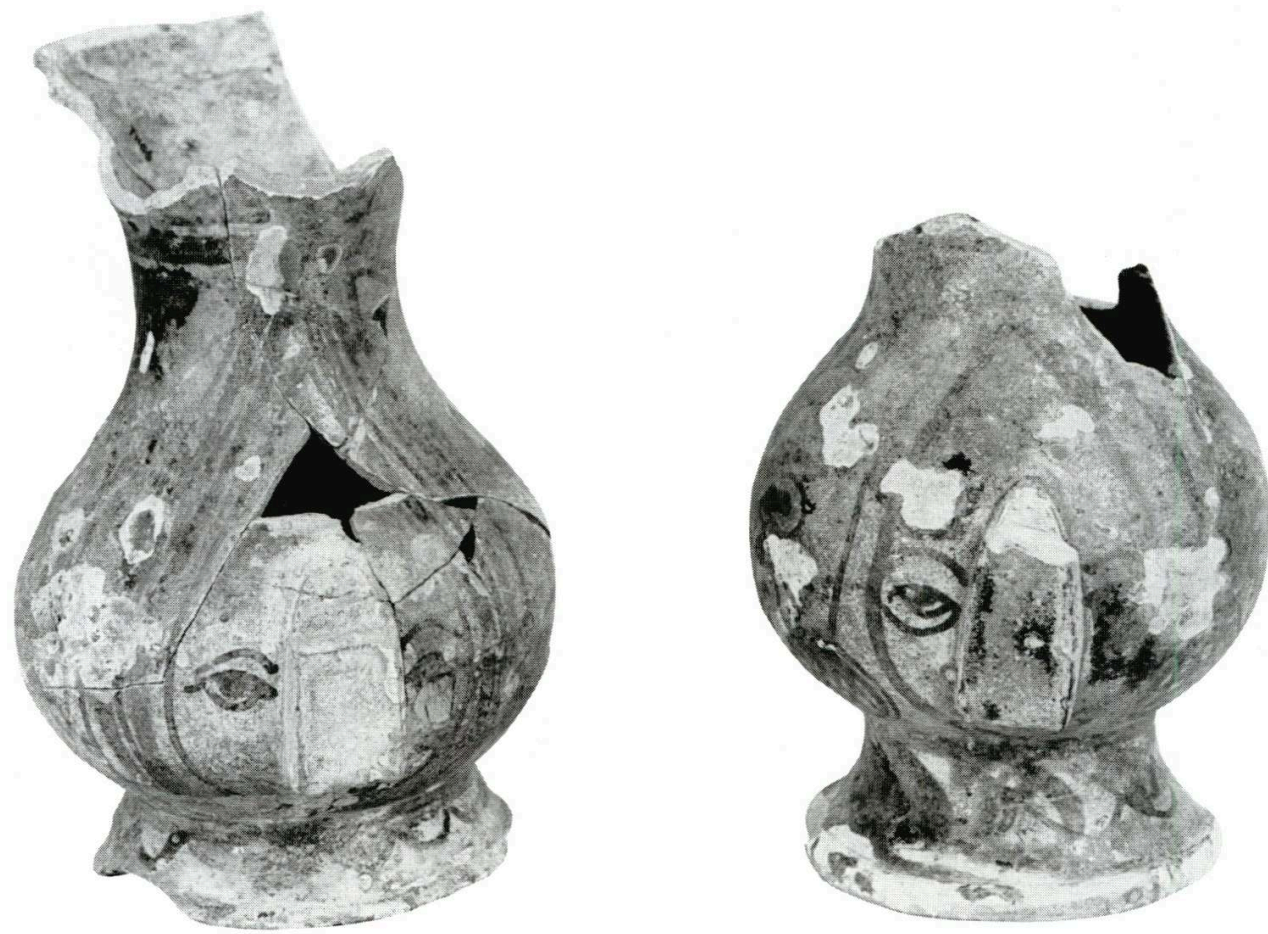

93 - Vases décorés en vert et brun sur engobe blanc, début XVIle s. (Cliché P. Plattier)

\section{Les décors verts et bruns sur engobe blanc}

Deux formes présentent ce type de décor et semblent issues d'un même atelier sinon du même artisan (fig. 93). Leur forme est pratiquement identique : la panse, à profil en bulbe, repose sur un pied largement évasé et se termine en haut par un col très haut. Le décor utilise les mêmes motifs floraux et géométriques. De plus, les vases ont apparemment reçu un revêtement de mauvaise qualité qui s'est fortement écaillé. L'état de dégradation est sans doute dû à un manque d'adhérence entre la pâte et l'engobe, comme si ce dernier n'avait pas suffisamment pénétré le support argileux. Ce mauvais travail de base contraste avec l'habileté du décor qui couvre toute la surface externe. Sur le col et le pied alternent en vert et brun des lignes et des motifs géométriques. Sur la panse, des lignes brunes encadrent des feuillages très articulés et, sur un côté, une figure humaine stylisée. Comme pour le mettre encore plus en évidence, le nez de cette tête est formé d'une excroissance rectangulaire qui sert d'élément de préhension. 
La rareté des trouvailles de ce type de production au XVIe s. dans la région permet de proposer, pour ces deux vases, l'hypothèse d'une imitation des faïences qui font, à l'époque, une forte concurrence aux poteries traditionnelles.

\section{Les décors blancs sur fond d'engobe sombre}

Parmi les poteries de table du dépotoir IV, attribuables à l'extrême fin du XVIe s., ou au début du XVIIe s., apparaissent les premiers témoignages d'une production qui aura, par la suite, une très large diffusion et que l'on appelle couramment "aux engobes" (A la fortune du pot, 1990). Sur un fond d'engobe rouge ou brun, se détache un décor en barbotine blanche recouvert de glaçure transparente. A son apparition, cette production semble se limiter à des formes de petite taille dont il ne reste à Tramassac que quelques fragments (fig. 94): deux fragments de tenons d'écuelle, deux fragments de petites formes fermées, un fragment de petit plat, et enfin un fragment de couvercle.

\section{Les glaçures vertes sur pâte blanche}

C'est à la même époque que l'on retrouve aussi les premiers tessons d'un autre type de céramique qui connaîtra un grand succès pendant tout le XVIIe s. et que l'on appelle "service vert" (A la fortune du pot 1990). Cette céramique fine et de bonne qualité sera utilisée aussi bien pour les pots culinaires que pour les céramiques de table, mais dans le dépotoir IV de Tramassac, il n'y a que des fragments de ce dernier type de vaisselle : un pichet presque entier (fig. $95 \mathbf{n}^{\circ}$ ) ) et une petite cruche avec anse en panier et un bec ponté muni d'un filtre (fig. $95 \mathbf{n}^{\circ} 2$ ).

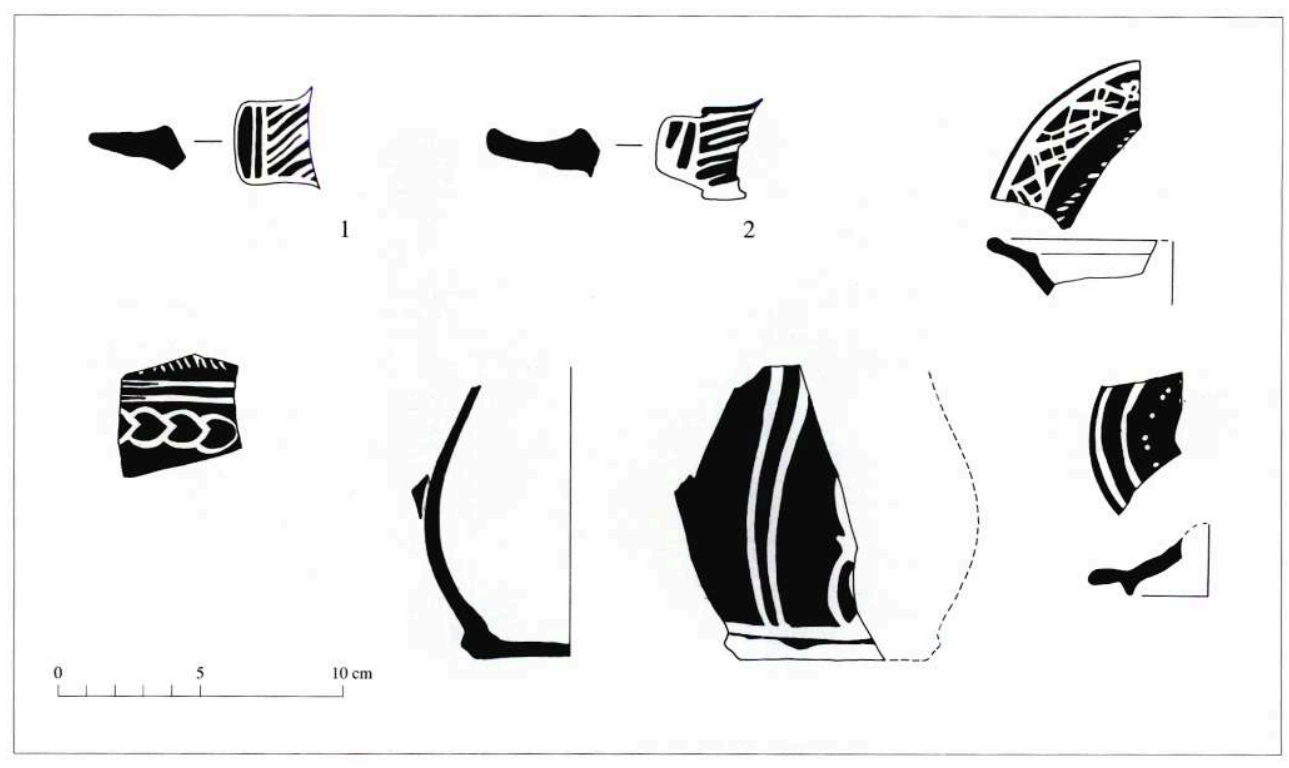

94 - Fragments décorés aux "angobes", début XVIIe s. 


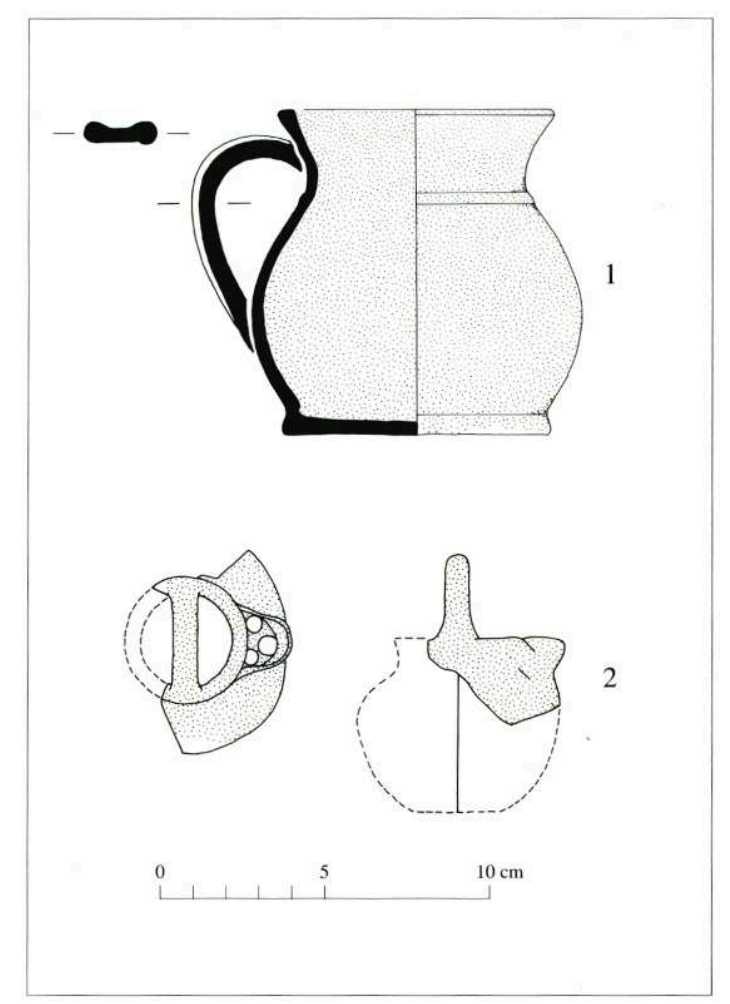

95 - Céramiques du "service vert", début XVIIe s.

\section{LES CÉRAMIQUES CULINAIRES}

130 Le grand nombre de vases destinés à la cuisson ou à la préparation des aliments compose au début du XVIIe s. une véritable batterie de cuisine en terre cuite, avec des formes de toutes les tailles (fig. 96). Leur classification amène d'abord à distinguer deux grands groupes : les céramiques à cuisson réductrice et les céramiques à cuisson oxydante.

\section{Les céramiques culinaires à cuisson réductrice}

131 Ce groupe est le moins important et ne réunit qu'un tiers environ des pots identifiables. En outre, il ne comprend que des marmites à deux anses et constitue, de ce fait, un type très caractéristique : tous les récipients, de n'importe quelle taille, ont des éléments de préhension du même type (fig. 97). Les anses, toutes de section circulaire, ont un profil anguleux, coudé ; partant de la face externe de la lèvre, elles se rabattent tout de suite sur le haut de la panse, formant une sorte d'anneau. Les rebords sont également tous du même type : légèrement évasés, ils amorcent un col court se terminant par des lèvres dont l'extrémité présente seulement quelques petites différences dans le profil, tantôt arrondi, tantôt plat. La texture de la pâte est en général assez grossière, à quelques rares exceptions près. 


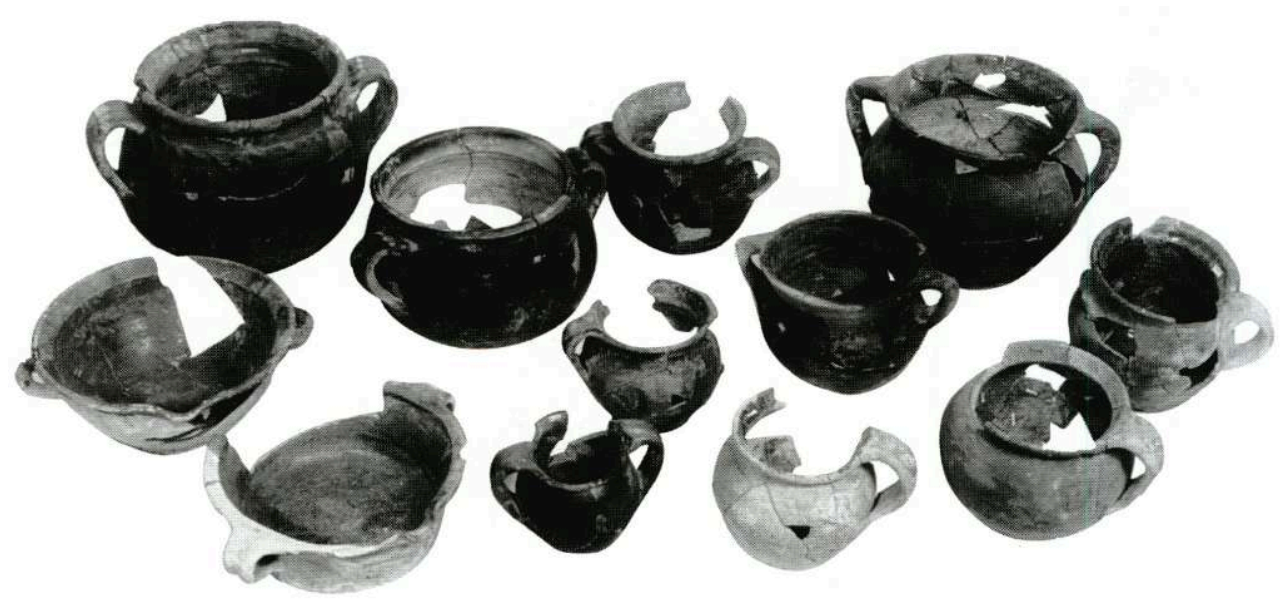

96 - Vue d'ensemble des céramiques culinaires, début XVIIe s. (Cliché P. Plattier)

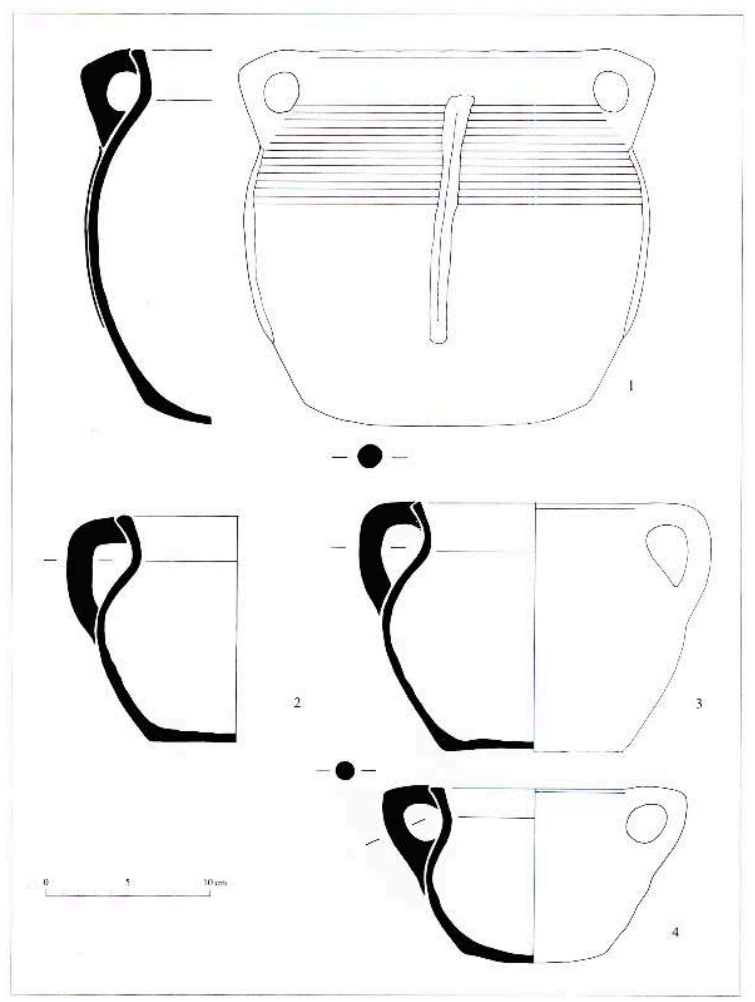

97 - Marmites à anses coudées en pâte grise, début XVIle s.

Ces marmites ne se différencient que par leur gabarit et la forme du fond. Les vases de très grande taille ont un fond bombé souligné par une arête à la liaison avec la panse et sont les seuls, en outre, à posséder une série de cannelures sur l'épaulement (fig. $97 \mathbf{n}^{\circ}$ 1). Sur ces marmites de grande taille, dont la hauteur se situe entre 20 et $30 \mathrm{~cm}$., la paroi est, de plus, renforcée par des cordons rapportés formant une crête. Ce type de renforcement a amené autrefois à distinguer ces pots sous le vocable de "pots barrés" (Renimel 1974). 


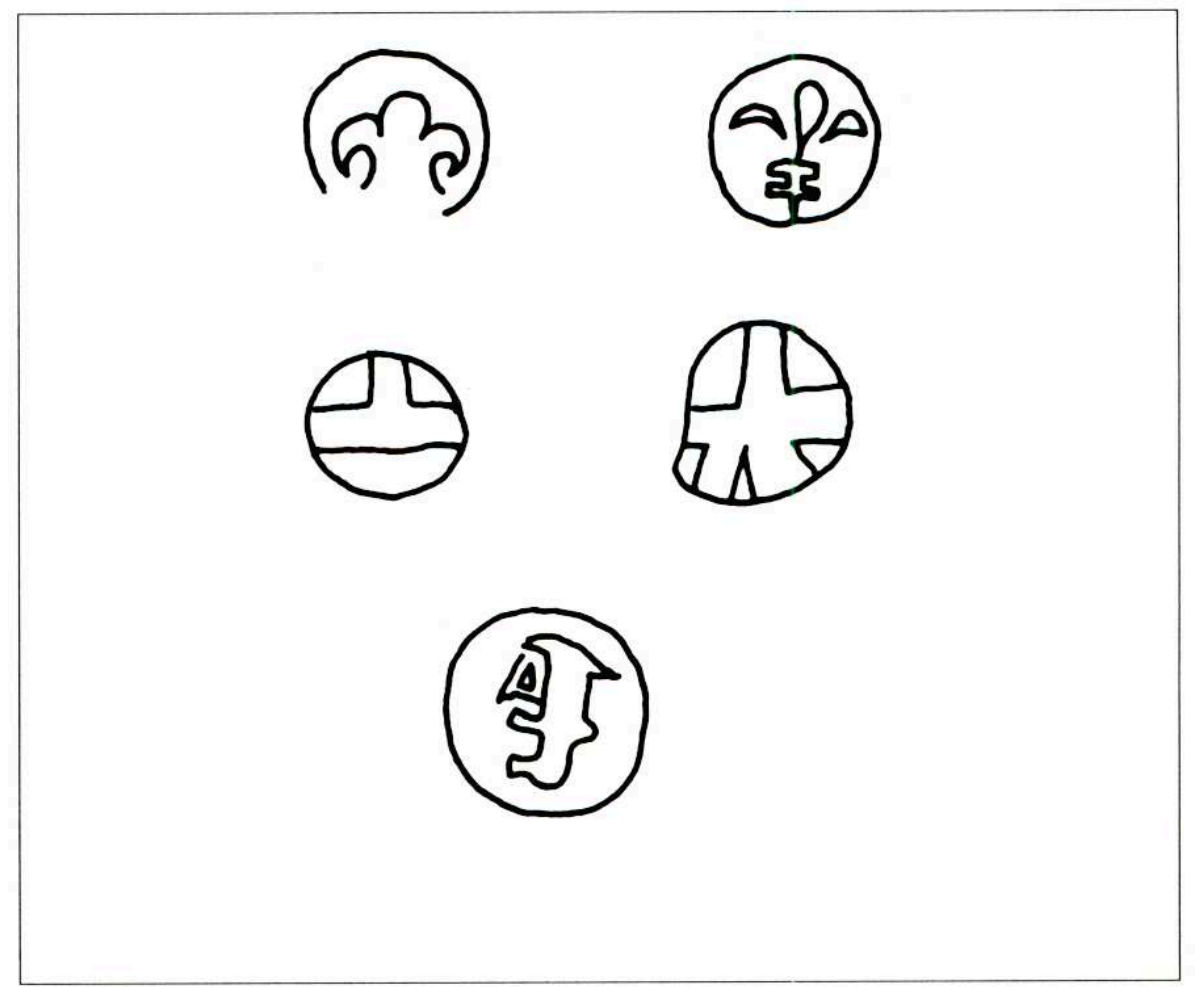

98 - Détails des marques imprimées sur l'une des anses coudées des marmites en pâte grise, début XVIIes.

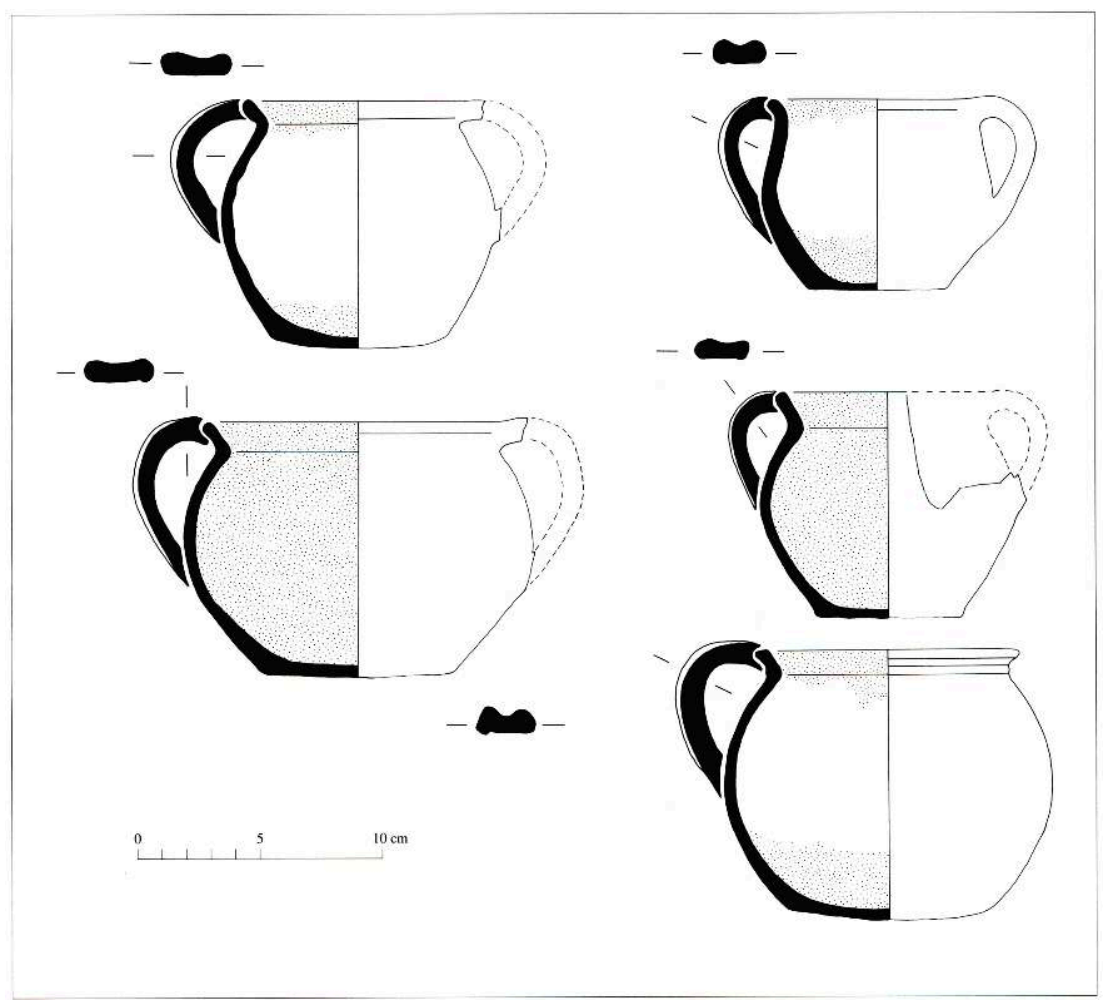

99 - MARMItes ET COQUEMAR EN PÂTE ROUge, gLAÇURÉS, DÉbUt XVIIE S. 


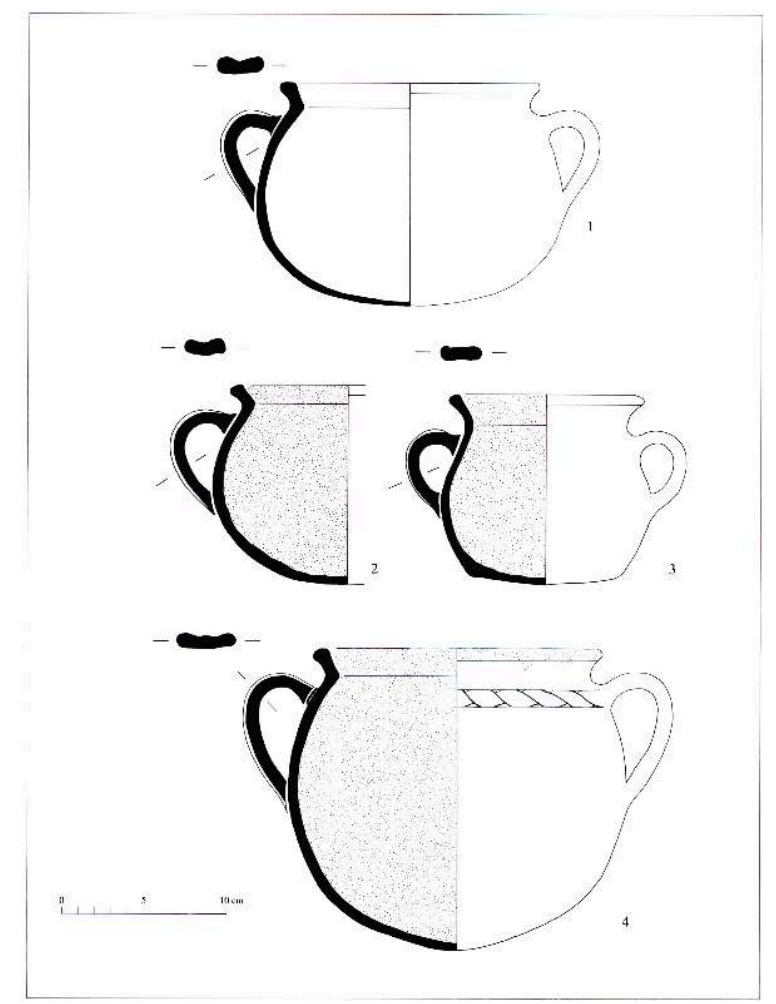

100 - Marmites en pâte rouge, glaçurées ou non, début XVIle s.

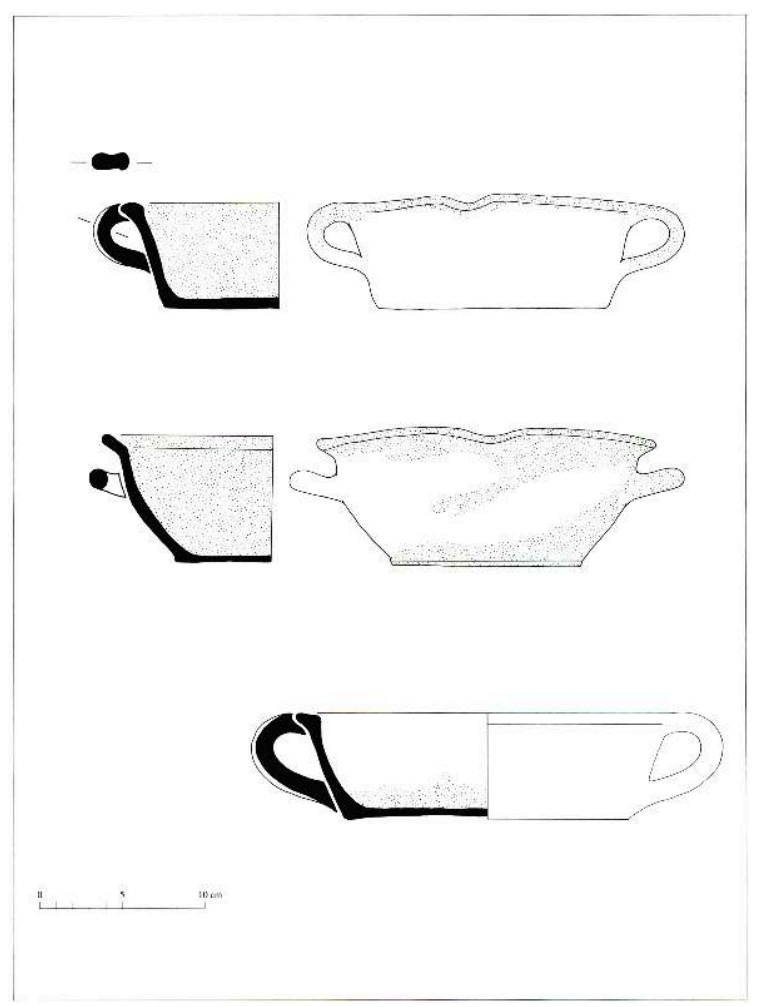

101 - Céramiques en pâte rouge glaçurées, début XVIle s.

En revanche les marmites à anses coudées de plus petite taille ne comportent ni cannelures ni cordons de renforcement. Dans ce groupe les fonds sont bombés, lenticulaires ou plats (fig. $97 \mathrm{n}^{\circ} 2$ à 4 ). 
Parfois ces marmites ont une marque sur l'une des deux anses coudées, réalisée à l'aide d'un poinçon (fig. 98). Bien que les dessins soient généralement fort mal imprimés et difficilement lisibles, trois variétés de décors ont pu être distinguées ; une fleur de lys, une composition géométrique, et sur un seul exemplaire des lignes plus complexes rappelant un monogramme.

\section{Les céramiques culinaires à cuisson oxydante}

Les récipients culinaires à cuisson oxydante constituent de loin, au début du XVIIe s., le groupe le plus important et le plus varié. Ce groupe présente une totale inversion de tendance par rapport aux périodes antérieures. Depuis leur première apparition, rarissime, au XVe s., ces pots devancent donéravant largement les productions à cuisson réductrice. Non seulement ils connaissent une très grande diffusion, mais ils se singularisent également par une large variété de formes et profils. On distingue au moins trois groupes principaux : les formes fermées avec l'attache de l'anse contre la lèvre; les marmites avec le départ de l'anse très dégagé du rebord et les formes ouvertes. Dans ces trois groupes de vaisselle culinaire, la pâte est généralement grossière, de couleur rouge ou rose sombre. Lorsque la pâte est fine, la couleur est alors rose pâle ou beige (17 formes sur 31).

Dans le premier groupe, qui se caractérise par le départ supérieur de l'anse contre la lèvre, on retrouve de petites marmites à deux anses ou des coquemars à une seule anse (fig. 99). Tous ces récipients ont un fond plat ou lenticulaire. En majorité ils sont glaçurés à l'intérieur et ce revêtement, à but exclusivement utilitaire, est soit posé sur le fond et le rebord, soit recouvre toute la surface interne. Il est toujours de couleur vert sombre.

Les pots du deuxième groupe ont l'attache de l'anse sur l'épaulement, laissant ainsi dégagé le rebord (fig. 100). Celui-ci est largement évasé. Quant au fond, il est souvent globulaire, dans un seul cas, bombé (fig. $100 \mathbf{n}^{\circ}$ 3). La glaçure de couleur vert sombre, lorsqu'elle existe, recouvre toute la surface interne. Dans un seul cas, on trouve un cordon décoratif disposé horizontalement en haut de la panse (fig. $100 \mathrm{n}^{\circ} 4$ ).

Le dernier ensemble de poteries culinaires à cuisson oxydante comprend un nombre restreint de formes ouvertes. Ces jattes, qui sont définies par leur forme basse et très évasée, ont une ouverture marquée parfois par un petit bec verseur (fig. 101). Quelques détails les différencient, comme le type d'anse ou le profil de la lèvre, la présence ou non d'un bec, mais dans l'ensemble elles semblent former un groupe homogène. La pâte, rouge, est grossière et la glaçure recouvrant entièrement ou partiellement l'intérieur est de couleur vert sombre. D'utilisations variées, ces récipients ouverts ont pu aussi bien servir comme plat de service, ou pour la préparation des aliments, mais puisqu'ils ont tous d'importantes traces de feu sur le fond, il nous a paru plus logique de les classer dans la batterie de cuisine.

Le grand nombre de formes variées qui caractérise la fin du XVIe s. ou le tout début du XVIIe s. constitue un lot intéressant pour l'histoire de l'évolution des céramiques. Cette époque apparait comme le pivot entre deux longues traditions céramiques: le monde médiéval et le monde moderne. C'est au XVIe s. que s'affirment les deux plus importantes nouveautés techniques qui domineront dans les productions postérieures : une glaçure couvrante et colorée pour les poteries les plus courantes et les vases 
culinaires, l'émail pour les céramiques de luxe. Le mobilier du dépotoir IV de l'îlot Tramassac en est un témoignage exemplaire.

\section{B. MACCARI-POISSON}

\section{LA FAUNE}

Les fouilles du secteur Tramassac ont livré un important lot d'ossements animaux. Une estimation globale fait état de plus de 50000 restes osseux, relevés, triés et enregistrés. Dans le cadre de cette étude, nous prendrons en compte environ 41000 vestiges dont les datations sont échelonnées entre le IVe et le XIVe s ap. J.-C.. Pour cette étude, inscrite dans un champ chronologique très vaste, notre principal objectif fut la recherche de séries d'observations réparties sur de longues périodes. L'attention particulière qui y fut apportée n'a pas toujours été couronnée de succès. D'importantes lacunes sont à signaler. Elles affectent tout particulièrement la première partie et la fin du Moyen Age. Pour le reste il a fallu, dans certains cas, se contenter de données éparses et discontinues.

Toute perspective historique n'a cependant pas été abandonnée. Lorsque l'état de nos sources le permet, nous nous sommes attachés à déceler les principaux traits de l'évolution des techniques bouchères, de l'économie alimentaire, de l'évolution morphologique des animaux dont les modestes vestiges osseux sont en quelques sorte "chargés". Leur déchiffrage nous a paru aussi digne de foi, et riche d'enseignements, que le dépouillement d'archives le plus minutieux ou la typologie la plus détaillée.

\section{LES ÉCHANTILLONS}

Les différentes unités stratigraphiques étudiées ont livré des échantillons dont les caractéristiques sont loin d'être homogènes. Leur importance en nombre de restes est assez inégale selon les périodes considérées (fig. 102). Les ensembles les plus importants ont été livrés par les niveaux gallo-romains tardifs : $20 \%$ des unités stratigraphiques de cette période comptent plus de 1000 restes osseux. Certains ensembles dépassent plusieurs milliers de restes. L'importance numérique des lots d'ossements décroît au fur et à mesure que l'on remonte l'échelle du temps, le Haut Moyen Age est documenté par des échantillons dont la taille, par rapport à la période précédente est généralement plus réduite. Pour la période Moderne, les échantillons de plus de 300 fragments sont inexistants. Dans la plupart des cas ils totalisent à peine 50 restes.

Cette diversité de la taille des échantillons renvoie à leur nature ainsi qu'à leur mode de dépôt. Elle n'est pas sans influer sur l'étude et conditionne des possibilités d'approches plus ou moins fines selon les échantillons disponibles.

Une quinzaine d'espèces ont contribué à la formation des échantillons de vestiges osseux. Les restes d'oiseaux, 1060 environ, figurent parmi les moins nombreux. Ils ne représentent qu'environ $0,5 \%$ de l'échantillon global, et environ autant au sein des différents ensembles chronologiques. Les restes de mammifères sont majoritaires et parmi ceux-ci les animaux domestiques prédominent largement. La chasse est attestée par la découverte d'une centaine de fragments osseux, attribués principalement aux cervidés et aux lagomorphes. Le contexte urbain, l'éloignement de la campagne sont probablement à l'origine de ce taux de prédation si faible. Ces proportions entre les 
principaux groupes d'animaux sont constantes d'une période à l'autre. Elles sont caractéristiques de l'ensemble de l'échantillon recueilli sur le secteur Tramassac. La composition du cheptel domestique est plus fluctuante. D'une période à l'autre les proportions des principaux animaux domestiques ne sont pas régulières. Les effectifs du bœuf sont en décroissance constante jusqu'à la fin du Moyen Age. A l'inverse les proportions d'ovicapridés augmentent régulièrement entre la fin de la période galloromaine et le XIXe s. Les variations traduisent des évolutions de l'orientation de l'élevage, des choix et habitudes alimentaires, de l'utilisation des produits animaux.

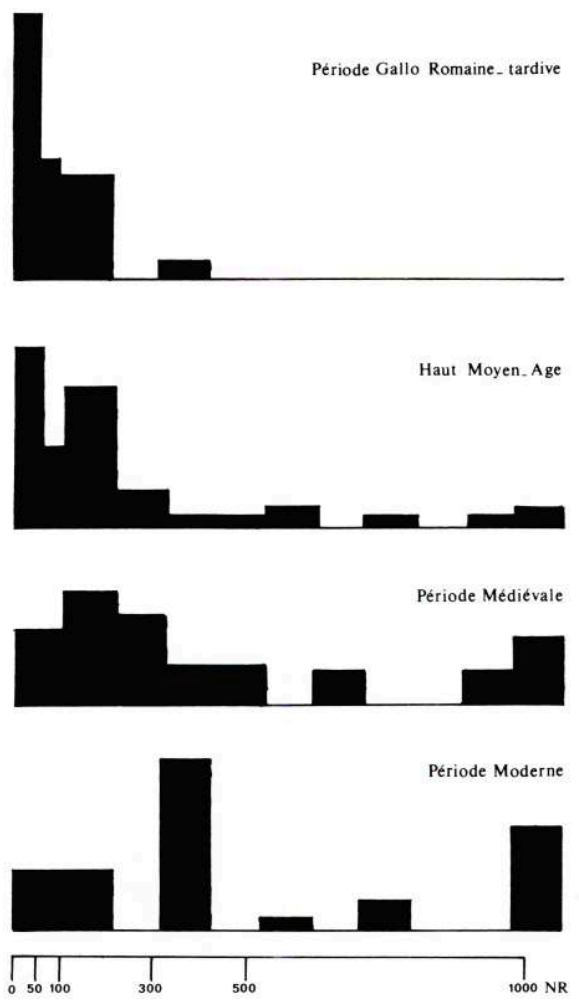

102 - Importance numérique et répartition chronologique des échantillons étudiés

145 De même le nombre de restes indéterminés au sein de chaque échantillon est assez varié. En moyenne il ne dépasse pas $40 \%$ du nombre de restes (NR) ou $25 \%$ du poids des restes (PR). C'est au sein des échantillons datés de la période Moderne que s'enregistrent les plus fortes oscillations en raison de la faible importance numérique des ensembles et de mauvaises conditions de conservation. Pour les périodes plus anciennes nous disposons d'un corpus documentaire plus fourni, tant en nombre d'échantillons, qu'en nombre d'observations. Les taux de détermination avoisinent $60 \%$ du nombre de restes et $85 \%$ du poids des restes. Ils témoignent de bonnes conditions générales de conservation.

\section{LA COMPOSITION DES ÉCHANTILLONS EN FONCTION DES DIFFÉRENTES PARTIES ANATOMIQUES}

La composition anatomique des différents échantillons est dans l'ensemble assez régulière. Quelques disparités sont à relever : l'importance des éléments du rachis de bœufs et d'ovicapridés dans les échantillons modernes ainsi que leurs faibles proportions au cours de la période galloromaine, la faible fréquence des éléments de rachis du porc, le grand nombre de fragments de crânes de porcs au cours du Haut 
Moyen Age. Cette liste pourrait être très longue. Il s'agit dans la plupart des cas d'irrégularités résultant des diverses activités dont ces rejets témoignent. Celles liées à la boucherie, à la préparation et à la consommation figurent probablement en place privilégiée.

Entre ces différents types de rejets, la distinction n'est pas aisée. Les traces enregistrées par les ossements tout au long de la chaîne d'opérations qui, de l'abattage à la consommation, transforment l'animal en aliment sont brouillées. Cette distinction est aussi conditionnée par notre connaissance de l'étal de boucherie actuel sur lequel peu d'ossements accompagnent les pièces de viande, et sur lequel les bas morceaux tendent à se raréfier.

Quelques conclusions peuvent néanmoins être tirées de l'examen de la composition des échantillons. Le relatif équilibre de la composition des ensembles gallo-romains et médiévaux est un indice en faveur d'une bonne conservation des différentes parties des animaux. Elle assure des bonnes conditions d'étude ainsi que des possibilités de comparaison. Inversement, les grandes variations caractéristiques des échantillons des XVIIIe et XIXe s. ne peuvent être considérées comme significatives, tant les ensembles sont de taille réduite.

\section{LES DÉPÔTS DE BOUCHERIE GALLO-ROMAINS}

Les échantillons datés des IVe et Ve s. ont été découverts sous forme d'importants entassements d'ossements presque exclusivement de bœufs. Certains réunissent plusieurs milliers de restes osseux, soit près d'une centaine de kilos d'ossements qui proviennent d'un Nombre Minimum d'Individus de l'ordre de la trentaine.

Une sélection assez marquée semble s'exercer lors de la formation de ces ensembles. L'étude de la composition anatomique révèle d'importants déséquilibres. L'unité stratigraphique 1121 livre, selon le mode de décompte utilisé, qu'il s'agisse du nombre ou du poids des restes, soit des éléments du rachis, soit des bas de pattes en surnombre. Dans l'ensemble 1113, il y a sureffectif de parties crâniennes et de bas de pattes. L'enlèvement et l'évacuation de ces parties encombrantes et économiquement peu intéressantes correspondent à la première étape de la préparation des carcasses. Les étapes suivantes, la découpe, la mise en parties et le débitage des morceaux, sont attestées par la fragmentation intense des os des membres et par de nombreuses traces de passage du couteau ou d'impacts de coups de couperet. Toutes les étapes du traitement boucher sont documentées (préparation des carcasses et découpe de gros, mise en quartiers, prélèvement des muscles...).

151 Les vestiges osseux relevés dans ces ensembles correspondent aux rejets de ces diverses activités de boucherie. Les restes culinaires, les rejets d'assiette n'y figurent pas.

Ces rejets de boucheries bovines sont organisés en dépôts massifs, en des lieux qui paraissent réservés à cet usage.

Dans les échantillons plus tardifs, les effets de sélection du même ordre ne sont pas absents. Il sont cependant moins systématiques. De plus les autres espèces domestiques comme le porc et les ovicapridés y figurent en place non négligeable. Il s'agit d'ensembles plus réduits qui comptent quelques centaines de restes. 


\section{LES DÉPOTOIRS MÉDIÉVAUX} dépotoirs de boucherie. La formation des échantillons résulte du rejet régulier, en quantités réduites d'ossements d'animaux. Ils correspondent de ce fait plutôt à des dépotoirs domestiques. Les échantillons ne sont plus exclusivement composés d'ossements de bœufs dont les proportions sont en recul. Cette évolution est amorcée dès la fin de la période gallo-romaine tardive. Les restes d'ovicapridés et de porcs y sont proportionnellement plus nombreux, sans qu'il soit possible d'y déceler un effet de sélection aussi marqué que dans la composition des dépotoirs de boucheries.

\section{LES ÉCHANTILLONS MODERNES}

limitée à dégager les tendances les plus marquées.

6 Les caractéristiques des différents échantillons sont en conjonction directe avec l'histoire de cette partie de la ville. L'évolution du site est marquée par une progressive raréfaction des dépôts détritiques, probablement due à l'installation des jardins du Doyenné, au resserrement du tissu urbain, à l'évolution du statut social du quartier.

\section{LE CHOIX DES ESPĖCES CONSOMMÉES ET LEUR DÉCOUPE BOUCHÈRE}

Les proportions des différentes espèces consommées sont assez fluctuantes (fig. 103). Largement prédominante dans les échantillons du IVe et Ve s., la place du bœuf est dès le VIe s., nettement plus réduite. Elle est en décroissance constante durant la période médiévale et moderne. Dans les échantillons d'ossements animaux des sites GalloRomains de la vallée du Rhône (Cl. Olive, communication orale), le bœuf occupe aussi la place la plus importante. Ce rôle réduit des ovicapridés trouve des équivalents sur les sites du Nord de la France (Vadet 1981). Dans la partie méridionale, les effectifs des petits ruminants sont nettement plus importants et avoisinent $72 \%$ du nombre de restes (Jourdan 1976). A Lyon, ce n'est qu'au cours du VIe s. qu'une place plus importante semble réservée à ces animaux. 

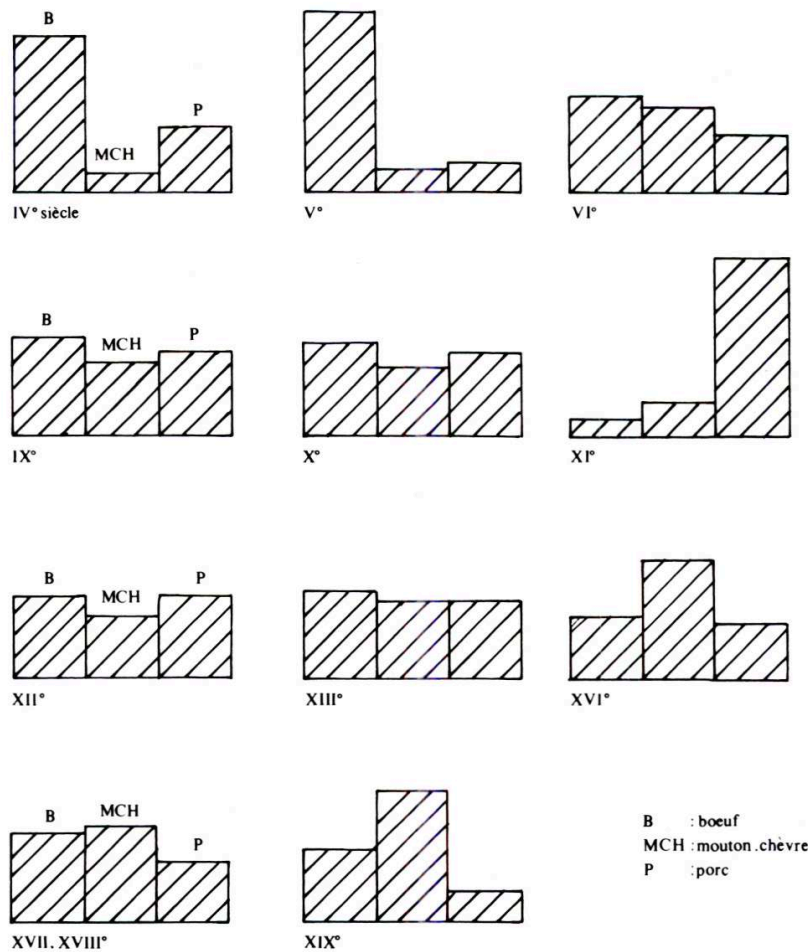

XVII XVII'

$\mathrm{xIX}$

$\mathrm{P} \quad$ : porc

103 - Variations des proportions relatives du Nombre de Restes de différentes espèces domestiques

Dès le IXe s., les principales espèces domestiques ont livré des restes en proportions presque équilibrées. Le premier rang revient au bœuf suivi, par ordre d'importance, du porc et des ovicapridés jusqu'au XIIIe s. L'évolution parait surtout marquée par l'accroissement des proportions de porcs. Cette observation concorde avec les données livrées par d'autres sites: la Charité-sur-Loire (Auduin et Marinval-Vigne 1987), et Haithabu (Reichstein 1974). A partir du XVIe s. et jusqu'au XVIIIe s., la composition des échantillons évolue au profit des petits ruminants largement prédominants dans la plupart des échantillons, alors qu'une place non négligeable semble réservée au bœuf.

\section{LE BCEUF}

C'est l'espèce qui a livré le plus de vestiges. Durant la période gallo-romaine tardive, le bœuf est largement prédominant. C'est pendant le Ve s. que ses proportions sont les plus élevées au sein des échantillons (fig. 104) : ses restes représentent $92 \%$ du poids des restes et $78 \%$ du nombre de restes. Le début de l'ère médiévale est caractérisé par un recul de l'élevage bovin. Mise à part une forte baisse pendant le XIe s., cet élevage est marqué par une certaine stabilité. Il ne diminue que faiblement, de l'ordre de $10 \%$, durant la période médiévale. Pendant la période moderne, la courbe enregistre une légère reprise des effectifs bovins. L'élevage du porc semble le moins développé. Les fluctuations de la place des différentes espèces indiquent d'une part l'évolution de la consommation des Lyonnais. Elles sont d'autre part à mettre en rapport avec une évolution des utilisations et du rôle des animaux. 


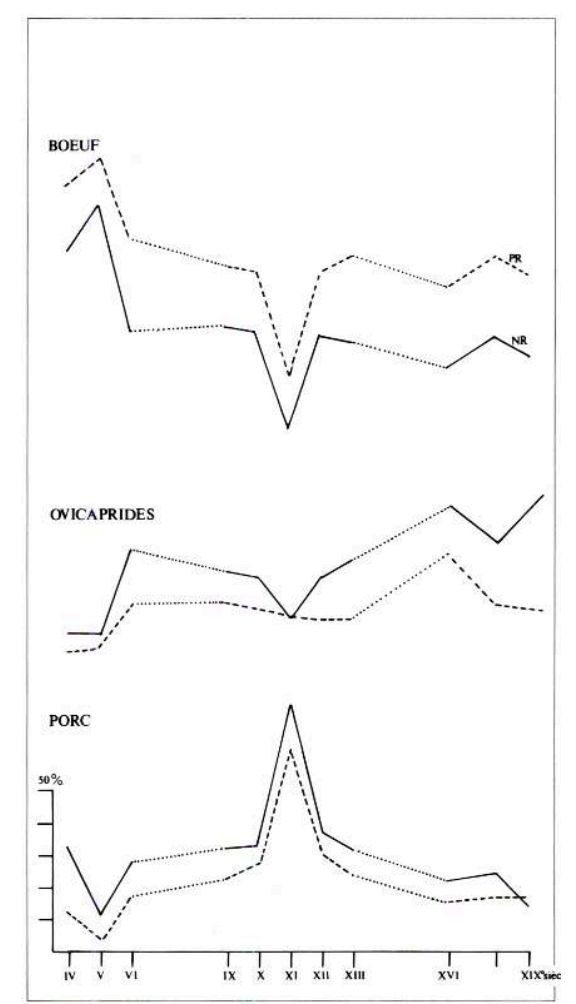

104 - Evolution des proportions relatives du Nombre de Restes et du Poids des Restes des différentes espèces domestiques

Les élevages bovins médiéval et moderne semblent procéder et se maintenir selon des proportions établies dès la fin de la période Romaine. Quelques "accidents" jalonnent cette évolution. Il s'agit des effectifs très élevés des IVe et Ve s., et ceux très faibles du XIe s. qui sont dus, dans le premier cas à la nature particulière du mode de dépôt et, dans le second cas, à la faible représentativité des échantillons.

Les importants échantillons gallo-romains nous livrent de la découpe bouchère une image très homogène. Plusieurs étapes peuvent être discernées: la préparation des carcasses par l'enlèvement des parties crâniennes et des bas de pattes, le débitage et la découpe des carcasses, le prélèvement des muscles et la récupération de la moelle. L'utilisation d'un outil en métal, assez lourd, du type du couperet, est attestée tout au long de la chaîne du traitement boucher. La colonne vertébrale est détachée par coups portés le long des corps vertébraux, brisant les apophyses transverses (fig. 105). Le train de vertèbres est débité en segments d'environ cinq vertèbres. La technique de la fente des vertèbres en deux parties symétriques n'est que rarement observée. Elle s'applique le plus souvent aux vertèbres cervicales. D'une manière générale, les vertèbres sont en quelque sorte "réduites": les apophyses transverses et épineuses étant tranchées. Cette dernière opération semble effectuée sur des tronçons de vertèbres en connexion que l'étude a dans certains cas réussi à reconstituer. Les côtes ont vraisemblablement été isolées par des coups rasant soit l'articulation costale, soit le corps des vertèbres. La suite du traitement des carcasses consiste essentiellement en une mise en parties assez grossière. Toutes les relations articulaires sont défaites de façon assez brutale, à coups de couperet affectant particulièrement les parties saillantes (ulna et calcanéum). La découpe des membres se réduit à une désarticulation violente, mais sans précision, des différentes parties. Il en va différemment des parties charnues. La viande est prélevée à l'aide d'un couteau, secondé dans certains cas par 
l'utilisation du couperet. Les os sont dans la plupart des cas fracassés afin de récupérer la moelle. La fragmentation très poussée des diaphyses et les nombreux stigmates du passage du couteau nous renvoient à ces diverses opérations.

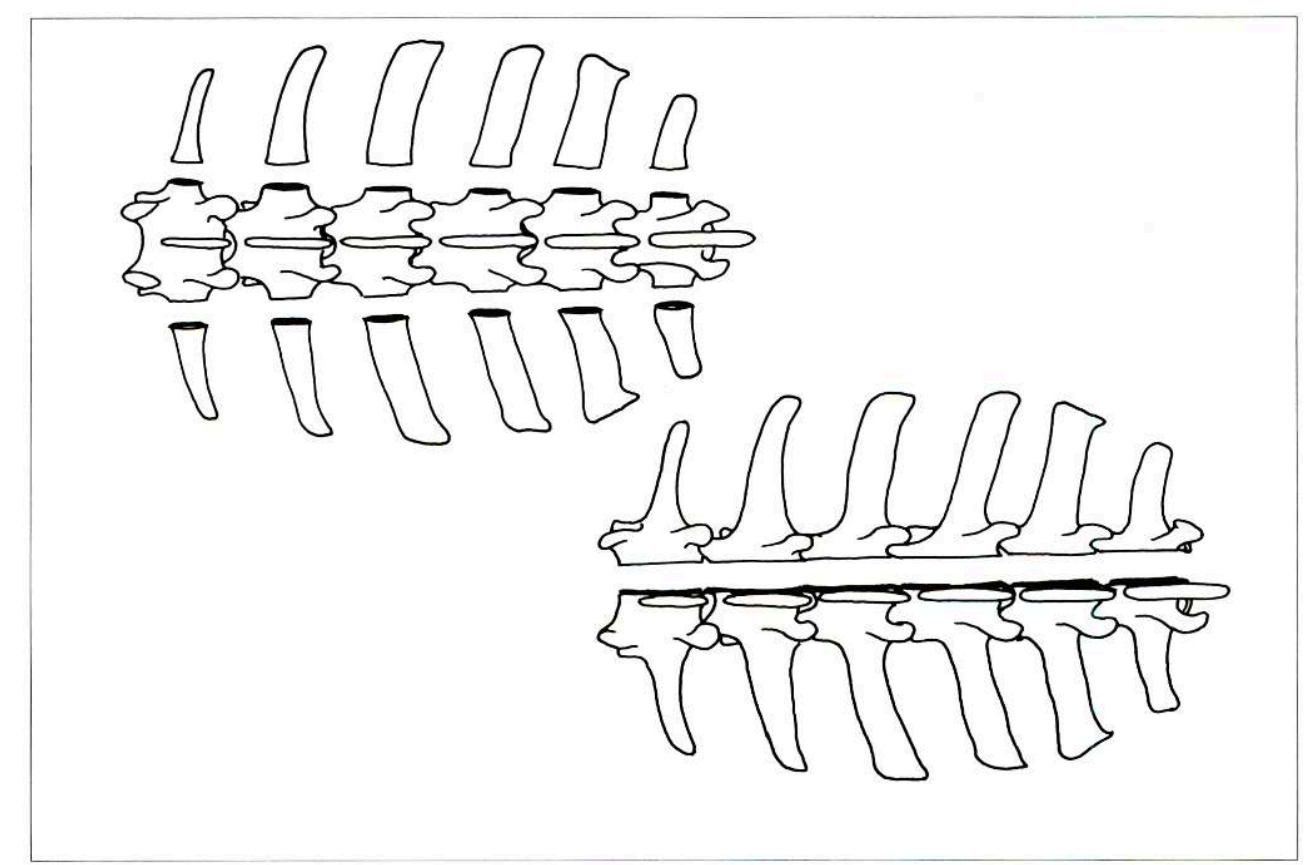

105 - Schéma de découpe des vertèbres selon la technique du prélèvement du train de vertèbres par coups de tranchet portés de chaque côté et selon la technique de la fente sagittale

Durant le Moyen Age, deux techniques de découpe de gros ont cours, le prélèvement du train de vertèbres selon la méthode suivie par les bouchers gallo-romains, et celle de la fente longitudinale telle qu'elle est pratiquée en boucherie moderne. L'adoption de cette nouvelle technique est observée de façon très discontinue. La découpe de la carcasse en tronçons transversaux semble largement prévaloir et ce jusqu'au XVIIe s. La technique de la fente médiane semble surtout utilisée pour la préparation de pièces précises.

Dans plusieurs ensembles, et dès le Xe s., ce traitement est réservé aux vertèbres cervicales. Il intervient probablement dans une étape plus avancée du traitement boucher et reste limité à la préparation des pièces de collier.

Cette pratique va dans le sens d'un plus grand morcellement et d'une diversification de la découpe. Ces caractéristiques semblent aussi prédominer dans la suite des opérations du partage des animaux. On observe en effet un fractionnement intense des différentes parties. La désarticulation pratiquée à l'aide du couperet est précédée et guidée par des incisions au couteau qui dégagent les zones articulaires. Elle est de ce fait nettement plus précise. Le prélèvement des chairs semble plus soigneux. La décarnisation est essentiellement effectuée au couteau; le couperet n'intervenant plus à ce stade de la découpe. Tous les os, y compris ceux des parties les moins prisées comme les métapodes, sont systématiquement fractionnés dans le but d'en récupérer la moelle, alors que dans les échantillons gallo-romains, les bas de pattes, mais d'autres parties également, sont souvent entières, indemmes de toute fragmentation.

L'état lacunaire de nos données ne permette pas de suivre l'évolution des techniques bouchères vers la fin de la période médiévale. Des observations concernant les XIVe, XVe et XVIe s. font particulièrement défaut. 
$166 \mathrm{Au}$ cours du XVIIe s., le partage de la carcasse par un tronçonnage médian semble déjà bien institué. Toutes les vertèbres sont, d'un bout à l'autre de la colonne, systématiquement tranchées en deux. L'adoption de cette technique de partage est significative d'une modification du schéma de découpe. Elle conditionne un choix de morceaux plus large, ainsi que des gestes nouveaux. Cette diversification concerne essentiellement les pièces du squelette axial, c'est-à-dire ceux de la colonne vertébrale et du rachis. D’après nos données une telle modification n'est pas sensible pour la découpe des membres.

\section{LE MOUTON ET LA CHÈVRE}

167 L'élevage de ces deux espèces connaît une importance croissante dès la fin de l'Empire Romain. Dans les dépotoirs de boucheries galloromains, les vestiges de petits ruminants sont assez discrets, les proportions moyennes y sont de l'ordre de $25 \%$ du NR et $13 \%$ du PR. Dès le VIe s., leur élevage est en essor. C'est à la fin du Moyen Age qu'il est le plus important.

168 La découpe des petits ruminants est dans ses lignes les plus générales similaire à celle des bovidés. Au cours des IVe et Ve s., le train de vertèbres est extrait de la carcasse et ne fait pas l'objet d'une fente médiane. Dans quelques cas, les morceaux de colliers ont été tranchés en deux.

169 Les crânes, débarrassés des chevilles osseuses à coups de couperet ont été fendus en deux, ou décalottés. Cette série d'opérations évoque la consommation de la cervelle et de la tête.

170 La découpe de la carcasse dans le sens longitudinal semble s'imposer assez tôt. Elle est observée dès le XIIIe s., soit plus tôt que dans le cas du bœuf. Dès cette date, la première technique semble abandonnée. Les moitiés de rachis sont par la suite débitées transversalement en morceaux dont la taille tend à se réduire au fil du temps.

171 La fréquence des coups de couperet perpendiculaires au corps des vertèbres est au cours de la période médiévale de plus en plus élevée. Les côtes restent attenantes aux vertèbres, formant des pièces du type carré-de-côtes, dont l'évolution extrême est la côtelette individuelle. Le bas des côtes, moins apprécié, est séparé par un coup de tranchet.

172 La préparation des différentes parties est marquée par la même tendance. Les parties les plus charnues font l'objet d'une découpe assez soignée pour laquelle, dès la période gallo-romaine, le couteau semble supplanter le couperet. Les os ont ensuite subi une fragmentation intense dans le but de récupérer la moelle. La mise en pièces à l'aide d'un outil lourd est plus fréquente pour la préparation des parties basses dépourvues d'importantes masses musculaires. Les bas de pattes (métapodes et phalanges) n'ont pas été dédaignées. Leur consommation est largement attestée. Elle a laissé des traces caractéristiques : extrémités carbonisées, incisions sur la diaphyse...

L'évolution est marquée par une mise en pièces de plus en plus poussée des parties. Les os sont débités en segments dont la taille est de plus en plus réduite. Cette évolution indique une sélection de plus en plus active et précise des pièces en fonction de leur intérêt et de leur valeur bouchère. Leur fragmentation est de plus en plus poussée au cours du Moyen Age, les os du coude et du jarret enregistrent de manière particulièrement nette cette tendance. Elle constitue le principal trait distinctif entre la boucherie gallo-romaine et de celle des périodes médiévale et moderne. 


\section{LE PORC}

Le porc est l'animal de boucherie par excellence. Son élevage est exclusivement orienté vers cette production. Dans les troupeaux gallo-romains composés en grande partie de bovins sa place est des plus réduites (fig. 102 et 103).

C'est au cours de la première partie du Moyen Age que ses effectifs sont les plus importants. Ils enregistrent un recul régulier et constant entre le XIIe et le XVIIII s.

6 Le partage de la carcasse, de laquelle le train de vertèbres est préalablement extrait, est attesté dans les échantillons gallo-romains. Cette technique semble avoir cours pendant tout le Moyen Age. La fente médiane semble adoptée assez tardivement.

Il faut attendre le XVIIIe s. pour l'observer. Ainsi que dans le cas du mouton, les pièces de collier ont souvent été fendues en deux, et ce dès la période gallo-romaine.

La fente médiane est aussi pratiquée pour ouvrir le crâne et trancher la symphyse mandibulaire. Cette méthode est utilisée conjointement à celle qui consiste à fractionner les mandibules au niveau du diastème, perpendiculairement à la symphyse. Elle n'est pas corollaire de la pratique de la fente médiane de la carcasse.

En boucherie gallo-romaine, la mise en pièces des différentes parties est réalisée au tranchet. Elle consiste essentiellement en une désarticulation et une fragmentation des morceaux les plus riches en viande (jambon et épaule). Les pièces de l'avant-bras et du jarret restent souvent intactes de toute fragmentation, de même que les pieds. C'est du moins ce que suggèrent les quelques os (radio-ulnaires, tibias et métapodes) restés entiers. Ces morceaux semblent préparés et arrivent sur la table tels quels. Les observations sont transposables à la boucherie du haut Moyen Age. Dès le XIIIe s., la tendance à une fragmentation plus intense est très accusée. A l'exception des bas de pattes, tous les os sont intensivement réduits en morceaux. La boucherie du porc semble caractérisée par une évolution analogue à celle des ovicapridés. La tendance au morcellement et à l'intensification de l'exploitation des différentes parties est des plus manifestes.

\section{LES ÉQUIDES ET LE CHIEN}

D'autres espèces domestiques, comme les chevaux et les chiens ont fait l'objet d'une exploitation bouchère et ont été consommées.

1 Les ossements d'équidés sont, dans la majorité des cas, marqués de traces de découpe. La consommation de la viande de cheval est attestée dès la période gallo-romaine et perdure jusqu'à la période moderne. Elle ne concerne pas tous les sujets, elle est néanmoins observée régulièrement et semble assez courante. La plupart des traces correspondent à des activités de désarticulation et se présentent sous forme de fines incisions. Des impacts de coups de couperet, des zones tailladées violemment signalent un traitement plus expéditif.

La rareté des ossements d'équidés représente une limite importante de l'étude de la consommation et des autres utilisations de cet animal. La production bouchère n'exclut pas celle de l'énergie, de la traction. La présence de plusieurs types d'équidés, souligne le rôle important de ces animaux pour le transport, les déplacements, et l'acheminement. Signalons que la consommation, ultime utilisation de l'animal, semble aussi concerner les équidés de petite taille comme l'âne et le mulet. Il est impossible 
dans l'état actuel de notre corpus documentaire de préciser les règles de sélection et de gestion de ces animaux.

La découpe et la consommation de chiens est attestée de façon plus ponctuelle sur le site.

En contexte gallo-romain (US 1098) trois demi-mandibules de chiens présentent des traces de débitage au tranchet. Un radius est marqué d'incisions dues au prélèvement de la viande. La localisation de ces traces évoque plutôt l'écorchement que la consommation. Elles sont en effet situées sur des parties peu charnues.

Dans les niveaux médiévaux (US 710 et 678) les restes de quatre individus découpés ont été recueillis. Un crâne présente des fines incisions, un autre a été débité plus grossièrement à l'aide d'un instrument lourd. De nombreuses traces de découpe ont été relevées sur la plupart des os des membres et des ceintures. Les éléments du rachis ne présentent pas de tels stigmates. La marque du couteau est souvent imprimée très profondément dans l'os, signalant la maladresse d'un praticien peu formé à ces opérations, ou le manque d'habitude d'un boucher ordinairement confronté à la découpe des carcasses de bœufs, de porcs et de moutons.

Un nombre réduit d'animaux a subi l'écorchement, la désarticulation et le prélèvement des chairs. Leurs restes ont été rejetés simultanément. L'étude ostéologique a permis la restitution des liaisons articulaires de plusieurs parties. Le rejet de plusieurs carcasses dépecées et dont une partie au moins des muscles a été prélevée, ne correspond pas au traitement habituel des autres espèces de boucherie. De ces observations, se déduit le caractère fermé des échantillons dont il est question, ainsi que le rôle limité de la boucherie de ces espèces.

\section{LE CHOIX DES ANIMAUX EN FONCTION DE L'ÂGE ET DU SEXE}

L'étude des structures d'abattage nous renseigne sur le mode de gestion du cheptel et les règles de sélection des animaux ; croisée avec celle de la composition des troupeaux selon les sexes, elle permet de caractériser la nature de l'intervention humaine et de déceler les différentes utilisations des animaux. Ces investigations sont nécessairement à fonder sur un grand nombre d'observations, dont nous ne disposons que de manière discontinue et irrégulière. L'étude des âges d'abattage (Barone 1976) est effectuée d'après le degré d'épiphysation des os longs. Les vestiges d'animaux en bas âge, fragiles, y sont presque obligatoirement sous-représentés. Ces limites, inhérentes, aux vestiges disponibles restreignent de façon drastique le champ et la portée de l'étude.

\section{LE BCEUF}

Une dizaine d'échantillons ont livré des vestiges osseux en assez grand nombre pour établir des courbes d'abattage. Les ensembles recueillis dans les dépotoirs galloromains y figurent en bonne place. Les histogrammes des âges d'abattage sont des plus homogènes. Les animaux sont tués vers trois ans et au-delà. Les bêtes de moins de 24 mois semblent épargnées. L'abattage concerne de façon préférentielle les animaux âgés d'environ 3-4 ans. Quelques individus peu nombreux sont gardés au delà de ce seuil (fig. 106).

Une composition des troupeaux largement dominée par des animaux jeunes et subadultes, dans laquelle la place des animaux adultes et séniles est très réduite, 
correspond à une gestion essentiellement orientée vers la boucherie. Cette utilisation quasi exclusive des animaux conditionne le type même des rejets; le terme "dépôts de boucherie" paraît dans ce cas particulièrement justifié.

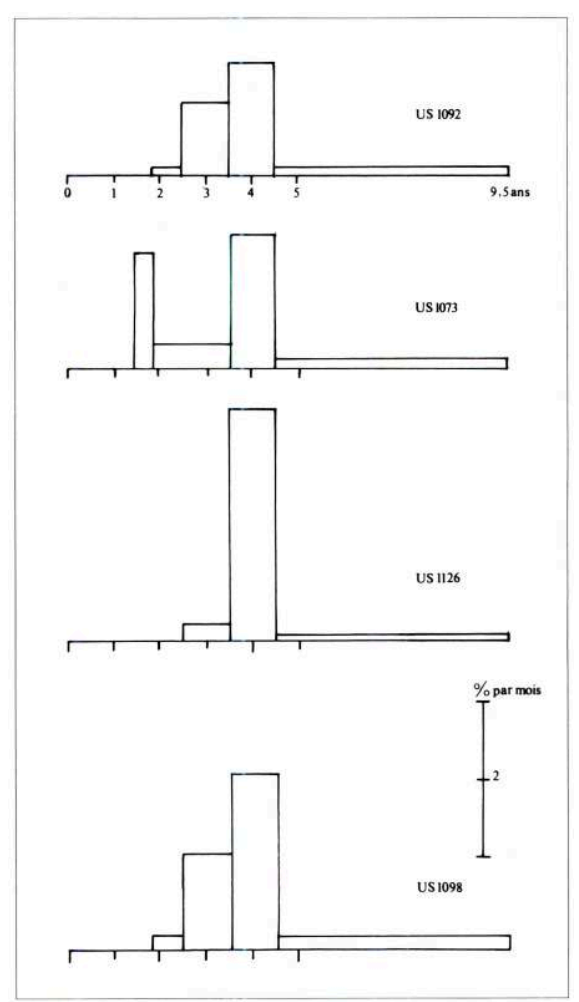

106 - Courbes d'abattages des bœufs établies d'après le degré d'épiphysation des os longs. Période gallo-romaine

Le choix des animaux en fonction de leur sexe permet de préciser davantage la composition du troupeau bovin. Il peut être mis en évidence par l'intermédiaire de l'étude biométrique. Les proportions des métapodes enregistrent de façon assez précise les effets du dimorphisme sexuel. De plus, cette partie du squelette figure parmi les mieux conservées. Plusieurs échantillons gallo-romains ont livré des métacarpes et des métatarses en assez grand nombre. Les diagrammes de dispersion des mesures sont structurés en trois groupes : le premier, assez important, correspond probablement aux animaux castrés. Il réunit plus de $70 \%$ de la population. Le second, celui des vaches, ne constitue qu'environ $25 \%$ des effectifs. Le dernier, celui des taureaux est avec ses $5 \%$ d'effectifs, le plus réduit (fig. 107).

191 Le choix des animaux parait particulièrement ciblé sur les animaux castrés. Leur abattage vers 3-4 ans, intervient au moment où leur rendement en viande est le plus important. La pratique de la castration, par les modifications qu'elle entraîne, contribue à l'amélioration du rendement boucher. L'élevage bovin, d'après les tendances qui se dégagent de l'étude de ces données, est principalement orienté vers la production d'animaux de boucherie à rendement élevé. L'équilibre des sexes tel qu'il existe à la naissance n'est pas respecté.

Cette sélection n'est pas sans influer sur la nature des dépôts de boucherie. Elle en constitue le premier et principal agent. Elle livre à la boucherie des animaux de sexe identique, de même âge et probablement de même conformation. Cette uniformisation est prolongée par celle du traitement boucher, et des types de rejets. Au fil des 
sélections successives, la boucherie antique nous apparaît particulièrement organisée et très spécialisée.

Pour la période médiévale, les données disponibles concernent surtout les IXe et XVIIe $\mathrm{s}$

es courbes d'abattage du IXe s. attestent une certaine continuité avec la période précédente. Les premières classes d'âge ne subissent aucun prélèvement (fig. 108). L'abattage concerne surtout les animaux âgés de deux à cinq ans. Il n'intervient plus à une étape précise de la croissance des animaux, il est au contraire réparti régulièrement sur plusieurs classes d'âge. Des pics d'abattage importants et très localisés ne sont pas décelables sur les courbes médiévales. La gestion du cheptel bovin évolue vers un choix ciblé de manière moins précise. Il s'agit d'une sélection moins active des animaux. Elle relève d'une utilisation diversifiée des différentes productions d'origine animale. Les animaux ne fournissent pas uniquement leur viande, les autres ressources, lait, énergie, semblent exploitées. Un abattage échelonné entre les différents âges, permet de les faire valoir de manière plus intensive. Cette évolution vers une gestion plus diversifiée est sensible sur toute les courbes du début du Moyen Age. A l'élevage spécialisé d'animaux de boucherie strictement sélectionnés, succède un élevage polyvalent régi à la fois par les besoins de l'alimentation, et l'utilisation de l'énergie et des autres matières premières animales, (lait, laine...).

L'étude de la composition des troupeaux médiévaux en fonction du sexe des animaux est confrontée à la difficulté de réunir des os entiers d'animaux en nombre suffisant. L'établissement d'un diagramme de dispersion de dimensions d'os longs a nécessité le recours aux données d'échantillons non strictement contemporains dont les datations s'échelonnent entre le IXe et le XVIIe s. (fig. 109). Les conclusions sont à considérer comme hypothétiques et provisoires.

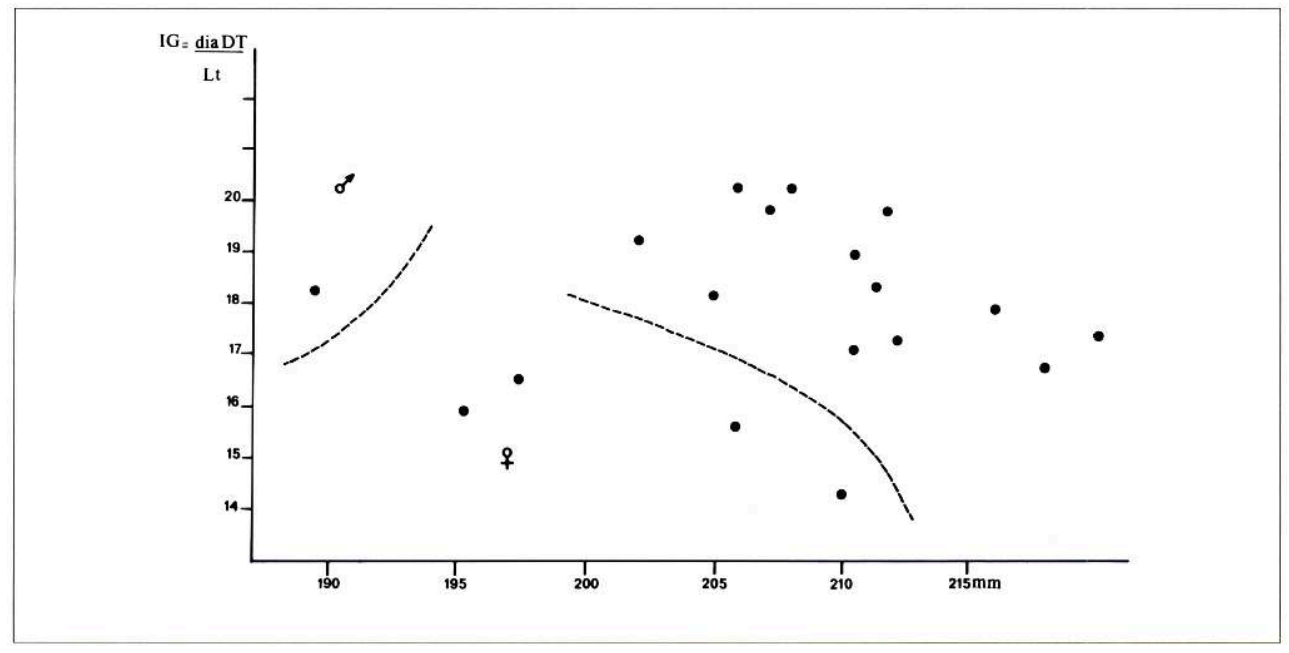

107 - Diagramme de dispersion des mesures de métacarpes de bœufs. Période gallo-romaine 


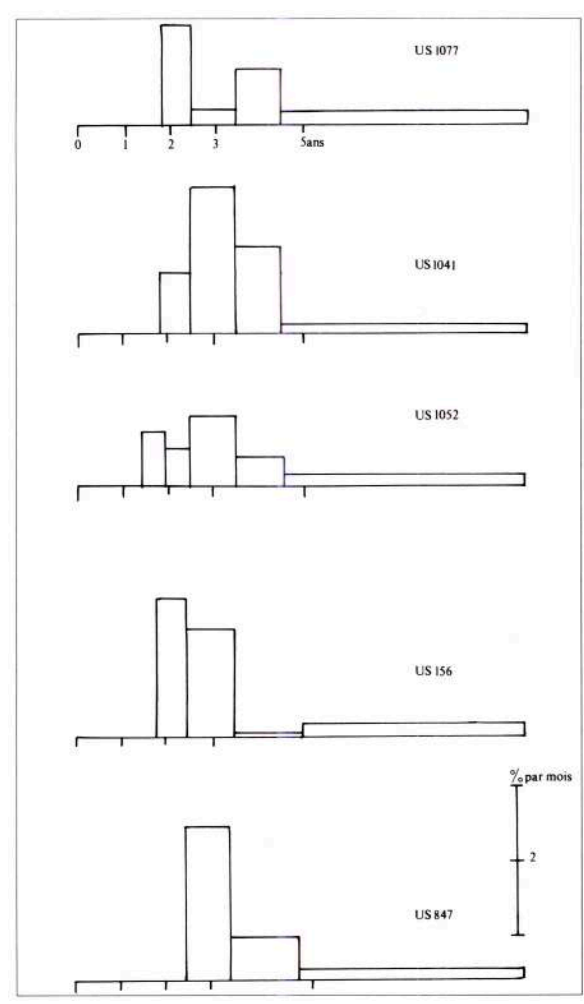

W108 - Courbes d'abattage des bœufs établies d'après le degré d'épiphysation des os longs. Période médiévale

La composition des troupeaux médiévaux procède dans ses lignes les plus générales d'un fond commun avec la période précédente. Dans les troupeaux médiévaux, les proportions entre les vaches, taureaux et bœufs paraissent presque inchangées. Celles des animaux castrés enregistrent un léger recul. Les taureaux y sont un peu plus nombreux. Cette composition s'accorde avec l'utilisation des différents produits de l'élevage. La présence de mâles en nombre non négligeable est à mettre en rapport avec leur utilisation au trait et à la boucherie. Les vaches ont probablement aussi servi à ces usages, elles fournissent en plus le lait et assurent la reproduction. La place importante réservée aux bœufs et aux taureaux, la proportion croissante d'animaux ayant dépassé la maturité, sont les principaux indices d'une évolution de la gestion et de l'utilisation des animaux.

\section{LES OVICAPRIDÉS}

197 Cette dénomination correspond à un artifice commode pour désigner le mouton et la chèvre. La distinction entre ces deux espèces sur la base d'ossements fragmentés n'est pas chose aisée. La détermination spécifique est basée sur l'observation morphoscopique complétée par une étude biométrique de certaines parties du squelette; chevilles osseuses, os longs. Les chevilles osseuses sont de formes différentes, selon qu'il s'agit d'un mouton ou d'une chèvre, et leurs proportions sont très variables selon qu'il s'agit d'un bouc ou d'une chèvre, d'une brebis ou d'un bélier. C'est sur la base de ce double critère de distinction qu'il est possible de préciser en partie la composition des échantillons de petits ruminants. 


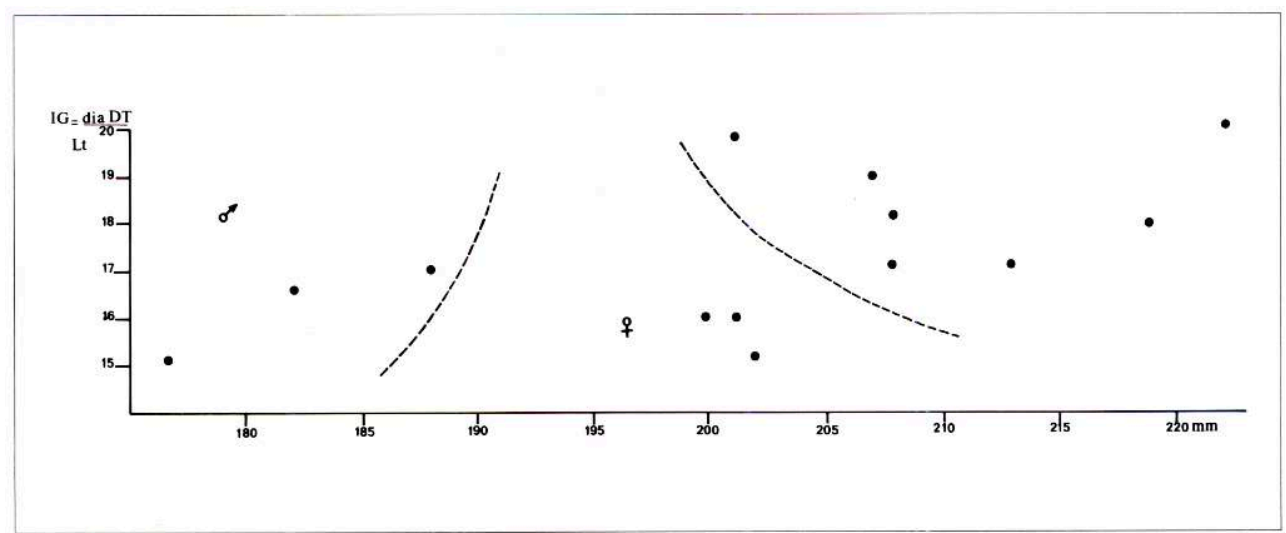

109 - Diagramme de dispersion des mesures de métacarpes de bœufs. Période médiévale

Dans les échantillons de la période galloromaine tardive, les proportions entre ovins et caprins sont équilibrées. Durant la période médiévale, les effectifs ovins semblent plus développés. Ils représentent environ $70 \%$ des individus. La diminution des effectifs caprins révèle probablement une modification des traditions pastorales. L'élevage de la chèvre semble au cours de la période médiévale moins adapté aux divers besoins.

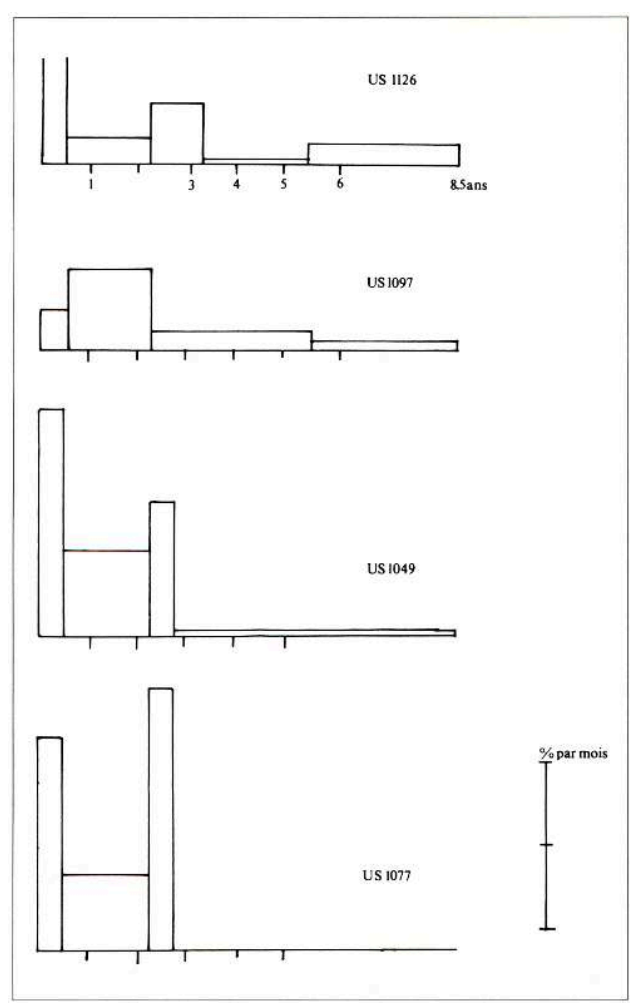

110 - Courbes d'abattages des ovicapridés établies d'après le degré d'épiphysation des os longs. Période gallo-romaine 


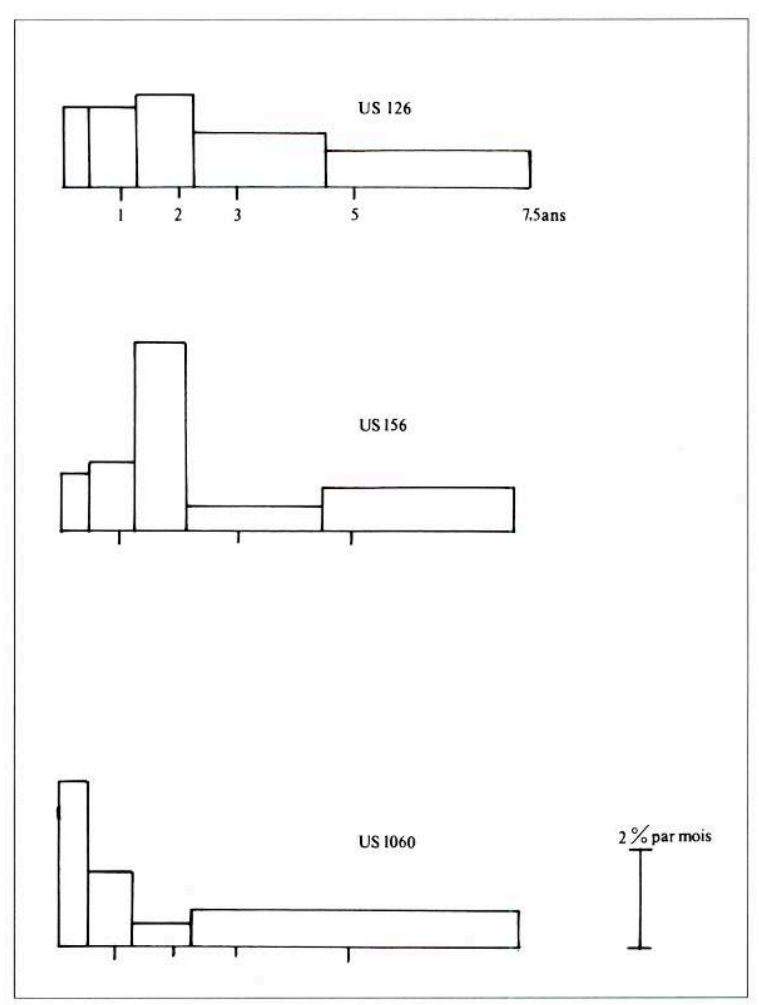

111 - Courbes d'abattage des ovicapridés établies d'après le degré d'épiphysation des os longs. Période médiévale

De même la gestion de ces animaux est sujette à d'importantes variations. L'unique courbe d'abattage (fig. 110) dont nous disposons pour la période gallo-romaine tardive atteste un abattage intensif des jeunes. L'essentiel des prélèvements est effectué entre 6 mois et 2 ans. Des animaux adultes sont maintenus en proportions moins importantes dans les troupeaux. Au-delà du seuil de la maturité, leur abattage est pratiqué assez tardivement.

Le système de sélection est transposable aux troupeaux médiévaux (fig. 111). Sur certaines courbes du début de cette période on enregistre cependant une intensification du mode d'exploitation des troupeaux.

Les agneaux âgés d'environ six mois sont sacrifiés en proportions plus importantes. L'abattage d'un grand nombre d'animaux de moins de deux ans est maintenu. Les dernières classes d'âge subissent une décroissance régulière des effectifs. Cette intensification de la régulation des troupeaux d'ovicapridés est aussi sensible sur le courbe datée du XVIIIe s. Elle s'exerce cependant d'une manière différente. L'abattage des agneaux est pratiqué en proportions nettement diminuées. Les animaux d'environ deux ans subissent l'essentiel des prélèvements. Au-delà de ce seuil et jusque vers cinq ans, on observe une diminution des activités d'abattage. Il s'agit d'un élevage régi par l'élimination des bêtes de réforme, et par l'intégration des jeunes de l'année. Il relève d'une gestion soucieuse de maintenir et d'assurer le fonctionnement du troupeau. La physionomie des courbes d'abattage du XVIIIe s. est, par rapport à celle de la période romaine, quasiment inversée.

L'élevage gallo-romain, par le nombre élevé d'animaux sacrifiés en bas âge, ou avant la maturité, et la faible importance des animaux adultes ou vieux, semble favoriser la production bouchère. Les élevages médiévaux traduisent une orientation plus 
diversifiée. La production de viande reste une ressource importante. Elle n'est pourtant pas privilégiée. Les proportions plus élevées d'animaux adultes s'accordent avec l'exploitation de la laine, du lait.

Entre la période gallo-romaine tardive et le XVIIIe s.; la structure d'abattage évolue vers un vieillissement des animaux. Le mode de gestion semble mieux adapté aux besoins d'une rentabilité diversifiée et intensifiée. L'évolution des règles de sélection traduit une profonde modification du rôle économique des petits ruminants (fig. 110 et 111).

Dans les troupeaux ovins de la période galloromaine, l'examen de la forme des chevilles osseuses nous conduit à distinguer plusieurs types d'animaux. Les chevilles osseuses de forme circulaire plus ou moins développées correspondent aux béliers ou aux brebis. L'existence d'animaux châtrés se déduit de la présence de chevilles de conformation plus allongée, dont la face interne est fortement incurvée. Cette interprétation reste à vérifier et à confronter à des observations plus nombreuses. Les proportions entre les trois "sexes" sont assez déséquilibrées. On dénombre dans l'ensemble des échantillons gallo-romains, $52 \%$ de béliers, $22 \%$ de brebis et $25 \%$ d'animaux probablement chatrés. L'importance des mâles semble assez surprenante. Elle trouve un équivalent dans le cheptel caprin. Les boucs y représentent plus de $60 \%$ du troupeau. Les motivations d'une place aussi importante réservée aux boucs et aux béliers nous échappent en grande partie. Il est fort probable qu'un grand nombre ait été réservé à la production bouchère. Dans ce cas l'abattage des agneaux et des cabris les concernerait de manière préférentielle. La production de viande se déduirait aussi de la pratique de la castration dont l'existence et les effets sont peu signalés dans les travaux d'archéozoologie. Elle assure probablement un rendement boucher plus élevé et permet de garder les animaux plus longtemps au sein des troupeaux. Une telle composition réserve une place importante aux animaux de boucherie. La production de la laine peut constituer une ressource complémentaire, celle du lait paraît alors tout à fait secondaire.

$\mathrm{Au}$ cours de la période médiévale, les proportions de béliers et de boucs restent largement majoritaires. La pratique de la castration semble abandonnée.

\section{LE PORC}

La gestion de cet animal apparaît assez uniforme. Les échantillons gallo-romains livrent des courbes qui, à quelques points de détail près (fig. 112), résultent d'un même mode d'exploitation. Celui-ci se résume en un abattage massif des animaux avant l'âge de trois ans. La phase de prélèvement la plus intense se situe entre 0 et 6 mois, ou entre 12 et 24 mois. Quelques animaux, peu nombreux, atteignent et dépassent le stade de la maturité.

Les courbes datées du IXe s. (fig. 113) présentent aussi une physionomie très uniforme. Elles sont marquées par deux pics; l'un situé vers 6 mois, l'autre vers deux ans. Dans tous ces échantillons, les animaux sont éliminés avant leur maturité. Elles diffèrent de celles de la période précédente par la régularité et l'aspect quasi standardisé des phases d'abattage les plus importantes.

Sur les diagrammes d'abattage datés plus tardivement (XVIIIe s.); la boucherie semble concerner de façon préférentielle les porcelets de moins de six mois. Cette classe d'âge supporte les prélèvements les plus intenses. Les animaux sont abattus régulièrement et aucun ne dépasse l'âge de trois ans et demi. Cette gestion surtout basée sur l'abattage 
des animaux en bas âge, vise essentiellement la production bouchère. Cette espèce, du fait de sa prolificité naturelle, y paraît particulièrement bien adaptée.

La sélection des animaux semble soumise à des critères et des règles dont l'application systématique paraît régir de façon très stricte les courbes de la première partie du Moyen Age. Dès le IXe s., l'élevage porcin semble enregistrer les effets de l'intensification de l'utilisation des animaux.

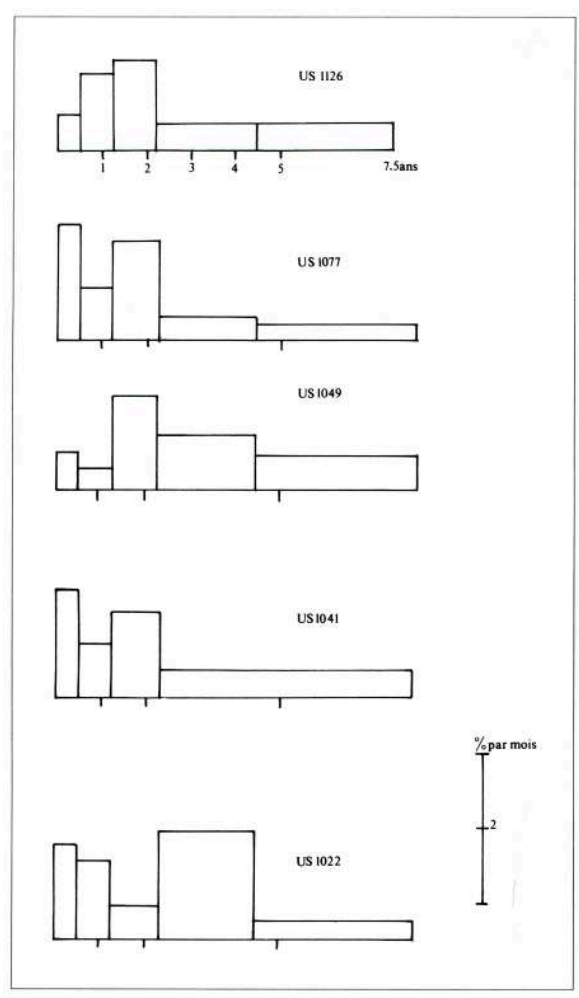

112 - Courbes d'abattage des porcs établies d'après le degré d'épiphysation des os longs. Période gallo-romaine

210 L'estimation du sex-radio au sein des troupeaux est basée sur l'observation des canines inférieures. Celles des verrats sont plus développées que celles des truies. Elles sont plus longues et les racines à croissance continue sont ouvertes. Celles des truies, de taille plus réduite, présentent chez les animaux adultes une racine fermée. Dans les échantillons gallo-romains, les proportions des truies sont légèrement inférieures à celle des verrats. Dans les structures médiévales, elles sont fortement déséquilibrées au profit des mâles qui peuvent représenter jusqu'à $70 \%$ des animaux. Cette importance des verrats est à mettre en rapport avec l'élimination massive qui intervient entre six et trente mois. Le nombre de truies est assez faible. Les critères de choix des animaux en fonction de l'âge et du sexe correspondent à une utilisation exclusivement bouchère. Dès la période gallo-romaine l'exploitation de ces animaux est basée sur des principes dont la pérennité est assurée jusqu'aux temps modernes. 


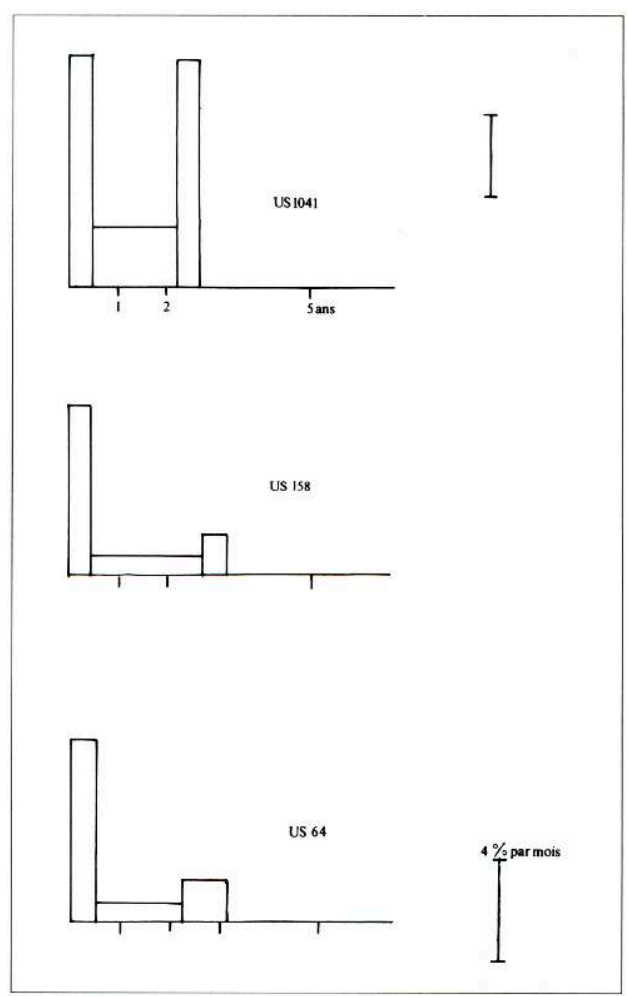

113 - Courbes d'abattage des porcs établies d'après le degré d'épiphysation des os longs. Période médiévale

\section{ÉLÉMENTS DE DESCRIPTION DES ANIMAUX}

\section{LE BCEUF}

Dans les lots d'ossements livrés par les dépotoirs gallo-romains, le bœuf est en général bien représenté. Dans ces échantillons, seuls les métapodes et les phalanges sont restés intacts de toute fragmentation, les métatarses et les métacarpes, particulièrement nombreux, ont livré d'importantes séries de mensurations. Les diagrammes de dispersion, qui permettent d'étudier conjointement deux dimensions, sont structurés en deux, voire trois ensembles de points. La prise en compte de la gracilité et de la longueur totale des os permet de préciser cette dispersion. Ainsi, trois groupes de points distincts sont discernables sur le diagramme correspondant aux métacarpes. Les points situés à la base du diagramme correspondent aux os des vaches. Les plus nombreux, groupés dans la partie supérieure à droite du diagramme, sont à attribuer aux animaux castrés dont les os très graciles sont aussi les plus longs. Le troisième ensemble, celui des taureaux ne compte qu'un seul individu. Cette clé de distinction des os en fonction du sexe des animaux dont ils proviennent s'avère moins efficace dans le cas du métatarse. Le dimorphisme sexuel, semble être la principale mais non la seule cause de variation. La présence d'animaux de types différents est probablement à l'origine de l'importante dispersion des mesures des métatarses.

212 La taille des animaux est une des principales caractéristiques publiées. Cette donnée présente un intérêt certain. Son utilisation est, toutefois, à assortir d'une grande prudence. Toutes les estimations sont dans notre cas effectuées à partir des métapodes dont la longueur, proportionnellement aux autres os des membres, est plus ou moins 
importante. Les hauteurs au garrot, estimées à l'aide des coefficients de Matolcsi (1970), varient entre $1.16 \mathrm{~m}$ et $1.42 \mathrm{~m}$. La moyenne est de $1.24 \mathrm{~m}$ sur la base des métacarpes et de $1.28 \mathrm{~m}$ sur la base des métatarses. Si l'on tient compte de la distribution sexuelle, des fluctuations plus importantes sont observées. La hauteur au garrot moyenne des vaches est de $1.21 \mathrm{~m}$. Elle varie entre 1.17 et $1.26 \mathrm{~m}$. Celle des bœufs est de $1.33:$ le domaine de variation est compris entre $1.27 \mathrm{~m}$ et $1.39 \mathrm{~m}$. La taille du taureau est légèrement inférieure à la taille moyenne des vaches. L'accroissement de la taille des bœufs résulte probablement de la castration. La différence de stature entre les vaches et le taureau est minime. Le faible écart est probablement dû à un dimorphisme sexuel peu accentué. En l'absence d'une série plus importante de métapodes de taureaux, il n'est pas possible de préciser cette observation.

Les hauteurs au garrot des animaux de la période gallo-romaine tardive s'inscrivent dans les domaines de variations enregistrés sur d'autres sites datés de la même période. Elle est en général marquée par un net accroissement de la taille par rapport aux périodes pré-romaines. L'amélioration des races en vue d'une rentabilité bouchère durant la période romaine est probablement la cause principale de cette évolution. La pratique et la maitrise de la castration l'illustrent parfaitement.

214 L'estimation du poids des animaux est un autre élément de description des animaux. Elle nous livre une information relative sur le poids vif des bêtes qu'il ne faut pas confondre avec le poids de viande. Les poids estimés sur la base des métacarpes (coefficients de Matolcsi, 1970) varient entre 550 et $813 \mathrm{~kg}$ dans le cas des taureaux castrés. Le poids des vaches oscille entre 240 et $300 \mathrm{~kg}$. La moyenne est de $280 \mathrm{~kg}$. Les importantes variations au sein de la population de bœufs et entre les deux groupes d'animaux indiquent d'une part la grande variabilité individuelle, et d'autre part l'influence déterminante de la castration sur l'accroissement du poids.

Dans les lots d'ossements médiévaux les os entiers sont rares. Les parties d'ordinaire bien conservées, comme les métapodes, ont été intensément fragmentées. Nous disposons pour cette période de données très dispersées insuffisantes pour documenter l'évolution morphologique des animaux après la fin de l'Empire Romain.

D'après les données, la hauteur au garrot moyenne est $1.25 \mathrm{~m}$ au IXe s., un siècle plus tard elle est de $1.31 \mathrm{~m}$, et de $1.12 \mathrm{~m}$ au XVIIe s. La décroissance de la taille des animaux au cours des temps historiques est un phénomène largement attesté dans toute l'Europe, ainsi que dans le Nord de la France.

\begin{tabular}{|c|c|c|c|}
\hline Période & moyenne & $\mathrm{n}$ & étendue \\
\hline \multirow[t]{3}{*}{ gallo-romain } & vache $\quad 1.21$ & 4 & $1.17-1.26 \mathrm{~m}$ \\
\hline & taureaux 1.19 & 1 & - \\
\hline & castrat 1.33 & 13 & $1.27-1.39 \mathrm{~m}$ \\
\hline IXe & 1.23 & 5 & $1.14-1.31 \mathrm{~m}$ \\
\hline $\mathrm{Xe}$ & 1.27 & 3 & $1.20-1.32 \mathrm{~m}$ \\
\hline XVIIe & 1.09 & 1 & \\
\hline XVIIIe & 1.09 & 1 & \\
\hline
\end{tabular}

Tableau 1 : Données sur les hauteurs au garrot des bovins (coefficients de Matolcsi 1970) 


\section{LES OVICAPRIDÉS}

217 Dans les structures gallo-romaines, les restes osseux bien conservés les plus fréquents sont les chevilles osseuses et les métacarpes.

218 La détermination spécifique et sexuelle des chevilles osseuses, assez aisée, permet de préciser la composition des échantillons d'ossements d'ovicapridés (voir ci-dessus). La biométrie de cette partie du squelette est pourtant très limitée pour plusieurs raisons. La plupart des chevilles osseuses proviennent de sujets jeunes, et ont été systématiquement séparées du crâne à coups de tranchet sur leur base. Les données biométriques qu'elles livrent sont de ce fait incomplètes et ne peuvent être retenues pour l'étude des variations individuelles et des effets du dimorphisme sexuel.

219 Le diagramme de dispersion est caractérisé par une certaine confusion. Il n'est pas structuré en nuages de points bien différenciés. Les points correspondant aux ovins et aux caprins sont organisés en deux populations dont les limites se chevauchent légèrement.

220 D'autres parties du squelette, comme les métapodes, présentent des critères de diagnose sexuelle et spécifique. Les métacarpes et les métatarses entiers sont présents en nombre trop faible pour documenter une étude biométrique.

221 Certaines données, comme la longueur totale des métapodes en particulier, sont à la base des estimations de hauteur du garrot. Etablies à l'aide des coefficients de Teichert, elles varient, pour les métacarpes entre 53 et $66.9 \mathrm{~cm}$, pour les métatarses entre $56.5 \mathrm{~cm}$ et $63.7 \mathrm{~cm}$. Les moyennes respectives sont de $58.3 \mathrm{~cm}$ et $59.9 \mathrm{~cm}$. Il s'agit d'animaux de taille assez importante comparables à ceux d'autres sites du nord et du sud de la France, (Vadet 1981, Jourdan 1976).

222 Les unités statigraphiques datées du Moyen Age et de la période Moderne ont livré des restes osseux d'ovicapridés en quantités plus importantes. Les ossements entiers, peu nombreux, y sont plus fréquents que dans les échantillons de la période précédente.

Les diagrammes de dispersion des mesures des chevilles osseuses sont caractérisés par un étalement et une superposition des nuages de points.

224 La présence de moutons et de chèvres des deux sexes est en partie la cause de cette importante dispersion. Il faut y ajouter les effets de la variabilité diachronique des animaux. En effet les données biométriques dont nous disposons sont dispersées sur plusieurs siècles. L'évolution morphologique des animaux, telle qu'elle a été mise en évidence sur d'autres sites, est caractérisée par une modification importante des proportions du squelette.

225 Les hauteurs au garrot estimées à partir de la longueur totale de plusieurs os (métacarpe, métatarse et radius) sont au cours du Moyen Age légèrement inférieures à celle observées au cours de la période gallo-romaine. Des données plus nombreuses et réparties régulièrement sur tout le champ chronologique concerné restant à réunir pour préciser et compléter cette tendance évolutive. 


\begin{tabular}{|c|c|c|c|}
\hline Période & moyenne & $\mathrm{n}$ & étendue \\
\hline gallo-romain & $59.9 \mathrm{~cm}$ & 14 & $53.0-66.9 \mathrm{~cm}$ \\
\hline $\mathrm{IXe}$ & $56.7 \mathrm{~cm}$ & 10 & $51.8-63.1 \mathrm{~cm}$ \\
\hline $\mathrm{Xe}$ & $58.4 \mathrm{~cm}$ & 4 & $54.1-61.6 \mathrm{~cm}$ \\
\hline XIe & $61.1 \mathrm{~cm}$ & 1 & \\
\hline XVIIe & $57.9 \mathrm{~cm}$ & 6 & $53.1-63.9 \mathrm{~cm}$ \\
\hline
\end{tabular}

Tableau 2 : Données sur la hauteur au garrot des ovicapridés (coefficients de Schramm, 1967)

\section{LE PORC}

D'après les vestiges osseux recueillis dans le contexte gallo-romain, la biométrie de cette espèce ne peut être que très limitée. Les mesures des os longs, constituent des séries réduites. Les proportions entre animaux de l'un et l'autre sexe au sein des échantillons ont fait l'objet du chapitre précédent. Elles ont été établies sur la base des critères distinctifs de la canine inférieure. L'étude de la variation sexuelle, et de ses effets sur la dispersion des mesures des os longs, ne peut être menée faute de données. Il en va de même pour les échantillons d'ossements de porcs datés des périodes médiévale et moderne. Dans ces derniers niveaux, les observations peu nombreuses sont dispersées sur un champ chronologique assez étendu. La hauteur au garrot a été estimée d'après la longueur totale de métapodes (coefficient de Teichert, 1969) seules parties conservées entières dans la plupart des échantillons. Les estimations établies sur cette base présentent, par rapport aux autres os longs, d'importants décalages qui limitent la portée de l'étude biométrique.

Entre le Ve et le Xe s. la hauteur au garrot des porcs est d'environ $77 \mathrm{~cm}$. Au cours des XIe et XIIe s. elle baisse d'environ $3 \mathrm{~cm}$. La taille enregistrée au cours du XVIIe s. est la plus faible. Ce n'est qu'au XVIIIe s. que cette évolution semble s'inverser. La taille augmente très nettement et atteint celle des animaux gallo-romains.

\begin{tabular}{|c|c|c|c|}
\hline Période & moyenne & $\mathrm{n}$ & étendue \\
\hline gallo-romain & $77.2 \mathrm{~cm}$ & 13 & $72.2-83.1 \mathrm{~cm}$ \\
\hline $\mathrm{IXe}$ & $77.7 \mathrm{~cm}$ & 13 & $72.8-84.0 \mathrm{~cm}$ \\
\hline $\mathrm{Xe}$ & $77.3 \mathrm{~cm}$ & 12 & $65.4-84.6 \mathrm{~cm}$ \\
\hline XIe & $76.9 \mathrm{~cm}$ & 2 & $73.4-80.5 \mathrm{~cm}$ \\
\hline XIIe & $74.5 \mathrm{~cm}$ & 2 & $70.7-78.2 \mathrm{~cm}$ \\
\hline XVIIe & $73.7 \mathrm{~cm}$ & 1 & \\
\hline XVIIIe & $77.6 \mathrm{~cm}$ & 1 & \\
\hline
\end{tabular}

Tableau 3 : Données sur les hauteurs au garrot des porcs (coefficients de Teichert, 1969) 


\section{LE CHEVAL}

Les restes osseux d'équidés figurent en proportions très faibles dans les différents échantillons. Ils ont dans la plupart des cas subi la fragmentation bouchère. Les os entiers attribués à cette catégorie d'animaux sont rares. Leurs proportions sont caractérisées par une variabilité dont l'importance relève probablement de la présence de différentes formes d'équidés. Les estimations des hauteurs au garrot varient entre $0,97 \mathrm{~m}$ et $1,46 \mathrm{~m}$. Cette importante dispersion est due à l'hétérogénéité de la population. En effet, les grands chevaux côtoient dès la période gallo-romaine des chevaux de taille moyenne. C'est cependant pendant la période médiévale que cette variabilité est la plus importante. Elle résulte alors probablement de la présence, au côté des différents types d'équidés (chevaux, poneys), d'ânes et de formes hybrides (mulets, bardots), ou d'autres causes comme l'importation d'animaux de grande taille ou la pratique de la castration.

\begin{tabular}{|c|c|c|c|}
\hline Période & moyenne & $\mathrm{n}$ & étendue \\
\hline gallo-romain & $1.12 \mathrm{~m}$ & 1 & \\
\hline Ht Moyen-Age & $1.07 \mathrm{~m}$ & 3 & $0.97-1.19 \mathrm{~m}$ \\
\hline Moyen-Age & $1.27 \mathrm{~m}$ & 2 & $1.08-1.42 \mathrm{~m}$ \\
\hline
\end{tabular}

Tableau 4 : Données sur les hauteurs au garrot des équidés (coefficients de Kiesewalter 1888)

La faiblesse des échantillons est un handicap sérieux pour l'étude de ces animaux dont la détermination ostéologique est très délicate. L'étude de la taille moyenne révèle avant tout la présence ou l'absence de telle ou telle forme, mais ne permet pas de mettre en évidence une éventuelle évolution de la hauteur au garrot.

\section{LE CHIEN}

Dans les structures médiévales la présence de parties complètes de chiens est une source inespérée pour l'étude biométrique de cet animal.

Un seul crâne entier a été recueilli. Ses caractéristiques ne suffisent pas pour proposer une description morphologique de cette espèce.

D'après les os longs, elle est caractérisée par une importante dispersion due à la présence de plusieurs types de chiens. Certains os présentent une torsion assez nette. Cette déformation est le plus souvent caractéristique de chiens du type basset. Les caractéristiques des os longs (longueur, gracilité) ne correspondent pas à un standard précis. L'éventail des tailles estimées (Koudelka 1885) est très large. 


\begin{tabular}{|c|c|c|c|}
\hline & moyenne & $\mathrm{n}$ & variation \\
\hline Humérus & $41-55 \mathrm{~cm}$ & 4 & $29.3-56.9 \mathrm{~cm}$ \\
\hline Radius & $38.3 \mathrm{~cm}$ & 4 & $26.2-54.7 \mathrm{~cm}$ \\
\hline Fémur & $35.5 \mathrm{~cm}$ & 3 & $31.0-44.6 \mathrm{~cm}$ \\
\hline Tibia & $31.7 \mathrm{~cm}$ & 4 & $25.9-43.1 \mathrm{~cm}$ \\
\hline
\end{tabular}

Tableau 5 : Données sur les variations de la hauteur au garrot des chiens (coefficients de Koudelka, 1885)

\section{CONCLUSION}

Les échantillons d'ossements animaux d'origine détritique sont organisés en véritable dépôts de boucherie durant la période gallo-romaine tardive, ou en rejets de moindre importance et moins spécialisés durant le Moyen Age.

Les structures médiévales et modernes ont livré des séries de restes osseux de taille généralement réduite, dont la répartition chronologique est discontinue. L'état fragmentaire ainsi que les lacunes, constituent, aux différents niveaux de l'étude, des handicaps sérieux. Ces remarques s'appliquent dans une moindre mesure aux grands dépotoirs de la période gallo-romaine. Dans ce cas, d'autres facteurs, en particulier la sélection très poussée des vestiges, contribuent à limiter le champ de l'analyse.

\begin{tabular}{|c|c|c|c|c|c|c|c|c|c|c|c|}
\hline \multicolumn{8}{|c|}{ Tableau de décompte des espèces en fonction du nombre et du poids des restes } & \multicolumn{4}{|c|}{ Moderne } \\
\hline & Boeuf & Capridés & Porc & Chat & Chien & Equidés & Sauvages & Divers & oiseaux & Ind. & Total \\
\hline N R & 413 & 518 & 285 & 1 & & 6 & 1 & 10 & 6 & 437 & 1688 \\
\hline P R, en $g$ & 13265 & 4490 & 3185 & & & 85 & & 10 & 115 & 1615 & 22680 \\
\hline \multicolumn{8}{|c|}{ Tableau de décompte des espèces en fonction du nombre et du poids des restes } & \multicolumn{4}{|c|}{ Moyen-Age } \\
\hline & Boeuf & Capridés & Porc & Chat & Chien & Equidés & Sauvages & Divers & oiseaux & Ind. & Total \\
\hline N R & 1899 & 2070 & 2146 & 64 & 111 & 39 & 8 & 65 & 309 & 4056 & 10767 \\
\hline P R, en $g$ & 59820 & 17415 & 23585 & 260 & 660 & 2445 & 315 & 165 & 445 & 15885 & 120995 \\
\hline \multicolumn{8}{|c|}{ Tableau de décompte des espèces en fonction du nombre et du poids des restes } & \multicolumn{4}{|c|}{ Haut Moyen-Age } \\
\hline & Boeuf & Capridés & Porc & Chat & Chien & Equidés & Sauvages & Divers & oiseaux & Ind. & Total \\
\hline N R & 2781 & 2069 & 2372 & 41 & 5 & 55 & 12 & 60 & 386 & 4527 & 12308 \\
\hline P R, en $g$ & 87580 & 20780 & 28540 & 50 & 60 & 3885 & 480 & 1300 & 360 & 33010 & 176045 \\
\hline \multicolumn{8}{|c|}{ Tableau de décompte des espèces en fonction du nombre et du poids des restes } & \multicolumn{4}{|c|}{ Gallo Romain Tardif } \\
\hline & Boeuf & Capridés & Porc & Chat & Chien & Equidés & Sauvages & Divers & oiseaux & Ind. & Total \\
\hline N R & 5857 & 1341 & 1497 & 10 & 43 & 20 & 27 & 20 & 349 & 8073 & 17327 \\
\hline P R, en $g$ & 212735 & 16425 & 19145 & 15 & 515 & 1425 & 995 & & 490 & 77735 & 329480 \\
\hline
\end{tabular}

114 - Tableau de décompte des espèces en fonction du nombre des restes (NR) et du poid des restes (PR, en g.)

Les échantillons sont essentiellement composés d'ossements d'animaux domestiques, les restes d'oiseaux sont rares.

Ceux d'espèces sauvages le sont encore plus. Des conditions de gisement défavorables sont probablement à l'origine de la discrétion de la faune aviaire. Celle des espèces chassées relève probablement d'autres causes: éloignement des campagnes, statut social des quartiers?...

Les variations des proportions relatives des restes des principales espèces consommées traduisent une évolution de l'alimentation carnée des Lyonnais (fig. 114). Animal de 
boucherie par excellence au cours de la période gallo-romaine tardive, le bœuf est durant le Moyen Age concurrencé par le porc et ce dès le IXe s. A partir du XVIe s., la consommation est caractérisée par la prédominance des petits ruminants.

L'évolution de la découpe bouchère est marquée par le morcellement et l'exploitation de plus en plus intensive des différentes parties des animaux. Elle est accompagnée d'une modification de la technique de partage de la carcasse : la fente sagittale de la colonne vertébrale s'impose au cours du Moyen Age plus ou moins rapidement selon les espèces. Parallèlement, au cours du Moyen Age, les règles de sélection semblent, dans le cas de chaque espèce, se préciser et s'adapter à une utilisation plus diversifiée des animaux. Il s'agit d'un élevage polyvalent qui tire un profit maximum de diverses productions animales. Ces modifications interviennent dès le IXe s., leurs effets s'observent en partie aussi dans les échantillons plus récents du XVIe s. .

Les animaux aussi subissent d'importantes transformations. Après la fin de l'Empire Romain, les quelques jalons sur l'évolution morphologique des animaux semblent enregistrer les effets d'une nette tendance à la diminution de la stature, sensible pour toutes les espèces domestiques.

\section{R. -M. ARBOGAST}

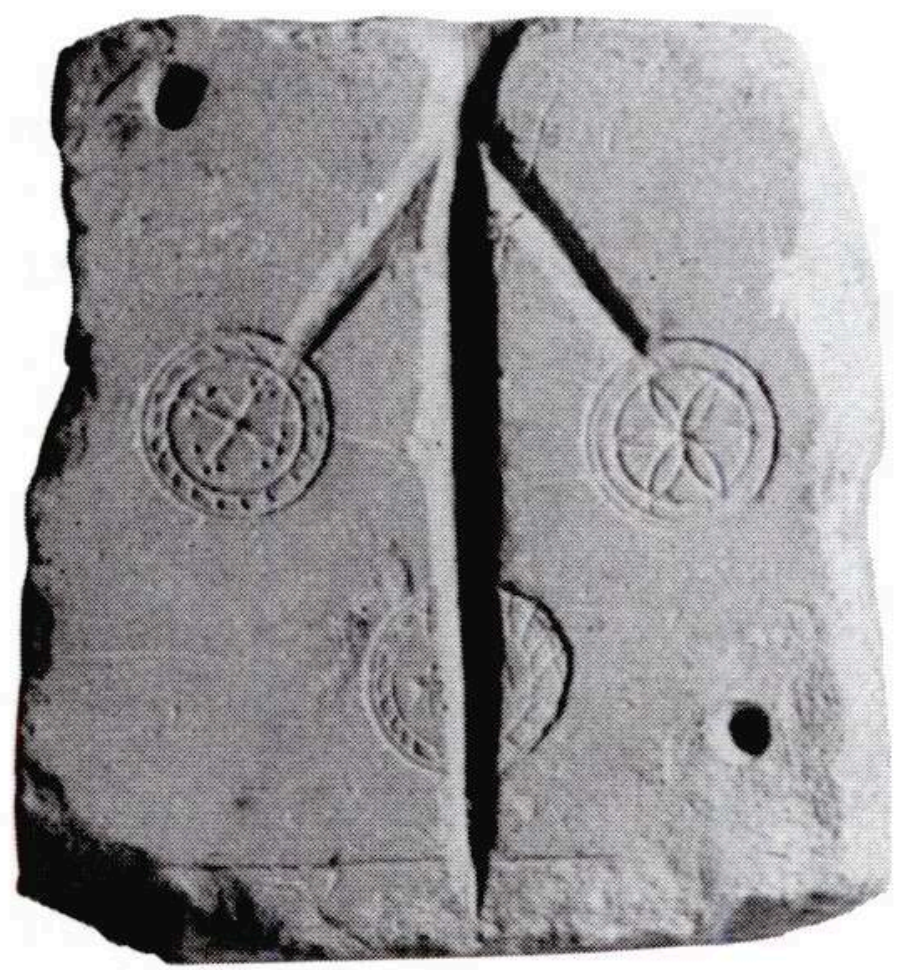

115 - Moule à jetons de jeu. Cf: numismatique médiévale p. 145

\section{MONNAIES ET OBJETS MONÉTAIRES}

\section{NUMISMATIQUE ANTIQUE}

Les chantiers de la rue Tramassac et de la rue Carriès ont livré 107 monnaies antiques :

- 2 du Ier s. ap. J.-C. (1,4 \%) ;

- 2 du IIème s. ap. J.-C. $(1,4 \%)$; 
- 34 du IIIème s. (24,1 \%) ;

- 31 du IVème s. (22 \%);

- 2 du Vème s. (1,4 \%) ;

- 36 monnaies sont illisibles

\section{Tête à droite.}

Autel de Lyon.

$7,78 \mathrm{~g} ; 27 \mathrm{~mm}$

période 20

247 2. Dupondius de Domitien; Rome (85)

IMP CAES DOMITIAN AVG GERM COS XI, tête radiée à droite.

FORTVNAE AVGVSTI [SC], Fortune debout à gauche.

$9,29 \mathrm{~g} ; 27 \mathrm{~mm}$;

RIC 264 var.

période 14

\section{Ile siècle}

248 3. Dupondius de Marc Aurèle ; Rome (161-180)

Tête radiée à droite.

Victoire debout à droite; devant elle, un bouclier.

$9.85 \mathrm{~g} ; 22 / 26 \mathrm{~mm}$

période 3

4. Sesterce de Marc Aurèle ou de Commode ; Rome Tête laurée à droite. 
Illisible.

23,57 $\mathrm{g} ; 31 \mathrm{~mm}$

période 4

250 5. As indéterminé (Ier ou IIe s.)

$7,95 \mathrm{~g} ; 25 \mathrm{~mm}$ (monnaie percée)

période 19

251 6. As indéterminé (Ier ou IIe s.)

$8.86 \mathrm{~g} ; 27 \mathrm{~mm}$

période 14 ?

Ille siècle

252 7. Antoninianus de Gordien III ; Rome (242)

IMP GORDIANVS PIVS FEL AVG, buste radié, cuirassé et drapé à droite, vu de l'arrière.

PM TR P V COS IIPP, l'empereur debout à droite.

$4,00 \mathrm{~g} ; 22 \mathrm{~mm}$

RIC 93

période 3

253 8. Antoninianus de Gallien, règne seul (260-268)

]NVS AVG, tête radiée à droite.

Illisible.

$1,63 \mathrm{~g} ; 17 / 20 \mathrm{~mm}$

période 3

254 9. Antoninianus de Tétricus I ; Atelier I (Trêves, 273)

]RICVS[, buste radié et drapé à droite vu de l'avant.

PAX $[$ AVG]

$1,40 \mathrm{~g} ; 19 \mathrm{~mm}$; flan tordu

Elmer 775

période 2

255 10. Antoninianus de Tétricus I ; Atelier I (Trêves)

]AVG, buste radié et drapé à droite vu de l'avant, légende illisible (spes publica).

$2,17 \mathrm{~g} ; 16 \mathrm{~mm}$

période 11

256 11. Antoninianus de Tétricus II ; Atelier II (Cologne, 273)

C [...] CAES, buste radié et drapé à droite vu de l'arrière.

[PIETA] S AVG [G], Instruments (vase avec anse à gauche). de sacrifice (vase avec anse à gauche).

$1,52 \mathrm{~g} ; 18 \mathrm{~mm}$

Elmer 777 var.

période 4

257 12. Antoninianus de Quintille ; Rome (270)

[IMP CM AVR] CL QVINTILLVS AV [G], buste radié et drapé à droite vu de l'avant.

VICTOR [IA AVG], G à l'exergue.

$2,55 \mathrm{~g} ; 19 \mathrm{~mm}$

RIC 33

période 5 
258 13. Quinaire de Maximien Hercule, Lyon (286)

IMP MAXIMIANVS AVG, buste lauré et cuirassé à droite.

HERC [VLI] AVGG, Hercule debout à droite tenant de la main gauche une massue et la peau de lion et cueillant les pommes des Hespérides sur un arbre autour duquel est enroulé un serpent.

$1,12 \mathrm{~g} ; 15 \mathrm{~mm}$; traces d'argenture

Bastien 48

période 4

259 14. Antoninianus de Dioclétien ; atelier non déterminé (284-294)

[IM] P C VAL DI [OC] LETIANVS PF AVG, buste radié et drapé à droite.

Illisible.

$3,64 \mathrm{~g} ; 21 \mathrm{~mm}$

période 4

260 15-17. Monnaies du IIIe s., non déterminées

$2,27 \mathrm{~g} ; 21 \mathrm{~mm}$; période 14

$3,10 \mathrm{~g} ; 21 \mathrm{~mm}$; période 8

$2,25 \mathrm{~g} ; 22 \mathrm{~mm}$; période 11

Imitations du IIle siècle

\section{a) monnaie coulée}

261 18. Denier coulé d'une impératrice du début du IIIe s.

]AVGUS [TA], buste d'impératrice (Julia Domna ?) à droite.

Illisible.

$2,90 \mathrm{~g} ; 19 \mathrm{~mm}$

période 4

b) Monnaies frappées (le droit porte toujours une tête radiée à droite)

- Imitations au type divo Claudio ; atelier local (post 270)

263 19. Aigle, tête à gauche ; $1,39 \mathrm{~g} ; 14 \mathrm{~mm}$; type RIC 266 ; période 4

20.]NSE[,Aigle, tête à droite ; $1,15 \mathrm{~g} ; 13 \mathrm{~mm}$; type RIC 266 ; période 4

21. Autel ; $2,06 \mathrm{~g} ; 15 \mathrm{~mm}$; type RIC 261 ; période 4

264 - Imitations de Tétricus I et II ; atelier local

265 22.]S PF A / Autel ; 2,00 g ; $19 \mathrm{~mm}$; période 3

23. CAES / HIL-AR[, Hilaritas (revers de Tétricus I) ; $1,50 \mathrm{~g} ; 15 / 16 \mathrm{~mm}$; période 4

24. IMP TE [/ type Hilaritas ; 0,90 g ; $14 \mathrm{~mm}$; période 3

25. Légende informe; type Laetitia ; $1,52 \mathrm{~g} ; 16 \mathrm{~mm}$; période 9

26. Type Pax au sceptre oblique $; 1,63 \mathrm{~g} ; 14 \mathrm{~mm}$; période 14

27.]TE[ / Personnage à gauche $; 2,45 \mathrm{~g} ; 15 / 18 \mathrm{~mm}$; période 278

28 ]TETR[ / légende informe ; 0,35 $\mathrm{g} ; 11 \mathrm{~mm}$; période 4

29.]RIC[ ; Revers illisible ; 0,57 g; $13 \mathrm{~mm}$; période 3

266 - Imitations indéterminées, vraisemblablement des Tétricus

267 30.]S PF AVG/Personnage debout à gauche ; $1,85 \mathrm{~g} ; 15 \mathrm{~mm}$; période 14

31. Revers illisible ; $1,45 \mathrm{~g} ; 19 \mathrm{~mm}$; période 20

32. Revers illisible ; $1,72 \mathrm{~g} ; 15 / 16 \mathrm{~mm}$; période 4 
33. Revers illisible ; $0,93 \mathrm{~g} ; 14 \mathrm{~mm}$; période 11

34. Revers illisible $; 1,20 \mathrm{~g} ; 17 \mathrm{~mm}$; période 13

35. Personnage à gauche $; 1,01 \mathrm{~g} ; 13 \mathrm{~mm}$; période 11

36. Revers illisible $; 1,43 \mathrm{~g} ; 14 \mathrm{~mm}$; période 13

37. Revers illisible ; $0,64 \mathrm{~g} ; 14 \mathrm{~mm}$; période 14

38. Personnage à gauche; $0,57 \mathrm{~g} ; 14 \mathrm{~mm}$; période 16

39. Revers illisible ; $1,27 \mathrm{~g} ; 16 \mathrm{~mm}$ (fragment) ; période 18

40. Revers illisible ; $0,40 \mathrm{~g} ; 13 \mathrm{~mm}$; période 14

Indéterminées (ler-IIle s.)

41-45. Monnaies frustes en bronze (les quatre premières sont probablement des imitations).

269 A. Audra

IVe siècle

270 Toutes les monnaies, des numéros 46 à 91 , sont en bronze

271 46. Atelier de Rome (312-313)

IMP MAXIMINVS PF AVG, buste lauré drapé à droite.

[SPQR]OPTIMO PRINCIPE aigle légionnaire entre deux vexilla.

$3,66 \mathrm{~g} ; 20 \mathrm{~mm}$

$\mathrm{R}-\mathrm{Q}$

RIC $348 b$

période 11

272 47. Atelier de Rome (318-324)

[CRIS]PVS [NOBCAES], buste lauré à droite.

CAE[SARVM NOSTRORVM] VOT X dans une couronne de laurier.

$1,32 \mathrm{~g} ; 18 / 19 \mathrm{~mm}$

$[\mathrm{R}] \mathrm{T}$

RIC 238

période 4

273 48. Atelier indéterminable (319-320)

IMP CONSTAN/[TINVS MAX AVG], buste casqué à gauche.

VICTORIAE[LAET PRINC PERP], deux Victoires portant un bouclier sur lequel il est inscrit

VOT PR

$2,51 \mathrm{~g} ; 18 \mathrm{~mm}$

période 4

274 49. Atelier de Lyon (330-341)

CONSTANTI/NVS [MAXAVG], buste diadémé (rosettes) drapé cuirassé à droite.

GLOR/IAEXERC/[ITVS], deux enseignes entre deux soldats

$1,90 \mathrm{~g} ; 16 \mathrm{~mm}$

$\mathrm{P}(\mathrm{LG})$

RIC 248

période 14 
50. Atelier de Siscia (émission de 330-335)

CONSTANTI/NVS MAX AVG, buste lauré cuirassé à droite.

GLOR/IAEXERC/ITVS, deux enseignes entre deux soldats.

$1,95 \mathrm{~g} ; 16 \mathrm{~mm}$

ASIS

LRBC 742

période 3

276 51. Atelier indéterminable (émission de 335-341) Buste lauré à droite.

Type GLORIA EXERCITVS, une enseigne entre deux soldats.

$0,88 \mathrm{~g} ; 15 \mathrm{~mm}$

période 20

277 52. Atelier de Lyon (341-348)

CONSTANTI/VS PFAVG, buste lauré drapé cuirassé à droite.

VICTORIAE DDAVGGQNN, deux victoires face à face.

$1,68 \mathrm{~g} ; 15 \mathrm{~mm}$

$\mathrm{S}$

Bastien t. XV n 57

période 3

278 53. Atelier oriental (341-348)

DN CONSTAN/[TIVS PF AVG], buste diadémé (perles) à droite.

Type VOT XX MVLT XXX, dans une couronne de laurier.

$0,91 \mathrm{~g} ; 15 \mathrm{~mm}$

période 12

279 54. Atelier indéterminable (341-348)

[DN CO] N S [TAN/S PFAVG], buste lauré, cuirassé en paludamentum à droite.

Type VICTORIAE DDAVGGQNN, deux victoires face à face.

$1,40 \mathrm{~g} ; 15 \mathrm{~mm}$

période 8

280 55. Atelier local. Imitation

Buste à droite.

Deux victoires face à face.

$0,79 \mathrm{~g} ; 11 \mathrm{~mm}$

période 8

281 56. Atelier indéterminable (348-350)

DN CONSTA/NS PFAVG, buste à gauche tenant un globe dans la main gauche.

FE[L. T] EMP./REPAR/ATIO, Virtus sortant un barbare de sa hutte.

$4.10 \mathrm{~g} ; 19 \mathrm{~mm}$

période 10

282 57. Atelier d'Arles (émission de 351-353)

DN MAGNEN/TIVS PFAVG, buste nu à tête droite, A derrière le buste.

VICTORIAE DDNNAVGETCAE, deux victoires tenant un bouclier sur lequel il est inscrit VOT V MVL X.

E

IS

PAR 
$4,45 \mathrm{~g} ; 20 \mathrm{~mm}$

LRBC 347 ; RIC 79

période 7

58. Atelier indéterminable (353-361)

DN CONST[AN]/TIVS PFAVG, buste lauré à droite.

[FEL] TEMP/REPARATIO, l'empereur terrassant un cavalier.

$1,60 \mathrm{~g} ; 17 \mathrm{~mm}$

période 11

59. Atelier indéterminable (353-361)

DN CONST[AN]/TIVS PFAVG, buste diadémé (perles) drapé à droite.

[FEL] TEMP/REPA] RA[TIO], l'empereur terrassant un cavalier.

$1,95 \mathrm{~g} ; 17 \mathrm{~mm}$

période 4

285 60. Atelier indéterminable (353-361)

DN CONSTAN/TIVS PFAVG, buste diadémé (perles) drapé à droite.

[FEL TEMP/]REPARATIO, l'empereur terrassant un cavalier.

$2.11 \mathrm{~g} ; 16 \mathrm{~mm}$

période 8

286 61. Atelier indéterminable (émission de 355-361)

DN CONSTAN/TIVS PFAVG, buste diadémé (perles) drapé à droite.

SPESREI/PVBLICE, Virtus portant un globe et un javelot.

$1,80 \mathrm{~g} ; 15 \mathrm{~mm}$

période 4

287 62. Atelier indéterminable (émission de 355-361)

Buste à droite.

Type SPES REIPVBLICE, virtus portant un globe et un javelot.

$1,30 \mathrm{~g} ; 13 \mathrm{~mm}$

période 11

63. Atelier de Lyon (émission 367-375)

[DN GRATIA]NVS AVGG[AVG], buste diadémé, drapé, cuirassé.

GLORIARO/[MANORVM], l'empereur traînant un captif.

$1,22 \mathrm{~g} ; 16 \mathrm{~mm}$

$\mathrm{I}[\mathrm{S}]$

LVGS

RIC 20 (c)

289 64. Atelier d'Arles (367-375)

DN VALEN/S PFAVG, buste diadémé, drapé, cuirassé.

GLORIA RO/MANORVM, l'empereur traînant un captif.

$1,80 \mathrm{~g} ; 17 \mathrm{~mm}$

$\mathrm{OF} / \mathrm{I}$

CON

RIC 16(b)

période 8

65. [DN VALENTINI]/ANUS PFAVG, buste diadémé drapé cuirassé.

GLORIA RO/[MANORVM], l'empereur traînant un captif.

2,01 g; $15 \mathrm{~mm}$ 
O/FII

CON

RIC 16(a)

période 4

291 66. Atelier de Rome (367 - 375)

DN GRATIAN/VS PFAVG, buste diadémé drapé cuirassé.

SE[CVRITAS/REI]PVBLICAE, victoire à gauche

$2,00 \mathrm{~g} ; 16 \mathrm{~mm}$

R SECUNDA

RIC 24 (c)

période 8

292 67. Atelier de Lyon (émission 375-376)

DN VALENS/PFAVG, buste diadémé drapé cuirassé.

SECVRITAS/[REI] PVBLICAE, victoire à gauche.

$1,99 \mathrm{~g} ; 17 \mathrm{~mm}$

$\mathrm{C} / \mathrm{I}$

$\mathrm{L}$ [VGIP]

RIC 22 (c)

293 68. Atelier indéterminable (364-378)

[DN VALENTINI/]ANUS [PFAVG], buste diadémé, drapé, cuirassé.

Type SECVRITAS REIPVBLICAE, victoire à gauche.

$1,64 \mathrm{~g} ; 16 \mathrm{~mm}$

période 14

294 69. Atelier indéterminable (364-378)

DN VALENTINI/A [NVS PFAVG], buste diadémé, drapé, cuirassé.

[GLO]RI[ARO/MANORVUM], l'empereur traînant un captif.

$0,98 \mathrm{~g} ; 14 \mathrm{~mm}$

période 4

295 70. Atelier indéterminable (364-378)

Buste à droite.

Type SECVRITAS/REIPVBLICAE, victoire à gauche.

$2,00 \mathrm{~g} ; 16 \mathrm{~mm}$

296 71. Atelier indéterminable (364-378)

Buste à droite.

Type SECVRITAS/REIPVBLICAE, victoire à gauche.

$1,78 \mathrm{~g} ; 17 \mathrm{~mm}$

période 15

297 72. Atelier de Lyon (émission de 383-388)

[DN MAG MA] XI/MVS PFAVG, buste diadémé, drapé, cuirassé à droite.

REPARATIO/[REIPVB], l'empereur relevant la république.

$6,20 \mathrm{~g} ; 21 / 22 \mathrm{~mm}$

LVGP

RIC 32 (a)

période 5 


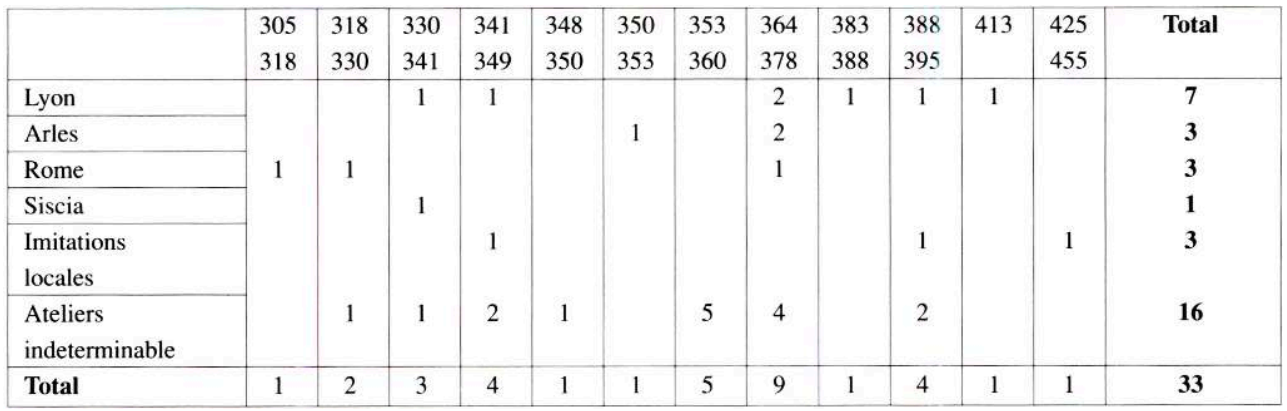

Tableau de répartition par atelier des monnaies des IVe s. et Ve s.

73. Atelier de Lyon (388-395)

[DN ARCA] DIVS [PFAVG]

[VICTOR/IA AV] GGG, victoire à gauche.

$0,88 \mathrm{~g} ; 11 / 12 \mathrm{~mm}$

[L] VGP

RIC 44 (d)

74. Atelier indéterminable (388-395)

Buste diadémé à droite.

Type VICTORIA AVGGG, victoire à gauche.

$0,61 \mathrm{~g} ; 11 \mathrm{~mm}$

période 4

75. Atelier indéterminable (388-395)

Buste diadémé à droite.

Type VICTORIA AVGGG, victoire à gauche.

$0,80 \mathrm{~g} ; 12 \mathrm{~mm}$

période 8

301 76. Imitation de la période 388-395

Buste diadémé à droite.

Type VICTORIA AVGGG, victoire à gauche.

$0,31 \mathrm{~g} ; 8 \mathrm{~mm}$

période 13

Ve siècle

302 77. Atelier de Lyon (émission 411-413)

DN[HONORI/VS PFAVG], buste drapé, cuirassé à droite.

[GLORIA RO/MANO]RVM, l'empereur de face, tenant le labarum, la main gauche sur un bouclier.

Monnaie coupée en deux

$0,79 \mathrm{~g}: 12 \mathrm{~mm}$

[L]VG

LRBC 399

Bastien,t. XVI n ${ }^{\circ} 259$

78. Imitation d'un nummus

émis par Valentinien III (425-455)

Buste diadémé à droite.

Une croix dans une couronne de laurier. 
Type LRBC 867

$0,70 \mathrm{~g} ; 10 / 11 \mathrm{~mm}$

Période 15

79-91 Monnaies frustes en bronze.

\section{Jacquin}

\section{NUMISMATIQUE MEDIEVALE, MODERNE ET CONTEMPORAINE}

Les fouilles archéologiques en milieu urbain procurent habituellement un mobilier abondant et varié. Et l'on pouvait s'attendre à ce qu'une ville comme Lyon n'échappe pas à cette règle, surtout dans un quartier central et densément occupé. Or il semble que les chantiers de Tramassac et Carriès se présentent assez différemment de ce à quoi l'on aurait pu s'attendre. En effet, si la céramique est pour toutes les phases d'occupation, nombreuse et très diversifiée, les autres mobiliers archéologiques sont singulièrement rares. En ce qui concerne les monnaies, leur nombre en premier lieu, étonne par sa modestie : 80 pour une chronologie d'occupation qui va du Ville s. à la fin du XIXe s. Il est vrai que la surface fouillée à Tramassac n'est pas considérable mais si l'on considère que sur ces 80 monnaies, 24 sont contemporaines, on atteint des chiffres singuliers : 45 modernes et 11 médiévales. Il semble que l'on puisse tirer de ces faits deux informations principales.

En premier lieu, il apparaît que c'est l'époque moderne qui est la mieux représentée, depuis la première moitié du XVe s. (12 monnaies de François Ier), jusqu'à la Révolution. Ce quartier a visiblement connu une occupation intense durant toute cette période. Le Moyen Age est comparativement nettement moins présent : 2 monnaies mérovingiennes attestent de l'occupation pendant le Haut Moyen Age, 4 appartiennent à la période XIIe-XIIIe s. et 4 aux XIVe-XVe s. A s'en tenir aux données procurées par cette catégorie d'objets, on en conclurait que le quartier n'a connu qu'à partir du XVIe s., une occupation dense et une activité économique intense.

Les autres données archéologiques nuancent sans doute cette impression.

En second lieu on peut s'interroger sur les raisons de la relative rareté des monnaies. On sait que ces pièces ne peuvent être que le reflet très indirect d'une masse monétaire en circulation, dans la mesure où il ne s'agit évidemment que de pièces perdues. On s'accorde cependant à penser que le fait même que leur présence soit due au hasard les assimile à un échantillonnage, dont la représentativité resterait certes à démontrer, de la pratique réelle. Quoi qu'il en soit, on peut sans doute utiliser ce critère comme confirmation d'un niveau de vie général assez bas de ce quartier, en particulier durant le Moyen Age où il ne constitue pas un lieu d'échanges affirmé. D'utiles indications pourraient être rapportées à ce sujet par la comparaison avec d'autres situations à l'intérieur de l'enceinte canoniale.

Dans ce catalogue, les monnaies ont été classées par époque, et par grandes catégories (royales et seigneuriales). Cette répartition suggère là encore deux réflexions. En ce qui concerne, d'abord, la répartition entre monnayages royaux et seigneuriaux, on constate que la proportion est nettement inversée entre les périodes médiévale et moderne. S'il n'est pas étonnant qu'à l'époque moderne le monnayage royal soit largement prépondérant $(73 \%)$, même dans une région limitrophe avec le Saint Empire, où le volume des échanges avec l'Est peut amener des espèces étrangères, en revanche, pour le Moyen Age, on est frappé de constater l'absence de monnaies royales, 
même pour la fin de la période. Certes des considérations politiques l'expliquent parfaitement, et on ne saurait tirer de conclusions basées sur l'examen de huit monnaies à l'échelle d'une cité comme Lyon. On remarquera cependant que les échanges apparaissent davantage tournés vers l'Est (Savoie, Suisse) que vers le Royaume, ce qui ne correspond qu'imparfaitement à ce que l'on connaît de la géographie de la pratique monétaire à cette époque.

\section{Epoque Médiévale}

310 1.(Inv. $\left.\mathrm{n}^{\circ} 87\right)$

Denier mérovingien de Lyon

(début Ville s.)

(anépigraphe)

Croix cantonnée d'une croisette, d'un point et de deux S.

S/E/D/S/

Croix.

BELFORT n ${ }^{\circ} 2356$ sq.

311 2.(Inv. $\mathrm{n}^{\circ}$ 173)

Denier mérovingien de Lyon ou Vienne

(début Ville s.)

[...]

[...]

BELFORT var.

312 3.(Inv. $\mathrm{n}^{\circ}$ 175)

Denier des Archevêques de Lyon

(XIIe- XIIIe s. ; ap.1157)

$+\mathrm{P}$ [RIMA SE]DES

[Dans le champ : $L, G$ et une barre]

$\mathrm{GA} / \mathrm{L}[\mathrm{L} / \mathrm{I}] \mathrm{A} /[\mathrm{R}] \mathrm{V}$

Croix dont les bras coupent la légende.

argent (Lyon)

POEY D'AVANT n' 5029.

313 4.(Inv. $\mathrm{n}^{\circ}$ 7)

Obole des Archevêques de Lyon

(XIIe- XIIIe s. ; ap.1157)

+ PRIM[A S]EDES

Grand L barré en forme de croix.

+ GALLIARV

Croix pattée.

argent (Lyon)

POEY D'AVANT n ${ }^{\circ} 5039$.

314 5.(Inv. $\left.\mathrm{n}^{\circ} 19\right)$

Denier des Archevêques de Vienne

(XIIe - XIIIe s.)

+ S.M.VIENNA.

Tête de saint-Maurice de profil à gauche.

+ MAXIMA GALL 
Croix pattée cantonnée de quatre besants.

argent (Vienne)

POEY D'AVANT n 4827.

315

6.(Inv. $\left.\mathrm{n}^{\circ} 150\right)$

Denier des Comtes de Blois (XIIe s.)

(anépigraphe)

Tête "dégénérée" avec un besant au-dessus et trois barres au-dessous.

+ BESIS [C]ASTO

Croix pattée.

argent

POEY D’AVANT n 1694.

316 7.(Inv. $\left.n^{\circ} 76\right)$

Denier de Dijon. Fausse monnaie de Hugues IV, duc de Bourgogne (1218-1272).

VGO BVRGVNDIE

Anille coupée par une fasce portant XXVD

DIVIONENSIS

Croix.

argent?

DUMAS n 8-1-3 var.4.

317 8.(Inv. $\left.n^{\circ} 15\right)$

Denier fort réduit d'Amédée IV,

comte de Savoie (1232-1252).

+ :AM :COMES :

Croix pattée.

+ :SABAVDIE :

Etoile.

billon

SIMONETTI, p. $38, n^{\circ} 8 / 1$.

318 9.(Inv. $\left.n^{\circ} 18\right)$

Double denier (1337-1340) de Louis II,

baron de Vaud.

Imitation du double tournois de Philippe VI de Valois.

$+\mathrm{LVD}[\mathrm{DE} \mathrm{SA}]$

Dans le champ : F,R,A,N, deux fleurs de lis et deux annelets.

+ MON[PET CASTRI]

Croix fleurdelisée dont le pied coupe la légende cantonnée de deux annelets.

billon (Pierre-Châtel)

C.N.I. vol.I, pl. XLII, $n^{\circ} 7$.

319 10.(Inv. $\left.n^{\circ} 63\right)$

Obole de blanchet, 1405-07, d'Amédée VIII,

comte de Savoie (1398-1416).

+ [AMEDEVS] CO[MES]

Ecu de Savoie dans un quadrilobe.

$+\mathrm{D}[\mathrm{E} . \mathrm{SABAVD}] \mathrm{IE}$

Croix pleine cantonnée de quatre croisettes.

argent (Chambéry ou Nyons)

SIMONETTI, $\mathrm{N}^{\circ} 26 / 3$. 
11.(Inv. $\left.n^{\circ} 22\right)$

Denier d'Amédée IX, comte de Savoie.

+AMEDEVS : DVX'SABAVDIE :PR

Ecu de Savoie dans un trilobe.

+MARCHIO : IN : ITALIA : PRINCEPS

Croix pattée dans un quadrilobe avec nœuds de l'Annonciade.

Graveur : Michele de Bardonecchia (1468-72)

SIMONETTI, p. $165, n^{\circ} 4 / 2$.

Epoque Moderne

12.(Inv. $\left.n^{\circ} 148\right)$

Denier tournois du Dauphiné, type utilisé sous Louis XII (1498-1515) et François 1er (1515-1547), rois de France.

[...]

Une fleur de lis et un dauphin dans un trilobe aux angles bouletés.

[...]

Croix dans un quadrilobe aux angles bouletés.

billon (Grenoble)

321 13.(Inv. $\left.\mathrm{n}^{\circ} 2\right)$

Denier tournois du Dauphiné de François Ier, roi de France (1515-1547).

[FRANCISCVS.FRANCORV.RX]

une fleur de lis et un dauphin dans un trilobe.

[SIT.NOMEN.DNI.BENEDI]TVM.

Croix dans un quadrilobe cantonné de quatre trèfles.

billon (Grenoble)

DUPLESSY $n^{\circ} 939$.

322 14.(Inv. $\mathrm{n}^{\circ}$ 6)

Denier tournois du Dauphiné, type utilisé sous Louis XII (1498-1515) et François Ier (15151547), rois de France.

[...].FRACO[R] RX.

Une fleur de lis et un dauphin dans un trilobe.

SIT.NOMEN.[DNI].BENEDITVM

Croix dans un quadrilobe cantonné de quatre trèfles.

billon (Grenoble)

323 15.(Inv. $\left.n^{\circ} 8\right)$

Denier tournois, 1515-40, de François Ier, roi de France (1515-1547)

+[F]RAN[CISC]VS.FRANCORV.REX

Deux fleurs de lis dans un trilobe.

+ TV[R]ONVS [CIVIS.FRAN CORV]

Croix pattée dans un quadrilobe.

argent

LAFAURIE $n^{\circ} 727$.

324 16.(Inv. $\left.\mathrm{n}^{\circ} 11\right)$

Double tournois du Dauphiné, 1540-47, de François Ier, roi de France (1515-1547).

+ F[RAN]C[IS]CVS.FR[ANCORV]

Une fleur de lis et un dauphin dans un trilobe. 
[TVRONVS.CIVIS.FRANC.]

Croisette dans un quadrilobe cantonné de trèfles.

billon (Grenoble)

FAFAURIE $n^{\circ} 786$.

17.(Inv. $\left.\mathrm{n}^{\circ} 12\right)$

Liard du Dauphiné, 1515-17, de François Ier, roi de France (1515-1547).

\&FRANCISCVS : DEI :GRA

Grand dauphin à gauche dans le champ.

\&SIT.NOME.DOM

Croix pattée cantonnée de deux couronelles et de deux fleurs de lis.

billon (Grenoble)

FAFAURIE $n^{\circ} 729$.

326 18.(Inv. $\left.n^{\circ} 13\right)$

Double tournois du Dauphiné, type utilisé sous Fouis XII (1498-1515) et François Ier (15151547), rois de France.

[...FRANCOR.REX]

Un dauphin et une fleur de lis dans trilobe.

[SIT.]NOM[EN.DNI.BENEDITVM]

Croix pattée dans un quadrilobe bouleté.

billon (Grenoble).

327 19.(Inv. $\left.\mathrm{n}^{\circ} 16\right)$

Denier tournois du Dauphiné, 1541, de François Ier, roi de France (1515-1547).

+FRAN.D.G.FR[ANCO].R.T.

Deux fleurs de lis et un dauphin dans un trilobe.

*S.NOMEN.D.BE[NE]DIC :

Croisette dans un quadrilobe.

billon (Grenoble)

LAFAURIE $n^{\circ} 796$.

328 20.(Inv. $\left.n^{\circ} 17\right)$

Denier tournois, 1540-41, de François Ier, roi de France (1515-1547).

FRANCISCVS FR[ACORV.REX]

Deux fleurs de lis dans un trilobe.

+ TVRO[NV] S.C[IVI] S :FRANCORV

Croix dans un quadrilobe bouleté.

billon

LAFAURIE $n^{\circ} 792$.

21.(Inv. $\left.n^{\circ} 20\right)$

Denier tournois du Dauphiné, 1515-40, de François Ier, roi de France (1515-1547).

+FRAN[CISC]VS [FRACORV.]REX.

Une fleur de lis et un dauphin dans un trilobe.

[+TVRONVS.CIV]IS.FRANC.

Croix dans un quadrilobe.

billon (Grenoble)

LAFAURIE $n^{\circ} 732$.

330 22.(Inv. $\mathrm{n}^{\circ}$ 21)

Liard du Dauphiné, 1543-47, de François Ier, roi de France (1515-1547).

+FRANCISCVS.D :G :FRANCOR.R. 
Dauphin à gauche.

[+SIT :]NOM[EN :DNI :BENEDICT.]

Croix pleine alésée.

billon (Grenoble)

LAFAURIE $n^{\circ} 795$.

331 23.(Inv. $\left.n^{\circ} 132\right)$

Double tournois de François Ier, roi de France (1515-1547).

+[FRANCISCV]S.D.G.F.REX

Trois fleurs de lis.

+SIT.NOM[EN.DNI.BENEDI.G.T.]

Croisette dans un quadrilobe aux angles bouletés.

billon

LAFAURIE $n^{\circ} 791$.

332 24.(Inv. $\mathrm{n}^{\circ}$ 10)

Monnaie, Allemagne (?), 1662.

[...]SOLERA[...]

Armoiries (indéchiffrables) [16]62

$[\ldots]$

cuivre

333 25.(Inv. $\left.n^{\circ} 35\right)$

Pfening, 1796, de Charles-Théodore, électeur palatin et de Bavière (1777-1799).

(anépigraphe)

Ecu de Bavière

Dans le champ : ${ }^{*} * / \mathrm{PFEN} / \mathrm{NING} / 1796$ dans un grénetis.

cuivre

33426 - 28. Doubles tournois (?)

XVIIe-XVIIIe s.

(illisible)

cuivre

335 Henri III, roi de France (1574-1589) :

Liard au dauphin, 1576 (Grenoble), billon (Inv. $n^{\circ} 54$ ).

Liard au Saint-Esprit, 1583, billon (Inv. $n^{\circ} 56$ ).

336 Henri IV, roi de France (1590-1610):

Douzain, 1593, argent (Inv. $\left.n^{\circ} 47\right)$.

337 Louis XIII, roi de France (1610-1643):

Double tournois, 1638 , cuivre (Inv. $\mathrm{n}^{\circ} 9$ ).

Douzain, 1628 , billon (Inv. $n^{\circ} 73$ ).

Louis XIV, roi de France (1643-1715) :

Liard, cuivre (Inv. $\left.n^{\circ} 41 / 163 / 166 / 167\right)$.

Quinze deniers, 1709-1713 (Lyon ou Metz), billon (Inv. n²3).

Double liard, 1656, cuivre (Inv. $n^{\circ} 52$ ).

339 Louis XV, roi de France (1715-1774) :

Sol, 1740 (Strasbourg), billon (Inv. $n^{\circ} 48$ ). 
Sol, cuivre (Inv. $n^{\circ} 1 / 32 / 34 / 40$ ).

12 deniers, 1792 (Lyon), cuivre (Inv. $n^{\circ} 161$ ).

341

Jeton de Nuremberg,

XIVe-XVe s.

XCEOVSEOPADVLAOPDVEOM

Trois couronnes et trois fleurs de lis.

EPOAVMEPOAVM

Globe surmonté d'une croix dans un double trilobe.

Graveur : Hans Krawinckel

MITTEILUNGEN.

30.(Inv. $\left.n^{\circ} 50\right)$

Jeton de Nüremberg, au type du sol de Louis XIV, roi de France (1643-1715).

LVD XIIII.D G.FR.ET.NAV.REX

Buste lauré à droite. 
[...]FEN[...]NG[...]AUFER

Graveur : Wolff Lauffer (1618-1700)

cuivre doré

MITTEILUNGEN, p. 80.

31.(Inv. $\left.n^{\circ} 193\right)$

Jeton, 1608, de Balthazar de Villars, prévôt des Marchands, gouverneur de Lyon (+1627).

Peut-être émis à l'occasion de son mariage ?

+B.DE VILLARS +1608 PRAESES +LVGD.

Ecu à trois molettes, au chef d'un lion passant, surmonté d'un buste d'homme et orné de lambrequins.

+LOISE.DE.LANGE.DAME.DE.LAVAL.

Ecu en losange parti de Villars et de trois croissants avec un chevron chargé d'une coquille.

cuivre

STEYERT, s.v. "Villars", "Langes".

354 32. (Inv. $\mathrm{n}^{\circ}$ 129)

Moule à jetons de jeu en pierre calcaire, fin du Moyen-Age ou début de l'époque moderne (fig. 115, voir p. 134).

Il comporte trois empreintes gravées en creux et disposées en triangle. Trois canaux de section triangulaire, qui partent d'un même point du bord de la pierre, devaient conduire le métal en fusion dans les empreintes. L'empreinte centrale a été en grande partie cancellée. Deux perforations, disposées dans les angles et diagonalement opposées étaient destinées à maintenir l'autre moitié du moule (absente).

-empreinte 1 :

(anépigraphe)

Etoile à six branches, gravée au compas, entourée d'une bordure festonnée ;

-empreinte 2 :

(anépigraphe)

Croix dont les extrémités sont formées de trois points avec bordure de festons ;

-empreinte 3 :

(anépigraphe)

Décor semblable au ${ }^{\circ} 2$.

356 33.(Inv. $\left.n^{\circ} 24\right)$

Poids monétaire de l'écu d'or au soleil (3,49 gr.), à partir de 1483.

(anépigraphe)

Ecu de France couronné et entouré d'un grénetis.

revers lisse ; forme carrée

cuivre?

DIEUDONNE, $\mathrm{n}^{\circ} 22$.

357 34.(Inv. $\left.n^{\circ} 82\right)$

Poids ou plomb de commerce (?)

(illisible ou lisse)

forme circulaire. 


\section{BIBLIOGRAPHIE}

\section{J.-M. Poisson et B. Claret}

A. de Belfort : Description générale des monnaies mérovingiennes, 5 vol, Paris 1892-95.

L. Ciani : Monnaies royales françaises, de Hugues Capet à Louis XVI, Paris, 1926.

Corpus Nummorum Italicorum, Rome, 1910-1943, 20 vol.

A. Dieudonné : Manuel des poids monétaires, Paris ; 1925.

F. Dumas-Dubourg : Le monnayage des ducs de Bourgogne, Louvain-la-Neuve, 1988.

J. Duplessy : Les monnaies françaises royales de Hugues Capet à Louis XVI, Paris,1989.

E. Fournial : "Monnaies de Lyon et de Vienne“, Cahiers d'Histoire, IV-2, 1959.

V. Gadoury : Monnaies royales françaises, Monaco, 1978.

M. Hoffman : Les monnaies royales de France depuis Hugues Capet jusqu'à Louis XVI, Paris, 1951, 2 vol., Mitteilungen der Baverischen Numismatischen Gesellchaft XXXII/XXXIII (1914-15).

J. Poey d'Avant : Monnaies féodales de la France (Paris, 1858-62), Graz, 1961, 3 vol.

F. de Saulcy : Recherches sur les monnaies des ducs héréditaires de Lorraine, Metz, 1851.

L. Simonetti : Monete italiane medievali e moderne. vol.I, casa Savoia, Florence 1967.

A. Steyert : Armorial général du Lyonnais, du Beaujolais., Paris, 1974.

\section{NOTES}

6. A l'heure de la préparation de ce volume, la reprise de l'étude du mobilier est engagée. Nous devrions donc obtenir rapidement des résultats complémentaires.

7. Un vase peut être représenté par un ou plusieurs tessons mais ne constitue pas forcément une forme archéologiquement complète. Il s'agit en fait de "restes de vase".

8. Durant le VIIe s., la variété des techniques et la diversité des formes du vaisselier se restreignent : exclusivité de la cuisson réductrice, prépondérance de la vaisselle de cuisine sur la vaisselle de table, de stockage et de transport, abandon des importations. Cf., Faure-Boucharlat E., Groupe de travail en céramologie médiévale ; Le Haut Moyen-Age (VIIe-VIIIe), région Rhône-Alpes, Études céramologiques, p. 30.

9. Nous renvoyons à une publication récente sur ce matériel : Desbat A. et Picon M., Sigillée claire B et luisante : classification et provenance. Figlina 7, 1986. p. 5 à 18 et Groupe de travail sur les sigillées claires, Céramiques tardives à revêtement argileux des Alpes du nord et de la vallée du Rhône, ibid, p. 19 à 48. Parmi le matériel examiné dans les lots de Tramassac et Carnés, il faut noter une grande variation d'une pièce à l'autre, le vernis pouvant se révéler soit dur, à reflets métalliques et à pâte sèche donc d'excellente qualité, soit mat et peu adhérent, à pâte savonneuse, ces variations témoignant peut-être de différents groupes d'ateliers qui restent à déterminer.

10. Autrement mentionnée "dérivées des sigillées paléochrétiennes", cette céramique semble poser un problème de dénomination. Voir à ce sujet M.T. Cavailles-Llopis; Les céramiques de l'Antiquité tardive à Marseille, Documents d'Archéologie Méridionale, n 9, 1986, note 81, p. 195. La typologie et les particularismes régionaux sont de plus en plus connus, mais l'évolution 
chronologique de cette production céramique reste encore à cerner (Rigoir 1968). Les caractéristiques des diverses productions sont fondées sur les motifs décoratifs. J. Laprat et Y. et J. Rigoir; Les dérivées des sigillées paléochrétiennes décorées du Gers. Actes du Congrès de Toulouse, S.F.E.C.A.G., 1986, p. 111 à 124.

11. A Lyon, les fouilles opérées sur le chantier Ilot Vieille Monnaie - Service archéologique municipal - n'ont pas livré de matériel de ce type dans les niveaux antérieurs au début du IVe s. Pour l'essentiel, la luisante est généralement attestée dans des niveaux situés à partir du IVe s. Ch. Becker, L. Jacquin ; La sigillée du centre de la Gaule dans trois ensembles de la fin du IIIe s. au milieu du IVe s. sur le site de l'Illot Vieille Monnaie à Lyon, Actes du Congrès de Lezoux, S.F.E.C.A.G, 1989, p. 93 à 100.

12. Malgré l'imprécision de l'évolution chronologique de l'estampée grise, on s'accorde à dater l'apparition de ce type de production à la fin du IVe s. ou au début du Ve s. (Bonifay 1983, p. 329). 13. Les fouilles de la Bourse à Marseille n'ont pas révélé de céramique luisante dans la Période 1 datée de la première moitié $\mathrm{du}$ Ve $\mathrm{s}$. Les récentes fouilles publiées des sites de $\Gamma$ Ouest de la France (Lunel-Viel et Marduel) attestent toutefois la présence de cette céramique tout au long du Ve s.: Raynaud C., Stratigraphie de Marduel (Saint Bonnet du Gard), et Le quartier Sud de l'agglomération antique de Lunel-Viel (Hérault), Documents d'Archéologie Méridionale, 1984, 7, p. 111 à 119 et p. 124 à 147.

14. Cet indice est toutefois à considérer avec prudence car il résulte d'une étude faite sur un matériel funéraire. Cf., FaureBoucharlat E., Reynaud J.F.. Les vases funéraires de la nécropole Saint-Laurent à Lyon, Archéologie médiévale, 1986, XVI. Tous les objets recueillis dans une tombe ne sont pas forcément contemporains.

15. Ce type de décor assez particulier est attesté à l'atelier de Gueugnon. Documents d'Archéologie Française, La terre sigillée gallo-romaine, sous la direction de C. Bémont et J.P. Jacob, $n^{\circ} 6$, Les ateliers du Centre de la Gaule, 1986, p. 167.

16. Les lampes africaines ont fait l'objet d'un classement typologique sommaire élaboré par Hayes J.W. dans son ouvrage, Late roman pottery. Cette rapide typologie, qui reste toutefois le travail de référence, a été développée et complétée par C. Pavolini et L. Anselmino dans Enciclopedia dell'Arte Antica, Atlante delle forme ceramiche, Le lucerne, p. 184 et suiv.

17. M. Janet-Vallat, Saint-Georges, Saint-Pierre, Bâtiments et nécropoles, du IVe s. jusqu'à la Révolution Française, Thèse de IIIe cycle, Aix-Marseille I, 1987, couche 441. Des éléments de comparaison pourront être également tirés de la confrontation de contextes contemporains issus des chantiers lyonnais du Groupe Episcopal dirigé par J.F. Reynaud, d'Adolphe Max dirigé par F. Villedieu, mais également des chantiers périphériques tels que celui de Château-Gaillard (Ain) dirigé par G. Vicherd ou celui de Larina (Hières-sur-Amby) dirigé par P. Porte.

18. Sur le diagramme des céramiques fines, l'augmentation visible des sigillées gauloises, au cours du IVe s., correspond au matériel d'un remblai comportant une quantité importante de céramique résiduelle.

19. Lors de l'inventaire, nous avons regroupé sous l'appellation $B /$ Luisante de nombreux tessons dont l'attribution à l'un ou l'autre des types de production paraissait incertaine. Ils ne figurent pas dans les quantifications proposées ici.

20. Il faut préciser que la céramique luisante mise au jour dans les chantiers archéologiques du secteur Saint-Jean ne semble pas provenir des ateliers de Portout situés au nord du lac du Bourget (Savoie) dont les fouilles ont été dirigées par J. Pernon. Lugdunum ne constituait donc pas un marché privilégié pour ces ateliers proches.

21. Repérages de surface réalisés par $\mathrm{T}$. Odiot, Service régional archéologique en région RhôneAlpes (Odiot-Feuillet, à paraître.)

22. Il semble possible d'attribuer ces fragments à la forme Richeborought 527. Voir à ce propos Bogard Ph. et alii, Des amphores cannelées à Cavaillon (Vaucluse) à la fin du Ier s. de notre ère : 
nouveaux éléments pour l'amphore Richeborought 527, Actes du Congrès de Cognac, S.F.E.C.A.G., 1991, p. 311-321.

23. L'évolution de cette petite amphore a été retracée par H.S Robinson dans, Pottery in the roman period. Vol V., Princeton. New Jersey, 1959.

24. Les découvertes successives de nombreux niveaux d'époque tardive sur les chantiers lyonnais, mais aussi sur ceux des régions périphériques, et l'abondance des céramiques exhumées, posent le problème de l'argumentation chronologique d'un mobilier peu connu donc mal daté. Il apparaît donc urgent de compenser l'évident déséquilibre entre des preuves archéologiques immédiates, c'est-à-dire la présence de restes de vases dans des niveaux stratifiés, et de tout système de référence sur ces objets. Préalablement à la constitution d'un corpus typologique rigide, l'harmonisation des méthodes d'inventaire du matériel, par l'adoption des mêmes définitions des types de céramique et des mêmes procédés de comptage des tessons, facilitera la mise en parallèle des résultats issus des différents chantiers dans le but de définir le profil de l'évolution des groupes de production et de constituer des ensembles régionaux. L'enquête ne fait que commencer. 


\section{Bibliographie}

\section{DOCUMENTS}

Catalogue de l'exposition: Eté-Automne 1980: L'architecture civile à Tours des origines à la Renaissance. Mémoires de la Société archéologique de Touraine. Série in-40, X, Tours, 1980.

Catalogue d'exposition : Des Burgondes à Bavard, mille ans de Moyen Age, recherches archéologiques et historiques., Grenoble, Lyon, Genève, 1981,1984, 238 p., 689 fig.

Catalogue d'exposition : Bourgogne médiévale, la mémoire du sol. Dijon, Musée archéologique, 1987.

Catalogue d'exposition: A travers le verre, du Moyen Age à la Renaissance. Musée et monuments départementaux de SeineMaritime, Rouen, 1989.

Catalogue d'exposition : A la fortune du pot. La cuisine et la table à Lyon et à Vienne (Xe-XIXe s.). Lyon, Vienne, Mâcon, 1990-1991.

Centre national d'archéologie urbaine de Tours 1987 : Centre national d'archéologie urbaine de Tours. Enregistrement des données de fouilles urbaines. Tours, 1987, 100 p.

Collectif Grand Louvre 1984 : Collectif Grand Louvre. Fouilles archéologiques, Cour Napoléon, Paris, 1984.

Colloque 1977 : Actes du colloque tenu à Tours du 10 au 14 mai 1977. Recherches sur l'habitat urbain en Europe au XVe et au XVIe s., la maison de ville à la Renaisssance (De Architectura). Ed. Picard, 1983, $200 \mathrm{p}$.

Colloque 1983 : Architecture de terre et de bois. Actes du deuxième congrès archéologique de Gaule Méridonale. Lyon, 2 au 6 novembre 1983. Publié sous la direction de Jacques Lasfargues. (D. A. F.) Maison des Sciences de l'Homme, Paris, 1983,192 p.

Colloque 1980 : Actes du colloque international d'archéologie urbaine, Tours. Ministère de la culture et de la communication. Paris, $1980,769 \mathrm{p}$.

Congrès d'archéologie chrétienne 1986: Congrès d'archéologie chrétienne. Imprimerie Multitude, Lyon, 1986.

Laboratoire d'archéologie urbaine 1985 : Laboratoire d'archéologie urbaine. Recherches sur Tours. Impression, La Simarre, 4, 1985, 55 p.

Rapport de fouilles 1983 : Ville de Saint-Denis. Imprimerie municipale de Saint-Denis. SaintDenis, 1983,200 p., 98 fig. 
Artaud 1846 : ARTAUD (F.) - Lyon souterrain ou observations archéologiques et géologiques faites dans cette ville depuis 1794 jusqu'en 1836. Lyon, 1846, $259 \mathrm{p}$.

Ayala 1992: AYALA (G.) - Avec la coll. de VEROT-BOURRELY (A.) Rue Palais-Grillet: une intervention archéologique au cœur de la Presqu'île Lyonnaise.R.A.E.,t. 43, fasicule 2, 1992, pp. 293-305.

Audin 1957 : AUDIN (A.), MEJAT (R.) - Fouilles Rue Tramassac. Cahiers Rhodaniens IV, 1957, p. 57-61.

Audin 1959 : AUDIN (A.) - Essai sur la topographie de Lugdunum. Institut d'Etudes Rhodaniennes. 2 éd. Lyon, 1959, $179 \mathrm{p}$.

Audin 1910 : AUDIN (M.) - Répertoire des plans et vues générales de la ville de Lyon, des origines à la fin du XVIIe s. Revue d'Histoire de Lyon,. 1910, Lyon, p. 204-229.

Auduin 1987 : AUDUIN (F.) et MARINVAL-VIGNE (M.C.) - Boucherie médiévale et moderne dans le Val-de-Loire, France. Anthopozoologica $n^{\circ}$ Spécial, 1987, Paris, p. 45-52.

Auger 1990 : AUGER (M.) - Lyon, verreries des XVe et XVIIe s. in : Verrerie de l'est de la France, XIIe-XVIIIe s. Fabrication, consommation. R.A.E. 1990, p. 277-293.

Barone 1976 : BARONE (R.) - Anatomie comparée des mammifêres domestiques. Ostéologie, 2 vol. éd. Vigot, Paris, 724 p., 401 pl.

Bastien 1985-1987 : BASTIEN (P.) - Le monnayage de l'atelier de Lyon, Numismatique Romaine, Wetteren, Belgique, t. XV, 1985, t. XVI, 1987.

Bats 1986 : BATS (M.), BEYSSAC (J.-C.), CHAZELLES (C.-A.), FICHES (J.-L.), POUPET (P.), PY (M.) Enregistrer la fouille archéologique, le système élaboré pour le site de Lattes (Hérault). Lattes, 1986.

Becker 1982-1983 : BECKER (Chr), LASCOUX (J.-P.) - Station funiculaire et Place Bellecour. Rapport d'intervention, Service archéologique de la Ville de Lyon, 1982-83.

Becker 1989 : BECKER (Chr.), AUGER (M.), VICARD (T.) - Les fouilles de la place Antonin Poncet à Lyon. Archéologie du Midi médiéval, t. 7, 1989.

Bonifay 1983 : BON1FAY (M.) - Eléments d'évolution des céramiques de l'Antiquité tardive des fouilles de la Bourse à Marseille. R.A.N., 1983, XVI.

Bravard 1983 : BRAVARD (J.-P) - Les sédiments fins des plaines d'inondations dans la vallée du Haut-Rhône. Revue de Géographie Alpine, t. LXXI, 4, p. 163-179.

Bravard 1984 : BRAVARD (J.-P.) - Avec la coll. de C. AMOROS et C. JACQUET (Université Lyon 1). Etude géomorphologique de site. Avenue A. Max (Lyon 5ème). Université J. Moulin, Département de Géographie, rapport dactylographié, 1984.

Bravard 1985: BRAVARD (J.-P.), AMOROS (C.), JACQUET (c.) - Reconstitution de l'environnement des sites archéologiques fluviaux par une méthode interdisciplinaire associant la géomorphologie, la zoologie et l'écologie. Revue d'Archéométrie, Vol. 10, p. 43-45.

Bravard et alii 1989 : BRAVARD (J.-P.), BURNOUF (J.), VEROT (A.) - Géomorphologie et archéologie dans la région lyonnaise: questions et réponses d'un dialogue interdisciplinaire. Bulletin de Société Préhistorique Française, 1989, t. 10/12, p. 429-440.

Brongnart 1877 : BRONGNART (A) - Traité des arts céramiques. Paris. 1877.

Carandini et alii 1968 : CARANDINI (A.) et collab. - Ostia I, le terme del Nuotatore. Studi miscellanei, 13, 1968.

Chapelet 1980 : CHAPELOT (J.), FOSSIER (R.) - Le village et la maison au Moyen Age. Bibliothèque d'Archéologie, Hachette littérature, 1980, 357 p. 
Chedeville 1980 : CHEDEVILLE (A.), LE GOFF (J.), ROSSIAUD (J.) - La ville médiévale des Carolingiens à la Renaissance. Histoire de la France Urbaine, sous la direction de G. DUBY. Ed. du Seuil, 2, Paris, 1980,653 p.

Chevalier 1981 : CHEVALIER (B.) - Le paysage urbain à la fin du Moyen Age : imaginations et réalités. p 7-21. in : Le paysage urbain au Moyen Age. Actes du XIème congrès des historiens médiévistes de l'enseignement supérieur Ed. P.U.L, Lyon, 1981,279 p.

Colardelle 1983 : COLARDELLE (M.) - Sépultures et traditions funéraires du V au XIIIe s. après J.C. in : Les Alpes françaises du Nord. Grenoble, 1983.

Coville 1928 : COVILLE (A.) - Recherches sur l'histoire de Lyon, du Ve au XIe s. (450-820). Ed. A. Picard, Paris, 1928.

Démians d'Archimbaud 1979-1980 : DEMIANS D'ARCHIMBAUD (G.), VALLAURI (L.), THIRIOT (J.). - Céramiques d'Avignon. Les fouilles de l'hôtel de Brion et leur matériel. Mémoires de l'Archéologie du Vaucluse, 7e série, t. 1, 1979-1980.

Démians d'Archimbaud 1980 : DEMIANS D'ARCHIMBAUD (G.) - Les fouilles de Rougiers. CNRS, Paris, 1980, 724 p., 520

Desbat 1978 : DESBAT (A.) - La céramique à vernis noir "métallescente" de la rue des Farges. Bulletin de liaison de la Direction des Antiquités Historiques Rhône-Alpes, n 8, p. 40 à 53.

Desbat et alii 1979 : DESBAT (A.) LAROCHE (C.) MERIGOUX (E.) - Notes préliminaire sur la céramique commune de la rue des Farges. Figlina 4, p. 1-17, 1979.

Desbat 1981 : DESBAT (A.) - Notes sur l'abandon de la ville haute de Lyon. Récentes recherches en archéologie gallo-romaine et paléochrétienne sur Lyon et sa région, volume sous la direction de S. WALKER. B.A.R. International series 108. Lyon, 1981,p. 105-117.

Desbat 1981 : DESBAT (A.) - L'architecture de terre à Lyon à l'époque romaine. ibid. 18, 1981, p. 55-81.

Desbat et alii 1981 : DESBAT (A.) et WALKER (S.) - Le problème des origines de Lyon. Ibid. 1981, p 29-54.

Desbat 1982 : DESBAT (A.) - Lyon et ses fleuves dans l'Antiquité. Catalogue d'exposition Lyon au fil des fleuves. Lyon, 1982.

Desbat 1986: DESBAT (A.) - Les fouilles de la me des Farges. 1974-1980. Groupe Lyonnais de Recherches Archéologiques Gallo-Romaines et Ville de Lyon. Lyon, 1986.

Desbat 1988 : DESBAT (A.) - La sigillée claire B : état de la question, SFECAG, Actes du congrès d'Orange, 1988, p. 91-99.

Desbat à paraître: DESBAT (A.) et MANDY (B.) - Le développement de Lyon à l'époque Augustéenne : l'apport des fouilles récentes. Colloque sur les villes Augustéennes tenu à Autun 1985.

Duby 1980 : DUBY (G.) - Histoire de la France Urbaine. La ville antique. Ed. du Seuil, 1980, 606 p.

Duby 1980 : DUBY (G.) - Histoire de la France Urbaine. La ville médiévale. Ed. du Seuil, 1980, 656 p.

Duchesne 1902 : DUCHESNE (L.) - Origines du culte chrétien. Paris, 1902, 3e éd.

Esquieu 1983 : ESQUIEU (Y.) - Mutations d'un quartier canonial au bas Moyen Age : l'exemple de Viviers. 108ème congrès National des Sociétés Savantes. Grenoble. 1983, Archéologie, 1987, p. 297-311.

Faure-Boucharlat 1980 a : FAURE-BOUCHARLAT (E.) - La céramique médiévale en lyonnais et recherches sur les moyens de production. Thèse de doctorat de 3e cycle, dactylographiée. Université de Lyon 2, 1980. 
Faure-Boucharlat 1980 b : FAURE-BOUCHARLAT (E.) et alii - Eléménts comparatifs de la production céramique du XIe s. dans le bassin rhodanien. Actes du Colloque International : la céramique médiévale en Méditerranée occidentale (Xe-XVe s.). (Valbonne 1978). CNRS, 1980, p.429-440.

Faure-Boucharlat 1981 : FAURE-BOUCHARLAT (E.) - Aire de répartition de la céramique à fond marqué. Des Burgondes à Bayard mille ans de Moyen-Age. Recherches archéologiques et historiques. Catalogue de l'exposition, Grenoble-Lyon-Genève, 1981-1984.

Faure-Boucharlat 1986 : FAURE-BOUCHARLAT (E.) et REYNAUD (J.-F.) - Les vases funéraires de la nécropole SaintLaurent de Lyon. Archéologie Médiévale, t. XVI, 1986, p. 41-64.

Faure-Boucharlat 1993: FAURE-BOUCHARLAT (E.) MACCARI-POISSON (B.) - Le mobilier céramique in: Les habitats du lac de Paladru (Isère) dans leur environnement (sous la direction de COLLARDELLE (M.) et VERDEL (E.). DAF nº 40, 1993, p. 189-203.

Ferdière 1980 : FERDIERE (A.) - La fouille, pour quoi faire? in SCHNAPP (A.) L'archéologie aujourd'hui. Paris, 1980, p. 2360.

Fevrier 1986 : FEVRIER (P.-A.), PICARD (J.-Ch.), PIETRI (Ch.), REYNAUD (J.-F.), 1986, Lyon, p. 15-5. in : Province ecclésiastique de Lyon (Lugdunensis Prima) par BEAUJARD (B.), FEVRIER (P.-A.), PICARD (J.-Ch)., PIETRI (C.), REYNAUD (J.-F.), IV. Topographie chrétienne des cités de la Gaule des origines au milieu du VIIIe s. Editée par N. GAUTHIER et J.-Ch. PICARD. Ed. De BOCCARD, Paris, 1986, 80 p.

Fevrier 1988 : FEVRIER (P.-A.), FIXOT (M.), RIVET (L.) - Au cœur d'une ville épiscopale. Fréjus. Comité d'Animation et d'Actions Culturelles. Fréjus, 1988, 60 p.

Fixot 1980 : FIXOT (M.) - L'habitat Urbain, in: DUBY (G.) Histoire de la France Urbaine. La ville médiévale. Ed. du Seuil, 1980, 656 p.

Fixot 1986 : FIXOT (M.), GUYON (J.), PELLETIER (J.-P.), RIVET (L.) - Des abords du forum au palais archiépiscopale. Etude du centre monumental d'Aix-en-Provence. Bulletin monumental, 144. III, Société Française d'Archéologie, 1986, 290 p.

Galinié 1979 : GALINIE (H.) et RANDOIN (B.) avec HOLTZ (M.) et KEMP (R.-L.) - Les archives du sol à Tours. Survie et avenir de l'archéologie de la ville. Ed. La simarre. Tours, 1979, 63 p.

Gaspard 1973 : GASPARD (P.) - Les bouchers, collection la Magie des Métiers. Editions Neuf, Paris, 1973.

Grataloup 1988 : GRATALOUP (C) - Les céramiques à parois fines de la rue des Farges à Lyon, BAR International Series 457, 1988.

Grant 1987 : GRANT (A.) - The use of tooth wear as a guide to the age of domestic ungulates in ageing and sexing animal bones from archaelogical sites, BAR BS 109 1982, p. 41-64 et 91-109.

Gonthier 1981 : GONTHIER (N.) - Le paysage urbain au Moyen Age, une esquisse du paysage urbain lyonnais au XIVe et XVe s. Ed. P.U.L., Lyon, 1981, 279 p.

Guigue 1867 : GUIGUE (M.-C.) - Obituaire de l'Eglise de Lyon. Lyon, 1867, 323 p.

Hatting 1975 : HATTING (T.) - The influence of castration on sheephorns in Clason A.T.: Archaelogical Studies 345-355, 11 fig.

Hayes 1972 : HAYES (J. H.) - Late roman pottery, London, 1972.

Ijzzereef 1981 : IJZZEREEF (G.F.) - Berichten van Bronze age animal bones from Bovenskarpel. The excavations at het Valkje. De Rijksdienstvoor het Oudheidkundig Bodemonderzoek, $228 \mathrm{p}$. 116 fig. Amersfoort.1981.

Jacquin 1981 : JACQUIN (L.) - 8, Rue de la Quarantaine. Rapport de fouilles. Service archéologique de la Ville de Lyon, 1981.

Jacquin 1984 : JACQUIN (L.) - 1984 à 1987. Ilot 24, 7 et 11 Rue de la Monnaie. Rapports de fouilles, Service archéologique de la Ville de Lyon, 1984. 
Jeanjacquot 1993 : JEANJACQUOT (J.-C.) (sous la direction de) - Pymont, la forteresse oubliée. Centre Jurassien du Patrimoine, Lons-le-Saunier, 1993.

Jourdan 1976 : JOURDAN (L.) - La faune du site galloromain et Paléochrétien de la Bourse à Marseille. CNRS Aix Marseille, 1976.

Keay 1984 : KEAY (S. J,) - Late roman amphorae in the western mediterranean, a typology and economic study : the catalan evidence, BAR International Series 196, 1984.

Kleinclausz 1925 : KLEINCLAUSZ (A.) - Des origines à nos jours. La formation de la cité. Lyon, 1925.

Kleinclausz 1939 : KLEINCLAUSZ (A.) - Histoire de Lyon. Des origines à 1595. Lyon, 1939.

Kiesewalter 1888 : KIESEWALTER - Cité par Van Den Driesch A. et Boessneck J. 1974 p. 333, 1888.

Koudelka 1885 : KOUDELKA (F.) - Cité par Van Den Driesch A. et Boessneck J. 1974 p. 343, 1885.

Lamboglia 1963 : LAMBOGLIA (N.) - Nuove osservazioni sulla "terra sigillata chiara", II. in : RSL, 29, 1963.

Lasfargues 1976 : LASFARGUES (J.), VERTET (H.) - Les estampilles sur sigillée lisse de l'atelier augustéen de la Muette à Lyon. Figlina, 1, 1976, p. 39-87.

Laubenheimer 1985 : LAUBENHEIMER (F.) - La production des amphores en Gaule Narbonnaise, Centre de recherches d'Histoire Ancienne, Annales littéraires de l'Université de Besançon, vol. 66, 1985.

Lavedan 1974 : LAVEDAN (P.), HUGUENEY (J.) - L'urbanisme au Moyen Age. Ed. Presses de Savoie. AmbillyAnnemasse. 1974, 173 p., 577 fig.

Le Masne de Chermont 1987 : LE MASNE DE CHERMONT (N.) - Les fouilles de l'ancien Evêché de Poitiers (Vienne). Revue Aquitania.,V, 1987. p. 149-175.

Leenhardt 1988 : LEENHARDT (M.) et VALLAURI (L.) - Le cimetière du cloître cathédral de Viviers. Le mobilier céramique, in : ESQUIEU (Y.). Viviers, cité épiscopale, D.A.R.A. n. 1, Lyon, 1988, p. 81-100.

Leguay 1984 : LEGUAY (J.-P.) - La rue au Moyen Age. Coll. de Mémoire d'homme. Ouest France, Rennes, 1984, $253 \mathrm{p}$.

Lesne 1910 : LESNE (E.) - La propriété ecclésiastique en France aux époques romaine et mérovingienne. Lille-Paris, 1910.

Louvet 1981 : LOUVET (Y.), PERRODIN (J.) - 13 novembre 1930, la catastrophe de Fourvière. Ed. Renaissance du Vieux Lyon, Lyon, 1981.

Maccari-Poisson 1988: MACCARI-POISSON (B.) - Un dépotoir du bas Moyen-Age dans le quartier Saint-Jean à Lyon. Archéologie Médiévale, t. XVIII, 1988, p. 215-237.

Maccari-Poisson 1992 : MACCARI-POISSON (B.) - Contribution à l'étude des poteries du Haut Moyen-Age: le quartier Saint-Jean à Lyon (IX-Xe s). Actes du IVe Colloque International: Cerâmica medieval do Mediterrâneo ocidental (Lisbonne 1987), Mertola, 1992, p. 147-160.

Macé 1991 : MACE (S.), VEROT-BOURRELY (A.) et BRAVARD (J.-P.) - Genèse et fonctionnement holocène de la plaine alluviale du Rhône à Lyon. 116ème congrès Nat. des Soc. Sav., Chambery, 1991, p. 17-31.

Mandy 1983: MANDY (B.) - Le quartier antique du Verbe Incarné. Histoire et Archéologie, les dossiers, 78, novembre 1983, p. 23-26.

Matolcsi 1970: MATOLCSI (J.) - Domestikations Forschung und Geschichte der Haustiere. Internationales Symposium in Budapest, p. 377-3. Budapest 1971.

Méniel 1984 : MENIEL (P.) - Contribution à l'histoire de l'élevage en Picardie, du Néolithique à la fin de l'Age du Fer. Revue Archéologique de Picardie, numéro spécial, 1984, 56 p. 
Meyer 1985 : MEYER (O.), WYSS (M.) - Saint-Denis, recherches urbaines. 1983 à 1985. Bilan des fouilles. Imprimerie Municipale de Saint-Denis, Saint-Denis, 1985, 111p., 75 fig.

Mounier 1986 : MOUNIER (R.) - Etude historique et archéologique de la Maison du Chamarier du cloître Saint-Jean à Lyon. Mémoire de maîtrise. Université Lyon II, sous la direction de Jean-François REYNAUD. Lyon, 1986, 146 p., 46 ill., dactylographié.

Olive 1987 : OLIVE ( $\mathrm{Cl}$.) - Quelques aspects de la technique de débitage des bovidés en boucherie gallo-romaine dans la vallée du Rhône et les Alpes du Nord. Anthopozoologica, numéro spécial, 1987 p.77-83.

Panella 1972 : PANELLA (C.) - Stratigrafia delle terme ostiensi del Nuotatore. Recherches sur les amphores romaines. Rome, 1972.

Paunier 1981: Paunier (D.) - La céramique gallo-romaine de Genève. Société d'histoire et d'archéologie de Genève, 1981.

Pesez 1984 : PESEZ (J.-M.) (sous la direction de) - Brucato, histoire et archéologie d'un habitat médiéval en Sicile. Coll. de l'école Française de Rome. $n^{\circ}$ 108, Rome, 1984, 2 vol., 827 p.

Pesez 1985 : PESEZ (J.-M.) - La terre et le bois dans la construction médiévale. Architecture de terre et de bois. Actes du deuxième congrès archéologique de Gaule Méridionale. Lyon du 2 au 6 novembre 1983. Publié sous la direction de Jacques LASFARGUES. D. A. F., $n^{\circ}$ 2, Ed. de la Maison des Sciences de l'Homme, Paris, 1985, p. 159-167.

Pesez 1986 : PESEZ (J.-M.) - Le foyer de la maison paysanne (XIe, XVe s.). Archéologie Médiévale, XVI, Ed. du CNRS, 1986, 28 p., 8 fig.

Peiry 1988 : PEIRY (J.-L.) - Approche géographique de la dynamique spatio-temporelle des sédiments d'un cours d'eau intra-montagnard: l'exemple de la plaine alluviale de l'Arve (Hte-Savoie). Doctorat de Géographie et Aménagement, Univ. J. Moulin-Lyon III, 378 p.

Plongeron 1974 : PLONGERON (B.) - La vie quotidienne du clergé français au XVIIIe s., Paris Hachette, 1974, 284 p.

Randoin 1980 : RANDOIN (B.) - Fouilles et enregistrement d'un site urbain. Mémoire de EHESS, Paris, 1980.

Reichstein 1974 : REICHSTEIN (H.) - Untersuchungen an Tierknochenfunde 1943-1964. Berichte iiber die Ausgrabungen in Haithabu. Neumünster, 1974.

Renimel 1974 : RENIMEL (S.). - L'atelier céramique de Sevrey (IXe-XIXe s.). Un millénaire de tradition céramique en Chalonnais. Châlon-sur-Saône, 1974.

Reynaud 1977 : REYNAUD (J.-Fr.), VICHERD (G.), JACQUIN (L.) - Les fouilles retrouvent l'enceinte fortifiée de la ville basse près de la Saône. Archeologia. 112, novembre 1977, p. 50-59.

Reynaud 1978 : REYNAUD (J.-Fr.), VICHERD (G.), JACQUIN (L.) - L'enceinte réduite de Lugdunum, 1978, p. 213-260. Colloque travaux militaires en Gaule Romaine et dans les Provinces nord-ouest. Caesarodunum. Bulletin de l'Institut d'Etudes Latines et du Centre de Recherches. A. Piganiol, 1978.

Reynaud 1979 : REYNAUD (J.-Fr.), JACQUIN (L.), VICHERD (G.) - Enceinte du cloitre de Saint-Jean. Sondage $n^{\circ} 9$ Rue Tramassac. Rapport de fouilles, U.R.A. 26 du CNRS, 1979.

Reynaud 1981 : REYNAUD (J.-Fr.) - Lyon du IVe au Ville s. Edifices religieux, nécropoles et topographie urbaine. ALSSAM. Lyon, 1981,40 p., $19 \mathrm{ftg}$.

Reynaud 1986 : REYNAUD (J.-Fr.) - Les fouilles de Saint Laurent-de-Choulans. Rapport de fouilles, S.R.A., 1985.

Reynaud 1986 : REYNAUD (J.-Fr.) - Lugdunum christianum. Thèse dactylographiée de doctorat d'Etat. Université de Paris IV, 1986.

Reynaud 1986 : REYNAUD (J.-Fr.) - Lyon aux premiers temps chrétiens: basiliques et nécropoles. Guides Archéologiques de la France. Ministère de la Culture et de la Communication, 1986. 
Rigoir 1968 : RIGOIR (J.) - Les sigillées paléochrétiennes grises et orangées, Gallia, 1968, XXVII, 1, p. 177 à 244 .

Rodet-Belarbi et Yvinec 1990 : RODET-BELARBI (I.) et YVINEC (J.H) - Boucheries et dépotoirs de boucherie galloromain, Anthropozoologica, 13, p. 19-26.

Russo et alii 1961 : RUSSO (Ph.), AUDIN (A.) - Le site de Lyon. Panorama de son évolution. Revue de Géographie de Lyon, p. 295-348.

Russo et alii 1964 : RUSSO (Ph.), AUDIN (A.) - Histoire ancienne de Lyon. Lyon, 1964.

Sachet 1914 et 1918 : SACHET (A.) - Le pardon annuel de la Saint-Jean et de la Saint-Pierre à SaintJean de Lyon. 13921790. Saint-Jean du XIVe au XVIIIe s. Lyon, 1914 et 1918, 2 vol.

Schnapp 1974 : SCHNAPP (A.) - L'archéologie. Faire de l'histoire II. Nouvelles approches. Sous la direction de Jacques LE GOFF et de Pierre NORA. Coll. Folio Histoire. Ed. Gallimard, 1974, p. 11-36.

Schnapp 1980 : SCHNAPP (A.) - L'archéologie aujourd'hui. Paris, 1980.

Schramm 1967 : SCHRAMM. - Cité par Van Den Driesch A. et Boessneck J. 1974, p. 341.

Steyert 1895-1899: STEYERT (A.) - Nouvelle Histoire de Lyon et des provinces du Lyonnais, Forez, Beaujolais, Franc-Lyonnais et Dombes. Lyon, 4 vol., 1899.

Teichert 1975: TEICHERT (M.) - Ostéométrische Untersuchungen zur Berechnung der Widerristhöhe bei Schafen. in Clason A.T. Archaezoological Studies, p. 51-69. Amsterdam, Oxford, New-York.

Thollard 1984 : THOLLARD (P.) - Strabon, Lyon, Vienne et les Ségusiaves. Revue archéologique de Narbonnaise, 17, 1984, p. 115-122.

Thiriot 1992 : THIRIOT (J.). - Céramiques fines islamiques du Midi de la France au Bas MoyenAge. Actes du IVe Colloque International : Cerâmica medieval do Mediterrâneo ocidental (Lisbonne 1987), Mertola 1992. p. 285-304

Vadet 1981: VADET (A.) - Mercin et Vaux (Aisne). Un habitat gallo-romain. A. Vadet: les ossements animaux. Cahier Archéologique de Picardie 1981, n 8, p. 115-147.

Van den Driesch et Boessneck 1974 : VAN DEN DRIESCH (A.) et BOESSNECK (J.) - Kritische Ammerkungen zur Widerristhöheberechnungen an Längenmassen von frühgeschichtlicher Tierknochen. Säugetierkdl. Mitt. 22. p. 325-348.

Verhaeghe 1991 : VERHAEGHE (Fr.) - L'espace civil et la ville in: Archéologie des villes dans le nord-ouest de l'Europe. Acte du 4ème Congrès International d'Archéo Méd., Douai, 1991.

Vicard 1984 : VICARD (T.) - Principes pour une évolution de la céramique régionale. Le XIIIe s. dans le département du Rhône. Mémoire de Maîtrise, dactylographié, Université Lyon 2, 1984.

Vicard 1985 : VICARD (T.) - Deux dépotoirs du Château d'Anse (Rhône) au XIIIe s., p.52-81. in : Etude Céramologique. Brochure polycopiée du Groupe de travail en céramologie, 1985.

Villedieu 1983 : VILLEDIEU (Fr.) - Avenue A. Max. Ligne D du Métropolitain de Lyon. Rapport D.A.H, 1983.

Villedieu 1990 : VILLEDIEU (F.) - Lyon, Saint-Jean. Les fouilles de l'avenue Adolphe Max. D.A.R.A., $\mathrm{n}^{\circ} 3,1990,239 \mathrm{p}$.

Walker 1981: WALKER (S.) - Récentes recherches en archéologie gallo romaine et paléochrétienne sur Lyon et sa région. Ed. B.A.R. International, Séries 108, Lyon, 1981, 54 p.

Wuilleumier 1953 : WUILLEUMIER (P.) - Lyon, métropole des Gaules. Ed. Les belles lettres, Paris, 1953, 114 p. 